\title{
Rehearsal-Strategien im Grundschulalter -
}

\section{Allgemeine und differentielle Entwicklungsveränderungen}

\author{
Dissertation \\ zur Erlangung des Doktorgrades \\ der Mathematisch-Naturwissenschaftlichen Fakultäten \\ der Georg-August-Universität zu Göttingen
}

vorgelegt von

Michael Lingen

aus Meppen

Göttingen 2003 


\section{7}

Referent: Prof. Dr. Marcus Hasselhorn

Korreferent: Prof. Dr. Roland Brünken

Tag der mündlichen Prüfung: 20. 01. 2004 
We ought to stand up and look the world frankly in the face.

We ought to make the best we can of the world, [...].

A good world needs knowledge, kindliness, and courage.

Bertrand Russel

Kein Mensch verfügt über die absolute Wahrheit.

Widerstand gilt daher denen, die ihre begrenzte

Überzeugung anderen aufzwingen wollen.

Richard von Weizsäcker 


\section{Vorwort}

Der Forschungsprozess, der zu dieser Arbeit geführt hat, gleicht einer Expedition in ein unbekanntes Land. Die Entdeckung dieses neuen Landes, der Memorierstrategie, war ein herausforderndes und bereicherndes Unterfangen. Oft war der Weg beschwerlich, der vor mir liegende Pfad ungewiss. Zum Glück hatte ich Gefährten, die mich auf dieser Reise begleitet haben.

Meinem Doktorvater und Mentor Prof. Dr. Marcus Hasselhorn danke ich für seinen fachlichen und persönlichen Rat und sein Vertrauen in mich und meine Arbeit. Sein Vorbild war für mich stets Wegweiser und Kompass auf der Reise. Maren Richter, meiner Wegbegleiterin und Kollegin, danke ich für viele Stunden der Planung, Gestaltung und Realisierung des gemeinsamen Weges. Wenn auch ihr Reiseziel ein anderes ist, so teilen wir doch die Sorgen und Freuden der gleichen Fahrt.

Ohne die Bereitschaft vieler Kinder zur Teilnahme an der entwicklungspsychologischen Studie und die Offenheit ihrer Eltern, die die Datenerhebung ermöglicht haben, wäre ein so großes Forschungsprojekt nicht realisierbar gewesen. Ihnen allen gebührt mein Dank. Die Erhebung und Auswertung der Daten war erst durch die engagierte und unermüdliche Arbeit vieler Hilfskräfte und ProjektmitarbeiterInnen möglich. Besonders hervorzuheben ist hier der Beitrag von Nadine Büttner, Vera Frühauf, Martin Lehmann, Anke Prasse, Patrick Theisen und Saskia Victor.

Mein Dank gilt allen Kolleginnen und Kollegen, die mich auf verschiedene Weise unterstützt und ermutigt haben. Insbesondere bedanke ich mich bei Prof. Dr. Roland Brünken, Jörg Behrendt, Stephanie Schreblowski und nochmals Martin Lehmann für die konstruktiven Vorschläge zur Gestaltung dieser Arbeit.

Nicht zuletzt danke ich Heike für ihren emotional support. Der Gedanke an sie hat mir auf meiner Reise Kraft und Zuversicht gespendet.

Michael Lingen

Göttingen, 10. 12. 2003 


\section{Inhaltsverzeichnis}

1. Einleitung.

2. Theoretischer und empirischer Hintergrund .........................................................

2.1 Phänomene strategischer Gedächtnisprozesse bei Kindern ........................................4

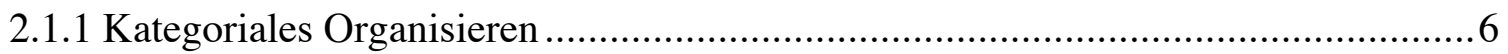

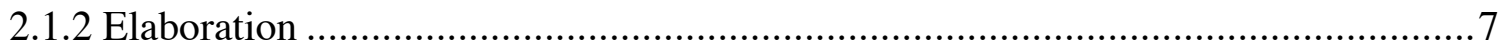

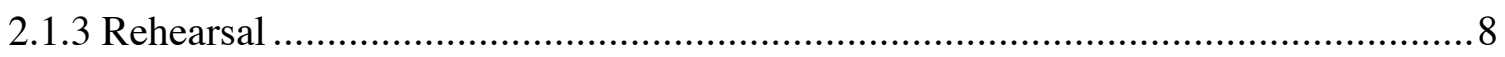

2.2 Entwicklungsmodelle strategischer Gedächtnisprozesse...................................... 19

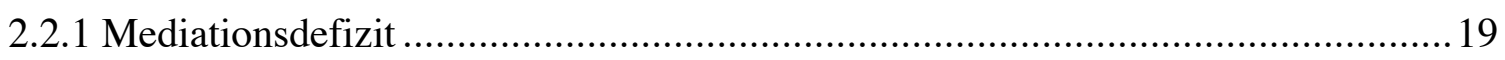

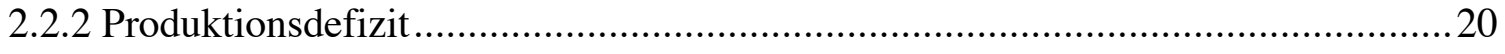

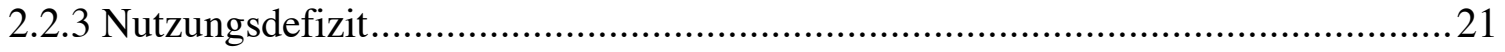

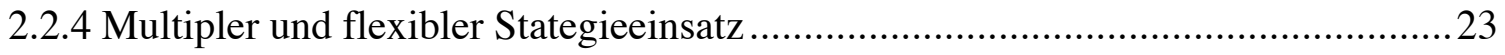

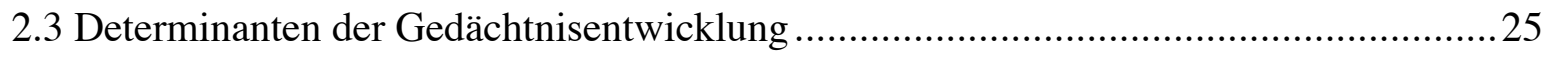

2.3.1 Das Kurzzeitgedächtnis und die Gedächtniskapazität .....................................26

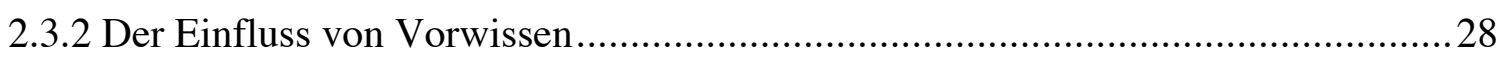

2.3.3 Die Bedeutung metakognitiver Prozesse ....................................................... 32

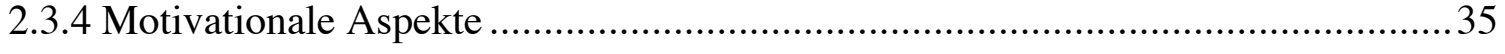

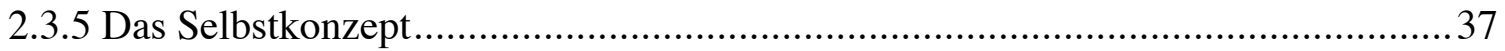

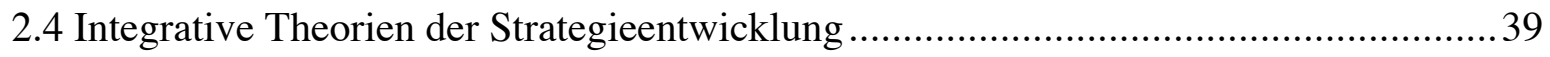

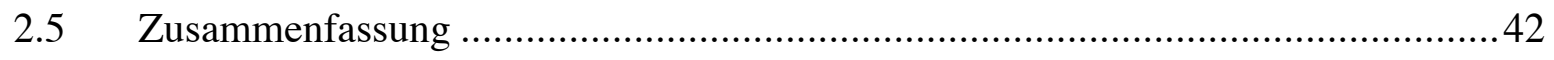

3. Eigene Untersuchung und psychologische Hypothesen ............................................ 44

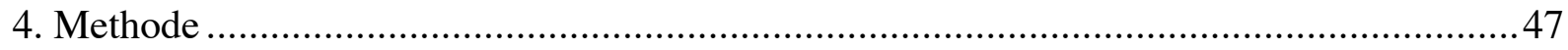

4.1 Untersuchungsdesign und Auswahl der Stichprobe ..................................... 47

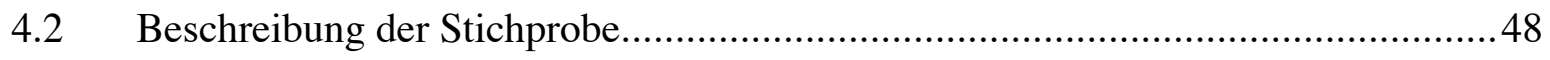

4.3 Verwendete Verfahren und abhängige Variablen ........................................... 49

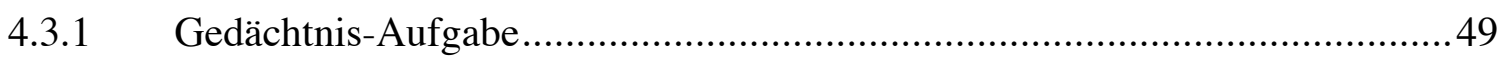

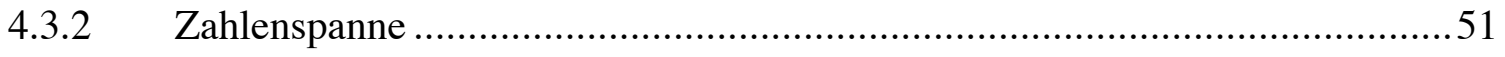

4.3.3 Metakognitives Strategiewissen............................................................ 51

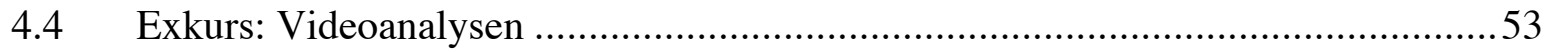

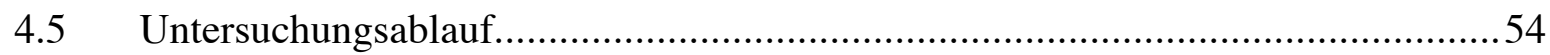




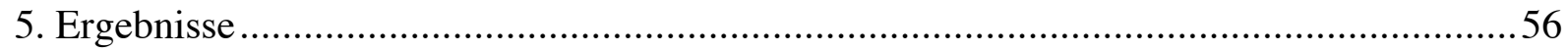

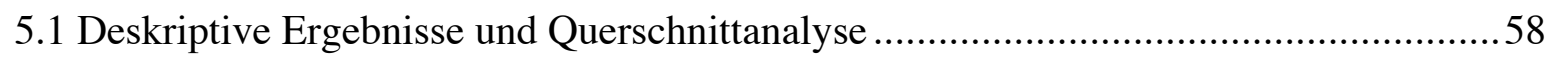

5.1.1 Psychologische Hypothese und Vorhersagen...................................................58

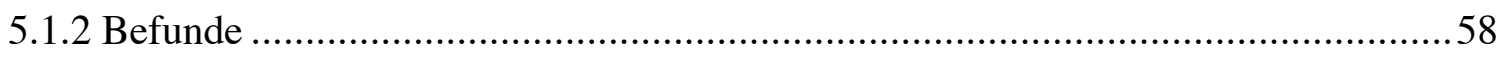

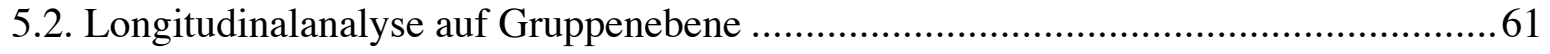

5.2.1 Psychologische Hypothesen und Vorhersagen....................................................61

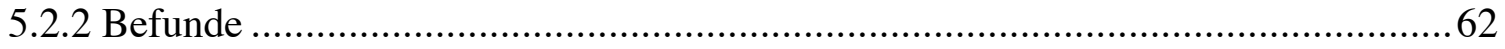

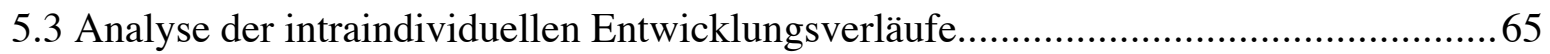

5.3.1 Psychologische Hypothesen und Vorhersagen................................................65

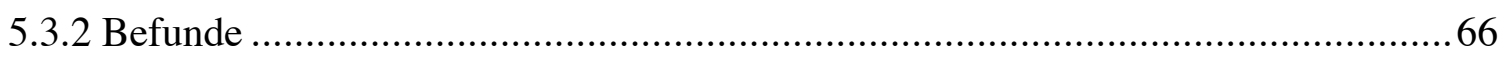

5.4 Die Bedeutung metakognitiver und kapazitiver Aspekte - Überprüfung der Vorhersagen

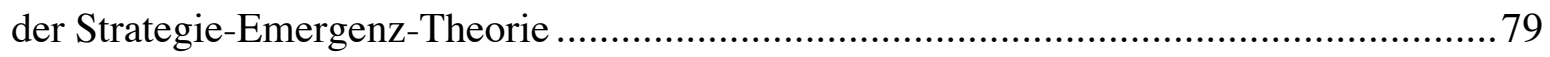

5.4.1 Psychologische Hypothesen und Vorhersagen................................................. 79

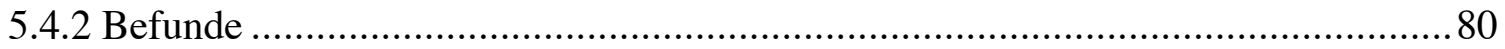

5.5 Belege für eine Nutzungsineffizienz bei kumulativem Rehearsal ............................ 88

5.5.1 Psychologische Hypothese und Vorhersagen.................................................. 88

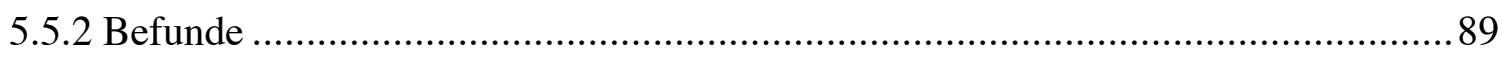

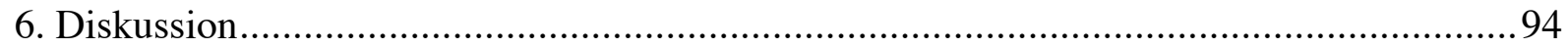

6.1 Zusammenfassung der empirischen Befunde........................................................94

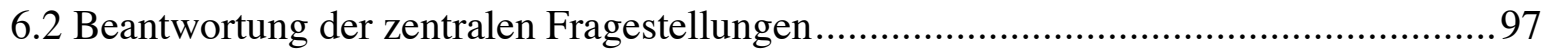

6.3 Einordnung der Ergebnisse in Befunde und Modelle der entwicklungspsychologischen

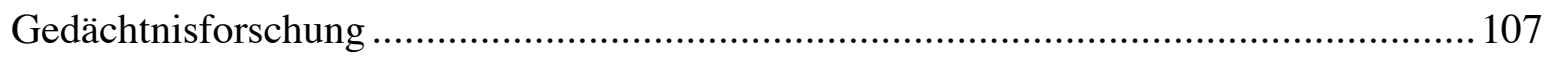

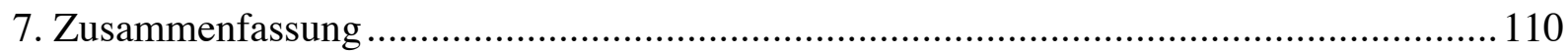

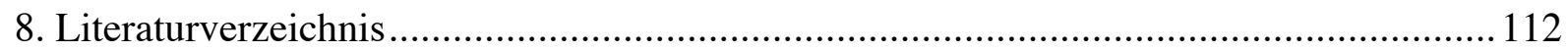

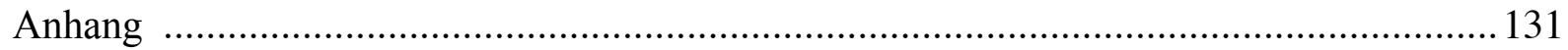




\section{Einleitung}

Die Entwicklung des Gedächtnisses und die damit verbundenen kognitiven Prozesse sind ein zentrales Thema der kognitiven Entwicklungspsychologie. Im Vordergrund stehen dabei die beiden Fragen, was sich entwickelt und warum die Entwicklung einen bestimmten Verlauf nimmt. Die erste Frage kann beantwortet werden, indem man die Veränderungen der kognitiven Leistungsfähigkeit von Kindern über die Zeit hinweg erfasst. Zur Beantwortung der zweiten Frage müssen die Befunde über die kognitive Entwicklung und ihre Begleitumstände zu einer Theorie der Entwicklung kognitiver Fertigkeiten und des Wissens integriert werden (vgl. Goswami, 2001). Die Veränderungen zu dokumentieren ist vergleichsweise einfach, sie zu erklären stellt jedoch nach wie vor eine Herausforderung dar (vgl. GoldinMeadow, 2001) und „requires us to develop causal explanations for observed cognitive changes" (Goswami, 1998).

In ihrem Beitrag über die historischen Trends der entwicklungspsychologischen Gedächtnisforschung verweisen Schneider und Pressley (1997) darauf, dass es seit 1880 drei Phasen mit zum Teil unterschiedlichen Forschungslinien gegeben hat (vgl. auch Schneider, 2000):

- Die meisten Studien zur Gedächtnisentwicklung aus dem späten 19. und frühen 20. Jahrhundert wurden in Europa durchgeführt und befassten sich mit der Gedächtnisspanne unter verschiedenen Bedingungen. Unter der Gedächtnisspanne versteht man dabei die Anzahl der Items (Zahlen, Wörter, Buchstaben), die unmittelbar seriell korrekt wiedergegeben werden kann. Obwohl sich die meist experimentellen Studien hauptsächlich mit dem Kurzzeitgedächtnis beschäftigten, wurden vereinzelt auch Studien zum Langzeitgedächtnis und zu Vergessensprozessen durchgeführt (z.B. Ebbinghaus, 1897). Die meisten Untersuchungen dieser Periode beschränkten sich jedoch auf die reine Beschreibung von Gedächtnisphänomenen und deren altersabhängigen Veränderungen, ohne kausale Zusammenhänge zu erfassen.

- In einer zweiten Phase, den so genannten „dry middle years“ (Ceci \& Bruck, 1993) von 1936 bis 1945, stockte in Europa der wissenschaftliche Fortschritt aufgrund des zweiten Weltkrieges. In Amerika wurden unter dem Einfluss des Behaviorismus Studien zu Lernparadigmen und zum Diskriminationslernen durchgeführt. In der Sowjetunion waren die Wissenschaftler dagegen besonders an der Entwicklung des Gedächtnisses für bedeutungshaltiges Lernmaterial interessiert. 
- Die Ära ab 1965 unterscheidet sich in zweierlei Hinsicht von den historischen Ansätzen: Als Erstes fokussiert das moderne Konzept von Gedächtnis stärker auf die den beobachtbaren Veränderungen zugrunde liegenden Mechanismen. Dadurch wird der funktionale Aspekt der Gedächtnisentwicklung betont (vgl. Hasselhorn \& Grube, im Druck). Die zweite Unterscheidung beruht auf den seit 1960 entwickelten Gedächtnismodellen, die einen großen Einfluss auf die Gedächtnisforschung hatten und haben. Aus dieser neuen Perspektive heraus kann Gedächtnisentwicklung sowohl die hardware (z.B. die Veränderungen der Gedächtniskapazität und der Verarbeitungsgeschwindigkeit) als auch die software (z.B. die Nutzung von Gedächtnisstrategien) umfassen.

Die moderne Ära der kognitiven Entwicklungspsychologie begann Mitte der sechziger Jahre mit der Erforschung mnemotechnischer Strategien, den so genannten Gedächtnisstrategien, und ihrer Rolle bei der Speicherung und dem Abruf von Informationen (Schneider, 2000). Unter Gedächtnisstrategien versteht man ,kognitive Aktivitäten oder Verhaltensweisen, die bewusst vom Subjekt kontrolliert und angewandt werden, um die Gedächtnisleistung zu steigern“ (Naus \& Ornstein, 1983, S. 12). Die Erforschung dieses besonderen Typs kognitiver Prozesse dominierte das Fach bis weit in die achtziger Jahre, und nach wie vor stellt das Strategiekonzept einen wichtigen Forschungsgegenstand dar (Schneider, 2000; Schneider \& Bjorklund, 1998). Die Gedächtnisstrategien befinden sich deswegen im Mittelpunkt zahlreicher Untersuchungen, da sich hier ausgeprägteste interindividuelle Unterschiede zeigen und sie eine zentrale Rolle bei der Gedächtnisentwicklung und der allgemeinen kognitiven Entwicklung spielen (vgl. Harnishfeger \& Bjorklund, 1990).

Die Mehrzahl der Studien zu Gedächtnisstrategien hat sich mit den Enkodierstrategien befasst, die bei der Einspeicherung von Informationen gebraucht werden und die dazu beitragen, neue Informationen (dauerhaft) zu behalten. Die am häufigsten untersuchten mnemotechnischen Strategien sind:

- Organisation (Gruppieren des Lernmaterials nach semantischen Kategorien),

- Elaboration (Einbettung des Lernmaterials in einen bedeutungsvollen Kontext) und

- Rehearsal (das Vor-sich-Hersagen der zu behaltenden Inhalte).

Das Forschungsinteresse an diesen Prozessen war stark durch die bedeutende Rolle bedingt, die ihnen in den Mehrspeichermodellen des Gedächtnisses zugesprochen wurde. Darüber hinaus hatten die Arbeiten von John Flavell (zusammengefasst bei Flavell, 1970) einen intensiven Einfluss auf die Erforschung des strategischen Gedächtnisverhaltens (vgl. Howe \& O'Sullivan, 1990). Neben den drei genannten gibt es noch weitere, z.T. allgemeinere Strate- 
gien (z.B. Chunking, Selection, Loci-Technik), die bislang jedoch kaum unter entwicklungspsychologischer Perspektive untersucht worden sind.

Im Zentrum dieser Arbeit steht die Entwicklung des sogenannten Rehearsals und ihre Bedeutung für die wachsende Gedächtnisleistung im Verlauf der späten Grundschuljahre. Als Rehearsal bezeichnet man allgemein einen Memorier- bzw. Wiederholungsprozess der zu lernenden Inhalte. Rehearsal-Strategien sind wenig effektiv für das langfristige Behalten, da der Lernende die Informationen nicht vollständig (d.h. semantisch) verarbeitet. Sie stellen jedoch in Verbindung mit anderen Gedächtnisstrategien, wie z.B. Organisationsprozessen und Elaborationen, eine sehr effektive Strategie dar. Diese benötigen Rehearsal-Prozesse, damit die Informationen lange genug im Kurzzeitgedächtnis verbleiben, um sie organisieren oder verbinden zu können, bzw. damit sie lange genug ihre Bedeutung behalten, um Informationen aus dem Langzeitgedächtnis abzurufen. Trotz einschlägiger Forschungsbemühungen wurden vergleichsweise wenige Studien zu den Memorier- oder Rehearsal-Strategien durchgeführt. Die vorwiegend auf Altersvergleichen basierenden Befunde wurden lediglich durch zwei kleinere Längsschnittstudien untermauert (Guttentag, Ornstein \& Siemens, 1987; Kunzinger, 1985). Diese sind in ihrer Aussagekraft hinsichtlich individueller Entwicklungsverläufe jedoch sehr eingeschränkt, da lediglich zwei Messzeitpunkte mit einem größeren Abstand zwischen den Testungen realisiert wurden.

Obwohl Rehearsal-Prozesse aufgrund ihrer zentralen Funktion für das Behalten als die prototypischen Gedächtnisstrategien gelten, werden sie seit den neunziger Jahren kaum noch thematisiert und können daher immer noch als ,ill-defined group of memory strategies“ (Flavell, 1985, S. 218) bezeichnet werden (vgl. Schneider \& Pressley, 1997). Die Zielsetzung der vorliegenden Arbeit ist es, die individuelle Entwicklung dieser speziellen Gedächtnisstrategien zu untersuchen.

Zur Herleitung der Fragestellungen der Arbeit aus dem theoretischen und empirischen Hintergrund werden in Kapitel 2 die Phänomene strategischer Gedächtnisprozesse bei Kindern dargestellt. Besonders ausführlich werden die Befunde zur Entwicklung von RehearsalProzessen erörtert. Im Anschluss daran werden Defizite, die bei der Ausbildung von Gedächtnisstrategien diskutiert werden, sowie Annahmen über eine flexible und multiple Strategieentwicklung und -wahl, jeweils mit dem Fokus auf Rehearsal, berichtet. Die Erörterung weiterer Determinanten der Gedächtnisentwicklung und integrativer Theorien der Strategieentwicklung bilden den Abschluss dieses Kapitels.

Die zentralen Fragen der vorliegenden Arbeit und die Explikation der zugrunde gelegten Hypothesen aus den vorab berichteten Befunden sind Inhalt des dritten Kapitels. In Kapi- 
tel 4 erfolgen die Darstellung des Untersuchungsdesigns und die Stichprobenbeschreibung. Daran schließen sich Angaben über die verwendeten Verfahren und Abhängigen Variablen sowie zum Untersuchungsablauf an.

Die im empirischen Teil der Arbeit berichteten Analysen und Befunde sind in fünf Abschnitte aufgeteilt. Nach einem querschnittlichen Klassenvergleich und längsschnittlichen Analysen auf Gruppenebene werden Befunde zur intraindividuellen Entwicklung von Rehearsal vorgestellt. Daran schließen sich die Prüfung der bewussten Nutzungsmöglichkeit (Emergenz) strategischen Verhaltens sowie kapazitiver Einflüsse und die Beantwortung der Frage nach der Leistungsdienlichkeit erster spontaner Rehearsal-Prozesse an. Im Schlussteil erfolgt eine Zusammenfassung der Befunde, ihre Diskussion im Hinblick auf die formulierten Fragestellungen sowie ihre Einordnung in Befunde und Modelle der entwicklungspsychologischen Gedächtnisforschung.

\section{Theoretischer und empirischer Hintergrund}

\subsection{Phänomene strategischer Gedächtnisprozesse bei Kindern}

Obgleich hinsichtlich der Terminologie zahlreiche Unterschiede bestehen und der Begriff Gedächtnisstrategien kontrovers diskutiert wird, können sich die meisten Wissenschaftler einer Definition anschließen, die sie als ,potentially conscious, deliberate, and controllable cognitive plans adopted to enhance performance in memory tasks" bezeichnet (Schneider \& Bjorklund, 1998, S. 475). Hasselhorn (1996) fand in der Literatur unterschiedliche Charakteristika strategischen Verhaltens: „Danach sind Strategien 1) intentionale, 2) zielgerichtete, 3) spontane, 4) bewusste, 5) unter der Kontrolle einer Person stehende, 6) kapazitätsbelastende und 7) selektive Prozesse, die 8) nicht lediglich automatisch bzw. obligatorische Konsequenzen der Aufgabenbearbeitung sind“ (S. 59). Gedächtnisstrategien können in der Lernbzw. Enkodierphase, bei der Speicherung von Lernmaterial und in der Abrufphase (Retrieval) angewandt werden. Viele alterskorrelierte Veränderungen bei Gedächtnisleistungen reflektieren die Aneignung neuer Strategien, die Ausdifferenzierung verfügbarer Strategien oder den Transfer einer gelernten Strategie auf eine neue Lernanforderung (vgl. Siegler, 2001).

Nach Waters und Andreassen (1983) gibt es, trotz einer enormen Variationsbreite zwischen den jeweiligen Strategien, Merkmale, die allgemein für den Erwerb mnemotechnischer Strategien charakteristisch sind:

- Anfangs wenden Kinder eine neue Gedächtnisstrategie nur auf wenige Situationen an. 
- Sie beschränken sich dabei auf einfaches, interessantes Lernmaterial und Situationen mit geringen kognitiven Anforderungen.

- Der Gebrauch einer neuen Strategie erfolgt noch relativ rigide, und die Kinder zeigen kaum einen Transfer auf veränderte Situationen oder Lernanforderungen.

Ornstein, Baker-Ward und Naus (1988) haben ein allgemeines Rahmenmodell vorgestellt, das die Entwicklung von Gedächtnisstrategien von ihrer ersten, vorläufigen Anwendung in spezifischen, möglicherweise supportiven Kontexten bis zur effizienten Strategienutzung bei einem breiten Spektrum von Anforderungen erklärt. Dieses informelle Modell postuliert ein Kontinuum mnemotechnischer Effektivität, auf dem zumindest fünf Stufen unterschieden werden können:

- In einem frühen Bereich dieses Kontinuums nutzen Kinder keine Strategien bei der Bearbeitung von Gedächtnisaufgaben.

- Etwas später, im Vorschulalter, zeigen Kinder in spezifischen Lernkontexten strategisches Verhalten. Die Erfolge dieser mnemotechnischen Bemühungen werden jedoch von den Kindern nicht als tatsächlicher Gewinn der Erinnerung wahrgenommen.

- Im Grundschulalter können die mnemotechnischen Bemühungen recht effizient sein; Entwicklung und Gebrauch von Strategien werden aber teilweise von der Art und Bedeutsamkeit der Stimuli bestimmt.

- Im weiteren Verlauf werden Strategien effizient in verschiedenen Situationen und bei unterschiedlichem Lernmaterial verwendet.

- Schließlich wird die Strategienutzung effizienter, was die Routine und Automatisierung widerspiegelt, die zum einen durch Übung und zum anderen durch die Entwicklung basaler Fähigkeiten der Informationsverarbeitung entstehen (z.B. die Fähigkeit, Verbindungen innerhalb des bereits vorhandenen Wissens herzustellen).

In der überwiegenden Mehrheit der seit Mitte der sechziger Jahre durchgeführten Studien wurde mit Kindern zwischen 6 und 12 Jahren gearbeitet. In diesem Zeitraum finden die größten Entwicklungsveränderungen beim Strategienerwerb statt. Verschiedene Befunde zeigten jedoch, dass Kinder bereits im Vorschulalter bis zu einem gewissen Grad mnemotechnische Strategien einsetzen. Experimentelle Untersuchungen von Sommerville, Wellman und Cultice (1983) erbrachten Belege dafür, dass bereits Zweijährige Gedächtnisstrategien einsetzen, um vorauszuplanen und ein bestimmtes Ereignis nicht zu vergessen. DeLoache, Cassidy und Brown (1985) verwendeten ein Versteckspiel zur Untersuchung von Gedächtnisstrategien bei Kindern zwischen 18 und 24 Monaten. Die Kinder nutzten in Abhängigkeit von der Anforderung strategisches Selbsterinnerungsverhalten, um sich zu merken, wo ein Spiel- 
zeug versteckt worden war. Auch wenn diese und ähnliche Studien Evidenz dafür erbringen, dass bereits Zwei- bis Vierjährige erstaunliche strategische Kompetenzen aufweisen, zeigen sie ebenfalls, wie fragil diese Gedächtnisaktivitäten bei Vorschulkindern sind und wie material- bzw. situationsspezifisch sie eingesetzt werden. Ältere Kinder nutzen dagegen ihre Fähigkeiten in unterschiedlichen Situationen und bei einer Vielfalt von Aufgabentypen. In den folgenden Abschnitten werden die drei am besten untersuchten Enkodierstrategien exemplarisch dargestellt. Da die Rehearsal-Strategien im Fokus der vorliegenden Untersuchung stehen, werden sie im Anschluss an das kategoriale Organisieren und die Elaboration ausführlicher behandelt.

\subsubsection{Kategoriales Organisieren}

Kategoriales Organisieren ist ein Prozess, bei dem verschiedene Elemente als zusammengehörig angesehen und nach Gruppen geordnet werden. Dies ist einer der fundamentalsten kognitiven Aktivitäten (Medin \& Aguilar, 1999). Der Vorgang des Kategorisierens unterstützt nahezu alle kognitiven Tätigkeiten, indem die Komplexität der eintreffenden Reize durch konzeptuelle Verarbeitung reduziert wird. Das erleichtert die Informationsaufnahme, die Verarbeitung und den Informationsabruf aus dem Gedächtnis. Die Organisation von Lernmaterial (Bilder, Wörter) in bedeutungsvolle Kategorien und der Einfluss dieser Strategie auf die Reproduktionsleistung gehört zu den am besten untersuchten Prozessen der kognitiven Entwicklungspsychologie (vgl. Bjorklund \& Douglas, 1997; Schneider \& Bjorklund, 1998). In der prototypischen Aufgabe hierzu werden den Kindern eine Reihe von kategorisierbaren Bildern oder Wörtern gleichzeitig für einen längeren Zeitraum vorgelegt, die sie möglichst gut behalten und in beliebiger Sequenz reproduzieren sollen (Sort Recall). Die Auswahl der Items erfolgt auf der Grundlage altersentsprechender Wortnormen (z.B. Hager \& Hasselhorn, 1994; Hasselhorn, Jaspers \& Hernando, 1990). Das Ausmaß an Organisationsverhalten während der Lernphase (Sortieren) und der Abrufphase (Clustern) wird unter Verwendung verschiedener Indizes erfasst (vgl. Bousfield, 1953; Bousfield \& Bousfield, 1966; Hasselhorn, 1996; Robbins \& Nolan, 2000). Die beiden gebräuchlichsten Maße sind der Ratio of Repetition (RR; Cohen, Sakoda \& Bousfield, 1954) und der Adjusted Ratio of Clustering (ARC; Gerjuoy \& Spitz, 1966; Roenker, Thompson \& Brown, 1971).

Die organisationalen mnemotechnischen Strategien entwickeln sich nach Ansicht der meisten Autoren erst am Ende der Grundschulzeit, also etwas später als erste RehearsalProzesse. Bis etwa zum neunten Lebensjahr ist die Lernleistung bei kategorisierbarem Lern- 
material ebenso hoch wie bei Itemlisten ohne kategoriale Zusammenhänge (Flavell \& Wellman, 1977). Darüber hinaus ist der frühe Einsatz dieser Strategie von der (motivierenden) Aufgabenstellung und dem Lernmaterial abhängig. Schneider (1986) stellte fest, dass nur 10 Prozent der Siebenjährigen in seiner Untersuchung Bilder mit hoher Inter-Item-Assoziativität spontan nach ihrer Kategorienzugehörigkeit ordneten. Bei den Zehnjährigen waren es dagegen schon 60 Prozent. Die Zehnjährigen setzten das Gruppieren der Items, unabhängig vom Ausmaß der Assoziativität zwischen den Items, gezielt und systematisch als Strategie ein und waren sich des Nutzens organisationaler Strategien für die Lernleistung bewusst. Der Einsatz von Organisationsstrategien tritt bei älteren Kindern also eher spontan sowie unabhängig von Lernmaterial und unterstützendem Kontext auf. Die Befunde bedeuten jedoch nicht, dass jüngere Kinder diese Strategie nur dann anwenden und dadurch bessere Lernleistungen erzielen können, wenn sie entsprechend instruiert werden (z.B. Bjorklund \& Coyle, 1995; Lange \& Pierce, 1992; Schneider \& Sodian, 1988). Jedoch können bereits kleine Veränderungen des Lernmaterials das (spontane) Sortier- und Clusterverhalten von Vorschulkindern massiv beeinflussen (vgl. Guttentag \& Lange, 1994). Die spontane und effektive (,strategische“) Nutzung von Organisationsprozessen, relativ unabhängig von Lernmaterial und Kontextbedingungen, kann meist erst ab dem Ende der Grundschulzeit beobachtet werden (vgl. Schneider \& Bjorklund, 1998). Generell gilt dabei, dass Kinder und Erwachsene die kategoriale Organisationsstrategie (erst) dann nutzen können, wenn sie ausreichend metakognitives Wissen über das Lernmaterial besitzen (vgl. Hasselhorn, 1996).

\subsubsection{Elaboration}

Eng verbunden mit dem kategorialen Organisieren ist die Elaboration. Diese Strategie besteht darin, dass bedeutungsvolle Verbindungen zwischen den zu behaltenden Items erstellt werden bzw., dass das Lernmaterial in einen semantischen Kontext eingebettet wird. Das kann z.B. dadurch erreicht werden, dass bildhafte oder sprachliche Assoziationen zu den Items oder zwischen Item-Paaren aufgebaut werden. Je tiefer oder elaborierter man die Bedeutung enkodiert, desto besser wird die Gedächtnisleistung (Craik \& Tulving, 1975). Eine Illustration dieser Annahme gibt eine Studie von Bradshaw und Anderson (1982). In ihrer Untersuchung mussten Testpersonen Fakten von Persönlichkeiten lernen (z.B. „An einem entscheidenden Punkt seines Lebens reiste Mozart von München nach Paris.“), wobei diese entweder mit oder ohne Zusatzinformationen dargeboten wurden (z.B. „Mozart wollte München verlassen, um einer verwickelten Liebesgeschichte zu entgehen.“). Die Probanden 
konnten mehr Fakten wiedergeben, zu denen sie Elaborationen erhalten hatten, als Fakten, die alleine dargeboten worden waren.

Der spontane Einsatz dieser komplexen Enkodierstrategie wird oft erst während der Adoleszenz beobachtet, obwohl ihre Anwendung bereits jüngeren Kindern durch Training vermittelt werden kann (Pressley, 1982; Siaw \& Kee, 1987). Einen Transfer findet man allerdings meist erst bei Sechzehn- bis Neunzehnjährigen. Selbst viele Erwachsene wenden diese Strategie nicht spontan an und müssen zu ihrer Verwendung aufgefordert werden (Rohwer, 1980).

Die meisten Studien, die den Einfluss von bildlicher oder verbaler Elaboration auf die Lernleistung untersuchen, verwenden Varianten der Methode des Paarassoziationslernens. Dabei werden als Lernmaterial Paare von unverbundenen Substantiven oder Objekten (z.B. Katze - Apfel, Bogen - Glas) benutzt. In der Testphase wird nur eines der jeweiligen Items, der Stimulus, vorgelegt, und die Probanden sollen das dazugehörige Item nennen. Die Befunde solcher Studien sprechen dafür, dass durch den Prozess der Elaboration ein Abrufpfad bzw. eine Verknüpfung zwischen Ursache (Stimulus) und Folge (Response) aufgebaut wird. In der Testphase kann die Zielinformation dann entweder direkt oder indirekt, über die Verknüpfung, abgerufen werden (vgl. Pressley \& Schneider, 1997; Schneider \& Pressley, 1997).

\subsubsection{Rehearsal}

Unter dem Begriff Rehearsal werden in der Gedächtnisforschung eine Reihe unterschiedlicher Gedächtnisstrategien zusammengefasst (Weinert \& Schneider, 1996). Erste Anzeichen für inneres Wiederholen sind einfache Objektbenennungen (Labeling), gefolgt von Ein-Wort-Wiederholungen (passive rehearsal) und aktiven, größere Informationsmengen umfassenden Memorierprozessen (cumulative rehearsal). Im Verlauf der Entwicklung kann dabei ein Wechsel von relativ passiven zu aktiveren Rehearsal-Prozessen beobachtet werden, der mit einer Verbesserung der Behaltensleistung verbunden ist (vgl. Ornstein \& Naus, 1985).

Flavell, Beach und Chinsky publizierten 1966 eine Untersuchung, die die Nutzung von Rehearsal bei Kindern zwischen 5 und 10 Jahren beschreibt. In dieser klassischen Studie wurden Kindergartenkindern, Zweit- und Fünftklässlern sieben Bilder mit vertrauten Objekten gezeigt, von denen sie sich zwei bis fünf merken sollten. Während der Lernphase trugen die Kinder einen Helm, dessen Visier ihre Augen verdeckte, den Mund jedoch frei ließ. Der Versuchsleiter zeigte nun sukzessiv auf einige der Bilder. Unmittelbar nach der Behaltensperiode bzw. mit 15 Sekunden Verzögerung wurden die gleichen Bilder in einer anderen Anordnung 
präsentiert. Die Kinder sollten in derselben Reihenfolge wie dies zuvor der Versuchsleiter getan hatte auf bestimmte Bilder zeigen (Serial Recall). Nur 10 Prozent der Fünfjährigen zeigten Lippenbewegungen oder wiederholten laut die Bilder, die sie später erinnern sollten. Bei den Siebenjährigen waren es dagegen 60 Prozent und bei den Zehnjährigen sogar 85 Prozent. Weiterhin erwies sich, dass mehr Bilder erinnert werden konnten, wenn die Kinder die Bilder häufiger memorierten. Flavell et al. (1966) schlussfolgerten daraus einen Zusammenhang zwischen der Häufigkeit von Rehearsal (erfasst über beobachtete Lippenbewegungen) und der Behaltensleistung. Genauere Analysen von Ornstein und Naus $(1978,1985)$ konnten jedoch belegen, dass Rehearsal kein einheitlicher Prozess ist und dass das spontane Rehearsal Siebenjähriger eher bruchstückhaft ausfällt und somit wenig behaltensfördernd ist. Die von Flavell et al. (1966) eingesetzte Technik des Lippenlesens lässt weiterhin keine tiefergehende Analyse des Rehearsal-Typs zu. Die Befunde hinsichtlich des allgemeinen Nutzens von Rehearsal für die Merkleistung wurden später unter anderem von Belmont und Butterfield (1971) und Liberty und Ornstein (1973) repliziert.

Die Studie von Flavell et al. (1966) war der Beginn für die Erforschung der Rolle der Gedächtnisstrategien, speziell der Rehearsal-Strategien, bei der Gedächtnisentwicklung. Das Interesse an den so genannten Wiederhol- bzw. Memorierstrategien wurde darüber hinaus auch durch ihre entscheidende Funktion im Multispeichermodell von Atkinson und Shiffrin (1968), im Ansatz der Verarbeitungstiefe von Craik und Lockhart (1972) und in dem Arbeitsgedächtnis-Modell von Baddeley (1986; Baddeley \& Hitch, 1974) geweckt (vgl. Schneider \& Bjorklund, 1998). Bereits sehr früh wurde von Flavell (1970) die Notwendigkeit betont, Forschungsmethoden zu finden, die eine direkte Beobachtung dieser strategischen Prozesse ermöglichen.

Methodische Aspekte: Bei den methodischen Zugängen zur Erfassung von RehearsalStrategien kann zwischen zwei Klassen von Vorgehensweisen unterschieden werden: Eine Gruppe von Techniken erlaubt nur indirekte Schlussfolgerungen über die Rehearsal-Aktivität, die andere beinhaltet die direkte Erfassung der Memorierprozesse (vgl. Ornstein \& Naus, 1978). Indirekte Verfahren sind die Beobachtung der Lippenbewegungen (z.B. Flavell, 1970), der Rückschluss auf Rehearsal-Prozesse aus den Positionskurven der freien Reproduktion (z.B. Hagen \& Kingsley, 1968), die Ableitung elektromyographischer Korrelate subvokaler Sprachprozesse während der Lernphase (Locke \& Fehr, 1970) und die Verwendung des von Belmont und Butterfield $(1969,1971)$ entwickelten Pause-Time-Paradigmas. Bei letzterem konnten die Probanden die verfügbare Lernzeit zwischen zwei Items selber bestimmen. Aus 
Veränderungen der gewählten Pausen zwischen zwei Itempräsentationen wurde hier indirekt auf die Rehearsal-Tätigkeit geschlossen.

Demgegenüber ermöglicht die Verwendung der Overt-Rehearsal-Technik (Rundus, 1971; Rundus \& Atkinson, 1970) eine direkte Beobachtung und genauere, quantitative und inhaltliche Analysen des verwendeten Rehearsal-Typs. Kennzeichnend für diese Methode ist das laute Memorieren des Lernmaterials. Eine weitere Informationsquelle ergibt sich aus den retrospektiven Selbstberichten über die Strategienutzung. Verschiedene Studien konnten zeigen, dass diese eng mit den beobachteten Strategien korrespondieren (z.B. Bray, Huffman \& Fletcher, 1999; McGilly \& Siegler, 1990). Gerade bei jüngeren Kindern sind die Selbstberichte jedoch teilweise unvollständig, sobald mehrere Strategien zur Anwendung kommen. Weiterhin erfordert die Information über die genutzte Strategie metastrategische Bewusstheit (Kuhn, 2000), die bei jüngeren Kindern oft noch nicht gegeben ist (s.u. Abschnitt 2.3.3). Schließlich kann das Wissen über die verwendete(n) Lernstrategie(n) nach dem Lerndurchgang sehr schnell verblassen.

In den frühen Arbeiten wurden hauptsächlich indirekte Maße als Beleg für eine Zunahme von Rehearsal-Prozessen interpretiert: Aus den so genannten seriellen Positionskurven wurde z.B. indirekt auf spontane Memorierprozesse geschlossen, wenn man besonders hohe Reproduktionsraten für zuerst dargebotene Items (Primacy-Effekt) und Items aus der Mitte der Lernliste fand (Belmont \& Butterfield, 1977; Hagen \& Stanovich, 1977). Die in diesem Bereich federführende Arbeitsgruppe um Ornstein und Naus hat dagegen hauptsächlich die Overt-Rehearsal-Technik zur direkteren Beobachtung von Rehearsal-Prozessen und deren Entwicklung eingesetzt.

Allerdings ist auch diese Methode nicht ganz unumstritten. In Studien von Kellas, McCauley und McFarland (1975b) sowie von Murray (1967) zeigten Erwachsene bei dieser Prozedur eine verringerte Abrufleistung im Gegensatz zur Reproduktion bei verdeckter Memoriertätigkeit (Covert-Rehearsal). Sanders, Murphy, Schmitt und Walsh (1980) konnten dagegen keinen negativen Einfluss der Overt-Rehearsal-Technik auf die Lernleistung feststellen. Während Rundus und Atkinson (1970) der Ansicht sind, dass das Overt-Rehearsal lediglich minimale Restriktionen beinhaltet, kommen Kellas et al. (1975b) zu folgendem Urteil: „The disparity between overt und covert study-times curves for free recall, in conjunction with the absence of correct response differences, suggests that the constraints placed on rehearsal by overt verbalization of stimuli are not what could be described as minimal.“ (S. 88, Hervorhebung im Original). Das „laute Denken“ führt nach Ansicht dieser Autoren zu starken Einschränkungen bei der Wahl der Verarbeitungsstrategie. 
Für Kinder kommen Ornstein, Naus und Liberty (1975) und Naus, Ornstein und Aivano (1977) zu ähnlichen Ergebnissen. Die Dritt- und Fünftklässler in der Studie von Kellas, McCauley und McFarland (1975a) zeigten jedoch keinen negativen Einfluss der OvertRehearsal-Technik auf ihre Memorier- und Lernaktivität, während die Siebtklässler eine Veränderung durch das erzwungene Lernverhalten verzeichneten. Oyen und Bebko (1996) kritisieren weiterhin, dass die Beschränkung auf die Overt-Rehearsal-Technik zur Unterschätzung der tatsächlichen Häufigkeit und Qualität von Rehearsal führen kann, da die Probanden möglicherweise nicht instruktionsgemäß alles laut aussprechen. Nach McGilly und Siegler (1989) ermöglicht die Kombination von unmittelbaren retrospektiven Selbstberichten über die Strategienutzung in Kombination mit Trial-by-Trial Beobachtungen des gezeigten Verhaltens mittels Overt-Rehearsal-Technik eine genaue Einschätzung der Strategienutzung in den einzelnen Lerndurchgängen. Angesichts der heterogenen Befunde zum „Lauten Denken“ und der offensichtlichen Nachteile eines einzelnen Maßes plädieren auch Oyen und Bebko (1996) für die Kombination zweier separater Methoden: die Beobachtung offener Rehearsal-Tätigkeit durch die Overt-Rehearsal-Technik und die getrennte Erhebung von weiteren Indikatoren, wie z.B. die Erfassung rhythmischer Kopf- bzw. Körperbewegungen (vgl. auch Goldin-Meadow, 2001) oder den retrospektiven Bericht über die Strategienutzung in der Nachbefragung.

Querschnittliche Befunde: Garrity (1975) konnte unter Verwendung elektromyographischer Methoden belegen, dass bereits vier- und fünfjährige Kinder subvokale Sprachprozesse bei verbalen Gedächtnisaufgaben zeigen. Der Umfang von Subvokalisation ist dabei alterskorreliert und steht in einem signifikanten Zusammenhang zur Lernleistung. Die von Kindergartenkindern und Erstklässlern gezeigten basalen Sprachprozesse in Lernaufgaben beschränken sich jedoch meist auf das einmalige Benennen (Labeling oder Naming) des jeweils neu präsentierten Wortes oder Objektes. Hagen (Hagen \& Kingsley, 1968; Hagen, Meacham \& Mesibov, 1970) erweiterte die Befunde zur Wirkungsweise des Labelings. Er wies nach, dass die bloße Benennung lediglich die Reproduktionswahrscheinlichkeit der zuletzt präsentieren Items erhöht, jedoch bei älteren Kindern mit dem Abruf früherer Listenitems interferiert. Letzteres wird als Beleg dafür gedeutet, dass bewusst eingesetztes Labeling die spontanen und aktiveren Rehearsal-Prozesse älterer Kinder stört (vgl. auch Hitch, Halliday, Schaafstal \& Heffernan, 1991). Nach Hagen und Kingsley (1968) gibt es - entsprechend der Mediationsdefizithypothese (Reese, 1962; s.u., Abschnitt 2.2.1) - einen bestimmten Altersbereich, in dem Labeling die Abrufleistung verbessert. In der Studie von Hagen und Kingsley (1968) trat dieser Effekt erst ab dem sechsten Lebensjahr auf, während jüngere Kinder keinen Gewinn aus den Labeling-Prozessen verzeichneten. Der fehlende Interferenzeffekt von Labe- 
ling auf die Erinnerungsleistung von Anfangsitems in dieser Altersgruppe legt nahe, dass die basalen Rehearsal-Prozesse bei Vorschulkindern Labeling-basiert sind (vgl. Ornstein \& Naus, 1978). Appel et al. (1972) vermerken, dass Labeling bei den jüngeren Kindern möglicherweise nur eine automatische, verbale Erkennungsreaktion und keine bewusste Gedächtnisstrategie ist. Danach wäre Labeling hier nur ein Zeichen dafür, dass ein jüngeres Kind das Stimulusmaterial identifiziert hat. Es ist damit jedoch noch nicht notwendigerweise bestrebt, sich die Items einzuprägen.

Während Vorschulkinder noch keine Unterscheidung zwischen Wahrnehmung (Perceiving) und Lernen (Memorizing) von Objekten zeigen, haben Erstklässler bereits ein Bewusstsein dafür, dass Lernen den Einsatz von planvollen und zukunftsorientierten kognitiven Aktivitäten erfordert (Appel et al., 1972). Allerdings wissen sie noch wenig darüber, was hinsichtlich mnemotechnischer Bemühungen und Aktivitäten zu tun ist, wenn sie mit einer speziellen Lernanforderung konfrontiert werden. Dementsprechend befinden sie sich in einer Art Übergangsphase. Kinder des zweiten und dritten Schuljahres nutzen dagegen mehrheitlich die Strategie des singulären Rehearsals, auch passives Rehearsal oder rote repetition genannt. Dabei wird ein Item während des freien Intervalls zwischen seriellen Itemdarbietungen mehrfach wiederholt. Henry und Millar (1993) vermuten, dass sich diese ersten basalen RehearsalProzesse aus dem zuvor geschilderten Benennungsverhalten entwickeln. Wenn die Kinder in die Schule kommen, werden sie vermehrt dazu aufgefordert, ihre optischen und taktilen Eindrücke verbal mitzuteilen. Nach Henry und Millar (1993) sind die Kinder somit zunehmend auf verbale Strategien zum Erlernen und Behalten von Informationen angewiesen. Eine zunehmende Geschwindigkeit und Leichtigkeit beim Benennen von Objekten führt letztlich zur Entdeckung von Rehearsal als Gedächtnisstrategie.

Den qualitativen Wechsel von passiven zu aktiveren Memoriertechniken verdeutlicht eine Untersuchung von Ornstein et al. (1975). Vorläuferstudien hatten Belege für eine altersmäßig ansteigende Behaltensleistung von Anfangsitems einer Liste mit nicht kategorisierbarem Lernmaterial und bessere Gesamtreproduktionsleistungen älterer Kinder erbracht. Diese besseren Leistungen ließen sich jedoch nicht alleine durch die Wiederholungshäufigkeit einzelner Items erklären. Ornstein et al. (1975, Experiment 1) führten eine detaillierte Studie über die qualitativen Unterschiede der Rehearsal-Prozesse bei Dritt-, Sechst- und Achtklässlern durch. Lernmaterial waren semantisch unverbundene Wörter, die visuell in einer seriellen Abfolge dargeboten wurden. Die Zeit zwischen zwei Items (Interstimulusintervall) betrug fünf Sekunden. Die eine Hälfte der Kinder wurde aufgefordert, das zuletzt gezeigte Wort und, wenn sie wollten, früher präsentierte Worte laut zu wiederholen. Die andere Hälfte erhielt 
keine spezielle Lerninstruktion. Der Abruf der Worte konnte in beliebiger Reihenfolge geschehen (Free Recall, vgl. Bower, 2000, S. 16). Es zeigten sich keine Altersunterschiede bei der Rehearsal-Häufigkeit der Items. Es gab jedoch ausgeprägte Unterschiede im RehearsalStil: Die Drittklässler tendierten dazu, das jeweils zuletzt präsentierte Wort alleine oder in Kombination mit wenigen weiteren Worten zu memorieren (zusammen etwa 2.5 Items). Die Sechst- und Achtklässler dagegen nahmen mehrere Worte (4.0 - 4.5) in ein Reherasal-Set auf (kumulatives Rehearsal). Diese Rehearsal-Sets bildeten somit eine Art funktionale Wiederholungseinheit. Ornstein et al. (1975) schlossen daraus, dass die entscheidenden Entwicklungsveränderungen im Rehearsal-Typ und nicht in der Rehearsal-Häufigkeit liegen.

In einer Folgestudie untersuchten Ornstein, Naus und Stone (1977) die Form des Zusammenhangs zwischen der altersgebundenen Veränderung der Rehearsal-Aktivität und den damit korrespondierenden Leistungsverbesserungen genauer. Dabei wurden Zweit- und Sechstklässler in verschiedenen Rehearsal-Techniken trainiert. Entsprechend früherer Befunde zeigten sich ohne Training deutliche Altersunterschiede in der Anzahl der zusammen memorierten Items. Diese Unterschiede im Rehearsal-Stil waren korrelativ verbunden mit Veränderungen in der Anzahl reproduzierter Anfangsitems. Die Lernleistung der untrainierten Zweitklässler war ebenso groß wie die der Zweitklässler, die jeweils ein bis zwei Items zusammen memorieren sollten. Die serielle Positionskurve dieser Gruppen zeigte keinen Primacy-Effekt. Dagegen waren die Lernleistungen und die Lernkurven der Zweitklässler, die kumulativ memorieren sollten, vergleichbar mit denen von untrainierten Sechstklässlern. Diese Befunde können als Evidenz dafür angesehen werden, dass es einen kausalen Zusammenhang zwischen der Rehearsal-Technik und der Lernleistung gibt. Weiterhin verdeutlichen sie, dass jüngeren Kindern die Nutzung aktiverer Memorierstrategien vermittelt werden kann, auch wenn sie diese nicht von selbst bzw. spontan generieren (s.u., Abschnitt. 2.2.2).

Weitere Hinweise für qualitative Unterschiede in den Rehearsal-Prozessen von Fünftklässlern, Achtklässlern und Erwachsenen ergeben sich aus der Studie von Cuvo (1975). Danach neigen Erwachsene stärker dazu, Worte vom Anfang einer seriell präsentierten Lernliste wieder in spätere Rehearsal-Sets einzubeziehen. Dies führt nach Cuvo (1975) zum einen zu einer längeren Verweildauer eines Items im Kurzzeitspeicher und beinhaltet zum anderen die Möglichkeit von Interitemassoziationen, da ein Wort im Verlauf mehrerer RehearsalDurchläufe mit verschiedenen Items in unmittelbare Kontiguität treten kann. Ähnliche Ergebnisse konnten Naus et al. (1977) mit Dritt- und Sechstklässlern erzielen. Die Sechstklässler nannten neben dem zuletzt präsentierten Wort zwei beliebige Worte aus dem Pool früher dargebotener Items und variierten ihre Rehearsal-Sets dadurch. Die Drittklässler kombinierten 
ein präsentiertes Wort immer mit den gleichen zwei anderen Items aus der Liste. In der Studie von Cuvo (1975) war die Anzahl an wiedereingelesenen Worten mit der Anzahl unterschiedlicher Worte in einem Rehearsal-Set korreliert. Nach Cuvo (1975) wird der entwicklungsmäBige Leistungszuwachs in Free-Recall-Aufgaben durch die Speichergröße (Buffer Size), den Rehearsal-Typ und die Anzahl an Rehearsal-Sets, in die ein Item eingebaut wird, vermittelt. Ein zusätzlicher Mechanismus des kumulativen Rehearsals ist eine Art Selbsttest durch die wiederholte Nennung der präsentierten Items. Somit ermöglicht die aktive Memorierstrategie Erfahrung mit der Erinnerung an frühere Stimuli vor der eigentlichen Abrufphase (Naus \& Ornstein, 1983; Ornstein \& Naus, 1978).

Durch den häufigen Gebrauch einer Strategie findet im Laufe der Zeit ein Automatisierungsprozess statt, der zu einer Verringerung der mentalen Anstrengungen bei ihrer Anwendung führt (Footo, Guttentag \& Ornstein, 1988). Dies erlaubt es Kindern, die Strategie auf neue Inhalte mit größeren Anforderungen zu übertragen (vgl. Siegler, 2001). Guttentag (1984) prüfte den Zusammenhang zwischen mentaler Anstrengung bei der Ausführung kumulativen Rehearsals und spontaner Nutzung dieser Strategie bei Kindern. Er führte mehrere Studien mit Dual-Task-Aufgaben durch. Neben einer Lernaufgabe sollten die Kinder verschiedenen Alters simultan eine einfache motorische Aufgabe (möglichst schnelles Drücken einer Taste) ausführen. Als Maß der mentalen Anstrengung diente die Verlangsamung der motorischen Aufgabe aufgrund des kumulativen Rehearsals. Die jüngeren Kinder zeigten eine solche Reduktion der Geschwindigkeit in der motorischen Leistung, die jedoch bei einfacher, passiver Wiederholung der Items ausblieb. Weiterhin war das Ausmaß an Interferenz der beste Prädiktor für die spontane Anwendung der Rehearsal-Strategie. Danach zeigten vor allem die Kinder, die die Rehearsal-Strategie wenig kognitive Ressourcen kostete, eine spontane Rehearsal-Aktivität und größere Rehearsal-Sets.

Längsschnittliche Befunde: Eine der wenigen Längsschnittstudien zur Entwicklung von Rehearsal-Strategien wurde von Kunzinger (1985) durchgeführt. Er erhob das Lernverhalten und die Lernleistung von Siebenjährigen bei einer Overt-Rehearsal-Aufgabe. Zwei Jahre später wurden 18 der ursprünglich 24 Kinder unter den gleichen Bedingungen nochmals getestet. Es resultierte ein genereller Anstieg der mittleren Rehearsal-Set-Größe von 1.7 auf 2.6. Die Rehearsal-Set-Größe war beim ersten Messzeitpunkt weitgehend unabhängig von der Abrufleistung, zeigte jedoch eine interindividuelle Stabilität über die Zeit $(r=.80)$ und konnte spätere Leistungsunterschiede der Neunjährigen vorhersagen. Nach Kunzinger (1985) spiegelt die Varianz in der Rehearsal-Set-Größe beim ersten Messzeitpunkt die sich entwickelnde aktivere Rehearsal-Strategie wider. Beim zweiten Messzeitpunkt gab es einen engen positiven $\mathrm{Zu}$ - 
sammenhang zwischen Rehearsal-Set-Größe und Gesamtreproduktion und einen negativen Zusammenhang zwischen der Reproduktion später dargebotener Listenitems und der Größe der Rehearsal-Sets. Dies ist ein Zeichen für eine Interferenz zwischen den beiden Faktoren. Je größer und durchmischter das Rehearsal-Set ist, desto eher kommen frühe Items in ihm vor, die mit späteren Items konkurrieren, deren Verarbeitung erschweren und ihre Reproduktionswahrscheinlichkeit verringern (vgl. auch Kunzinger \& Witryol, 1984). Insgesamt stützt die Studie von Kunzinger (1985) die Hypothese von Ornstein (Ornstein et al., 1975; Ornstein, Naus \& Stone, 1977), dass die Lernleistung durch die Größe und Qualität der Rehearsal-Sets determiniert wird und nicht durch die Rehearsal-Frequenz bzw. -Häufigkeit.

Guttentag, Ornstein und Siemens (1987) führten eine weitere Längsschnittstudie durch, bei der sie in Experiment 1 die spontane Anwendung von Rehearsal-Prozessen bei Kindern der dritten, vierten und sechsten Klassenstufe unter zwei Bedingungen der Itempräsentation (Standard- vs. Verfügbarkeitsbedingung) prüften. Zunächst konnten sich die Kinder durch die Präsentation von zwei Übungslisten mit sechs unverbundenen Wörtern mit der Aufgabe und der Overt-Rehearsal-Technik vertraut machen. Anschließend wurden drei Lernlisten mit jeweils 14 nicht-kategorisierbaren Wörtern (einsilbige Substantive) zum Lernen vorgegeben. Jedes Item wurde dem Kind per Tonband vorgesprochen und auf einem mit dem Item bedruckten Kärtchen für 5 oder 10 Sekunden präsentiert. Bei der Standardversion wurden die Wortkarten nach der Präsentation wieder verdeckt, in der unterstützenden Verfügbarkeitsbedingung blieben alle Karten bis zur Beendigung der Lernphase sichtbar liegen. Es war den Kindern dabei freigestellt, die Items in beliebiger Reihenfolge während der Interstimulusintervalle laut hörbar zu wiederholen. Mittels eines Tonbandes wurden die Rehearsal- und Recall-Aktivitäten aufgezeichnet. Die Ergebnisse dokumentieren unabhängig von der Klassenstufe aktivere Memorierprozesse unter der Verfügbarkeitsbedingung. Allerdings konnten nur die Sechstklässler einen weiteren Gewinn aus der zusätzlichen Zeit in der Zehn-SekundenBedingung ziehen. Dieses Ergebnis bestätigt die bereits von Ornstein und Naus (1985) geäuBerte Annahme, dass unter der Verfügbarkeitsbedingung die Informationsverarbeitungsanforderungen reduziert sind, da die Kinder die zuvor präsentierten Items einfach durch das Anschauen der vor ihnen liegenden Karten abrufen können. Zur genaueren Analyse der individuellen Rehearsal-Prozesse und Leistungen wurden die Kinder entsprechend ihres Rehearsal-Typs klassifiziert:

- Eine mittlere Rehearsal-Set-Größe von weniger als zwei Items unter beiden Bedingungen wurde als Single-Word-Rehearsal klassifiziert. 
- Als Übergangsphase (transitional) wurde ein Single-Word-Rehearsal unter der Standard-Bedingung in Kombination mit einer mittleren Rehearsal-Set-Größe von größer/gleich zwei Items unter der Verfügbarkeitsbedingung eingestuft.

- Kinder, die unter beiden Bedingungen eine durchschnittliche Rehearsal-Set-Größe von mehr als zwei Items hatten, wurden der Kategorie „cumulative, multi-item rehearsal“ zugeordnet.

Übereinstimmend mit Altersvergleichen aus früheren Studien fand sich ein alterskorrelierter Anstieg der Anzahl von Kindern mit kumulativem Memorierverhalten. Lediglich 13 Prozent der Drittklässler, aber bereits 71 Prozent der Viert- und sogar 92 Prozent der Sechstklässler konnten als kumulative Rehearser klassifiziert werden. Hinsichtlich der RehearsalSet-Größen ergaben sich jedoch keine Altersdifferenzen. Die Größe der Rehearsal-Sets unter der Verfügbarkeitsbedingung bei den als transitional klassifizierten Strategen unterschied sich nicht von denen der kumulativen Rehearser unter beiden Bedingungen. Die kumulativen Rehearser zeigten eine bedeutsam höhere Reproduktionsleistung als die Kinder der Transitional- und der Single-Word-Gruppen, deren Lernleistungen sich nicht signifikant voneinander unterschieden. Allerdings reproduzierten die kumulativen Rehearser der sechsten Klassenstufe signifikant mehr Items als Dritt- und Viertklässler mit gleichem strategischem Verhalten. Diese größere Effizienz der Strategienutzung wird nach Ansicht der Autoren durch unterschiedliche Qualitäten im Lernverhalten verursacht: Die älteren Kinder sahen beim Wiederholen oft nicht auf die visuell verfügbaren Items und führten auf diese Weise eine Art Selbsttest (self-testing) durch. Nach der Ansicht von Ornstein et al. (1987, S. 314) spiegelt dies Verhalten „,...children's developing conceptualization of the mechanisms by which memory strategies produce their effects" wider (Hervorhebung im Original). Es handelt sich also um eine Art metastrategisches Wissen (Kuhn, 2000; s.u., Abschnitt 2.3.3) darüber, warum mnemotechnische Strategien wie das Rehearsal effektiv sind.

Im Experiment 2 der Studie von Guttentag et al. (1987) wurde analog zu der Studie von Ornstein et al. (1975, s.u., Abschnitt 2.3.2) untersucht, ob die geordnete Darbietung von semantisch kategorisierbarem Lernmaterial eine verstärkte Anwendung von kumulativem Rehearsal hervorruft. Dafür bekamen Drittklässler, die in der Regel singuläre RehearsalProzesse zeigen, Sets mit 16 kategorisierbaren, hochtypischen Items entweder geblockt oder in einer Zufallsreihenfolge seriell dargeboten. Als weitere experimentelle Bedingung wurde das Interstimulusintervall variiert. In der einen Bedingung betrug die Präsentationsrate 4 Sekunden, in der anderen Bedingung belief sich der Interstimulusintervall nach jedem vierten Item auf $4+10$ Sekunden. Entgegen der Vorhersage konnte kein positiver Einfluss der ge- 
blockten Darbietung oder zusätzlicher Lernzeit auf die Anzahl der kumulativen Rehearser verzeichnet werden. Dagegen konnten frühere Befunde repliziert werden, die zeigten, dass die meisten Drittklässler unter Standardbedingungen singuläres Rehearsal nutzen und dass eine geblockte Darbietung zu höheren Reproduktionsleistungen führt, die jedoch nicht durch veränderte Rehearsal-Prozesse vermittelt werden. Um zu kontrollieren, ob die Kinder, die in diesem Experiment als transitional klassifiziert wurden, sich tatsächlich im Übergangsstadium zu der qualitativ höheren Stufe des kumulativen Rehearsals befanden, wurde knapp die Hälfte der Kinder ein Jahr später unter denselben Bedingungen nochmals getestet. Tatsächlich konnte Experiment 3 belegen, dass die Kinder, die ein Jahr früher als im Übergangsstadium befindlich eingestuft worden waren, mit größerer Wahrscheinlichkeit als die singulären Rehearser nun unabhängig von der Bedingung kumulatives Rehearsal zeigten. Somit stellte das frühe Verhalten unter unterstützender Bedingung einen guten Prädiktor für den weiteren Strategieerwerb und die Nutzung strategischer Prozesse unter weniger unterstützenden Kontexten dar.

Die Ergebnisse der Arbeitsgruppe um Ornstein und Guttentag liefern somit substantielle Belege für einen kausalen Zusammenhang zwischen der Qualität der Rehearsal-Aktivität, operationalisiert durch die Anzahl unterschiedlicher, während eines Interstimulusintervalls memorierter Items, und der Lernleistung. Der Rehearsal-Recall-Zusammenhang ist jedoch durch Randbedingungen eingeschränkt (vgl. Ornstein \& Naus, 1978, 1983), die die Durchmischung der Rehearsal-Sets (vgl. Cuvo, 1975) und die maximale Anzahl von Items in einem Rehearsal-Set betreffen. Bereits Naus et al. (1977) bemerkten in ihrer Untersuchung, dass die Lernleistung der Sechstklässler nicht durch eine Erhöhung des Rehearsal-Sets über etwa 3.5 Items gesteigert werden konnte (vgl. Ornstein \& Naus, 1978). In einer späteren Untersuchung fanden Ornstein und Naus (1983) ebenfalls Belege, dass die Lernleistung von Personen, die sechs oder sieben Items memorierten, nicht besser war als die von Strategen, die drei oder vier Items zu einem Rehearsal-Set zusammenfassten. Danach scheint die optimale Größe eines kumulativen Rehearsal-Sets bei drei bis vier (maximal fünf) Items zu liegen. Ornstein und Naus (1983, S. 421f) resümieren: „In addition, any statement that rehearsal activity facilitates recall needs to be qualified both by the specification of boundary conditions indicating limits on the number of items that can be effectively rehearsed together and by the possible importance of within-rehearsal-set variation in item position".

Die Lokation und Funktion von Rehearsal-Prozessen: Während kategoriales Organisieren und Elaboration sowohl zu den Enkodier- also auch zu den Abrufstrategien gezählt werden, treten Rehearsal-Prozesse lediglich während der Lernphase auf. Allerdings ist diese Zu- 
ordnung in der letzten Zeit kritisch diskutiert worden. Brown und Hulme (1995, S. 599) fordern beispielsweise: „If rehearsal does exert an important causal influence on memory span, it is clearly necessary to know exactly when such rehearsal is taking place". Theoretisch können Rehearsal-Prozesse in der Abrufphase nur während der aktuellen Outputreaktion oder während des Intervalls, das der Abrufphase vorausgeht, stattfinden. Ersteres ist sehr unwahrscheinlich, wenn man bedenkt, dass Rehearsal-Prozesse während der Abrufphase einen nachteiligen Effekt hätten, da sie die parallele Koordination zweier Sets, des Rehearsal-Sets und des Abruf-Sets, erfordern würden (Avons, Wright \& Pammer, 1994). Da das Intervall direkt vor der Abrufphase weder bedeutsame Korrelationen mit der Gedächtnisspanne noch mit der Artikulationsrate zeigt (z.B. Tehan \& Lalor, 2000), ist auch hier wenig Evidenz für die Annahme von output-basierten Rehearsal-Prozessen vorhanden (vgl. Nairne, 2002).

Neben der Lokation wird auch die Funktion von Rehearsal-Prozessen als Mechanismus zur re-presentation des Stimulusmaterials in neueren Arbeiten diskutiert. Nairne (2002) vermutet, dass durch Rehearsal-Prozesse Kopien der Lernitems im Gedächtnis produziert werden, die den späteren Abruf bestimmen. Multiple Repräsentationen, wie sie durch das kumulative Rehearsal erzeugt werden ,would be expected to increase the variability of contextual encoding and effectively shorten the functional retention interval" (Nairne, 2002, S. 63; s.a. Tan \& Ward, 2000). Nach dieser Sichtweise liegt der Nutzen von aktiven RehearsalProzessen weniger in der Auffrischung verblassender Gedächtnisspuren (s.u., Abschnitt 2.3.1), sondern eher in der Verbesserung des Erfolges von Cue-basierten Abrufprozessen also in Aktivationsprozessen im episodischen Gedächtnis. Ward (2001, 2002; Tan \& Ward, 2000) konnte zeigen, dass der Abruf in einer Free-Recall-Aufgabe bestimmt wird „by the number, distribution, and recency of items in rehearsal“" (Ward, 2002, S. 886) und dass Primacy- und Recency-Effekte beide durch einen recency-basierten Mechanismus erklärt werden können: Wenn die Reproduktionkurve nach der Position, an der ein Wort zuletzt memoriert wurde, und der Anzahl von Wörtern, die danach memoriert wurden, berechnet wird, zeigt sich ein großer Recency-Effekt und ein sehr geringer Primacy-Effekt. Die Präsentation eines Items und jeder Wiederholprozess (im Sinne eines Mini-Recalls) führen danach zu einer Erweiterung von assoziativen Hinweisreizen und erhöhen die Stärke des Items im episodischen Gedächtnis. Das Item mit der größten Gedächtnisstärke wird dann in der Lernphase zuerst abgerufen. Nach der Nennung eines Items irgendeiner Position werden Items genannt, die durch semantische Verbindungen oder durch die Konklusion in einem oder mehreren Rehearsal-Sets mit diesem Item assoziativ verknüpft sind - oft Items, die in der seriellen Präsentation unmit- 
telbare Nachbarn waren (Howard \& Kahana, 2001). Je mehr Zugangswege zu einem Item bestehen, umso wahrscheinlicher ist es, dass es genannt wird.

\subsection{Entwicklungsmodelle strategischer Gedächtnisprozesse}

Zahlreiche Studien haben Belege dafür erbracht, dass die bedeutsamsten und schnellsten Entwicklungsveränderungen bei den Gedächtnisstrategien im Verlauf der Grundschulzeit stattfinden. Allerdings lassen sich einzelne Strategien auch innerhalb eng definierter Entwicklungsstufen eher als situativ variable, kontextabhängige kognitive Prozesse mit großer interindividueller Streuung charakterisieren (vgl. Folds, Footo, Guttentag \& Ornstein, 1990). In den sechziger und siebziger Jahren wurden viele Untersuchungen durchgeführt, um die relevanten Entwicklungsschritte für einen effektiven Strategiegebrauch zu identifizieren. Dabei fokussierte man weniger auf strategische Kompetenzen von Kindern, sondern auf Defizite, also Phänomene, die erklären sollen, warum Kinder eine mnemotechnische Strategie (noch) nicht oder (noch) wenig effektiv anwenden. Heute werden drei Phasen als Vorläufer effizienten strategischen Verhaltens im Vor- und Grundschulalter diskutiert. In ihrer Gesamtheit legen die Untersuchungen zu den unterschiedlichen Strategien die Existenz einer generellen Entwicklungssequenz für mnemotechnische Strategien nahe. Diese Klassifikation suboptimaler strategischer Verhaltensformen weist allerdings eher einen deskriptiven als einen erklärenden Charakter auf.

\subsubsection{Mediationsdefizit}

Bereits in der zweiten Hälfte der sechziger Jahre beschäftigte sich die Arbeitsgruppe um Flavell (zusammengefasst bei Flavell, 1970) mit der Rolle innerer Mediatoren bei der Enkodierung und Reproduktion verbaler Informationen. Mediatoren sind kognitive Aktivitäten, die die Effizienz kognitiver Prozesse verbessern (vgl. Hasselhorn, 1996). Die erste Phase des Strategieerwerbs wird deshalb als Mediationsdefizit (mediational deficiency) bezeichnet, weil Vorschulkinder oft noch nicht über die notwendigen kognitiven Voraussetzungen verfügen, die einen Strategiegebrauch ermöglichen (Reese, 1962). Genau genommen sind die Kinder in diesem Stadium entweder nicht in der Lage, einen geeigneten inneren Mediator zu produzieren, oder aber der generierte Mediator erweist sich im Hinblick auf die Lern- und Gedächtnisleistung als ungeeignet. Auch ein Training oder die explizite Instruktion zur Anwendung einer Strategie können dieses Defizit nicht überwinden. 
Viele Studien haben die Beeinflussbarkeit der Rehearsal-Qualität durch Training thematisiert. Ein Anstieg der Leistung durch Training ist ein weiterer Beleg des Zusammenhangs zwischen Rehearsal-Prozessen und Leistung und zeigt weiterhin, dass die passiveren Rehearsal-Techniken jüngerer Kinder nicht auf ein Strukturdefizit zurückzuführen sind. Obwohl verschiedene Studien unterschiedliche Ergebnisse erbracht haben (z.B. Hagen, Hargrave \& Ross, 1973; Keeney, Cannizzo \& Flavell, 1967; Kingsley \& Hagen, 1969), kann doch unter ganz bestimmten Bedingungen eine Verbesserung der Leistung durch Training schon bei Vorschulkindern erzielt werden (z.B. Bernbach, 1967). Allerdings verweisen einige Autoren darauf, dass ein solches Training oft nicht vollends erfolgreich ist und ohne zeitlichen oder Situations-Transfer verläuft (z.B. Cowan, Saults, Winterowd \& Sherk, 1991). Insgesamt scheint es jedoch möglich, die Anwendung basaler Memorierprozesse bei Vorschulkindern durch prompting oder durch die Verwendung einfacher Aufgaben, mit geringen kognitiven Anforderungen, zu stimulieren. Somit kann in Bezug auf das Labeling und das singuläre Wiederholen bei Vorschulkindern schon nicht mehr von einem generellen Mediationsdefizit ausgegangen werden. Baker-Ward, Ornstein und Holden (1984) konnten in ihrer Studie bereits bei vier- und fünfjährigen Kindern memorization activities beobachten, die weit über das bislang mit dieser Altersgruppe verbundene Anschauen oder Anfassen der zu lernenden Objekte hinausgingen. In vielen Studien zeigen Kinder jedoch weniger ein Mediations- als vielmehr ein Produktionsdefizit (Miller \& Seier, 1994).

\subsubsection{Produktionsdefizit}

Die meiste Forschung wurde nicht zum Mediationsdefizit, sondern zum Produktionsdefizit (production deficiency; z.B. Flavell, 1970) durchgeführt. Ein Produktionsdefizit besteht, wenn Kinder eine Strategie zwar nicht spontan nutzen, wohl aber - und das mit einem gewissen Gewinn - wenn sie dazu angewiesen werden. Kinder in dieser zweiten Entwicklungsphase besitzen also durchaus die Fähigkeit, jedoch noch nicht die Bereitschaft zum Strategiegebrauch. Die Annahme eines Produktionsdefizits bedeutet dabei nicht, dass sich der Strategieerwerb nach einem Alles-oder-Nichts Prinzip gestaltet. Tatsächlich ist es wahrscheinlicher, dass die Akquisition und Anwendung von mnemotechnischen Strategien graduelle Prozesse sind und spontaner sowie effizienter Strategiegebrauch erst nach einiger Zeit und viel Übung erreicht wird (Ornstein et al., 1988; Ornstein, Medlin, Stone \& Naus, 1985).

Verschiedene Trainingsstudien konnten aufweisen, dass bereits Zweitklässler, die spontan nur wenige Worte wiederholen, mit Gewinn zum aktiveren Memorieren instruiert werden 
können (z.B. Kunzinger, 1985; Ornstein, Naus \& Stone, 1977), obwohl sie noch keinen zeitlichen Transfer der erlernten Strategie zeigen (Hagen et al., 1973). In der Studie von Kennedy und Miller (1976) führte bereits die bloße Rückmeldung „My goodness, you did so much better when you whispered those names over and over. I guess whispering helped you remembering the pictures better. Right?“،, im Sinne eines Feedbacks über Ursache-FolgeZusammenhänge, zur Anwendung einer aktiveren Rehearsal-Strategie mit einhergehendem Leistungsgewinn bei Sechsjährigen. Auch die Verringerung von mentaler Anstrengung bei einer Lernaufgabe durch die visuelle Verfügbarkeit aller Items bis zur Beendigung der Lernphase (z.B. Guttentag et al., 1987) und/oder die Gabe zusätzlicher Lernzeit (Naus, Ornstein \& Kreshtool, 1977; Ornstein et al., 1985) kann zur Nutzung vorhandener mnemotechnischer Kompetenzen führen. Dies zeigt, dass sich die Kinder in den frühen Grundschuljahren der Bedeutung aktiverer Memoriertechniken durchaus bewusst sein können. Sie sind jedoch weniger bewandert als ältere Kinder, aktivere Rehearsal-Prozesse spontan zu initiieren und dafür frühere Items aus dem Gedächtnis abzurufen (Baker-Ward et al., 1984; Ornstein et al., 1985).

\subsubsection{Nutzungsdefizit}

Aufbauend auf ihren Forschungsarbeiten kommen Miller und Bjorklund (z.B. Bjorklund, Miller, Coyle \& Slawinski, 1997) zu dem Schluss, dass die Entwicklung mnemotechnischer Strategien in vier qualitativ unterschiedlichen Phasen verläuft:

- In der ersten Phase scheitern die Kinder bei der Produktion einer Strategie (Mediationsdefizit).

- In der zweiten Phase gelingt es den Kindern nur partiell, die Strategie zu produzieren (Produktionsdefizit).

- Die dritte Phase ist durch ein Nutzungsdefizit (utilization deficiency) charakterisiert: Die Kinder nutzen die Strategie zwar spontan, jedoch ohne einen entsprechenden Leistungsgewinn.

- In der vierten Phase sind die Kinder in der Lage, die Strategie spontan und effektiv zu nutzen.

Das so genannte Nutzungsdefizit stellt einen relativen Newcomer unter den strategischen Defiziten dar. Dieser Begriff wurde von Miller $(1990,1994)$ in die Literatur eingeführt. Ein Nutzungsdefizit liegt vor, wenn ein Kind ,spontaneously produces an appropriate strategy, but accures no benefit from it for recall or less benefit than does an equally strategic older child“ (Miller \& Seier, 1994, S. 108). Nach Miller (1990) handelt es sich beim Nut- 
zungsdefizit um eine Übergangsphase zwischen keinem Strategiegebrauch und der effektiven und profitablen Anwendung einer mnemotechnischen Strategie.

Da Kinder zu unterschiedlichen Zeiten in unterschiedlichen Aufgaben zu effizienten Strategen werden, kann ein Nutzungsdefizit zeitlich variierend bei verschiedenen Aufgaben oder verschiedenen Versionen derselben Aufgabe auftreten. Besonders wenn Kinder erstmals spontan eine Strategie produzieren, kann dies zu a) einer Leistungsverminderung, b) keiner Leistungsveränderung oder c) einer vergleichsweise geringen Leistungssteigerung führen. Da also auch eine, relativ zu älteren Kindern, geringe Leistungsverbesserung als Nutzungsdefizit klassifiziert wird und es somit eher um einen Mangel an Effizienz des gezeigten strategischen Verhaltens als um ein strategisches Defizit im Sinne des Fehlens strategischer Prozesse geht, plädiert Hasselhorn (1996) für den Begriff der geringen Nutzungseffizienz.

In einer ausführlichen Literaturdurchsicht fanden Miller und Seier (1994) 41 Studien, die partielle oder deutliche Evidenz für ein Nutzungsdefizit über eine ganze Reihe von Strategien und Aufgaben hinweg liefern. Die Autoren nennen acht Studien, die die Annahme der Existenz eines Nutzungsdefizits für Rehearsal-Strategien stützen (Allik \& Siegel, 1976; Appel et al., 1972; Baker-Ward et al., 1984; Bray, Hersh \& Turner, 1985; McGilly \& Siegler, 1989; Neimark, 1976; Newman, 1990; Yussen, Gagne, Gargiulo \& Kunen, 1974). Drei Studien liefern uneinheitliche Belege (Beuhring \& Kee, 1987; Pressley \& Levin, 1977; McGilly \& Siegler, 1990), und nur die Studien von Weissberg und Paris (1986) sowie von Flavell et al. (1966) werden als Untersuchungen verbucht, die entgegengesetzte Evidenz bieten. Die letzten fünf Studien fanden entweder bessere Lernleistungen jüngerer Strategen gegenüber jüngeren Nichtstrategen oder keine signifikanten Altersunterschiede in der Lernleistung bei gleich strategischen Kindern (vgl. Miller \& Seier, 1994, S. 124).

Während Miller (1990, 1994) den Begriff Nutzungsdefizit ursprünglich explizit auf den untrainierten, spontanen Strategieeinsatz beschränkt hat, erweiterten Bjorklund und Coyle (1995) sowie Bjorklund et al. (1997) ihn auf vergleichbare Phänomene in Trainingsstudien. Obwohl das Nutzungsdefizit also scheinbar ein oft vorkommendes Phänomen darstellt, wird gerade diese Allgemeingültigkeit in neueren Studien in Frage gestellt (z.B. Hasselhorn, Richter \& Lingen, submitted; Schlagmüller \& Schneider, 2002; Sodian \& Schneider, 1999). Waters (2000) verweist weiterhin auf ein Definitionsproblem, das mit der bisherigen Konzeption verbunden ist. Ihrer Ansicht nach ist die bisherige Definition zu weit und zu unscharf, da in ihr mehrere Grade von strategischer Ineffizienz enthalten sind. Dadurch werde ein Problem bei der Differenzierung zwischen effizienter und ineffizienter Strategienutzung erzeugt und die Anzahl von Kindern, die als nutzungsdefizitär klassifiziert werden, überschätzt (vgl. auch 
Miller, 2000). Eine begrenztere Redefinition würde dagegen bedeuten, nur die Kinder als nutzungsdefizitär einzustufen, die in Relation zu gleichaltrigen Kindern keinen Effekt aus der Strategieanwendung erzielen (z.B. Schlagmüller \& Schneider, 2002; Waters, 2000), da die überlegenen Leistungen älterer Kinder auch durch nichtstrategische Prozesse wie die größere Informationsverarbeitungsgeschwindigkeit oder eine elaborierte Wissensbasis bedingt sein können.

\subsubsection{Multipler und flexibler Stategieeinsatz}

Die bisherigen Ausführungen können den Eindruck erwecken, dass Kinder bei einer Aufgabe immer nur eine Strategie anwenden und diese lediglich durch eine bessere bzw. effizientere Form ersetzen. Neuere Studien haben jedoch gezeigt, dass diese Sicht zu einfach ist. Kinder nutzen vielmehr eine Variation verschiedenster Strategien, um ein bestimmtes Problem zu lösen. Manchmal verwenden sie sogar mehrere Strategien zeitgleich. Auf der Grundlage mikrogenetischer Untersuchungen konnte die Arbeitsgruppe um Siegler (1996; Siegler \& Crowley, 1991; Siegler \& Jenkins, 1989; Siegler \& Stern, 1998) nachweisen, dass die traditionelle Sicht von Strategieentwicklung als linearem Verlauf zu größerer Effizienz nicht mit den Befunden einer großen Variabilität in der Strategienutzung übereinstimmt.

Siegler (1996) kritisiert das traditionelle Stufenmodell der kognitiven Entwicklung und weist darauf hin, dass dieses möglicherweise ein Artefakt darstellt. Seiner Ansicht nach resultieren Stufenmodelle aus der Art und Weise, wie Entwicklungspsychologen ihre Daten erheben - auf der Suche nach dem Weg der kognitiven Entwicklung (vgl. Taatgen, 1999). Nach Siegler (1996) generieren Kinder im Laufe ihrer kognitiven Entwicklung eine große Vielfalt von Strategien, um Probleme zu lösen. Je nach Art der aktuellen Aufgabe und den jeweiligen Zielen des Kindes werden dann bestimmte Strategien ausgewählt und häufiger genutzt. Andere, weniger effektive, werden dagegen seltener verwendet. Obwohl die Studien von Siegler (1996) nicht direkt auf die Gedächtnisentwicklung bezogen sind, scheinen seine Erkenntnisse doch auf diese übertragbar zu sein (z.B. Blöte, Resing, Mazer \& Noort, 1999). Die zentrale Botschaft ist dabei, dass Kinder zu jedem beliebigen Zeitpunkt und für jede Aufgabe eine Vielfalt von Strategien verfügbar haben (z.B. Coyle \& Bjorklund, 1997; Coyle, Read, Gaultney \& Bjorklund, 1999; Hock, Park \& Bjorklund, 1998).

Bereits 1977 betonten Ornstein, Naus und Miller die Notwendigkeit, die separaten und kombinierten Effekte von Rehearsal- und Organisationsstrategien zusammen zu erforschen. Ein Vergleich dieser beiden Gedächtnisstrategien bei Kindern der Klassenstufen zwei, drei 
vier und sechs wurde von Hasselhorn und Lindner-Müller (1995) durchgeführt. Bei der von den Autoren verwendeten Sort-Recall-Aufgabe zeigten erst Viertklässler (29\%) die Kombination beider Strategien. Die kombinierte Nutzung wies auf dieser Altersstufe jedoch gegenüber dem ausschließlichen Rückgriff auf das kategoriale Organisieren keinen Leistungsvorteil auf. Die Sechstklässler zeigten zu 50 Prozent beide Strategien während der Lernphase und hatten auch eine höhere Reproduktionsleistung als bei der Verwendung nur einer der beiden Strategien.

Cox, Ornstein, Naus, Maxfield und Zimler (1989) verglichen die kombinierte und spontane Anwendung von verbalem Rehearsal und kategorialem Organisieren. In einem ersten Experiment wurden Kinder der dritten und sechsten Klasse angewiesen, eine altersentsprechende und eine altersuntypische Rehearsal- und Sortierstrategie zu verwenden. Auf beiden Altersstufen führte die Verwendung fortgeschrittener, für Drittklässer untypischer Techniken zu besseren Leistungen. Im zweiten Experiment wurde Drittklässlern taxonomisch zusammengehöriges oder unverbundenes Lernmaterial unter vier experimentellen Bedingungen einer Free-Recall-Aufgabe dargeboten. Entweder wurde das jeweilige Item nur für fünf Sekunden oder während der ganzen Lernphase gezeigt. Weiterhin sollten die Kinder das Lernmaterial entweder nach seiner Bedeutung sortieren oder so, dass es ihnen beim Erinnern helfe. Die Kinder, die das Lernmaterial semantisch sortieren sollten, zeigten unabhängig von weiteren Bedingungen bessere Lernleistungen und aktivere Rehearsal-Prozesse. Cox et al. (1989, S. 626) bemerkten weiterhin: „In the conditions in which the children both sorted and rehearsed, the use of sorting appears to affect the efficency with which a subset of children will rehearse actively". Insgesamt scheint die Reflexion, die die Anwendung einer Strategie erfordert, den Gebrauch (aber nicht die Auswahl) einer anderen Strategie zu erleichtern. Kinder, die eine Gedächtnisstrategie für sich entdeckt haben und spontan einsetzen, können danach eine zweite Strategie effizienter nutzen.

Weitere Beispiele eines flexiblen Strategieeinsatzes enthält die Studie von McGilly und Siegler (1989). Kindergartenkinder, Zweit- und Drittklässler sollten dabei Reihen von seriell präsentierten Zahlenabfolgen in korrekter Reihenfolge wiedergeben. Die Kinder nutzten eine Vielfalt unterschiedlicher Strategien bei dieser Aufgabe, wobei jedes Kind eine Kombination verschiedener Strategien über mehrere Durchgänge zeigte. Selbst unter den Kindergartenkindern gebrauchte die Mehrheit bisweilen die effektivste Strategie, nämlich das kumulative Rehearsal. Veränderungen in der Wahl der Strategie richteten sich danach, ob ein Kind durch fehlendes Rehearsal im letzten Durchgang schlechtere Leistungen erbracht hatte. Entwicklungsveränderungen zeigten sich in der Rehearsal-Häufigkeit, in der Exaktheit der Strategien- 
anwendung und in der Wahl des am häufigsten genutzten Rehearsal-Typs (vgl. auch McGilly \& Siegler, 1990).

Diese Befunde decken sich mit denen aus Längsschnittstudien gewonnen Daten über relativ geringe Langzeit-Stabilitäten strategischen Verhaltens bei einer Sort-Recall-Aufgabe (Weinert \& Schneider, 1999). Die längsschnittliche Analyse der Entwicklung von Lernleistungen und Strategieverhalten auf der Ebene der Gruppenmittelwerte entsprach dabei dem Muster, das von Studien auf der Grundlage von Altersvergleichen bekannt war. Eine genauere Analyse ergab jedoch keine Evidenz für einen solchen graduellen und nahezu linearen Entwicklungsprozess. Stattdessen waren die individuellen Entwicklungsverläufe der Strategieentwicklung eher durch Sprünge und U-förmige Verläufe gekennzeichnet (Schneider \& Sodian, 1997). Insgesamt zeigt sich, dass die Daten über die Strategie-Akquisition aus Querschnittsuntersuchungen (die sich auch in längsschnittlichen Gruppenvergleichen widerspiegeln) individuelle Entwicklungspfade und -variabilitäten verschleiern. Die Resultate aus der Analyse dieser individuellen Entwicklungsmuster hinterfragen und verändern die traditionelle Sichtweise vom Erwerb mnemotechnischer Strategien.

\subsection{Determinanten der Gedächtnisentwicklung}

Die Entwicklung der Leistungsfähigkeit des Gedächtnisses lässt sich nicht alleine durch die Entwicklung der verbalen Gedächtnisstrategien erklären. Neben den mnemotechnischen Strategien gibt es noch eine Reihe weiterer Faktoren, die in ihrer Kombination bedeutsame Auswirkungen auf die Gedächtnisentwicklung und besonders die Entwicklung von Gedächtnisstrategien haben (Bjorklund, 2000). Genau genommen ergeben sich die Strategien erst aus dem Zusammenspiel weiterer Faktoren, wie der Gedächtniskapazität, dem bereichsspezifischen Vorwissen in der Wissensbasis und dem (allgemeinen und strategiespezifischen) Metagedächtnis (vgl. Hasselhorn, 1992). Diese verschiedenen Aspekte tragen in unterschiedlichem Ausmaß und zu unterschiedlichen Zeiten zur Gedächtnisentwicklung bei. Durch die frühe Zunahme der Verarbeitungsgeschwindigkeit und des Inhaltswissens steigt die funktionale Gedächtniskapazität. Etwas später als diese grundlegenden Prozesse haben die metakognitiven Fähigkeiten einen substantiellen Einfluss auf die Anwendung von mnemotechnischen Strategien und dadurch auf die Gedächtnisentwicklung (vgl. Siegler, 2001). Motivationalen Faktoren und dem Selbstkonzept eigener Fähigkeiten werden darüber hinaus mediierende Einflüsse auf die Entwicklung verschiedener Prozesse zugesprochen. 


\subsubsection{Das Kurzzeitgedächtnis und die Gedächtniskapazität}

Die flexible Fähigkeit, Informationen speichern und verarbeiten zu können, wird Kurzzeitgedächtnis oder Arbeitsgedächtnis (working memory) genannt. Sie ist von größter Bedeutung für unsere effektiven kognitiven Leistungen. Die limitierte Kapazität des Kurzzeitgedächtnisses bestimmt, wie viel Informationseinheiten zu einem bestimmten Zeitpunkt kurzfristig behalten werden können. Die durch die Gedächtniskapazität gesetzte Obergrenze kognitiver Leistungen wird durch qualitative Entwicklungsfortschritte verändert, die verfügbar werden, sobald die Verarbeitungskapazität groß genug ist (vgl. Goswami, 2001). Gedächtnisstrategien, metakognitives Wissen und Hintergrundwissen können also nur innerhalb der durch die limitierte Kapazität des Kurzzeitgedächtnisses gegebenen Beschränkungen wirken (vgl. Pressley \& Schneider, 1997; Schneider \& Pressley, 1997).

Das Kurzzeitgedächtnis ist seit der kognitiven Wende in den fünfziger Jahren ein wichtiger Gegenstand der Forschung. Seinerzeit glaubte man, es habe zwei Funktionen: Material bereitzuhalten, das wir innerhalb weniger Sekunden erinnern müssen, und als Zugang zum Langzeitgedächtnis zu fungieren (vgl. Smith, 1999). Die Theorie der Zugangsfunktion des Kurzzeitspeichers wurde in der Folgezeit jedoch teilweise durch die Existenz von Patienten unterhöhlt, deren Funktion des Kurzzeitgedächtnisses geschädigt war, deren Langzeitgedächtnis jedoch normal funktionierte (z.B. Shallice, 1988; Shallice \& Warrington, 1970). Kognitionswissenschaftler nehmen heute eher an, dass es die Hauptaufgabe des betreffenden Systems ist, bei Problemlösungen und Entscheidungen vorübergehend die Resultate von Zwischenberechnungen zu speichern (z.B. Baddeley, 1986). Angesichts dieser Aufgabe wurde dem betreffenden System der aus der Computerwissenschaft übernommene Name Arbeitsgedächtnis gegeben. Die Erforschung dieser Gedächtniskomponente wird als entscheidend nicht nur für die Analyse des Gedächtnisses angesehen, sondern für das Verstehen des Denkens selbst (Baddeley, 1999; Goswami, 2001).

In den frühen Gedächtnismodellen war das Kurzzeitgedächtnis als einheitlicher Speicher konzipiert (z.B. Atkinson \& Shiffrin, 1968). Es gibt jedoch aus Datenerhebungen bei Erwachsenen nur wenig überzeugende Belege dafür, dass ein einziges Gedächtnissystem alle Aspekte der Kurzzeitgedächtnisleistung stützt. Sowohl experimentelle Arbeiten (z.B. Baddeley \& Hitch, 1974) als auch neuere neuropsychologische Untersuchungen von Hirngeschädigten und Neuroimaging-Studien in Verbindung mit verschiedenen Aufgaben des Kurzzeitgedächtnisses weisen darauf hin, dass anatomisch und funktional unterschiedliche Systeme dem Aufbewahren und Einprägen von phonologisch-verbalem und visuell-räumlichem Material dienen (Andrade, 2001; Gathercole, 1999; Smith, 1999). Im Arbeitsgedächtnis-Modell 
von Baddeley und Hitch (1974; Baddeley, 1986) ist der Kurzzeitspeicher daher aus mehreren Komponenten zusammengesetzt. Dies Modell betont den funktionalen Charakter des Kurzzeitspeichers, indem es erlaubt, Informationen zeitgleich im Gedächtnis zu halten und miteinander in Beziehung zu setzen (vgl. Baddeley, 1999). In der Standardversion besteht das Modell aus drei Hauptkomponenten: Eine zentrale Steuereinheit, die Zentrale Exekutive, überwacht und koordiniert die zwei untergeordneten Hilfssysteme und ist für die modalitätsunabhängige Verteilung der limitierten kognitiven Ressourcen und die Enkodierung zuständig. Es kann Informationen in die beiden Hilfssysteme einspeisen oder aus ihnen abrufen. Für die vorübergehende Speicherung von visuell-räumlichen Informationen ist der visuell-räumliche Speicher zuständig (engl. visuospatial sketchpad). Das zweite Hilfssystem, die artikulatorische Schleife (ab 1986 phonologische Schleife genannt), dient der Verarbeitung und Aufrechterhaltung akustischer Informationen.

Es gibt Evidenz für eine weitere Unterteilung der beiden Hilfssysteme in jeweils einen passiven Speicher und einen aktiven Rehearsal-Prozess. Die Belege sind am stärksten für das phonologisch-verbale Hilfssystem. Danach kann der phonologische Speicher sprachbasierte Informationen für eine Dauer von 1.5 bis 2 Sekunden speichern. Anschließend verblassen die Informationen, wenn sie nicht durch den artikulatorischen Kontrollprozess - basierend auf einem subvokalen Rehearsal-Prozess - aufrechterhalten und wieder eingelesen werden (vgl. Baddeley, 1986, 1999). Die Voraussetzung hierfür ist, dass die Zeit, die für das (interne) Artikulieren benötigt wird, kleiner als die Spurenzerfallszeit ist. Das Arbeitsgedächtnis-Modell betont, dass die Informationsverarbeitung durch Kapazitätsgrenzen und Zeitrestriktionen eingeschränkt ist und dass dementsprechend die Verarbeitungsgeschwindigkeit einen bedeutenden Faktor bei der Behaltensleistung darstellt (vgl. Schneider, 2000). Entsprechend der Modellvorstellung ist die verbale Gedächtnisspanne hier als die Anzahl von Items definiert, die über eine kurze Zeit in der phonologischen Schleife verbleiben kann. Neuroimaging-Studien untermauern die Zwei-Komponenten-Sicht des verbalen Arbeitsgedächtnisses (Henson, Burgess \& Frith, 2000) und sprechen für eine vergleichbare Speicher-Rehearsal Unterscheidung im visuell-räumlichen Hilfssystem (z.B. Awh et al., 1999). Auch für die bislang kaum erforschte Zentrale Exekutive werden Belege angeführt, die vermuten lassen, dass die zentrale Steuereinheit kein einheitliches System ist (z.B. Miyake et al., 2000). [Zu weiteren Fallstudien, Variationen des Modells und seiner Diskussion siehe Andrade (2001), Baddeley (2000, 2002), Miyake und Shah (1999).]

Zwischen dem 4. und dem 14. Lebensjahr zeigt sich sowohl ein drastischer Anstieg der Gedächtnisspanne als auch der Verarbeitungsgeschwindigkeit. Hulme, Thomson, Muir und 
Lawrence (1984) konnten zeigen, dass ein linearer Zusammenhang zwischen der Gedächtnisspanne und der Artikulationsgeschwindigkeit besteht. Andere Autoren (z.B. Brown \& Hulme, 1995) gehen auf der Grundlage von Simulationsmodellen eher von einem quadratischen $\mathrm{Zu}$ sammenhang zwischen diesen beiden Faktoren aus. Da die entsprechenden Kurvenverläufe jedoch in dem experimentell erfassbaren Bereich einen annähernd linearen Verlauf aufweisen, gibt es hier kaum Unterschiede zwischen den beiden Positionen. Es kann somit von einem generellen, annähernd linearen Anstieg der Geschwindigkeit mentaler Prozesse ausgegangen werden. Dies ermöglicht es älteren Kindern und Erwachsenen im Gegensatz zu jüngeren Kindern, zeitgleich mehr mentale Operationen im Arbeitsgedächtnis durchzuführen (vgl. Hale, 1990; Kail \& Salthouse, 1994; LeBlanc, Muise \& Blanchard, 1992). Obwohl derartige Befunde zu den klarsten und zuverlässigsten bei entwicklungspsychologischen Studien zählen, sind die Implikationen nicht so offensichtlich. Es gibt erhebliche Kontroversen darüber, ob die Zunahme der Geschwindigkeit auf die verstärkte Anwendung von Strategien, die größere Vertrautheit mit dem Lernmaterial oder eine möglicherweise reifungsbedingte Zunahme des Tempos per se zurückzuführen ist (vgl. Schneider, 2000). Neuere Analysen und Berechnungen legen nahe, dass der letzte Aspekt zentral ist (Kail, 1991; Miller \& Vernon, 1997). Danach gibt es einen alterskorrelierten Anstieg der Verarbeitungsgeschwindigkeit neben und über Zuwächse hinaus, die auf andere Faktoren zurückzuführen sind. Dies deckt sich auch mit Befunden von Case, Kurland und Goldberg (1982). Sie untersuchten, ob ein kausaler Zusammenhang zwischen der Verarbeitungsgeschwindigkeit und der Lernleistung von verbalem Lernmaterial besteht. Sie fanden bei der Verwendung von Material mit altersunabhängiger Artikulationsrate für Kinder und Erwachsene keine Unterschiede in der Lernleistung zwischen diesen Gruppen.

Allgemein lässt sich sagen, dass Entwicklungsverbesserungen in der Gedächtnisspanne sowohl durch einen Zuwachs an verfügbaren Ressourcen als auch durch eine Verringerung der zur Bearbeitung einer Aufgabe benötigten Ressourcen zustande kommen können (Siegler, 2001).

\subsubsection{Der Einfluss von Vorwissen}

Die Effizienz und Geschwindigkeit kognitiver Prozesse wird ebenfalls maßgeblich von dem Wissen beeinflusst, das eine Person über einen bestimmten Bereich hat (vgl. Cowan, 1997). Dieses Inhalts- bzw. Vorwissen im Langzeitgedächtnis spielt eine substantielle Rolle bei der Speicherung, dem Abruf und der Verarbeitung von Informationen. Nach der Netz- 
werktheorie der Wissensrepräsentation liegt es in Form eines mehrdimensionalen Netzwerkes aus Knoten und unterschiedlich starken Verbindungen zwischen diesen Knoten als proprotionales Repräsentationssystem im semantischen Gedächtnis vor. Umfangreiches Wissen in einem bestimmten Bereich ermöglicht es, Informationen aus diesem Bereich schneller zu verarbeiten, und wirkt sich entscheidend auf die Höhe der Behaltensleistung aus (Bjorklund, 2000). Die sich entwickelnde Wissensbasis ist damit ein zentraler Motor der Gedächtnisentwicklung. Dabei beeinflussen Unterschiede im bereichspezifischen Wissen das Gedächtnis im Wesentlichen auf drei Arten (Bjorklund, 1987; Pressley \& Schneider, 1997):

- Erstens sind bestimmte Informationen leichter zugänglich, da sie reichhaltiger und somit anschaulicher repräsentiert sind (z.B. Lindberg, 1980).

- Zweitens kann der Abruf von Lerninhalten durch die Aktivierung von Verknüpfungen zwischen Items im Langzeitgedächtnis nahezu automatisch erfolgen (Bjorklund \& Bjorklund, 1985).

- Drittens kann gut entwickeltes content knowledge den Gebrauch von Strategien und metakognitiven Prozessen unterstützen, die wiederum die Lernleistung verbessern (z.B. Bjorklund, 1987; Hasselhorn, 1995).

Evidenz für den starken Einfluss der Wissensbasis auf die Gedächtnisleistung erbringen Studien, die die Leistung von Personen mit wenig Vorwissen auf einem Gebiet (Novizen) mit der von Personen vergleichen, die sich in dem betreffenden Gebiet sehr gut auskennen (Experten). Chi (1978) verglich das Gedächtnis von zehnjährigen Schachexperten mit dem von erwachsenen Schachneulingen. Die Aufgabe bestand darin, die durchschnittlich 22 Figuren einer Mittelspielstellung möglichst genau nachzustellen, nachdem man sich diese zuvor 10 Sekunden anschauen durfte. Die Kinder konnten im Durchschnitt 9.3 Figuren korrekt positionieren, die Erwachsenen 5.9. Darüber hinaus brauchten die Neulinge bedeutend mehr Durchgänge, um sich die vollständige Konfiguration der Schachfiguren einzuprägen. Laut Chi (1978) erkannten die Experten sinnvolle Muster in der Aufstellung, was ihre Erinnerungsleistung verbesserte. Dementsprechend scheint der Vorteil der Experten sowohl einen qualitativen bzw. strukturellen (Art und Weise der Repräsentation) als auch einen quantitativen Aspekt zu besitzen (vgl. auch Chi, Hutchinson \& Robin, 1989; Lindberg, 1991; Schneider, Gruber, Gold \& Opwis, 1993). Untersuchungen von Ceci und Liker (1986) sowie Schneider, Körkel und Weinert (1989) zeigten weiterhin, dass bereichsspezifische Sachkenntnisse die Erinnerungsleistung sogar stärker beeinflussen können als die Intelligenz und dass bei der Bearbeitung bestimmter Anforderungen ein geringer Gesamt-IQ durch Expertise kompensiert werden kann. Nachfolgestudien verdeutlichten jedoch, dass bereichsspezifische Expertise 
vorhandene Intelligenzunterschiede nicht generell und vollständig eliminiert. Eine nicht vorhandene Begabung in einem Bereich kann aber durch Hintergrundwissen ausgeglichen werden (Schneider, Bjorklund, \& Maier-Brückner, 1996).

Eine elaborierte Wissensbasis verbessert nicht nur die Informationsverarbeitung und -speicherung, sondern optimiert auch die Aneignung und Ausführung von Strategien. Naus und Ornstein (1985) konnten z.B. nachweisen, dass studentische Fußballexperten bei auf Fußball bezogenem Lernmaterial nicht nur bessere Lernleistung erbrachten als Nichtexperten, sondern die Items auch eher kumulativ memorierten als die Novizen. Die Ergebnisse verschiedener Studien erbringen weiterhin Belege dafür, dass Kinder neue Strategien ebenfalls erstmals bei vertrauten Inhalten zeigen (z.B. Chi, 1981). Eine mögliche Erklärung dafür ist, dass die assoziativen Zusammenhänge bei bekanntem Material oder Inhalt größer sind und Kindern erlauben, bei Lernvorgängen auf reichhaltigere Verknüpfungen und Abrufpfade zurückzugreifen (vgl. Ornstein \& Naus, 1985). Dies wiederum ermöglicht eine tiefere und flexiblere Verarbeitung und wirkt sich positiv auf die Abrufleistung aus (z.B. Kee \& Davis, 1988, 1990). Tarkin, Myers und Ornstein (1985) untersuchten diese Hypothese, indem sie Achtjährigen vertrautes Lernmaterial gaben, das sich nur in seinem Bedeutungsgehalt unterschied. Es zeigte sich, dass eine größere Anzahl möglicher assoziativer Verknüpfungen innerhalb des Lernmaterials zur Bildung größerer Rehearsal-Sets führte (siehe hierzu auch Tarkin, 1981).

In einer weiteren Studie fanden Ornstein, Naus und Liberty (1975, Experiment 2), dass die geordnete Darbietung von taxonomisch zusammengehörigen Items (blocked pattern), verglichen mit einer Zufallsreihenfolge, zu einem Anstieg an kumulativem Rehearsal in der Lernphase führte. Unter der geordneten Präsentation nutzten sowohl die Sechst- als auch die Neuntklässler die kategoriale Struktur zur Bildung der Rehearsal-Sets. Bei der Zufallsreihenfolge konnten nur die Neuntklässler die implizite taxonomische Struktur in die Rehearsal-Sets aufnehmen. Für die beteiligten Drittklässler zeigte sich kein Einfluss der Darbietungsart auf die Wiederholungstechnik. Nach Ansicht der Autoren trat die Organisationsstruktur der Items durch die geblockte Präsentation deutlicher hervor, was das aktivere Rehearsal erleichterte und sich positiv auf die Lernleistung auswirkte. Ornstein, Naus und Miller (1977) replizierten und erweiterten das berichtete Befundmuster. Danach scheint ein aktiver Rehearsal-Prozess die Lernleistung nur dann zu beeinflussen, wenn die Strukturierung des Lernmaterials minimal ist (wie bei unverbundenen Items) oder nur schwer nutzbar zu machen ist (wie bei der randomisierten Darbietung taxonomisch verbundener Items). Dagegen rücken bei einer offensichtlichen Listenstruktur, wie bei der geblockten Darbietung semantisch zusammengehöriger Items, Rehearsal-Faktoren in den Hintergrund und werden somit relativ unwichtig. 
Verschiedene Studien konnten diesbezüglich zeigen, dass die Gedächtnisleistung bei gruppierter Präsentation derjenigen bei ungruppierter Präsentation sogar dann überlegen ist, wenn Memorierprozesse mittels artikulatorischer Unterdrückung verhindert werden (Frick, 1989; Hitch, Burgess, Towse \& Culpin, 1996). Gaultney, Bjorklund und Schneider (1992) resümieren, „that expert children's advantage on a strategic memory task is mediated primarily by nonstrategic factors“ (s.a. Schneider \& Bjorklund, 1992). Die Autoren bezweifelten dabei nicht, dass Experten sich unter Umständen strategischer bei einer bestimmten Aufgabe verhielten. Der Vorteil der Experten gegenüber den Novizen sei jedoch allgemein auf das Gebiet der Expertise beschränkt und basiere somit wesentlich auf nicht-strategischen Komponenten.

Die Ergebnisse verschiedener Studien legen nahe, dass Langzeitwissen in Form von lexikalischen Informationen auch genutzt wird, um während der Abrufphase die Repräsentation von Stimuli im phonologischen Kurzzeitspeicher aufzufrischen bzw. zu rekonstruieren (z.B. Brown \& Hulme, 1995; Hulme, Newton, Cowan, Stuart \& Brown, 1999; Hulme et al., 1997). Danach findet während der Abrufphase eine Art verdeckter Wiedergewinnungs-Prozess in den Sprechpausen statt, um das nächste Item abzurufen und die Gedächtnisspuren der übrigen Items zu reaktivieren. Beim Prozess der Mustervervollständigung verblassender Items (redintegration) wird durch einen Hinweisreiz eine Durchsuchung des Langzeitspeichers nach weiteren assoziativen Erinnerungen in Gang gesetzt (vgl. Gathercole \& Martin, 1996). Dieser strategische Prozess entwickelt sich mit zunehmendem Alter. Er wird nicht vor dem siebten Lebensjahr eingesetzt und ist vor allem bei längeren und vertrauten Items hilfreich (Turner, Henry \& Smith, 2000). Cowan $(1994,1997)$ geht davon aus, dass der Abgleichvorgang, den er als Suchprozess im Gedächtnis bezeichnet, bei älteren Kindern bzw. Kindern mit höherer Gedächtnisspanne effizienter verläuft als bei jüngeren Kindern oder solchen mit niedriger Gedächtnisspanne (vgl. Tehan \& Lalor, 2000) und dass es sich bei diesen scanning-basierten Suchprozessen um eine Art Reheasal handelt (Cowan, 1992).

Die höhere Assoziativität zwischen Items führt neben einer verbesserten Reproduktionsleistung auch zu kürzeren Interitemlatenzen in der Abrufphase. Bei kategorisierbaren Wortlisten sind die Interitemlatenzen (Inter-Response Times) zwischen Wörtern aus einer Kategorie daher kürzer als die zwischen Wörtern aus unterschiedlichen Kategorien (vgl. Howard \& Kahana, 2002). Dieses Phänomen wird als response bursting bezeichnet (Pollio, Richards \& Lucas, 1969). Die between-catogory Latenz ist dabei nach Patterson, Meltzer und Mandler (1971) durch drei Prozesse gekennzeichnet: die Beendigung des Abrufs von Items aus Kategorie A (category exit), das Aufrufen von Kategorie B (category access) und der Abruf von 
Items aus Kategorie B (word access). Die Wiedergewinnung von Items einer Kategorie findet vermutlich ebenfalls in Form eines re-access checks statt, bei dem jedes Element einer Kategorie darauf überprüft wird, ob es schon genannt wurde (vgl. Patterson et al., 1971). Die Geschwindigkeit der geschilderten Such- und Rekonstruktionsprozesse (in Items pro Sekunde) kann laut Cowan, Nugent und Elliott (2000) abgeschätzt werden „by dividing the number correct by the mean silent period“ (S. 667). Die berichteten Befunde zum Einfluss von Langzeitwissen auf die Gedächtnisleistung veranschaulichen, dass Vorwissen der Motor der Gedächtnisentwicklung ist. Die Reichhaltigkeit und Verknüpfung deklarativen und konzeptuellen Langzeitwissens in der Wissensbasis sowie die Leichtigkeit und Geschwindigkeit des Zugriffs auf diese Informationen bilden somit eine weitere wichtige Determinante der kognitiven Entwicklung.

\subsubsection{Die Bedeutung metakognitiver Prozesse}

Breit gefasst ist Metakognition (metacognition) ein Sammelbegriff für jegliches Wissen über Kognitionen und die Steuerung und Überwachung kognitiver Prozesse. Obwohl die historischen Wurzeln tiefer liegen (z.B. James, 1890), erhielt die Erforschung metakognitiver Prozesse erst durch die Arbeiten von Flavell in den siebziger Jahren weitgehende Beachtung. Diese Arbeiten befassten sich mit Entwicklungsveränderungen in den Erkenntnissen von Kindern über das Gedächtnis (metamemory), das Verständnis (metacomprehension) und die Kommunikation (metacommunication; z.B. Flavell, 1979). Auf der Grundlage zahlreicher Forschungsarbeiten werden metakognitive Prozesse inzwischen als wichtiger Faktor vieler kognitiver Aspekten angesehen (z.B. Gedächtnis, Aufmerksamkeit, Kommunikation, Problemlösen und Intelligenz; vgl. Moses \& Baird, 1999). In diesem Sinne stellen Metakognitionen als Kognitionen zweiter Ordnung oder „Wissen über Wissen“ eine bereichsübergreifende Facette dar (Kuhn, 2000).

Obwohl das Konzept Metakognition sehr heterogen und ,fuzzy" (Flavell, 1981, S. 37) ist und es Differenzen über die Charakterisierung der unterschiedlichen Teilaspekte von Metakognition gibt (vgl. Schneider \& Pressley, 1997), unterscheiden die meisten Autoren zwischen einer deklarativen Komponente, dem metakognitiven Wissen, und einer prozeduralen Komponente, der metakognitiven Regulation. Ersteres bezieht sich auf Informationen, die jemand über seine Kognitionen und Kognitionen im Allgemeinen besitzt. Flavell (1979; Flavell \& Wellman, 1977) differenziert das metakognitive Wissen weiter in

- Wissen über Personenmerkmale (z.B. zu wissen, dass man ein gutes Gedächtnis hat), 
- Wissen über Aufgabenmerkmale (z.B. zu wissen, dass kategorisierbare Items leichter zu behalten sind als unverbundene Items),

- Wissen über Gedächtnisstrategien (z.B. das Wissen über Organisations- oder Rehearsal-Strategien) und

- Wissen über die Interaktion dieser Faktoren (z.B. zu wissen, dass die Organisationsstrategie den Rehearsal-Strategien bei kategorisierbarem Material überlegen ist).

Die metakognitive Regulation umfasst dagegen eine Vielfalt von Exekutivfunktionen wie die Planung, Ressourcenallokation, Steuerung und Korrektur kognitiver Prozesse (Brown, Bransford, Ferrara \& Campione, 1983). Auch hier kann eine weitere Differenzierung in Überwachungs- und Steuerungsprozesse vorgenommen werden, je nachdem ob Informationen zum „Metalevel“ hin oder von ihm weg fließen (Nelson \& Narrens, 1990). Neuere NeuroimagingStudien betonen einen starken Zusammenhang zwischen der metakognitiven Kontrolle und exekutiven Kontrollfunktionen im Arbeitsgedächtnis (vgl. Shimamura, 2000) und benennen Areale im mittleren Frontalen Cortex als biologische Basis für metakognitive Regulationsprozesse (Fernandez-Duque, Baird \& Posner, 2000). Flavell berücksichtigt in seinen Arbeiten (z.B. Flavell \& Wellman, 1977) noch eine weitere Kategorie, die Sensitivitätskategorie, die ein „Gefühl zu Wissen“ (feeling of knowing) beinhaltet. Hierunter wird eine Art implizites, vorbewusstes Gefühl für die Möglichkeiten eigener kognitiver Aktivitäten verstanden. Der Sensitivitätskategorie wird von verschiedenen Autoren eine zentrale Bedeutung bei der Entwicklung und Anwendung neuer Gedächtnisstrategien zugesprochen (z.B. Hasselhorn, 1992; Kuhn, 2000). Nach Koriat (2000) fungieren die metakognitiven Gefühle als Verbindung zwischen den implizit-unbewusst-automatischen Prozessen und den explizit-bewusst-gesteuerten und überwachten Prozessen. Aufgrund der Unschärfe und Heterogenität des Metakognitionskonzeptes wurden im Laufe der Zeit eine große Anzahl von Klassifikationsversuchen unternommen (z.B. Cavanaugh, 1989; Flavell \& Wellman, 1977; Hasselhorn, 1992; Kuhn, 2000; O’Sullivan \& Howe, 1998)

Metakognitive Kenntnisse und Regulationsprozesse sind oftmals eng und bidirektional miteinander verflochten: Das Wissen, dass eine Aufgabe schwierig ist, kann eine Person z.B. zur sorgfältigen Überwachung ihrer kognitiven Prozesse verleiten. Umgekehrt können aus Überwachungsprozessen Erkenntnisse über die Aufgaben gewonnen werden. Eine zentrale Annahme der Metagedächtnisforschung ist, dass Kinder mit zunehmendem Metagedächtniswissen in verstärktem Maße aktiv mnemotechnische Strategien einsetzen und bewusst zwischen verschiedenen Gedächtnisstrategien wählen, was sich wiederum positiv auf die Lernleistung auswirken kann. Daher wird in einigen Metagedächtnismodellen besonders der $\mathrm{Zu}$ - 
sammenhang zwischen Metakognition und Strategien betont (z.B. Borkowski, Milstead \& Hale, 1988; Borkowski \& Turner, 1990). Das bewusste Wissen über spezifische, relationale und generelle Gedächtnisstrategien wird in diesen Modellen als wesentliche Komponente des Strategienerwerbs und ihrer Anwendung betrachtet (vgl. Pressley, Borkowski \& O’Sullivan, 1985; Schneider, 1999). Metaanalysen ermitteln jedoch allgemein einen mäßig hohen Zusammenhang zwischen Metagedächtnis und kognitiver Leistung (z.B. Schneider, 1985), der bei älteren Kindern und schwierigeren Aufgaben etwas stärker ausfällt. Die Korrelation zwischen Metakognition und Strategiegebrauch ist dabei höher als die zwischen Metakognition und Leistung. Obwohl es inzwischen eine Reihe von Modellen gibt (z.B. Kurtz \& Weinert, 1989; Schneider, Körkel \& Weinert, 1987), ist die exakte Form und die Stärke des Einflusses von Metakognitionen auf die Gedächtnisentwicklung und -leistung noch nicht abschließend beantwortet, und es ist zu bezweifeln, dass es den einzig richtigen Einflussweg gibt (vgl. Hasselhorn, 2001).

Zur Erfassung deklarativer metakognitiver Bewusstheit werden neben einigen anderen Methoden hauptsächlich Fragebögen oder Interviews eingesetzt. Diese enthalten Fragen zu verschiedenen Gedächtnisprozessen und Lernaufgaben. Viele Instrumente und Skalen weisen jedoch nur schlechte bis mittlere Gütekriterien auf (vgl. Schlagmüller, Visé \& Schneider, 2001). Darüber hinaus findet sich oftmals eine Konfundierung mit anderen Konstrukten wie dem Selbstkonzept und der Motivation (vgl. Hasselhorn, Hager \& Baving, 1989). Trotz einschlägiger Bemühungen zur Entwicklung ökonomischer Instrumente mit guten Gütekriterien wurden erst in neuester Zeit reliable und valide Fragebögen zur Erfassung des deklarativen Metagedächtnisses vorgestellt (z.B. Hasselhorn, 1994; Schlagmüller, Visé \& Schneider, 2001). Neben Items zum aufgabenbezogenen Wissen und allgemein-deklarativen Metagedächtnis enthalten diese Erhebungsinstrumente Fragen zum strategiespezifischen Metagedächtnis, die jedoch durchgängig auf die Organsiationsstrategie bezogen sind. Leider sind auch nahezu alle Studien zum Zusammenhang von metakognitiven Prozessen und Gedächtnisstrategien mit dem Fokus auf die Organisationsstrategie durchgeführt worden, so dass es fast keine Erkenntnisse zum Zusammenhang von Metakognitionen und Rehearsal-Prozessen gibt. Allerdings geht Kuhn (2000) davon aus, dass die Strategie-Metastrategie-Relation eine gewisse Allgemeingültigkeit über verschiedene kognitive Prozesse hinweg aufweist, speziell in der Form, in der Prozesse auf dem Metalevel die Auswahl und Regulation von Gedächtnisstrategien steuern.

Eine der wenigen Studien, die die Bewusstheit für Rehearsal thematisiert, stammt von Flavell, Green, Flavell und Grossman (1997). In zwei Untersuchungen belegen die Autoren, 
dass Vorschulkinder kaum Wissen und Bewusstheit über interne Sprachprozesse (inner speech) bei sich selbst oder anderen haben. Die Entdeckung dieser verdeckten mentalen Aktivitäten während des Lernens geschieht erst im Verlauf der ersten Grundschuljahre, obwohl bereits Fünfjährigen der Einsatz offener und verdeckter Rehearsal-Prozesse durch ein Training vermittelt werden kann (z.B. Johnston \& Conning, 1990). Das zunehmende Gewahrwerden interner Sprachprozesse und der Häufigkeit ihres Auftretens während kognitiver Aktivitäten geht mit ihrem bewussten Einsatz und der Entwicklung von Rehearsal-Strategien während der Grundschule einher (vgl. Flavell, Green \& Flavell, 2000). In einer anderen Untersuchung ließ Justice (1985) sieben-, neun- und elfjährige Kinder den Nutzen von Betrachten, Benennen, Rehearsal und semantischem Kategorisieren einschätzen. Kinder aller Altersstufen erkannten die Überlegenheit von Rehearsal und Organisation gegenüber Betrachten und Benennen. Weiterhin schätzten die Siebenjährigen Rehearsal und Kategorisieren als gleich wirksam ein. Erst die 9 und 11 Jahre alten Kinder betrachteten Kategorisieren als effektiver und zeigten damit, dass sie metakognitives Wissen über den Nutzen von semantischer Kategorisierung besitzen (vgl. auch Justice, 1986).

Diese Befunde decken sich mit den generellen Erkenntnissen über die Entwicklung metakognitiver Bewusstheit: Nach Flavell (1999) wissen bereits Zweijährige, dass man sich anstrengen muss, um sich etwas zu merken, und Dreijährige kennen den Unterschied zwischen ,an etwas denken“ und „sich etwas merken“. Bei Vorschulkindern findet man jedoch nur ansatzweise und bei vertrauten Lernanforderungen aufgaben- oder strategiebezogenes metakognitives Wissen. Dieses entwickelt sich erst im Laufe der Grundschulzeit in verschiedenen Bereichen, wie z.B. bei der Prognosegenauigkeit eigener Merkleistung, der Zeitallokation oder dem Wissen über die Nützlichkeit von Rehearsal- und Organisationsstrategien (z.B. Hasselhorn, Mähler \& Grube, 1995; Schneider, 1999; Schneider \& Sodian, 1988). Der Entwicklungsprozess kann sich allerdings noch weit bis ins Jugend- und frühe Erwachsenenalter hinein erstrecken.

\subsubsection{Motivationale Aspekte}

Das Wissen, dass eine Gedächtnisstrategie hilfreich ist, reicht jedoch alleine noch nicht aus. Kinder müssen auch motiviert sein, diese Strategie bei Lernanforderungen anzuwenden. Erst wenn ein Kind weiß, warum eine Gedächtnisstrategie hilfreich ist, und es den Nutzen verschiedener Strategien differenziert einschätzen kann, steigt die Motivation, die für eine Lernanforderung optimale Strategie anzuwenden (vgl. Sigelman, 1999, Kapitel 8). Leider sind 
jedoch in der Forschung Fragen der Motivation und Kognition oft separat behandelt worden (Pintrich \& Garcia, 1993). Weitgehend unabhängig von der theoretischen Konzeption formulieren verschiedene Autoren einen mediierenden Einfluss von Motivation auf Informationsverarbeitungsprozesse, speziell auf den Einsatz von Gedächtnisstrategien und auf Lernerfolg insgesamt (z.B. Baumert, 1997; Vollmeyer \& Rheinberg, 1998). Einige Forscher betonen die Notwendigkeit, motivationale Faktoren generell stärker zur berücksichtigen, da Gedächtnisstrategien als selbstinitiierte Aktivitäten Initiative und Auswahl beinhalten und es daher erzwungenermaßen einen engen Zusammenhang zwischen Gedächtnisstrategien und motivationalen Faktoren gibt (z.B. Pierce \& Lange, 2000). Bei diesen kann man zwischen intrinsischen und extrinsischen Faktoren unterscheiden.

Die zentrale Determinante der intrinsischen Motivation ist das Interesse (Schiefele, 1996). Interesse wird als spezifische Person-Gegenstands-Relation definiert und weist einen hohen Zusammenhang zur Anwendung von Gedächtnisstrategien auf (z.B. Baumert, 1997). Verschiedene Befunde weisen darauf hin, dass interessenorientiertes Lernen speziell mit einer tiefen Verarbeitung des Lernmaterials und der Nutzung entsprechender Gedächtnisstrategien einhergeht (z.B. Entwistle \& Ramsden, 1983; Nolan, 1988; Schiefele, 1989). Pierce und Lange (2000) fanden in ihrer Studie einen direkten Einfluss von motivationalen und metakognitiven Faktoren auf die Strategieanwendung und die Lernleistung bei Erwachsenen. Neuere Studien ergeben Evidenz dafür, dass auch Erwerb und früher Einsatz von Gedächtnisstrategien durch Interesse gefördert werden. Wenn dieses Interesse durch interessantes Spielzeug oder Lernmaterial hervorgerufen wird, kann das die Art von unterstützendem Kontext herstellen, in dem frühe Strategien erstmals auftreten (Bjorklund, 1987; Miller \& Seier 1994). Oyen und Bebko (1996) fanden wesentliche Hinweise, dass Interesse die Strategienutzung fördert. Die vier- bis siebenjährigen Kinder in ihrer Studie zeigten deutlich mehr kumulatives Rehearsal, wenn eine serielle Lernaufgabe in einem interessanteren Kontext angeboten wurde (Computerspiel vs. traditionellem Lernkontext). Dieses Ergebnis war altersunabhängig. Wie erwartet zeigte sich eine Zunahme an Rehearsal mit steigendem Alter. Weiterhin profitierten die Kinder aller Altersstufen (junior kindergarten, senior kindergarten, grade 1) vom Einsatz der Rehearsal-Strategien. Allerdings war hier die Lernleistung im traditionellen Lernkontext höher. Das Computerspiel war zwar interessanter, die Vielzahl von Distraktoren und Zielen innerhalb des Spiels machten es jedoch deutlich schwieriger als die traditionelle Lernaufgabe.

Eine extrinsische Motivation besteht darin, dass ein äußerer Anreiz als Belohnung für ein bestimmtes Verhalten gegeben wird. Dies taten Kunzinger und Witryol (1984) in ihrer Studie über den Einfluss unterschiedlich motivierender Anreize auf die Anwendung von Re- 
hearsal. Sie versprachen siebenjährigen Kindern für das Behalten bestimmter Wörter einer Lernliste 10 Cent und für das Behalten anderer Wörter 1 Cent. Es zeigte sich, dass die Kinder deutlich mehr Rehearsal-Aktivität auf die 10-Cent-Wörter verwendeten. In einer Kontrollbedingung, in der für jedes Wort fünf Cent gegeben wurde, wurde insgesamt weniger Rehearsal registriert. Weiterhin zeigte sich unter der ersten Bedingung ein Generalisierungseffekt: Die Kinder dehnten den Einsatz von Rehearsal-Strategien auf die 1-Cent-Wörter aus. Der verstärkte Einsatz von Rehearsal verbesserte darüber hinaus die Erinnerungsleistung der 10Cent-Wörter. Diese wurden signifikant öfter behalten als 1-Cent- oder 5-Cent-Wörter. Die gleichen Ergebnisse erbrachte eine Untersuchung mit Fünft- und Achtklässlern von Cuvo (1974). In einer Studie von O’Sullivan (1993) konnten bereits Vierjährige mit Hilfe attraktiver Anreize zum Einsatz von Gedächtnisstrategien angeregt werden. Die genannten Befunde und die Ergebnisse ähnlicher Studien zeigen, dass es Kindern oft eher an der Motivation als an der Fähigkeit zur Nutzung von Rehearsal mangelt. Insgesamt untermauert dies die Annahme, dass motivationale Faktoren, speziell Interesse, eine ähnlich große Rolle bei der Strategieentwicklung spielen wie das Hintergrundwissen (vgl. Bjorklund, 1987).

\subsubsection{Das Selbstkonzept}

Das Selbst-System einer Person ist ein komplexes, interdependentes System. Es beinhaltet so unterschiedliche Konstrukte wie die Selbstwirksamkeitsüberzeugung (self-efficacy), das Selbstwertgefühl (self-esteem), die Kontrollüberzeugung (locus of control), das Leistungsmotiv (achievment motivation) und die Attributionsüberzeugungen (attributional beliefs) einer Person (Borkowski, Carr, Rellinger \& Pressley, 1990). In der Gesamtheit stellt dieses „Selbst-Bild“ eine Art interne kognitive Repräsentation des Wissens und der Überzeugungen über sich selbst dar. Viele Autoren unterscheiden zwischen dem globalen Selbstkonzept und spezifischen Selbstkonzepten. Letztere beziehen sich dann auf die Beurteilung einer Person über ihre Leistung in einem bestimmten Bereich (vgl. Zanobini \& Usai, 2002). Dem SelbstSystem, speziell dem Selbstwertgefühl, wird ein bedeutender Einfluss auf metakognitive Funktionen und schulische Leistungen attestiert (z.B. Marsh, 1986; McCombs, 1986; Oka \& Paris, 1987). Das Fähigkeitsselbstkonzept umfasst die Gesamtheit der Gedanken bezüglich eigener Fähigkeiten (Meyer, 1984; Pekrun, 1983). Nach Helmke und Rheinberg (1996) ist das Selbstkonzept eigener Fähigkeiten ein motivationsrelevantes Persönlichkeitsmerkmal, das einen zentralen Faktor des Leistungsmotivs darstellt. Andere Autoren verweisen darauf, dass das Selbst-System der Entwicklung des metakognitiven Systems zugrunde liegt und in der 
Form eines Mediators über dieses die Qualität kognitiver (speziell schulischer) Leistungen beeinflusst (Borkowski et al., 1990). Dabei ist es wichtig, zwischen den Konstrukten Fähigkeitsselbstkonzept (kognitiv und eher deskriptiv) und den emotionalen Folgen dieser kognitiven Repräsentationen, dem Selbstwert (affektiv und eher evaluativ) zu differenzieren (vgl. Schöne, Dickhäuser, Spinath \& Stiensmeier-Pelster, 2003).

Das Konstrukt Selbstkonzept lässt sich theoretisch und empirisch nur vage charakterisieren. Über seine Struktur, Wirkungsweise und Entstehung existieren sehr verschiedene Modelle und Hypothesen. Während man früher von einem eindimensionalen, globalen Selbstkonzept ausging, besteht heute weitgehend Einigkeit, dass es sich hierbei um ein differenziertes, vielschichtiges und teilweise hierarchisch geordnetes System handelt. Ein sehr einflussreiches Modell zur Struktur des Selbstkonzeptes stammt von Shavelson, Hubner und Stanton (1976). Es differenziert zwischen akademischem Selbstkonzept und nichtakademischem Selbstkonzept mit jeweils weiteren Unterteilungen. Kontroverse Befunde führten zu einer Revision dieses Modells. Die überarbeitete Form von Marsh und Shavelson (1985) gliedert das akademische Selbstkonzept in zwei getrennte Subkonzepte: in das mathematisch orientierte und das verbale bzw. sprachliche Selbstkonzept, denen jeweils noch Faktoren zweiter Ordnung zugrunde liegen (Marsh, 1990). Inzwischen gibt es eine Reihe alternativer Modelle zu der kognitiven Organisation des Selbst-Systems (vgl. Kihlstrom, 1993; Srull \& Wyer, 1993).

Zur Erfassung verschiedener Aspekte des Selbstkonzeptes existieren unterschiedliche Skalen und Erhebungsinstrumente, meist Fragebögen. Der Zusammenhang von globalem Selbstkonzept und Leistung fällt mit $r=.21$ eher niedrig aus. Mit zunehmendem Alter und zunehmendem Fähigkeitsniveau steigt die Korrelation je nach Studie auf $r=.42$ (Krapp, 1997) bis $r=.70$ (Nicholls, 1979). Allerdings bleibt die Frage nach der Kausalrichtung und dem Zusammenhang zwischen Selbstkonzept und der Nutzung mnemotechnischer Strategien weitgehend unbeantwortet. Der self-enhancement Ansatz nimmt an, dass das Fähigkeitsselbstkonzept die Leistung einer Person beeinflusst, der skill-development Ansatz postuliert die entgegengesetzte Kausalrichtung. Verschiedene Längsschnittstudien stützen beide Sichtweisen (z.B. Helmke \& van Aken, 1995; Stiensmeier-Pelster, Balke \& Schlangen, 1996), so dass vermutlich beide Ansätze zutreffend sind. Ein allgemeines Modell, das einen Zusammenhang zwischen Strategieanwendung und Selbstkonzept formuliert, ist das Modell des good strategy user (Pressley, Borkowski \& Schneider, 1987, 1989). Das positive Selbstkonzept stellt in diesem allgemeinen Rahmenmodell neben breitem Strategierepertoire, gutem Selbstmanagement 
und einer Erfolgsattribution auf Anstrengung einen zentralen Faktor für erfolgreiches Lernen dar (vgl. McCormick \& Pressley, 1997).

Zahlreiche Studien haben gezeigt, dass Kinder bei der Einschulung ein sehr optimistisches Selbstkonzept aufweisen (z.B. McCombs, 1986). Die Höhe der Einschätzung eigener Fähigkeiten nimmt im Laufe der Grundschulzeit ab (Helmke, 1999), bleibt aber im Mittel im überdurchschnittlichen Bereich. Gleichzeitig wird das Selbstkonzept durch den Einfluss verschiedener Faktoren differenzierter, und der Zusammenhang zwischen dem Selbstbild eigener Leistung und entsprechenden externen Leistungsindikatoren (z.B. Schulnoten) steigt (vgl. Eccles, Roeser, Wigfield \& Freeman-Doan, 1999; Marsh, Ellis \& Craven, 2002). Eine Ursache für die Veränderungen sind soziale Vergleichsprozesse, die ab der mittleren Kindheit einsetzen (vgl. Frey \& Ruble, 1990; Ruble \& Frey, 1991). Nach Marsh, Byrne und Shavelson (1988) vergleichen Kinder dabei ihre Leistung in einem Bereich mit der von anderen Schülern in demselben Bereich (external frame) und mit der eigenen Leistung in einem anderen Bereich (internal frame). Diese Vergleichsprozesse führen zu einem Wandel von einem optimistischen zu einem realistischen und differenzierten Selbstkonzept eigener Fähigkeiten (Helmke, 1999). Der Verlauf der Selbstkonzepteinschätzung folgt dabei einem linearen Trend. Die starke Streuung der durchschnittlichen Verlaufsgestalt lässt dahinter jedoch verschiedene Verlaufsformen vermuten (vgl. Helmke, 1998, 1999).

Obwohl verschiedene Autoren motivationalen Komponenten (und damit auch dem Selbstkonzept eigener Fähigkeiten) einen Einfluss auf die kognitive Performanz zusprechen (z.B. Schneider, 2000), ist die genaue Rolle der Ausprägung und Veränderung des kindlichen Selbstkonzepts, für die Entwicklung und Anwendung von Gedächtnisstrategien - speziell Rehearsal - bislang weitgehend ungeklärt.

\subsection{Integrative Theorien der Strategieentwicklung}

Eine allgemeine Theorie der Entwicklung kognitiver Fähigkeiten und des Wissens sollte berücksichtigen, dass sich die entwickelnde Leistungsfähigkeit des Gedächtnisses nicht alleine durch die Zunahme strategischen Verhaltens erklären lässt und daher die dargestellten weiteren Gedächtnisdeterminanten integrieren. Ein Versuch, die entscheidenden Charakteristika des ausgereiften strategischen Verhaltens in ein allgemeines Rahmenmodell einzufügen, stammt von Folds et al. (1990). Sie weisen auf die oft starke Kontextvariabilität und -spezifität strategischen Verhaltens hin und fokussieren auf diesen Faktor, um die mnemotechnischen Fähigkeiten von Kindern zu charakterisieren. Dabei beziehen sie sich auf drei 
Dimensionen strategischen Verhaltens: die Zielgerichtetheit strategischer Aktivitäten, ihre Konsistenz (Generalisierbarkeit) und ihre Effektivität. Folds et al. (1990) benennen fünf Aufgabendimensionen, die das strategische Verhalten beeinflussen und die ihrer Ansicht nach eine präzisere Beschreibung kindlich-strategischer Fähigkeiten erlauben. Im Einzelnen sind dies das vorgegebene bzw. vom Kind angenommene Ziel der Aufgabe, die Art des Lernmaterials, der Einfluss der Instruktion auf den Strategiegebrauch, das Ausmaß an Informationsverarbeitungsanforderungen durch die Aufgabe und der Einfluss der Wissensbasis (Wissen über und Vertrautheit mit dem Material). Auf der Grundlage dieser Faktoren lässt sich die Entwicklung mnemotechnischer Kompetenzen als a) eine zunehmende Generalisierung strategischen Verhaltens und b) einen alterskorrelierten Leistungsanstieg beschrieben. Als weitere Faktoren, die einen Beitrag zur größeren Effektivität des Strategiegebrauchs älterer Kinder leisten, nennen Folds et al. (1990, S. 76) ,a) increases in metamnemonic understanding, b) experiences in school, c) the growth of the knowledge base, and d) the increased efficiency in strategy use“.

Hasselhorn $(1995,1996)$ stellt mit seiner Strategie-Emergenz-Theorie ein theoretisches Rahmenmodell zu den kognitiven Mechanismen weiterentwickelten strategischen Organisierens vor. Er berücksichtigt dabei verschiedene Determinanten des kategorialen Organisierens, die Wechselwirkungen zwischen diesen Faktoren und Wechselwirkungen zwischen internen Merkmalen und externen bzw. kontextuellen Bedingungen. Die Strategie-Emergenz-Theorie integriert das Modell des semantischen Netzwerkes (Rabinowitz \& Chi, 1987), das Arbeitsgedächtnis-Modell (Baddeley, 1986) und Tulvings Synergistic-Ecphory-Modell (Tulving, 1982, 1983). Hasselhorn (1995, 1996) nimmt die Möglichkeit einer strategischen Wissensaktivierung und der sich entwickelnden strategischen Nutzung eigenen kategorialen Wissens an. Damit geht er über frühere Positionen hinaus, die die Wissensaktivierung für einen rein automatisch ablaufenden Prozess halten (Bjorklund, 1985, 1987). Zusätzlich zu diesen Automatismen, die durch die funktionale Kapazität des Arbeitsgedächtnisses und verfügbare, kontextbezogene Wissensbestände aus der Wissensbasis determiniert sind, postuliert die Strategie-Emergenz-Theorie, „dass sich zwischen 8 und 10 Jahren infolge eines zunehmend angemessenen aufgabenspezifischen Metagedächtnisses auch strategische Möglichkeiten der kategorial geordneten Wissensaktivierung herausbilden“ (Hasselhorn, 1996, S. 83). Die qualitativen Veränderungen in den mnemotechnischen Kontrollprozessen (Gedächtnisstrategien), die im Verlauf der Grundschulzeit beobachtet werden, sind danach hauptsächlich die Folge der Zunahme an spezifischen Metakognitionen. Somit können Veränderungen der Wissensbasis zwar einen Teil der Zunahme kategorialen Organisierens in der Grundschule erklären, die 
weitaus größere Steigerung des strategischen Verhaltens zwischen 8 und 10 Jahren wird jedoch durch eine enge Metakognition-Kognition-Relation vermittelt.

Hasselhorn (1996, S. 77) geht davon aus, dass das erstmalige Auftreten von strategischen Organisationsprozessen zeitlich mit dem Übergang von basalen zu kumulativen Rehearsal-Techniken zusammenfällt. Beiden qualitativen Veränderungen wird derselbe Mechanismus zugrunde gelegt, der aus dem Erwerb metakognitiver Kompetenzen und einer Sensitivität für Situationen, in denen man dieses Wissen nutzen kann, besteht. Danach hat ein elaboriertes metakognitives Strategiewissen den Gebrauch effizienterer Verarbeitungsmechanismen in Form kumulativen Rehearsals zur Folge, welche durch die zentrale Exekutive initiiert werden. Die dadurch bedingte tiefere Verarbeitung des Lernmaterials führt zu einer stärkeren Aktivierung der Items im semantischen Netzwerk und zur Bildung von multiplen Abrufpfaden, was sich letztlich in einer besseren Lernleistung niederschlägt. Ein erster Versuch, die Annahmen der Strategie-Emergenz-Theorie auf Rehearsal-Prozesse zu übertragen und zu prüfen, ob diese für die Entwicklung der Strategie des kategorialen Organisierens bewährte Theorie auch die Herausbildung kumulativen Rehearsals beschreiben und erklären kann, wurde von Hasselhorn und Lindner-Müller (1995) unternommen. Die Ergebnisse ihrer Studie sprechen eher gegen diese Möglichkeit. Allerdings setzten die Autoren zur Überprüfung ihrer Vorhersagen kategorisierbares Lernmaterial ein, um auch die Konvergenz und die kombinierte Nutzung von kategorialem Organisieren und kumulativem Rehearsal zu erfassen. Bei dem gewählten Aufgabentyp erweist sich die kategoriale Sortierstrategie als deutlich nützlicher - Rehearsal-Prozesse treten dagegen eher in den Hintergrund (vgl. Ornstein, Naus \& Miller, 1977). Weiterhin ist die kombinierte Anwendung beider Strategien gerade bei jüngeren Kindern wegen der damit verbundenen hohen mentalen Anforderungen (noch) nicht zu erwarten (vgl. Guttentag, 1984). Die einseitige Beschränkung auf overte RehearsalAktivitäten kann darüber hinaus zu einer Unterschätzung kumulativen Rehearsals geführt haben. Die Autoren konnten jedoch zeigen, dass die kombinierte Nutzung von kumulativem Rehearsal und kategorialem Organisieren in Abhängigkeit vom deklarativen Metagedächtnis deutlich mit dem Alter anstieg. Insgesamt können aus der Strategie-Emergenz-Theorie für die Entstehung kumulativen Rehearsals vier empirische Vorhersagen abgeleitet werden (vgl. Hasselhorn, 1996; Hasselhorn \& Lindner-Müller, 1995), die zum Teil durch die Studie von Guttentag et al. (1987) gestützt werden. Danach ist zu erwarten, dass es

- zwischen 9 und 10 Jahren zu einem Anstieg metakognitiven Wissens über RehearsalProzese kommt. 
- zu einem parallelen Anstieg der Anzahl an Kindern kommt, die dieses strategische Verhalten bei der Bearbeitung von episodischen Gedächtnisaufgaben nutzen (vgl. Guttentag et al., 1987).

- bei älteren Kindern (jedoch nicht bei jüngeren) einen positiven Zusammenhang zwischen dem strategiespezifischen Metagedächtnis und entsprechendem Verhalten gibt.

- im Zusammenhang mit der Strategie-Emergenz auch zu besseren Behaltensleistungen (als Konsequenz der effektiveren Nutzung der Rehearsal-Strategien) kommt (vgl. Guttentag et al., 1987).

\subsection{Zusammenfassung}

Das Ziel der bisherigen Ausführungen war es, die Befunde zur Entwicklung mnemotechnischer Strategien, speziell der Rehearsal-Strategien, darzustellen. Des Weiteren sollten die Rahmenbedingungen zunehmender strategischer Kompetenz und Effizienz im Verlauf der Grundschuljahre erörtert werden. Insgesamt zeigen die bis jetzt durchgeführten Studien, dass es im Verlauf der Grundschuljahre zu qualitativen Veränderungen im Rehearsal-Stil kommt, die mit einem Anstieg der Lernleistung in diesem Zeitraum verbunden sind. Die aktiveren Memoriertechniken werden als eine entscheidende Determinante des alterskorrelierten Anstiegs der Wiedergabeleistung betrachtet. Zeitgleich kann auch eine Verbesserung der Kurzzeitgedächtnisleistung, eine wachsende Elaboriertheit der Wissensbasis und ein Anstieg an metakognitivem Strategiewissen verzeichnet werden. Darüber hinaus wird motivationalen Faktoren und dem Selbstbild eigener Fähigkeiten ein mediierender Einfluss auf die Entwicklung dieser Determinanten, speziell auf die zunehmende Nutzung strategischer Lernprozesse, attestiert.

Die von Ornstein und seiner Arbeitsgruppe durchgeführten experimentellen Untersuchungen legen einen graduellen bzw. sequenziellen qualitativen Wechsel von passiven zu aktiveren mnemotechnischen Strategien im Verlauf der Grundschuljahre nahe. Dieser Shift findet nicht plötzlich statt, sondern kann als eine Art Kontinuum verstanden werden. Bei jüngeren Kindern ist der Gebrauch von Rehearsal dabei stark von Kontextfaktoren abhängig. Die erstmalige Anwendung einer neuen Rehearsal-Strategie geschieht eher unter supportiven Rahmenbedingungen und ist noch wenig profitabel hinsichtlich des daraus resultierenden Lerngewinns. Vermehrte Übung und die damit verbundene Automatisierung führen schließlich zu einer zunehmenden Generalisierung, durch die die Strategie bei neuen Inhalten und in 
neuen Kontexten genutzt wird, und zu einer steigenden Effizienz in Form eines Leistungsanstiegs.

Die bisherigen Studien zur Entwicklung von Rehearsal-Strategien waren im Allgemeinen experimentelle Gedächtnisanalysen im Altersvergleich, bei denen Kinder unterschiedlicher Altersgruppen zum selben Zeitpunkt untersucht wurden. Da hierbei nicht das Individuum in seinen Veränderungen über die Zeit Untersuchungseinheit ist, liefern sie keine Informationen über die intraindividuellen Veränderungen. Auch die beiden kleineren längsschnittlich angelegten Studien zur Entwicklung von Rehearsal-Strategien sind in ihrer Aussagekraft aufgrund des großen zeitlichen Abstandes zwischen den Messzeitpunkten und der kleinen Stichprobengröße deutlich eingeschränkt. Dabei ist der Studie von Guttentag et al. (1987) mit einer Stichprobengröße von N=30 und einem Abstand von einem Jahr zwischen den Messzeitpunkten sicherlich eine höhere Güte zu bescheinigen als der Studie von Kunzinger (1985), die mit einem Zweijahresabstand und einer Gruppengröße von N=18 Probanden durchgeführt wurde. Es fehlen also bislang weitgehend Studien, die die querschnittlichen Befunde durch die Erfassung individueller Entwicklungsverläufe bestätigen. Ornstein et al. (1988, S. 47) fordern daher zu Recht: “...a complete understanding of the development of memory requires a longitudinal analysis of children at all stages of mnemonic competence".

Weiterhin gibt es bislang kein strategiespezifisches komplexes Funktionsmodell, das die Entwicklung der Rehearsal-Stragien und der oben dargestellten weiteren Gedächtnisdeterminanten in ein allgemeines und integratives Rahmenmodell zusammenfasst. Allerdings liegt mit der von Hasselhorn (1996) vorgestellten Strategie-Emergenz-Theorie ein Erklärungsansatz für die Entwicklungsveränderungen zwischen 8 und 10 Jahren bei der Gedächtnisstrategie des kategorialen Organisierens vor. Dieses Modell geht von der strategischen Nutzung metakognitiven Wissens ab dem Ende der Grundschulzeit aus. Dem elaborierten Strategiewissen wird dabei der entscheidende Einfluss auf die Qualität der Informationsverarbeitung und die Nutzung effizienterer Verarbeitungsmechanismen zugesprochen. Hasselhorn (1996; Hasselhorn \& Lindner-Müller, 1995) nimmt weiterhin an, dass die Entwicklung des kategorialen Organisierens und des kumulativen Rehearsals prinzipiell auf demselben Mechanismus beruhen - auf der Veränderung des strategiespezifischem Metagedächtniswissen und dessen Anwendung auf Lern- und Abrufprozesse bei episodischen Gedächtnisanforderungen. 


\section{Eigene Untersuchung und psychologische Hypothesen}

Kognitive Entwicklungsanalysen erfordern nach Ornstein (1999) das parallele Herangehen an drei unterschiedliche Forschungsaufgaben (vgl. auch Ornstein \& Haden, 2001):

- die Charakterisierung der Basiskompetenzen auf unterschiedlichen Altersstufen,

- die Erfassung alterskorrelierter Veränderungen dieser Kompetenzen,

- die Formulierung kausaler Einflussfaktoren für die entstehenden Entwicklungsmuster.

Diese Anforderungen werden von den vorliegenden Studien zur Entwicklung von RehearsalStrategien kaum erfüllt, da eine Querschnittsanalyse in der Regel nicht in der Lage ist, die zeitliche Abfolge von Effekten aufzuzeigen. Um das Ausmaß an intraindividueller Stabilität der Memorierprozesse und die Stabilität der interindividuellen Unterschiede in den Entwicklungsmustern erfassen zu können, sind längsschnittliche Analysen unabdingbar (vgl. Stemmler, Lösel \& Erzigkeit, 1998). Nur sie erlauben es, die subtilen Veränderungen in der Strategieentwicklung im Verlauf der Grundschuljahre aufzuspüren. In früheren Arbeiten konnte der Zusammenhang zwischen der Rehearsal-Set-Größe und der Reproduktionsleistung im Sinne einer Kovariation dieser beiden Faktoren aufgezeigt werden. Um jedoch zur Annahme eines Kausalzusammenhangs zu gelangen, ist eine entsprechende Theorie nötig, die auch die anderen Determinanten der Gedächtnisentwicklung berücksichtigt, da eine Kausalbeziehung nicht einfach aus einer beobachteten Kovariation zweier Faktoren erschlossen werden kann (Hager, 1992a). „Für die Prüfung von Kausalhypothesen ist ... die Konzeption eines kausalen rekursiven Systems [entscheidend]“ (Bredenkamp, 1983, S. 5). Die Dominanz von Querschnittstudien im Bereich der Gedächtnisentwicklung ist auch insofern bedauerlich, als nur Longitudinalstudien Rückschlüsse auf die Einflussrichtungen der weiteren Gedächtnisdeterminanten zulassen.

Hier scheint die Strategie-Emergenz-Theorie (Hasselhorn, 1996) einen guten Erklärungsrahmen für die qualitativen Veränderungen der Rehearsal-Strategien im Verlauf der späteren Grundschuljahre zu bieten: Die von Guttentag et al. (1987) berichtete Verlagerung von einfachen zu kumulativen Memorierprozessen zwischen 8 und 10 Jahren deckt sich mit den Annahmen der Strategie-Emergenz-Theorie. Danach nutzen Kinder mit etwa 10 Jahren erstmals ihr metakognitives Wissen spontan für bislang weitgehend unreflektiert ausgeführte Lernprozesse (vgl. Hasselhorn \& Grube, im Druck). Diese enge Metakognition-KognitionRelation führt zu einem vermehrten Gebrauch kumulativen Rehearsals und in ihrer Folge zu besseren Behaltensleistungen durch eine stärkere Aktivierung von Knoten im semantischen Netzwerk. Durch die Ausbildung multipler Abrufpfade wird somit eine Emergenz strategischer Prozesse erzielt. Auch das Selbsttestverhalten einiger älterer Kinder bzw. Jugendlicher 
in der Studie von Guttentag et al. (1987) deckt sich mit den Annahmen der StrategieEmergenz-Theorie, signalisiert es doch ausgeprägtes metakognitives Strategiewissen. Leider wurde dieses von den Autoren nicht näher erfasst.

Die längsschnittliche Analyse der Rehearsal-Prozesse, weiterer Determinanten der Gedächtnisentwicklung und der erbrachten Lernleistung sollte auch die Beantwortung der Frage ermöglichen, ob das ontogenetisch erstmalige Auftreten spontaner kumulativer Wiederholtechniken ohne einen entsprechenden Leistungsgewinn erfolgt. Für eine solche Nutzungsineffizienz bei Rehearsal-Strategien spricht bislang nur die Literaturdurchsicht von Miller und Seier (1994). Allerdings berichten diese Autoren auch Studien mit gegenläufiger Evidenz. Längsschnittliche Analysen der intraindividuellen Veränderungen im Strategiegebrauch des kategorialen Organisierens lassen an der generellen Gültigkeit bzw. Normativität eines Nutzungsdefizits in der Strategieentwicklung zweifeln (Schlagmüller \& Schneider, 2002; Sodian \& Schneider, 1999).

In den folgenden Kapiteln wird eine Untersuchung dargestellt, anhand derer eine Beantwortung der angedeuteten offenen Fragen ermöglicht werden soll. Es würde allerdings den Rahmen der vorliegenden Arbeit sprengen, wollte man alle in Abschnitt 2.3 aufgeführten Determinanten, ihren Zusammenhang zur Memorierstrategie und ihre Veränderung im Verlauf der späten Grundschuljahre berücksichtigen. Da hier viele, in den letzten 20 Jahre innerhalb der kognitiven Entwicklungspsychologie eher allgemein bzw. ausschließlich mit Bezug auf die Organisationsstrategie behandelte Themen, erstmals auf Rehearsal-Prozesse angewandt werden, sollen die oben angeführten zentralen Themen im Mittelpunkt der Analysen stehen. Hierfür wird zwischen verschiedenen Betrachtungsebenen unterschieden (vgl. Hager, 1992a, 1992b). Die Ebene der psychologischen Hypothesen (PH) enthält dabei Aussagen über den Zusammenhang von psychologischen Variablen, von denen auf der zweiten Betrachtungsebene psychologische Vorhersagen (PV) abgeleitet werden. Diese stellen eine Konkretisierung der angesprochenen Zusammenhänge im Rahmen des gewählten Versuchsplans dar. Aus den bisherigen Ausführungen lassen sich dabei drei allgemeine Zielsetzungen der vorliegenden Arbeit generieren.

Als erstes geht es darum, die zumeist querschnittlichen Befunde über die Entwicklung des kumulativen Rehearsals und den behaupteten kausalen Zusammenhang zur Reproduktion mittels Longitudinalanalysen kritisch zu beleuchten. Dabei erfolgt die Überprüfung anhand der aus der Literatur hervorgehenden Kriterien. Auf der deskriptiven Ebene wäre danach eine alterskorrelierte Zunahme in der Qualität des Memorierverhaltens und der Gedächtnisleistung sowie ein mit dem Alter steigender Zusammenhang zwischen diesen beiden Faktoren 
zu erwarten $\left(P H_{l}\right)$. Darüber hinaus sollten sich bei längsschnittlichen Betrachtungen hohe zeitliche Stabilitäten bezüglich des Memorierverhaltens und der Gedächtnissleistung zeigen $\left(\mathrm{PH}_{2}\right)$. Eine offene Frage ist weiterhin, ob Anzeichen für Memorierprozesse zu Beginn der Abrufphase gefunden werden können bzw. ob durch Rehearsal-Prozesse Abrufphänomene hervorgerufen werden, die sich z.B. in Form von kürzeren Latenzzeiten oder Organisationsprozessen in der Output-Phase zeigen (vgl. Abschnitt 2.1.3).

Als zweites soll der Frage nachgegangen werden, ob die Strategie-Emergenz-Theorie (Hasselhorn, 1996) als Erklärungsmodell für das Auftreten von kumulativem Rehearsal und den dieser Entwicklung zugrunde liegenden Prozessen angemessen ist. Folgt man der Annahme der Entwicklungsparallelität von kategorialem Organisieren und Rehearsal, wie sie von Hasselhorn und Lindner-Müller (1995) formuliert wurde, so sollte es im Alter zwischen 9 und 10 Jahren zu einem deutlichen Anstieg an metakognitivem Wissen über den Nutzen und die Einsatzmöglichkeiten kumulativer Rehearsal-Prozesse und zeitgleich zu ihrer verstärkten Verwendung bei der Bearbeitung von episodischen Gedächtnisaufgaben kommen. Bei älteren Kindern sollte sich ein deutlicher Zusammenhang zwischen diesen beiden Faktoren und ebenfalls ein Leistungsgewinn aus der Anwendung des kumulativen Rehearsals finden lassen (PH3). Dagegen sollte der Einfluss kapazitiver Aspekte auf die Gedächtnisleistung im Verlauf der späten Grundschuljahre zurückgehen $\left(\mathrm{PH}_{4}\right)$.

Die dritte Zielsetzung der vorliegenden Untersuchung ist die Klärung der Frage, ob die erste spontane Anwendung kumulativen Rehearsals von einer Nutzungsineffizienz begleitet ist. Dies würde sich darin zeigen, dass der erste spontane Gebrauch kumulativer RehearsalProzesse bzw. die hauptsächliche Nutzung kumulativen Rehearsals zumindest von keiner korrespondierenden Leistungssteigerung, vielleicht sogar von einem Leistungsrückgang begleitet wäre. Auch wenn neu als Strategen klassifizierte Kinder keine bedeutsamen Steigerungen der Reproduktionsleistung zwischen zwei Messzeitpunkten zeigen, kann dies als Hinweis auf eine Nutzungsineffizienz für Rehearsal interpretiert werden $\left(\mathrm{PH}_{5}\right)$. 


\section{Methode}

\subsection{Untersuchungsdesign und Auswahl der Stichprobe}

In der vorliegenden Studie ${ }^{1}$ wurde ein kombiniertes Querschnitt-Längsschnitt-Design realisiert. Die längssschnittliche Betrachtung erfolgte von Ende der zweiten bis Anfang der vierten Klasse. Die Kinder der Longitudinalgruppe bearbeiteten zu vier Messzeitpunkten in je halbjährigem Abstand verschiedene Lernaufgaben.

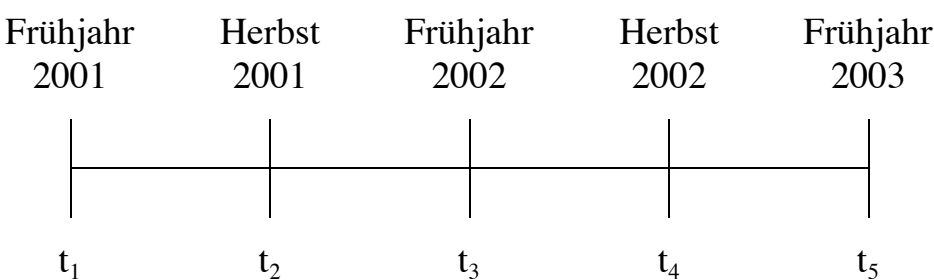

Messzeitpunkt

2 .

3.

3.

4.

$\begin{array}{lll}\text { Klassenstufen im Querschnitt 4., } 6 . & \text { 2., } 4 ., 6 .\end{array}$

Abbildung 4.1: Untersuchungsplan der vorliegenden Studie

Gegenüber früheren Längsschnittstudien ist somit, wie von Ornstein (1999) gefordert, die Dichte bzw. Frequenz der Messzeitpunkte erhöht. Um einen Vergleich zwischen Längsund Querschnittbefunden zu ermöglichen und den Einfluss von Übungseffekten abschätzen zu können, wurden zu verschiedenen Messzeitpunkten zusätzliche Querschnitt-Kontrollstudien durchgeführt. Abbildung 4.1 veranschaulicht den Untersuchungsplan.

Für die Gewinnung der Stichprobe wurden in Göttingen und Umgebung Schülerinnen und Schüler der zweiten, vierten und sechsten Klassen und deren Erziehungsberechtigte über die Studie informiert und um Unterstützung gebeten. Voraussetzung für die Teilnahme war das Vorliegen einer schriftlichen Einverständniserklärung des/der Erziehungsberechtigten. Um trotz möglicher Dropouts in der Longitudinalgruppe eine komfortable Teststärke für die geplanten Analysen und eine hohe Repräsentativität der Ergebnisse zu erzielen, wurde die Stichprobengröße dieser Gruppe etwas höher angesetzt als die Größe der Vergleichsgruppen.

\footnotetext{
${ }^{1}$ Die vorliegende Untersuchung ist Bestandteil einer Studie zu den Bedingungen und intraindividuellen Entwicklungsverläufen strategischer Gedächtnisprozesse zwischen 5 und 12 Jahren. Das von der Deutschen Forschungsgemeinschaft (DFG) finanzierte Projekt stellt eine Kombination zweier, sich im Sinne eines Sequenzplans ergänzenden Längsschnittstudien in Göttingen und Würzburg dar.
} 


\subsection{Beschreibung der Stichprobe}

Tabelle 4.1: Probandenverteilung in der Longitudinalgruppe: Alters- (Mittelwerte und Streuungen) und Geschlechterverteilung.

\begin{tabular}{|c|c|c|c|c|}
\hline Messzeitpunkt (t) & $\mathrm{t}_{1}$ & $\mathrm{t}_{2}$ & $\mathrm{t}_{3}$ & $\mathrm{t}_{4}$ \\
\hline Klassenstufe & 2. & 3. & 3. & 4. \\
\hline Alter (Jahre; Monate) & $8 ; 3(5.5)$ & $8 ; 8(5.7)$ & $9.1(5.5)$ & $9 ; 7(5.5)$ \\
\hline Geschlecht (w/m) & $50 / 30$ & $50 / 30$ & $50 / 30$ & $50 / 30$ \\
\hline
\end{tabular}

Die Studie begann im Mai 2001 mit 111 Zweitklässlern aus 15 Schulen der Landkreise Göttingen und Einbeck. In die vorliegende Untersuchung fließen davon die Daten der 80 Kinder mit vollständigen Datensätzen vom ersten bis zum vierten Messzeitpunkt ein. Das Durchschnittsalter in dieser Gruppe (50 Mädchen, 30 Jungen) betrug beim ersten Messzeitpunkt 8;3 Jahre (Tabelle 4.1).

Tabelle 4.2: Probandenverteilung in den Querschnittgruppen: Alters- (Mittelwerte und Streuungen) und Geschlechterverteilung, getrennt nach Messzeitpunkten und Klassenstufen.

\begin{tabular}{|c|c|c|c|c|c|}
\hline Messzeitpunkt (t) & $\mathrm{t}_{1}$ & $\mathrm{t}_{1}$ & $\mathrm{t}_{5}$ & $\mathrm{t}_{5}$ & $\mathrm{t}_{5}$ \\
\hline Klassenstufe & 4. & 6. & 2. & 4. & 6. \\
\hline Alter (Jahre; Monate) & $10 ; 5(6.1)$ & $12 ; 2(5.0)$ & $7 ; 11(6.1)$ & $10 ; 3(6.5)$ & $12 ; 0(3.9)$ \\
\hline Geschlecht (w/m) & $8 / 12$ & $13 / 7$ & $12 / 8$ & $12 / 8$ & $9 / 11$ \\
\hline
\end{tabular}

Das durchschnittliche Alter der Kinder in der Zweitklässler-Querschnittstichprobe ( $\mathrm{n}=20 ; 12$ Mädchen, 8 Jungen) war 7;11 Jahre, das der Viertklässler ( $\mathrm{n}=40 ; 20$ Mädchen, 20 Jungen) 10;4 Jahre und das der Sechstklässler (n=40; 22 Mädchen, 18 Jungen) 12;1 Jahre (Tabelle 4.2). Nachfolgende Analysen zeigten, dass die Probanden der ZweitklässlerQuerschnittstichprobe durchschnittlich deutlich jünger als die der Längsschnittgruppe beim ersten Messzeitpunkt waren $(t(98)=36 ; p<.05)$. 


\subsection{Verwendete Verfahren und abhängige Variablen}

\subsubsection{Gedächtnis-Aufgabe}

Aufgabe: Zur Erfassung von Rehearsal-Prozessen wurde eine serielle Lernaufgabe eingesetzt, die sich an dem von Guttentag et al. (1987) berichteten Verfahren orientierte. Das Lernmaterial bestand aus drei Sets mit je 14 konkreten Substantiven ohne kategoriale Zusammenhänge innerhalb der einzelnen Sets. Jedes Stimuluswort und ein Bild des betreffenden Items wurden auf eine weiße 5 x $5 \mathrm{~cm}$ große Indexkarte gedruckt. Darüber hinaus erfolgte eine Aufnahme der Lernlisten auf CD mit einer Präsentationsrate von acht Sekunden (d.h. alle acht Sekunden ein Item). In gleicher Form wurden zwei Übungslisten mit je sechs Items erstellt. Ab dem zweiten Messzeitpunkt wurden die Lernlisten auf 12 Items gekürzt, und eine der beiden Übungslisten entfiel. Die für die Darbietung verwendeten Worte sind in Anhang A aufgelistet.

Die gewählte multimodale (audiovisuelle) Präsentationsform ermöglicht die parallele Verarbeitung im verbal-phonologischen und visuell-räumlichen Hilfssystem des Arbeitsgedächtnisses (s.o. Abschn. 2.3.1). Dies führt zu einer Reduktion der mentalen Belastung, wodurch mehr Aufmerksamkeitsressourcen für die Informationsverarbeitung oder andere (strategische) Prozesse zur Verfügung stehen (vgl. Brünken, Plass \& Leutner, 2003; Moreno \& Mayer, 2000). Auch sollten auf diese Weise Modalitätseffekte, die durch die Beschränkung auf die akustische oder visuelle Darbietungsform entstehen können, vermieden werden (vgl. Ornstein, Naus \& Stone, 1977).

Durchführung: Die Präsentationsrate von acht Sekunden wurde gewählt, um älteren Kindern hinreichend Gelegenheit für kumulatives Rehearsal zu geben, ohne bei jüngeren Kindern, die das Interstimulusintervall (noch) nicht vollständig durch Lernaktivitäten füllen, Aufmerksamkeitseinbußen und Motivationverluste herbeizuführen. Die Darbietungsfrequenz liegt zwischen den von Guttentag et al. (1987) realisierten 5 bzw. 10 Sekunden.

Auswertung: In die Auswertung gingen auf der Lern- und Reproduktionsseite verschiedene Maße als Abhängige Variablen ein: Zuerst wurde deskriptiv erfasst, welche Gedächtnisstrategie(n) ein Kind genutzt hatte. Anschließend wurde der prozentuale Anteil jeder Strategie an der möglichen Gesamtlernaktivität erfasst. Hatte eine Mnemotechnik einen Anteil von $\geq 50$ Prozent an der gesamten Lernaktivität, so stellte sie eine Haupt- oder Primärstrategie für das jeweilige Kind dar. Es gab folgende Hauptstrategien:

- einmalige Objektbenennung (Labeling). Beispiel: „Reifen“,

- mehrfache Wiederholung eines Items (singuläres Rehearsal). Beispiel: „Reifen, Rei- 
fen, Reifen“,

- Verknüpfung mehrerer Items in einer Wiederholungseinheit (kumulatives Rehearsal). Beispiel: „Reifen, Buch, Gabel, Reifen, Buch, Gabel““,

- semantische Verbindung mehrerer Items bzw. von einem Item mit Informationen im Gedächtnis (Assoziation-Elaboration). Beispiel: „Meine Mutter hat neue Reifen an ihrem Auto.“.

- Kinder, die keine der genannten Strategien mit einem Anteil von mindestens 50 Prozent verwendet hatten, wurden einer Restkategorie zugeordnet.

Zentrales Maß für das strategische Verhalten in der Enkodierphase stellte die mittlere Rehearsal-Set-Größe dar, also die durchschnittliche Anzahl unterschiedlicher Items, die ein Kind während der Interstimulusintervalle memorierte. Kumulative Memorierprozesse sind per Definition erst ab der Präsentation des zweiten Items durchführbar. Um hier durchgängig die Möglichkeit zu kumulativem Rehearsal zu geben, erfolgte ähnlich wie bei Guttentag et al. (1987) die Erfassung der memorierten Wörter erst ab dem dritten Interstimulusintervall. Die durchschnittliche Set-Größe diente ferner zur Bildung eines Kategorisierungssystems, bei dem drei qualitative Stufen strategischer Performanz unterschieden wurden:

- Bei einem mittleren Rehearsal-Set $\leq 1.25$ Items wurde ein Kind der Gruppe der „Noncumulative"-Rehearser zugeordnet.

- Bei einem mittleren Rehearsal-Set zwischen 1.25 und 2.25 Items wurde ein Kind der Gruppe der „Inconsistent“-Rehearser zugeordnet.

- Bei einem mittleren Rehearsal-Set $\geq 2.25$ Items wurde ein Kind der Gruppe der „Dominant-cumulative“-Rehearser zugeordnet.

Das Ausmaß an Beobachterübereinstimmung bei der Bewertung dieses strategischen Verhaltens bzw. der Exaktheit der vorgenommenen Auswertung wurde über die Interraterreliabilität bestimmt. Der Summenscore der durchschnittlichen Setgröße zeigte mit $r_{\mathrm{IR}}=.96(p<.01)$ eine sehr befriedigende Interraterreliabilität.

Die Summe der korrekt genannten Items (ohne Mehrfachnennungen oder Intrusionen, d.h. fälschlich erinnerte Items) diente als zentrale Abhängige Variable für die Reproduktionsleistung.

Analog zum Vorgehen von Naus et al. (1977) wurde die mittlere serielle Positionskurve über die drei Lernlisten so erstellt, dass zwei aufeinander folgende Items je eine Position der Kurve bildeten. Diese Darstellungsform ermöglicht eine bessere Vergleichbarkeit der Verläufe mit den Illustrationen der Arbeitsgruppe um Ornstein. 
Eine weitere Abhängige Variable stellte ein Maß dafür dar, inwieweit Kinder die serielle Präsentationsabfolge als Abrufstrategie genutzt hatten. Wie bereits von Hasselhorn et al. (submitted) wurde hierfür Tulvings (1962) Maß für subjektive Organisation (SO) variiert. In seiner ursprünglichen Form ist SO als $b r /(c+1)$ definiert, wobei $b r$ die beobachteten paarweise bidirektionalen Reproduktionen und $c$ die Anzahl gemeinsamer Items in zwei sukzessiven Recall-Trials bzw. Abrufsets darstellt. In der vorliegenden Untersuchung steht $b r$ für die Anzahl bidirektionaler Korrespondenzen zwischen Präsentations- und Reproduktionssequenz und $c$ für die Anzahl reproduzierter Items. SO kann Werte zwischen 0 und 1 annehmen, wobei höhere Werte für ein höheres Maß an subjektiver Organisation stehen. SO hat einen $\mathrm{Zu}$ fallswert von 0. Als letzte Abhängige Variable wurden die Inter-Response-Zeiten (Latenzen) analysiert und hinsichtlich verschiedener Kriterien ausgewertet.

\subsubsection{Zahlenspanne}

Aufgabe: Als Maß für die Kapazität des Kurzzeit- bzw. Arbeitsgedächtnisses wurde die Zahlenspanne vorwärts und rückwärts erhoben. Wie beim Subtest „Zahlennachsprechen vorwärts und rückwärts“ des HAWIK-III (Tewes, Rossmann \& Schallenberger, 2000) bestand die Aufgabe in der unmittelbaren Wiedergabe einer Serie von Zahlenabfolgen ansteigender Länge, die in vorgegebener oder umgekehrter Reihenfolge zu reproduzieren waren. Jede Unterserie der beiden Teile bestand dabei aus zwei Trials, d.h. aus zwei Ziffernfolgen gleicher Länge. Das Untersuchungsmaterial ist in Anhang C dargestellt.

Durchführung: Die akustische Präsentation der Sequenzen erfolgte von CD mit einer Rate von einer Ziffer pro Sekunde. Anders als im HAWIK-III wurden nur einsilbige Zahlen verwendet und daher die „7“ durch die „0“ ersetzt.

Auswertung: Ebenfalls entgegen den Vorgaben des HAWIK-III (Tewes, Rossmann \& Schallenberger, 2000) wurde hier als Maß für die Gedächtnisleistung die Zahlenreihe gewertet, die ein Kind als letztes korrekt wiedergeben konnte. Die Anzahl der Ziffern dieser Reihe ergab den Wert für die Gedächtniskapazität. Ihm wurden 0,5 Punkte hinzuaddiert, wenn ein Kind bei beiden Durchgängen der letzten Zahlenreihe erfolgreich war (vgl. Case et al., 1982; Hasselhorn, 1988).

\subsubsection{Metakognitives Strategiewissen}

Aufgabe: Die Erfassung von metakognitivem Strategiewissen erfolgte durch die Kombination zweier separater Methoden: zum einmal in Form von retrospektiven Selbstberichten 
der Kinder über Art und Nutzen der von ihnen angewandten Strategien bei der seriellen Lernaufgabe (metamemoriale Bewusstheit, Anhang B) und zum anderen über eine Skala zum strategiespezifischen deklarativen Metagedächtnis für Rehearsal. Diese bestand aus drei Aufgaben, die ab dem dritten Messzeitpunkt in eine Kurzversion der Würzburger Testbatterie zum deklarativen Metagedächtnis (Schlagmüller et al., 2001; Schneider, Schlagmüller \& Visé, 1998) eingebettet wurden (Anhang D).

Durchführung: Gefordert war die Bewertung unterschiedlicher Enkodierstrategien (Labeling, singuläres und kumulatives Rehearsal, Assoziation) zum Lernen von a) Sehenswürdigkeiten, b) Tieren und c) Orten. Für jede Frage wurden drei Antwortmöglichkeiten vorgegeben, die das Kind so mit Noten von 1 bis 5 bewerten sollte, dass einem guten Vorschlag eine gute Note, einem schlechten Vorschlag eine schlechte Note und einem mittelguten Vorschlag eine mittlere Note zugewiesen wurde. Dabei durfte eine Note auch mehrfach vergeben werden.

Auswertung: Die Auswertung erfolgte mittels Paarvergleichen. Dafür wurden die vorgeschlagenen Strategien in eine Rangordnung gebracht, mit kumulativem Rehearsal als bester, singulärem Rehearsal als zweitbester und Labeling bzw. Assoziation als schlechtester Enkodierstrategie. Für eine korrekte Antwort erhielt ein Kind einen Punkt. Für die Gleichbewertung zweier Antworten oder wenn es der besseren Antwortalternative die schlechtere Note gegeben hatte, bekam es dagegen keinen Punkt. Die Summe der Punktwerte bildet den Metagedächtnisscore. Die maximal erreichbare Punktzahl lag bei neun Punkten. Wie die Überprüfung der internen Konsistenz zeigte, weist die Skala mit Cronbachs-Alpha um .60 bei den Messzeitpunkten 3 und 4 eine noch ausreichende Reliabiltät auf $\left(\alpha_{3}=.67, \alpha_{4}=.59\right)$.

Das strategiespezifische Metagedächtnis für Rehearsal-Prozesse wurde erst ab dem dritten Messzeitpunkt erhoben. Dadurch sollte verhindert werden, dass in einer frühen Phase des Projektes eine komplexere Rehearsal-Strategie durch die Vorgabe einer solchen Lernmöglichkeit bei Kindern induziert wird, die das kumulative Rehearsal noch nicht für sich entdeckt haben. Mit dem dritten Messzeitpunkt traten die Kinder mehrheitlich in den Altersbereich ein, der früheren Studien (z.B. Guttentag et al., 1987) und den Annahmen der StrategieEmergenz-Theorie (Hasselhorn, 1996) zufolge den „kritischen Bereich“ der Strategieentwicklung markiert. Zeitgleich wurde eine Nachbefragung im Anschluss an die Bearbeitung der Free-Recall-Aufgabe eingeführt, mit der die metamemoriale Bewusstheit für Memoriertechniken erfasst wurde. Durch zwei Fragen sollte erhoben werden, wie sich die Kinder ihrer Meinung nach so viele Items hatten merken können (Bewusstheit für die in der Lernphase angewandte Strategie) und was das Gute an diesem Vorgehen ist (Bewusstheit für den Nutzen 
des strategischen Verhaltens für die Lernleistung). Die retrospektiven Selbstberichte der Kinder erlaubten auch eine Überprüfung und Validierung der beobachteten Strategien und damit der Verlässlichkeit der realisierten Overt-Rehearsal-Technik.

\subsection{Exkurs: Videoanalysen}

Ein zentraler Aspekt der vorliegenden Untersuchung ist die Analyse des strategischen Verhaltens von Kindern bei der Bearbeitung einer seriellen Lernaufgabe. Um die Annahmen über die Strategienutzung systematisch überprüfen zu können, wurden Videoaufnahmen des Lern- und Reproduktionsverhaltens der Kinder angefertigt. Der wissenschaftliche Wert der Videoanalysen für die Forschung und Theoriebildung liegt in der Möglichkeit, die Komplexität des (strategischen) Verhaltens bei der Bearbeitung einer Lernanforderung besser erfassbar zu machen und wiederholte Kodierungen bzw. Sekundäranalysen zu ermöglichen. Die Videodaten sind somit weniger theoriegebunden als andere Methoden der Datenerhebung. Sie erlauben die Generierung (neuer) analytischer Kategorien und damit die Einnahme unterschiedlicher Theorie- und Wahrnehmungsperspektiven. Nicht zuletzt sind durch die Präsentation von Videobeispielen wichtige Phasen der Strategieakquisition und -anwendung sowie deren Auswertung illustrierbar.

Während in früheren Arbeiten zur Entwicklung von Memorierstrategien häufig parallel zur Aufgabendurchführung eine reduktive Deskription des Lernverhaltens durch einen oder mehrere Beobachter stattfand (z.B. Flavell et al., 1966), erfolgte in der vorliegenden Untersuchung eine vollständige Erfassung des beobachtbaren Verhaltens durch Videoaufzeichnungen. Die Mikroanalyse des verbalen und nonverbalen Verhaltens erlaubte eine detaillierte Auswertung der strategischen Prozesse und ihrer Entwicklung. Besonders die Auswertung der Rehearsal-Protokolle in der Lernphase und der Latenzen in der Abrufphase wären ohne Videoanalysen nicht möglich gewesen.

In einem ersten Schritt wurden alle verbalen Äußerungen während der Lernphase protokolliert. Hier standen besonders die Itemnennungen in den Interstimulusintervallen im Fokus der Analysen. Durch die Auswertung der so gewonnenen Rehearsal-Protokolle konnte ein differenziertes Bild über die Quantität, Qualität (d.h. im Sinne Ornsteins die Anzahl unterschiedlicher Items, die in einem Interstimulusintervall memoriert wurden) und Konsistenz des verbalen strategischen Verhaltens gewonnen werden.

Mit der Erfassung nonverbaler Verhaltensaspekte sollte weiterhin überprüft werden, ob Gestik und Mimik Hinweise auf Kontrollstrategien geben bzw. Indikatoren für ein strategie- 
spezifisches Metagedächtnis für Rehearsal sind. Kontrollstrategien wie z.B. das Schließen der Augen beim Memorieren (vgl. Guttentag et al., 1987) oder das Mitzählen mit den Fingern während der Itempräsentation sowie während der Nennung der aktuell memorierten Items können als Indiz für Selbsttestverhalten vor dem Hintergrund eines ausgeprägteren metagkogntiven Strategiewissens interpretiert werden. Da jedoch erste Analysen keine Belege für solche Zusammenhänge oder überhaupt eine Systematik im nonverbalen Verhalten erbrachten, wurden sie bei späteren Messzeitpunkten nicht weiter verfolgt.

Auch die Aufnahmen der Reproduktionsphasen wurden anhand verschiedener Kriterien ausgewertet. Die zentrale Abhängige Variable war hier die Anzahl der reproduzierten Items. Darüber hinaus wurde auch die Reihenfolge der genannten Items inklusive Wiederholungen und Intrusionen anhand der Bandaufnahme protokolliert.

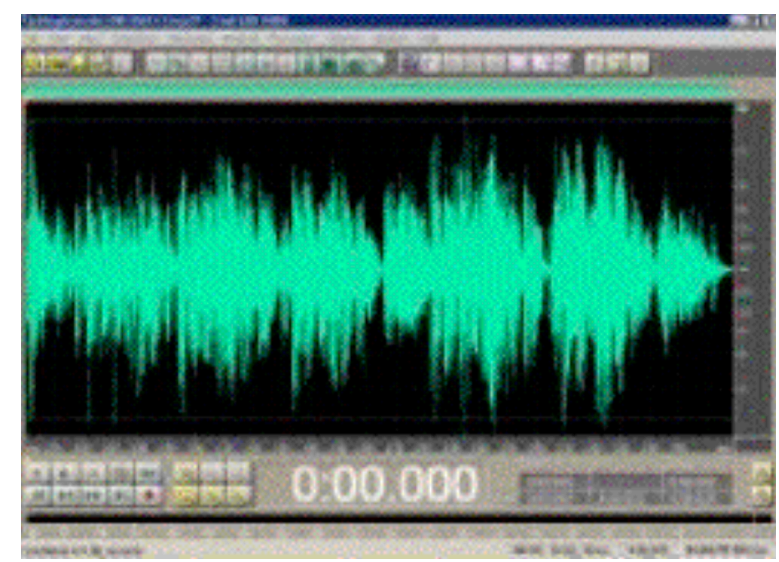

Abbildung 4.2: Beispiel für die spektrale Darstellung einer Tonspur

Die erstmals in dieser Form durchgeführte Auswertung der Latenzzeiten in der Reproduktionsphase einer Rehearsal-Aufgabe erforderte die Extraktion der Tonspur jeder Abrufphase von dem Videoband und ihre Weiterverarbeitung mit einem Audioschnittprogramm (Cooledit). Hierfür wurden in einem zweiten Schritt die Pausen zwischen der Nennung zweier Items in der spektralen Darstellung des Audiomaterials (Abbildung 4.2) markiert und ihre Dauer gemessen.

\subsection{Untersuchungsablauf}

Alle Untersuchungen fanden in den Räumen der Abteilung für Pädagogische Psychologie und Entwicklungspsychologie des Georg-Elias-Müller-Instituts für Psychologie der Universität Göttingen statt. Zu den Terminen wurden die Kinder in der Regel von den Erzie- 
hungsberechtigten gebracht. Kinder der Longitudinalgruppe kamen bei späteren Messzeitpunkten teilweise auch ohne ihre Eltern. Alle Testungen waren Einzeluntersuchungen. Sie wurden in den Nachmittagsstunden durchgeführt, um den Schulunterricht nicht zu beeinträchtigen. Ausnahmen stellten die Wochen der Oster- und Herbstferien dar, in denen auch Vormittagstermine vergeben wurden. Den Kindern wurden bei der Untersuchung verschiedene Gedächtnisanforderungen zur Bearbeitung vorgelegt.

Die erste Anforderung bestand in der Bearbeitung einer seriellen Lernaufgabe zur Erfassung von Wiederholstrategien. Die Kinder wurden von dem jeweiligen Versuchsleiter informiert, dass dieser gleich nacheinander einige Bildkarten auf den Tisch legen würde und dass man gleichzeitig von einem CD-Player höre, was auf den Karten abgebildet sei. Die Probanden wurden gebeten, gut zuzuhören und die Bilder ganz genau anzuschauen, da sie sich möglichst viele davon merken sollten. Weiterhin wurden die Kinder instruiert, nicht leise für sich, sondern laut sprechend zu lernen. Nachdem sich der Versuchsleiter versichert hatte, dass ein Kind die Instruktion verstanden und keine Fragen hatte, wurde das Vorgehen und die Methode des lauten Denkens anhand einer Liste mit sechs Items geübt: Der Versuchsleiter schaltete dazu den CD-Player an, über den die Items seriell im zeitlichen Abstand von acht Sekunden präsentiert wurden. Parallel zur Nennung eines Items wurde die korrespondierende Bildkarte so auf den Tisch gelegt, dass sie die vorherigen Bildkarten verdeckte. Nach der Präsentation aller Items des Übungssets wurde der CD-Player ausgeschaltet, der entstandene Kartenstapel entfernt und den Kindern eine Rückmeldung gegeben. Wenn ein Kind bereits laut sprechend gelernt hatte, wurde ihm gesagt, dass es sich bei den kommenden Durchgängen ebenso verhalten solle. Wenn es nicht oder nur selten laut gelernt hatte, wurde es noch einmal zum lauten Lernen aufgefordert. Danach erfolgte die freie Reproduktion der Liste. Beim ersten Messzeitpunkt schloss sich eine zweite Übungsliste mit gleicher Vorgehensweise an. Anschließend wurden die drei Lerntrials mit je 12 (beim ersten Messzeitpunkt 14) Items in der beschriebenen Form durchgeführt. Es handelt sich dementsprechend um eine SerialLearning-Free-Recall-Aufgabe. Die Kinder erhielten bei den Lernlisten keine weitere explizite Aufforderung zum lauten Lernen, selbst dann nicht, wenn ein Kind hier kein overtes Rehearsal (mehr) zeigte. Der Versuchsleiter protokollierte die jeweils genannten Items in der Reihenfolge ihrer Nennung. Ab dem dritten Messzeitpunkt folgte nach dem dritten Trial der Reproduktionsphase die Nachbefragung über die Art und den Nutzen der eingesetzten Lernstrategie(n). Die Bearbeitungsdauer der seriellen Lernaufgabe lag bei etwa 15 Minuten.

Die zweite Aufgabe war die Erfassung der Zahlenspanne. Die Durchführung erfolgte dabei wie im HAWIK-III (Tewes, Rossmann \& Schallenberger, 2000) vorgesehen: Die Kin- 
der wurden instruiert, dass sie ein paar Zahlen hören würden, die sie alle in korrekter Reihenfolge wiedergeben sollten. Das Vorgehen wurde anhand einer Zweiersequenz geübt. Dann wurden die Zahlensequenzen der Reihe nach von CD präsentiert. Nach jeder Sequenz machte der Versuchsleiter eine Pause, in der die Kinder die Zahlenreihe wiedergeben sollten. Für jede Aufgabe wurden beide Sequenzen einer Länge vorgegeben, auch wenn ein Kind bereits im ersten Versuch erfolgreich war. Die Aufgabe wurde abgebrochen, wenn ein Kind zwei Zahlenreihen gleicher Länge nicht oder falsch wiedergegeben hatte. Im Anschluss erfolgte die Durchführung der Zahlenspanne rückwärts in gleicher Weise.

Vor der Bearbeitung des Metagedächtnisfragebogens erklärte der Versuchsleiter, dass er nun einige Fragen vorlesen würde, bei denen verschiedene Lösungsvorschläge so beurteilt bzw. bewertet werden sollen, dass ein guter Vorschlag eine gute Note bzw. einen guten Platz, ein schlechter Vorschlag eine schlechte Note bzw. einen schlechten Platz und ein mittelguter Vorschlag eine mittlere Note bzw. einen mittleren Platz erhielten. Dabei könnte eine Note/ein Platz auch mehrfach vergeben werden. Die Fragen wurden einzeln vom Versuchsleiter laut vorgelesen und die Antworten der Kinder notiert. Ältere Kinder trugen die Antworten oftmals selber in den Fragebogen ein. Bei einigen Items sollte darüber hinaus eine Begründung für die gewählten Noten/Plätze angeben werden. Die Dauer des Interviews betrug etwa 15 Minuten.

\section{Ergebnisse}

Um eine bessere Übersichtlichkeit zu gewährleisten, ist der Ergebnisteil in fünf Abschnitte eingeteilt. In den ersten beiden Passagen wird eine Replikation der wesentlichen Befunde von Kunzinger (1985) und der Arbeitsgruppe um Ornstein (z.B. Guttentag et al., 1987; Ornstein \& Naus, 1978) versucht, indem a) ein querschnittlicher Vergleich der zweiten, vierten und sechsten Klassenstufen und b) eine längsschnittliche Entwicklungsanalyse auf Gruppenebene durchgeführt wurde. Im darauf folgenden Abschnitt stehen die intraindividuellen Entwicklungsverläufe im Fokus der Betrachtung. Durch den Einbezug von Maßen für die subjektive Organisation und die Itemlatenz werden hier auch vertiefende Analysen über Abrufphänomene dargestellt. Im vierten Ergebnisabschnitt erfolgt eine Überprüfung der Vorhersagen der Strategie-Emergenz-Theorie (Hasselhorn, 1996) für die Entwicklung kumulativer Memorierprozesse. Berichtet werden Zusammenhangsanalysen zwischen der Reproduktionsleistung und der Rehearsal-Set-Größe mit Maßen für die Gedächtniskapazität sowie dem strategiespezifischen Metagedächtnis als möglicher Determinanten von Rehearsal-Prozessen und Reproduktionsleistungen in den späten Grundschuljahren. Im letzten Abschnitt erfolgt die 
Beantwortung der Frage, ob das erste spontane Auftreten von kumulativem Rehearsal von einer Nutzungsineffizienz begleitet wird. Hierzu wird eine Klassifikation von Strategen und Nichtstrategen sowie Strategieentdeckern und -verlierern auf Individualebene gebildet. Zu Beginn der Ergebnisabschnitte sind die jeweiligen Vorhersagen und Hypothesen aufgeführt.

Für die folgenden Darstellungen und Berechnungen wurden die Lerndurchgänge eines Messzeitpunktes zusammengefasst und gemittelt. Hinsichtlich der Reproduktionsleistung zeigten sich mit einer Ausnahme keine Unterschiede der Wiedergabeleistung in den drei Lerntrials eines Messzeitpunktes (für alle Unterschiede $p>.05$ ). Nur für die Viertklässler des fünften Messzeitpunkts konnten mit $t(19)=2.82(p=.01)$ bedeutsame Differenzen in den Reproduktionsleistungen der ersten und dritten Liste verzeichnet werden. Analysen ergaben jedoch an mehreren Stellen signifikante Unterschiede der durchschnittlichen Rehearsal-SetGröße zwischen den drei Listen eines Messzeitpunktes. Für die Longitudinalgruppe zeigte sich dies beim dritten und vierten Messzeitpunkt jeweils zwischen Liste 2 und 3 sowie beim vierten Messzeitpunkt zwischen der ersten und dritten Liste. Bei den QuerschnittKontrollgruppen gab es bei den Viertklässlern des ersten und den Sechstklässlern des fünften Erhebungszeitpunktes Unterschiede in den durchschnittlichen Set-Größen der zweiten und dritten Liste und darüber hinaus für die erste Gruppe auch zwischen erster und zweiter Liste (für alle Unterschiede $p s \leq .012$; paarweise $t$-Tests). Es konnten jedoch keine Belege für eine systematische Variation der beiden Abhängigen Variablen über die drei Listen eines Messzeitpunktes und in keinem Fall korrespondierende Rehearsal-Set-Recall-Differenzen bedeutsamen Ausmaßes verzeichnet werden. Die berichteten Differenzen scheinen eher auf motivationale Faktoren oder Ermüdungseffekte zurückzugehen. Da inferenzstatistische Analysen keinen Einfluss des Geschlechts auf die zentralen Abhängigen Variablen finden konnten, wurde in allen weiteren Berechnungen auf eine Geschlechtsdifferenzierung verzichtet.

Die Auswertung erfolgte über Korrelationsanalysen sowie Varianzanalysen mit anschließenden Mittelwertsvergleichen ( $t$-Tests) oder Post-hoc Mehrfachvergleichen (SchefféTest). Zur Identifikation von Verlaufstypen wurde weiterhin eine explorative Konfigurationsfrequenzanalyse und zur Überprüfung von Häufigkeitsverteilungen ein nichtparametrischer Test eingesetzt (McNemar-Test). Wenn nicht anders berichtet, wurde für alle durchgeführten Tests das Signifikanzniveau auf $\alpha=.05$ gesetzt. Multiple (paarweise) Mittelwertsvergleiche wurden wegen der Gefahr der Kumulation des Fehlers erster Art mit Hilfe der AlphaAdjustierung nach Bonferroni durchgeführt. Der $t$-Test wurde auch bei kleinen Stichprobenumfängen als Verfahren zur Überprüfung von Unterschiedshypothesen eingesetzt. Dies geschah hauptsächlich aus Gründen der Vergleichbarkeit bzw. Einheitlichkeit. Darüber hinaus 
reagiert der $t$-Test nach Bortz (1993) relativ robust auf eine mögliche Voraussetzungsverletzung durch kleine Stichprobengrößen.

\subsection{Deskriptive Ergebnisse und Querschnittanalyse}

\subsubsection{Psychologische Hypothese und Vorhersagen}

Aus den querschnittlichen Befunden, die bislang über die Entwicklung von Rehearsal, den alterskorrelierten Anstieg der Reproduktionsleistung und den Zusammenhang zwischen diesen beiden Faktoren vorliegen, kann für den Klassenvergleich der vorliegenden Studie folgende psychologische Hypothese formuliert werden (s.a. Abschn. 3): Es gibt eine alterskorrelierte Zunahme in der Qualität des Memorierverhaltens und der Gedächtnisleistung sowie einen mit dem Alter steigenden Zusammenhang zwischen diesen beiden Faktoren $\left(\mathrm{PH}_{1}\right)$. Aus dieser psychologischen Hypothese können auf der darunterliegenden Ebene folgende psychologischen Vorhersagen (PVs) abgeleitet werden:

- Ältere Kinder haben eine größere durchschnittliche Rehearsal-Set-Größe als jüngere Kinder $\left(\mathrm{PV}_{1,1}\right)$.

- Ältere Kinder haben eine bessere durchschnittliche Reproduktionsleistung als jüngere Kinder $\left(\mathrm{PV}_{1,2}\right)$.

- Der Zusammenhang zwischen diesen beiden Faktoren ist bei älteren Kindern höher als bei jüngeren Kindern $\left(\mathrm{PV}_{1,3}\right)$.

\subsubsection{Befunde}

Für beide Abhängigen Variablen zeigt sich ein alterskorrelierter Anstieg. Dies gilt sowohl für die längsschnittliche Betrachtung auf Gruppenebene (Tabelle 5.1) als auch für den querschnittlichen Gruppenvergleich (Tabelle 5.2). Der Vergleich der Längsschnittgruppe ( $\left.\mathrm{t}_{1}\right)$ mit der Zweitklässler-Kontrollgruppe zeigte trotz bedeutsamer Altersunterschiede (s.o. Abschn. 4.2) vergleichbare mittlere Reproduktionsleistungen $(t(98)=1.98, p=.05)$ und durchschnittliche Rehearsal-Set-Größen $(t(98)=1.59, p=.12)$. Dieser Befund lässt die Annahme zu, dass kein Kohorteneffekt vorliegt und dass es sich um eine repräsentative Ausgangsstichprobe handelt. 
Tabelle 5.1: Mittelwerte (und Standardabweichungen) der Reproduktionsleistung und der Rehearsal-Set-Größe für die Longitudinalgruppe $(n=80)$, getrennt nach Messzeitpunkten.

\begin{tabular}{|c|c|c|c|c|}
\hline Messzeitpunkt (t) & $\mathrm{t}_{1}$ & $\mathrm{t}_{2}$ & $\mathrm{t}_{3}$ & $\mathrm{t}_{4}$ \\
\hline Klassenstufe & 2. & 3. & 3. & 4. \\
\hline Reproduktion & $5.21(1.61)$ & $5.55(1.61)$ & $5.85(1.49)$ & $6.31(1.39)$ \\
\hline Rehearsal-Set & $1.11(.86)$ & $1.40(.94)$ & $1.70(1.20)$ & $2.00(1.40)$ \\
\hline
\end{tabular}

Tabelle 5.2: Mittelwerte (und Standardabweichungen) der Reproduktionsleistung und der Rehearsal-Set-Größe für die Querschnittgruppen.

\begin{tabular}{|c|c|c|c|}
\hline Klassenstufe & $\begin{array}{c}2 . \\
(\mathrm{n}=20)\end{array}$ & $\begin{array}{c}4 . \\
(\mathrm{n}=40)\end{array}$ & $\begin{array}{c}6 . \\
(\mathrm{n}=40)\end{array}$ \\
\hline Reproduktion & $4.43(1.41)$ & $6.31(1.55)$ & $7.70(1.42)$ \\
\hline Rehearsal-Set & $1.45(.75)$ & $1.47(1.06)$ & $2.67(1.37)$ \\
\hline
\end{tabular}

Die inferenzstatistische Prüfung der Gruppenunterschiede zwischen den Kindern der Longitudinalgruppe am Ende der zweiten Klasse $\left(\mathrm{t}_{1}\right)$ und Viert- und Sechstklässlern der Querschnittgruppen bestätigte die Differenzen in der Lernleistung $(F(2,157)=34.82, p<\alpha)$ und der mittleren Set-Größe $(F(2,157)=29.44, p<\alpha)$. Erwartungsgemäß fielen diese Effekte etwas stärker aus als in der Studie von Guttentag et al. (1987), da diese mit Drittklässlern als jüngster Altersgruppe durchgeführt wurde. Drittklässler befinden sich bereits auf einem höheren strategischen Niveau als die Zweitklässler in der vorliegenden Studie. Die Kinder der Longitudinalgruppe wiesen beim vierten Messzeitpunkt bereits äquivalente Reproduktionsleistungen und sogar signifikant größere durchschnittliche Rehearsal-Sets $(t(118)=-2.24, p<\alpha)$ als die ein gutes halbes Jahr älteren Kinder der Querschnitt-Kontrollgruppe auf. Dies kann als Hinweis darauf interpretiert werden, dass durch die wiederholte Testung beim Längsschnitt Übungs- bzw. Vertrautheitseffekte auftreten.

Um eine über die formulierten Hypothesen hinausgehende Betrachtung der Leistungsunterschiede zwischen den Klassen zu ermöglichen, wurden Analysen auf der Ebene der 
Postionskurven angeschlossen. Der in Abbildung 5.1 dargestellte Verlauf der Reproduktionskurven und die unterschiedlichen Reproduktionswahrscheinlichkeiten für die drei Gruppen machen deutlich, dass der Leistungsvorteil älterer Kinder auf fast allen Listenpositionen mit Ausnahme von Position 6 vorhanden ist $(t \mathrm{~s} \geq 2.49, p \mathrm{~s}<\alpha)$, wobei die Unterschiede zwischen Viert- und Sechstklässlern auf die ersten drei Positionen beschränkt bleiben $(t \mathrm{~s}(78) \geq 3.38$, $p_{s}<\alpha$ ). Dieser Befund deckt sich mit der Annahme Ornsteins (z.B. Ornstein, Naus \& Liberty, 1975; Ornstein, Naus \& Stone, 1977), dass sich aktivere Memoriertechniken in Form von kumulativem Rehearsal vor allem auf den Abruf früher und mittlerer Listenitems auswirken (prerecency sections) und dass beim Vergleich verschiedener Klassenstufen auf Gruppenebene kaum Unterschiede in der Abrufwahrscheinlichkeit der letzten Listenitems gefunden werden (z.B. Ornstein \& Naus, 1985; Ornstein et al., 1985). Der einzig widersprüchliche Aspekt in den aktuellen Befunden war die mit $t(118)=1.88(p=.06)$ geringe Differenz zwischen Zweit- und Viertklässlern bei Position 1.

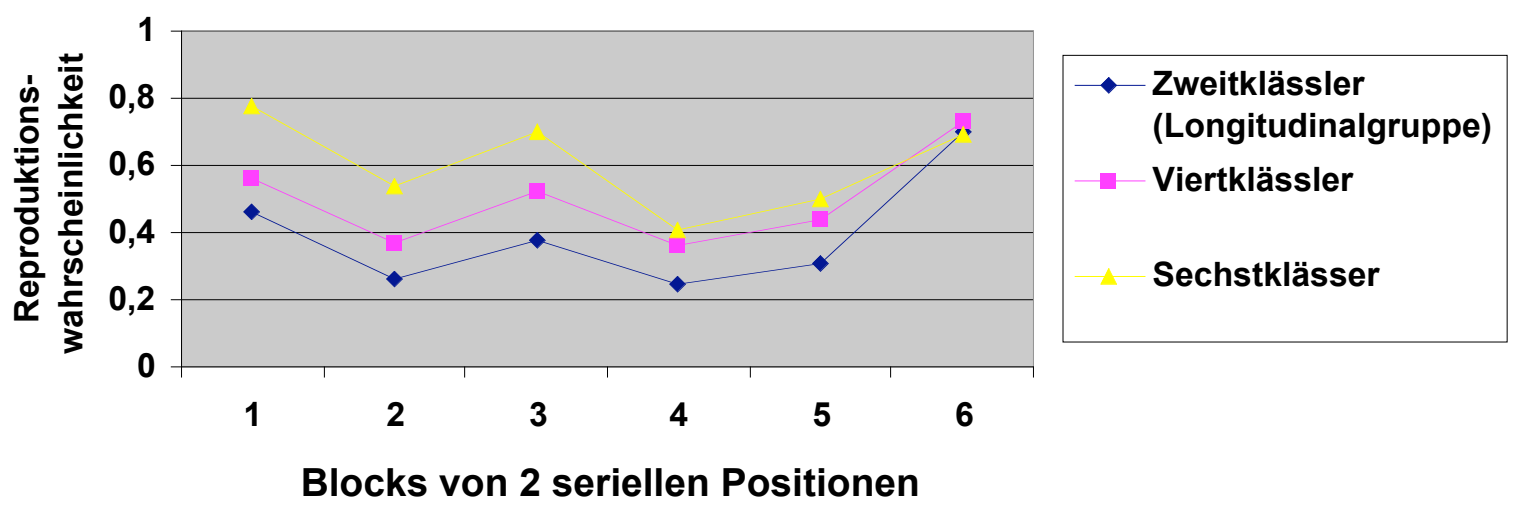

Abbildung 5.1: Reproduktionswahrscheinlichkeit serieller Itempositionen - Klassenvergleich.

Bei Messzeitpunkt 1 wurden 14 Items pro Lernliste präsentiert, ab Messzeitpunkt 2 waren es 12 Items je Trial. Für die Darstellung in Abbildung 5.1 und alle weiteren seriellen Positionskurven wurden zur besseren Vergleichbarkeit der Erhebungszeitpunkte für Messzeitpunkt 1 die Positionen 4 und 5, die sich in der Reproduktionswahrscheinlichkeit nicht unterschieden, gemittelt.

Die Analyse des Zusammenhangs zwischen Reproduktionsleistung und durchschnittlicher Set-Größe zeigte ein von früheren Arbeiten abweichendes Befundmuster. Spearman's rho wies für die Zweitklässler einen Wert von $r=.02(p=.89)$, also praktisch eine Nullkorrelation auf. Auch für die Gruppe der Viertklässler war der Zusammenhang der beiden Variablen mit $r=.10(p=.54)$ nicht statistisch bedeutsam. Bei den Sechsklässler konnte sogar ein 
leicht negativer Zusammenhang verzeichnet werden $(r=-.086, p=.60)$, der aber streng genommen auch einer Nullkorrelation entspricht. Weiterhin existierten keine bedeutsamen Unterschiede zwischen den Korrelationskoeffizienten ( $t \mathrm{~s} \leq .53 /-.80, p \mathrm{~s} \geq .42)$.

Der klassisch-konventionelle Vergleich der drei Altersgruppen erbrachte die vertrauten Differenzen in der durchschnittlichen Reproduktionsleistung und der Rehearsal-Set-Größe zwischen den drei Klassen. Auf dieser Betrachtungsebene konnte für keine Altersstufe ein bedeutsamer Zusammenhang zwischen den beiden Variablen und damit kein Einfluss des Memorierverhaltens auf die Reproduktionsleistung nachgewiesen werden. In der abschließenden Diskussion werden hierzu Überlegungen angestellt, um das Befundmuster zu erklären.

\subsection{Longitudinalanalyse auf Gruppenebene}

\subsubsection{Psychologische Hypothesen und Vorhersagen}

Im Gegensatz zu den bisherigen Betrachtungen erlauben längsschnittliche Analysen, die altersabhängigen Veränderungen der allgemeinen Gedächtnisvariablen, Reproduktionsleistung und durchschnittliche Set-Größe sowie ihren Zusammenhang zu überprüfen. Erneut kommt damit die bereits oben formulierte Annahme über die alterskorrelierte Zunahme der Qualität des Memorierverhaltens und der Gedächtnisleistung sowie ein mit dem Alter steigender Zusammenhang zwischen diesen beiden Faktoren $\left(\mathrm{PH}_{1}\right)$ zum Tragen. Darüber hinaus können ausgehend von den Befunden Kunzingers (1985) hohe zeitliche Stabilitäten im Memorierverhalten und der Gedächtnisleistung erwartet werden $\left(\mathrm{PH}_{2}\right)$. Aus diesen Psychologischen Hypothese können fünf Psychologischen Vorhersagen (PVs) abgeleitet werden:

- Mit zunehmendem Alter weisen Kinder größere durchschnittliche Rehearsal-Sets auf $\left(\mathrm{PV}_{1,1}\right)$.

- Mit zunehmendem Alter haben Kinder eine bessere durchschnittliche Reproduktionsleistung $\left(\mathrm{PV}_{1,2}\right)$.

- Der Zusammenhang zwischen diesen beiden Faktoren ist mit zunehmendem Alter größer $\left(\mathrm{PV}_{1,3}\right)$.

- Es bestehen zeitliche Stabilitäten zwischen den Set-Größen verschiedener Messzeitpunkte $\left(\mathrm{PV}_{2,1}\right)$.

- Es bestehen zeitliche Stabilitäten zwischen den Reproduktionsleistungen verschiedener Messzeitpunkte $\left(\mathrm{PV}_{2,2}\right)$. 


\subsubsection{Befunde}

Es fanden sich für die längsschnittliche Betrachtung auf Gruppenebene kontinuierliche Zuwächse in der Lernleistung und der Rehearsal-Set-Größe (Abbildung 5.2, s.a. Tabelle 5.1). Die Analyse der Entwicklung vom Ende der zweiten bis zum Anfang der vierten Klasse zeigte für die 80 Kinder der Längsschnittgruppe einen deutlichen Anstieg der Reproduktionsleistung $(F(3,237)=18.51, p<\alpha)$ und der durchschnittlichen Set-Größe $(F(3,237)=27.26, p<\alpha)$. Anschließende Mittelwertsvergleiche ergaben bedeutsame Leistungsdifferenzen $(t \mathrm{~s}(79) \geq 2.20$, $\left.p_{\mathbf{S}}<\alpha\right)$ und Steigerungen der durchschnittlichen Set-Größe $(t \mathrm{~s}(79) \geq 2.98, p \mathbf{s}<\alpha)$ zwischen jeweils benachbarten Messzeitpunkten. Ähnlich wie in der Studie von Kunzinger (1985) verringerte sich die Variabilität in der durchschnittlichen Reproduktionsleistung im Laufe der Zeit. Ebenfalls übereinstimmend mit der Literaturlage war der zeitgleiche Anstieg der Standardabweichung in der Rehearsal-Set-Größe (vgl. Tabelle 5.1). Dies spiegelte die zunehmende Variabilität in der Strategieproduktion im Verlauf der späteren Grundschuljahre wider.

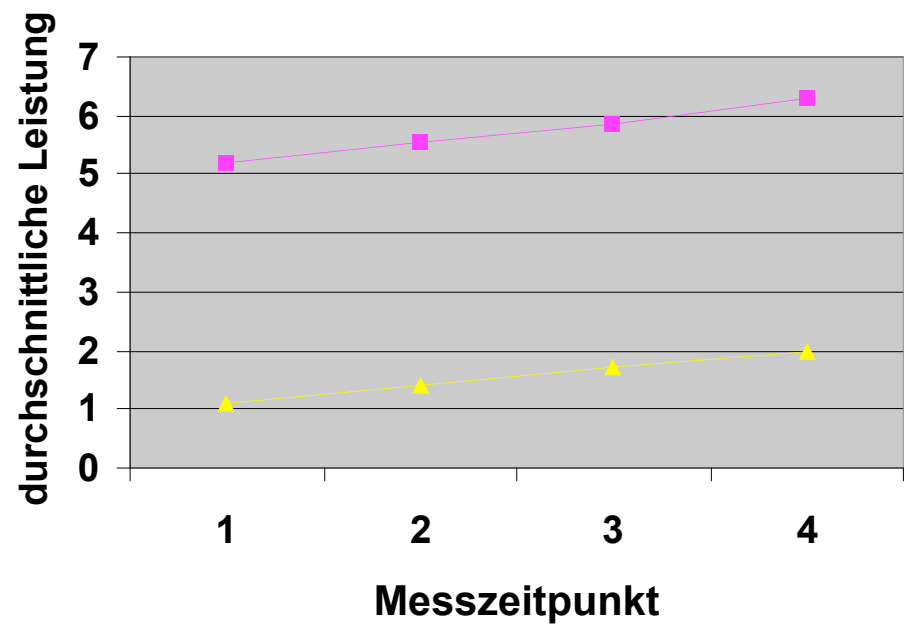

\begin{tabular}{|l|}
- Reproduktions \\
leistung \\
Reharsal-Set- \\
Größe
\end{tabular}

Abbildung 5.2: Mittlere Reproduktionsleistung und Set-Größe über die Messzeitpunkte.

Die Betrachtung der in Tabelle 5.3 dargestellten zeitlichen Stabilitäten der RecallLeistung und der durchschnittlichen Rehearsal-Set-Größe zeigte für beide Variablen signifikante Interkorrelationen zwischen den einzelnen Messzeitpunkten, die auf eine gewisse zeitliche Stabilität dieser Charakteristika zwischen 8 und 10 Jahren hindeuten.

Sie fiel dabei für die Set-Größe etwas höher aus, lag jedoch weit unter der von Kunzinger (1985) berichteten Zwei-Jahres-Stabilität für rehearsal set size zwischen sieben und neun Jahren von $r=.80$. Die Ein-Jahres-Stabilität war für beide Variablen zu Beginn des erfassten Zeitraums höher und sank speziell bei der Recall-Leistung im späteren Zeitraum deutlich ab. Die Korrelationskoeffizienten für die Reproduktionsleistung von aneinandergrenzenden 
Messzeitpunkten befanden sich bei durchschnittlich $r=.59$, was der von Kunzinger (1985) berichten Stabilität von $r=.60$ in etwa entsprach. Die Gesamtstabilität über die betrachteten eineinhalb Jahre war mit $r=.46$ jedoch deutlich niedriger.

Tabelle 5.3: Zeitliche Stabilität der Reproduktionsleistung und der Rehearsal-Set-Größe - Longitudinalgruppe.

\begin{tabular}{|l|c|c|c|}
\hline & Recall $_{1}$ & Recall $_{2}$ & Recall $_{3}$ \\
\hline Recall $_{2}$ & $.56^{* *}$ & & \\
\hline Recall $_{3}$ & $.59^{* *}$ & $.67^{* *}$ & \\
\hline Recall $_{4}$ & $.46^{* *}$ & $.50^{* *}$ & $.55^{* *}$ \\
\hline
\end{tabular}

\begin{tabular}{|c|c|c|c|}
\hline & Rehearsal-Set $_{1}$ & Rehearsal-Set $_{2}$ & Rehearsal-Set $_{3}$ \\
\hline Rehearsal-Set $_{2}$ & $.67^{* *}$ & & \\
\hline Rehearsal-Set $_{3}$ & $.58^{* *}$ & $.65^{* *}$ & \\
\hline Rehearsal-Set $_{4}$ & $.52^{* *}$ & $.57^{* *}$ & $.73^{* *}$ \\
\hline
\end{tabular}

$* *: p<\alpha=.01$

Speziell für die Strategievariable fanden sich relativ hohe Stabilitäten über alle Messzeitpunkte, was signalisiert, dass die Kinder, die früh größere Rehearsal-Sets und damit ein elaborierteres Lernverhalten zeigten, ihre Position innerhalb der Gruppe verhältnismäßig gut gewahrt hatten. Im Gegensatz zu den Befunden für die Strategie des kategorialen Organisierens scheint sich der Erwerb komplexer Memoriertechniken also nicht in Form von Sprüngen wie z.B. Alles-oder-Nichts bzw. durch einen Achterbahn-Verlauf zu vollziehen (vgl. Schneider \& Sodian, 1997; Sodian \& Schneider, 1999).

Tabelle 5.4: Zusammenhang zwischen Reproduktionsleistung und Rehearsal-Set-Größe - Longitudinalgruppe.

\begin{tabular}{|c|c|c|c|c|}
\hline & Set-Größe $_{1}$ & Set-Größe & Set-Größe $_{3}$ & Set-Größe $_{4}$ \\
\hline Recall $_{1}$ & .02 & & & \\
\hline Recall $_{2}$ & & $.22^{*}$ & & \\
\hline Recall $_{3}$ & & & .14 & \\
\hline Recall $_{4}$ & & & & $.28^{*}$ \\
\hline
\end{tabular}

$*: p<\alpha=.05$

Nach den Befunden der Arbeitsgruppe um Ornstein (z.B. Ornstein, Naus \& Stone, 1977) soll sich die zunehmende strategische Kompetenz älterer Kinder in einem größeren Ausmaß des Zusammenhangs zwischen der Set-Größe und der Reproduktionsleistung zeigen. Während beim ersten und dritten Messzeitpunkt praktisch Nullkorrelationen auftraten, erwiesen sich die Korralationskoeffizienten bei Messzeitpunkt 2 mit $r=.22$ und bei Messzeitpunkt 4 mit $r=.28$ als statistisch bedeutsam (Tabelle 5.4). Dass der Rückgang der Korrelation zum 
dritten Messzeitpunkt zeitlich mit einer vergleichsweise geringeren Stabilität der Strategievariablen (s. Tabelle 5.3) zusammenfiel, signalisiert, dass es im Verlauf der dritten Klasse zur verstärkten Entdeckung bzw. Nutzung von kumulativen Wiederholprozessen kam, ohne dass damit ein entsprechender Leistungsgewinn verbunden war. Dieser stellte sich unter Umständen erst bei Messzeitpunkt 4 ein, was sich dann in einer verhältnismäßig höheren Korrelation zwischen den beiden Maßen niederschlug.

Analog zum querschnittlichen Gruppenvergleich sollte sich die Zunahme an kumulativem Rehearsal vor allem in einer erhöhten Reproduktionswahrscheinlichkeit früher und mittlerer Listenitems zeigen. Entsprechend der zuvor formulierten Annahme wäre hier eine deutliche Zunahme der Reproduktionswahrscheinlichkeit von Anfangsitems zwischen Messzeitpunkt 2 und 3 als Folge einer verbesserten strategischen Performanz zu erwarten. Die Betrachtung der in Abbildung 5.3 dargestellten Reproduktionskurven und Reproduktionswahrscheinlichkeiten zu den vier Messzeitpunkten erbrachte für alle Messzeitpunkte ähnliche Verlaufskurven mit signifikant unterschiedlichen Recall-Wahrscheinlichkeiten zwischen benachbarten Listenpositionen ( $t \mathrm{~s} \geq 2.24, p s \leq \alpha)$. Die einzige Ausnahme bildete die Differenz zwischen Position 4 und 5, die nur beim ersten Messzeitpunkt statistisch bedeutsam war.
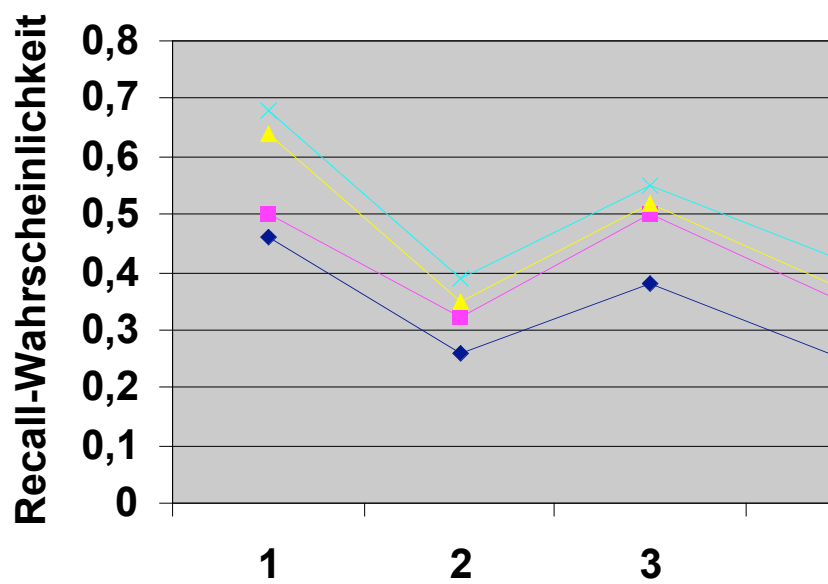

Blocks von 2 seriellen Positionen

Abbildung 5.3: Positionskurven für die vier Messzeitpunkte (t) - Longitudinalgruppe.

Analysen der Abweichungen in den Reproduktionswahrscheinlichkeiten einer Listenposition zwischen Messzeitpunkten differenzierten dieses Bild weiter. Bei Position 1 trat der größte Sprung zwischen Messzeitpunkt 2 und 3 auf $(t(79)=4.5, p<\alpha)$, was wiederum für eine Entwicklung der strategischen Performanz im Verlauf des dritten Schuljahres spricht. Bei der zweiten bis fünften Listenposition zeigten sich nur zwischen dem ersten und zweiten Erhe- 
bungszeitpunkt signifikante Differenzen auf Gruppenebene $(t s \geq 2.16, p s<\alpha)$. Darüber hinaus ließen sich bei keiner Listenposition bedeutsame Zuwächse zwischen aufeinander folgenden Messzeitpunkten finden. Besonders die Reproduktionswahrscheinlichkeit der letzten beiden Listenitems, die durch Position 6 repräsentiert wurden, blieben über die Zeit weitgehend unverändert ( $t \mathrm{~s} \leq .93, p s \geq .36)$.

Auch auf der längschnittlichen Betrachtungsebene konnten ein genereller und kontinuierlicher Anstieg der Gedächtnisleistung und der Rehearsal-Set-Größe verzeichnet werden. Beide Komponenten zeigten eine bedeutsame Halbjahres- und Gesamtzeit-Stabilität. Allerdings fiel das Ausmaß an Labilität (vgl. Kunzinger, 1985) über die Gesamtzeit, nachgewiesen durch Spearman's rho von $r=.46$ für die Lernleistung und $r=.52$ für die strategische Komponente, deutlich höher aus als in der Studie von Kunzinger (1985). Aufgrund der größeren Stichprobe waren Korrelationswerte wie von Kunzinger (1985) berichtet allerdings auch nicht zu erwarten. Diese ,across-age variability shown in an individual's relative standing within the referent group“ (Kunzinger, 1985, S. 644) gilt nach Wohlwill (1973) als allgemeiner Indikator der individuellen Stabilität bzw. Labilität eines Merkmals.

Der Zusammenhang zwischen der Gedächtnis- und der Strategievariablen stieg über die Gesamtzeit an. Der Einbruch bei Messzeitpunkt 3 legt die Annahme von qualitativen Veränderungen des Rehearsal-Verhaltens im Verlauf der dritten Klasse nahe. Gestützt wird diese Vermutung durch korrespondierende Zuwächse in der Reproduktionswahrscheinlichkeit von Anfangsitems beim dritten Messzeitpunkt.

\subsection{Analyse der intraindividuellen Entwicklungsverläufe}

\subsubsection{Psychologische Hypothesen und Vorhersagen}

Auf der nächsten Betrachtungsebene werden individuelle Entwicklungsanalysen berichtet, die eine differentielle Betrachtung der interindividuellen Unterschiede und intraindividuellen Variabilität der Entwicklungsphänome erlauben. Um die individuelle strategische Performanz und deren Veränderung näher charakterisieren zu können, wurden neben der Berechnung der Häufigkeit, mit der eine Strategie im Verlauf eines Erhebungszeitpunkts aufgetreten war, zwei Kategorisierungswege strategischer Performanz gewählt: In einem ersten Schritt wurden die Kinder entsprechend ihrer primär genutzten Lernstrategie klassifiziert. In einem zweiten Schritt erfolgte die Klassifikation der Kinder nach dem individuellen Rehearsal-Typ bzw. -Muster. Dafür wurde die von Guttentag et al. (1987) vorgeschlagene Kategori- 
sierung variiert und die Kinder entsprechend ihrer durchschnittlichen Rehearsal-Set-Größe einer von drei Gruppen zugewiesen:

- $\quad$ Gruppe 1: „Non-cumulative“-Rehearser $=$ mittleres Rehearsal-Set $\leq 1,25$

- $\quad$ Gruppe 2: „Inconsistent“-Rehearser = mittleres Rehearsal-Set zwischen 1,25 und 2,25

- Gruppe 3: „Dominant-cumulative“-Rehearser $=$ mittleres Rehearsal-Set $\geq 2,25$

Auch auf der individuellen Ebene wird eine alterskorrelierte Zunahme in der Qualität des Memorierverhaltens und der Gedächtnisleistung sowie ein mit dem Alter steigender Zusammenhang zwischen diesen beiden Faktoren $\left(\mathrm{PH}_{\mathrm{l}}\right)$ angenommen. Darüber hinaus sollten sich hohe zeitliche Stabilitäten bezüglich des Memorierverhaltens und der Gedächtnisleistung zeigen $\left(\mathrm{PH}_{2}\right)$. Entprechend der vorgenommenen Operationalisierung können aus diesen Hypothesen folgende empirische Vorhersagen abgeleitet werden:

- In dem betrachteten Altersabschnitt kommt es zu einem bedeutsamen Anstieg an Kindern, die als „Dominant-cumulative“-Rehearser klassifiziert werden können. $\left(\mathrm{PV}_{1,1}\right)$.

- Parallel dazu kommt es zu einer besseren Reproduktionsleistung $\left(\mathrm{PV}_{1,2}\right)$.

- Zwischen diesen beiden Entwicklungen besteht ein Zusammenhang in der Form, dass „Dominant-cumulative“-Rehearser bedeutsam höhere Reproduktionsleistungen erbringen als Kinder, die nicht als „Dominant-cumulative“-Rehearser klassifiziert wurden $\left(\mathrm{PV}_{1,3}\right)$.

- Die zeitliche Stabilität (bivariate Korrelation) der Rehearsal-Klassifikation ist über die Gesamtzeit und zwischen einzelnen Erhebungszeitpunkten statistisch bedeutsam $\left(\mathrm{PV}_{2,1}\right)$.

- Die zeitliche Stabilität (bivariate Korrelation) der Behaltensleistung ist über die Gesamtzeit und zwischen einzelnen Erhebungszeitpunkten statistisch bedeutsam $\left(\mathrm{PV}_{2,2}\right)$.

Weiterhin werden vertiefende Analysen präsentiert, um der Frage nachzugehen, ob es zu Rehearsal-initiierten Abrufphänomenen kommt. Denkbar wären hier Memorierprozesse während des Intervalls, das der Abrufphase vorausgeht. Durch kumulatives Rehearsal in der Lernphase könnten auch strategische Prozesse in der Abrufphase hervorgerufen werden, die sich z.B. in Form von kürzeren Latenzzeiten oder Organisationsprozessen in der OutputPhase zeigen (vgl. Abschnitt 2.1.3).

\subsubsection{Befunde}

Deskriptive Befunde: Die Visualisierung der genutzten Strategien in Abbildung 5.4 zeigt, dass die einmalige Nennung (Labeling) als häufigste Strategie angewandt wurde, gefolgt von kumulativem Rehearsal. Die Strategie des singulären Rehearsals, die nach den Be- 
funden aus Querschnittstudien in dem Alterszeitraum zwischen 8 und 10 Jahren die dominierende Mnemotechnik darstellen soll, fand sich jedoch nur zu einem geringen Prozentsatz, ebenso wie Assoziationen. Elaborationen waren mit Häufigkeiten zwischen 1 und 4 Prozent kaum vertreten. Insgesamt verschoben sich die Häufigkeiten über den erfassten Zeitraum nur gering. Lediglich die Anzahl der Kinder, die die Strategie des kumulativen Rehearsals nutzten, stieg von Ende der zweiten bis Anfang der vierten Klasse.
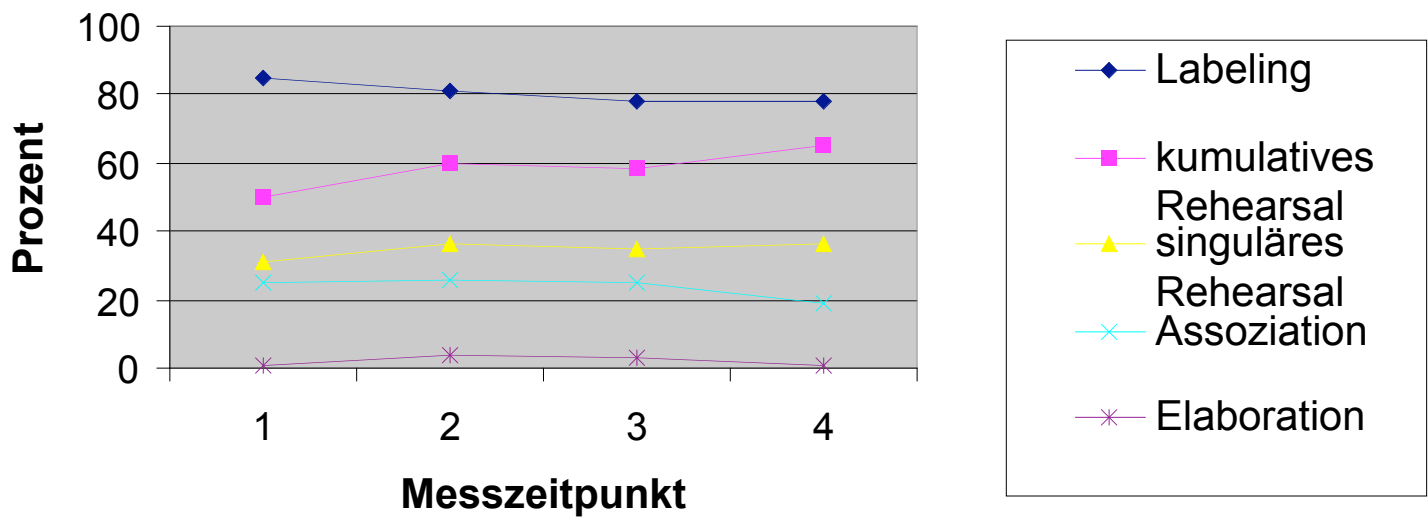

Abbildung 5.4: Prozentsatz von Kindern, die eine Strategie zu einem Messzeitpunkt angewandt haben (Mehrfachnennungen möglich).

Viele Kinder wendeten mehrere Gedächtnisstrategien an, selbst wenn eine Mnemotechnik als dominierendes Lernverhalten vorlag. Nähere Analysen zeigten, dass am Ende der 2. Klasse lediglich 28 Kinder (35\%) nur eine Strategie nutzten. Für die restlichen 52 Kinder $(65 \%)$ konnte ein multipler Strategieeinsatz mit $\geq 2$ Mnemotechniken beobachtet werden. Am Anfang und Ende der dritten Klasse waren es 57 Kinder (71\%) bzw. 52 Kinder (65\%), die mehr als eine Gedächtnisstrategie gebrauchten. $\mathrm{Zu}$ Beginn der vierten Klasse belief sich diese Zahl dann auf 56 Kinder (70\%).

Das Bild über das allgemeine Lernverhalten der Kinder in der Längsschnittgruppe wird ergänzt durch die Angabe der Häufigkeit, mit der Kinder eine Strategie zu einem der vier Messzeitpunkte als Hauptstrategie nutzten (s. Tabelle 5.5). Eine Hauptstrategie ist dabei als Lernverhalten definiert, das in mehr als 50 Prozent der Lernmöglichkeiten angewandt wurde. Wegen der geringen Bedeutung von Elaborationen für die Bearbeitung der Aufgabe wurden Assoziationen und Elaborationen hier in einer gemeinsamen Kategorie erfasst. 
Tabelle 5.5: Anzahl (und Prozentsatz) von Kindern in den Hauptstrategie-Gruppen, getrennt nach Messzeitpunkten ( $\mathrm{t}$ ).

\begin{tabular}{|c|c|c|c|c|c|}
\hline & $\begin{array}{c}\text { keine Haupt- } \\
\text { strategie }\end{array}$ & Labeling & $\begin{array}{c}\text { singuläres } \\
\text { Rehearsal }\end{array}$ & $\begin{array}{c}\text { kumulatives } \\
\text { Rehearsal }\end{array}$ & $\begin{array}{c}\text { Assoziation- } \\
\text { Elaboration }\end{array}$ \\
\hline $\mathrm{t}_{1}$ & $26(33 \%)$ & $33(41 \%)$ & $5(6 \%)$ & $11(14 \%)$ & $5(6 \%)$ \\
\hline $\mathrm{t}_{2}$ & $17(21 \%)$ & $24(30 \%)$ & $9(11 \%)$ & $20(25 \%)$ & $10(12 \%)$ \\
\hline $\mathrm{t}_{3}$ & $14(18 \%)$ & $21(26 \%)$ & $8(10 \%)$ & $27(34 \%)$ & $10(12 \%)$ \\
\hline $\mathrm{t}_{4}$ & $8(10 \%)$ & $20(25 \%)$ & $11(14 \%)$ & $34(43 \%)$ & $7(9 \%)$ \\
\hline
\end{tabular}

Der Anteil von Kindern, die eine der aufgeführten Strategien als primäre Lernmöglichkeit nutzten, stieg über die Messzeitpunkte. Vor allem der Anteil von Kindern, die kumulatives Rehearsal mit einem Anteil von mehr als 50 Prozent anwandten, nahm deutlich zu. Auffällig ist, dass der Anteil an Kindern mit Labeling-dominiertem Lernverhalten im Gesamtvenlauf hoch war, wohingegen die Anzahl an Kindern mit singulärem Rehearsal als Hauptstrategie gering ausfiel. Letzteres steht erneut im Gegensatz zu früheren Befunden (z.B. Guttentag et al., 1987; Ornstein \& Naus, 1978), nach denen in der zweiten und dritten Klasse primär single word rehearsal angewandt wird.

Für drei Messzeitpunkte konnten keine statistisch bedeutsamen Unterschiede in den Reproduktionsleistungen zwischen den Hauptstrategie-Gruppen gefunden werden $\left(F_{1}(4,75)=1.32, p=.27 ; F_{2}(4,75)=1.58, p=.50\right.$ und $\left.F_{4}(4,75)=1.30, p=.28\right)$. Nur beim dritten Erhebungszeitpunkt zeigte sich mit $F(4,75)=2.99(p<\alpha)$ ein signifikanter Unterschied. Ein anschließender Scheffé Post-hoc Vergleich wies auf eine statistisch bedeutsame Leistungsdifferenz zwischen der Gruppe der singulären Rehearser und der Gruppe der kumulativen Rehearser hin $(p<\alpha)$.

Insgesamt kann mit Spearman's rho $r \geq .274(p<.01)$ zwischen allen Messzeitpunkten von einer hohen zeitlichen Stabilität der Zuordnung zu den Hauptstrategiegruppen gesprochen werden. Die Stabilitäten zwischen aufeinander folgenden Messzeitpunkten (Halbjahresstabilitäten) lagen bei $r_{1-2}=.31, r_{2-3}=.27$ und $r_{3-4}=.43$ (alle $p_{\mathrm{s}}<.01$ ). Die im Verhältnis geringe und auch insgesamt niedrigste Stabilität zwischen Messzeitpunkt 2 und 3 legt die Annahme qualitativer Veränderungen in Form eines Wechsels des strategischen Verhaltens im Verlauf der dritten Klasse nahe, die zu einer Verschiebung in der Gruppenzuordnung führten.

Die Analyse qualitativer Entwicklungen: Da gerade diese qualitativen Veränderungen im Verlauf der späteren Grundschuljahre und deren intraindividuelle Veränderungen im Fokus der vorliegenden Arbeit stehen, wurden die Kinder in einem weiteren Schritt entsprechend ihrer durchschnittlichen Rehearsal-Set-Größe klassifiziert. Dafür wurden drei Gruppen gebildet, die sich hinsichtlich ihrer durchschnittlichen Rehearsal-Set-Größe unterschieden: a) 
„Non-cumulative“-Rehearser (mittleres Rehearsal-Set $\leq 1.25$ ), b) „Inconsistent“-Rehearser (mittleres Rehearsal-Set zwischen 1.25 und 2.25), sowie c) „Dominant-cumulative“Rehearser (mittleres Rehearsal-Set $\geq 2.25$ ).

Tabelle 5.6: Anzahl und Prozentsatz von Probanden in den Rehearsal-Typ-Gruppen, getrennt nach Messzeitpunkten ( $\mathrm{t}$ ).

\begin{tabular}{|c|c|c|c|}
\hline & "Non-cumulative“ & "Inconsistent" & $\begin{array}{c}\text { "Dominant- } \\
\text { cumulative“ }\end{array}$ \\
\hline $\mathrm{t}_{1}$ & $65(81 \%)$ & $3(4 \%)$ & $12(15 \%)$ \\
\hline $\mathrm{t}_{2}$ & $48(60 \%)$ & $18(22 \%)$ & $14(18 \%)$ \\
\hline $\mathrm{t}_{3}$ & $42(53 \%)$ & $13(16 \%)$ & $25(31 \%)$ \\
\hline $\mathrm{t}_{4}$ & $34(42 \%)$ & $15(19 \%)$ & $31(39 \%)$ \\
\hline
\end{tabular}

Wie aus Tabelle $5.6 \mathrm{zu}$ ersehen ist, stieg die Anzahl von Kindern, die als „Dominantcumulative"-Rehearser klassifiziert wurden mit zunehmendem Alter. Dies geschah am deutlichsten zwischen dem zweiten und dritten Messzeitpunkt. Analog dazu sank die Anzahl an „Non-cumulative“-Reheasern kontinuierlich; die Menge der „Inconsistent“-Rehearser blieb dagegen ab dem zweiten Messzeitpunkt weitgehend stabil. Mit 12 Kindern (15\%), die die Strategie des kumulativen Rehearsals als dominante Strategie bereits beim ersten Messzeitpunkt anwendeten, entsprach der aktuelle Befund dem in der Literatur berichteten Anteil an kumulativen Rehearsern in der zweiten Klassenstufe (z.B. Guttentag et al., 1987). Allerdings lag der Prozentsatz von kumulativen Rehearsal-Strategen bei den Viertklässlern deutlich unter den 71 Prozent, die in der Untersuchung von Guttentag et al. (1987) als Cumulative klassifiziert wurden.

Tabelle 5.7: Zeitliche Stabilität der Rehearsal-Typ-Klassifikation.

\begin{tabular}{|c|c|c|c|}
\hline & $\mathrm{t}_{1}$ & $\mathrm{t}_{2}$ & $\mathrm{t}_{3}$ \\
\hline $\mathrm{t}_{2}$ & $.54^{* *}$ & & \\
\hline $\mathrm{t}_{3}$ & $.57^{* *}$ & $.55^{* *}$ & \\
\hline $\mathrm{t}_{4}$ & $.48^{* *}$ & $.44^{* *}$ & $.69 * *$ \\
\hline${ }^{* *}: p<\alpha=.01 ; \mathrm{t}=$ Messzeitpunkt
\end{tabular}

Die Überprüfung der Zeitstabilität der Zuordnung zu Rehearsal-Typen (Tabelle 5.7) zeigte mit $r \mathrm{~s} \geq .54$ zwischen allen aufeinander folgenden Erhebungen Korrelationskoeffizienten bedeutsamen Ausmaßes. Die Fluktuation war jedoch bis zum dritten Messzeitpunkt noch vergleichsweise hoch. Danach scheint sich die Einteilung nach Rehearsal-Typen weitgehend gefestigt zu haben. 
In Tabelle 5.8 ist die Anzahl von Kindern aufgeführt, die zwischen aufeinander folgenden Messzeitpunkten ihre Position halten konnten bzw. die in der Klassifikation auf- oder abgestiegen sind. Es zeigte sich, dass vor allem zwischen den ersten beiden Messzeitpunkten eine größere Anzahl von Kindern (n=14) vom „Non-cumulative“- zu einem „Inconsistent“Status wechselte, wohingegen der größte Wechsel von den beiden anderen Gruppen zur Kategorie der „Dominant-cumulative“ Rehearser beim dritten $(n=13)$ und vierten $(n=10)$ Messzeitpunkt zu verzeichnen war. Dagegen fiel die Anzahl von Rückfällen auf ein geringeres strategisches Niveau mit $4\left(t_{1}=>t_{2}\right), 10\left(t_{2}=>t_{3}\right)$ und $7\left(t_{3}=>t_{4}\right)$ Kindern verhältnismäßig gering aus. Dies signalisiert einen generellen Trend zu einem elaborierteren strategischen Verhalten im Verlauf der späten Grundschuljahre. Der an anderer Stelle für die Strategie des kategorialen Organisierens berichtete abrupte Prozess von Strategieaneignung und -verlust (vgl. Schneider \& Sodian, 1997; Sodian \& Schneider, 1999) konnte in den vorliegenden Daten damit nicht gefunden werden.

Tabelle 5.8: Anzahl und Verlauf der Rehearsal-Typ-Zuweisung zwischen aufeinander folgenden Messzeitpunkten.

\begin{tabular}{|c|c|c|c|}
\hline & „Non-cumulative“ $t_{2}$ & „Inconsistent“ $t_{2}$ & „,Dom.-Cumulative“ $t_{2}$ \\
\hline „Non-cumulative“ $t_{1}$ & 46 & 14 & 5 \\
\hline „Inconsistent“ $t_{1}$ & 1 & 2 & 0 \\
\hline „Dom.-cumulative“ $t_{1}$ & 1 & 2 & 9 \\
\hline
\end{tabular}

\begin{tabular}{|c|c|c|c|}
\hline & „Non-cumulative“ $\mathrm{t}_{3}$ & „Inconsistent" $\mathrm{t}_{3}$ & „Dom.-Cumulative“ $\mathrm{t}_{3}$ \\
\hline „Non-cumulative“ $\mathrm{t}_{2}$ & 34 & 7 & 7 \\
\hline „Inconsistent“ $\mathrm{t}_{2}$ & 8 & 4 & 6 \\
\hline „Dom.-cumulative“ $\mathrm{t}_{2}$ & 0 & 2 & 12 \\
\hline
\end{tabular}

\begin{tabular}{|c|c|c|c|}
\hline & „Non-cumulative“ $t_{4}$ & „Inconsistent“ $t_{4}$ & „Dom.-Cumulative“ $t_{4}$ \\
\hline „Non-cumulative“ $t_{3}$ & 30 & 7 & 5 \\
\hline „Inconsistent“ $t_{3}$ & 3 & 5 & 5 \\
\hline „Dom.-cumulative“ $t_{3}$ & 1 & 3 & 21 \\
\hline
\end{tabular}

Die genauere Analyse der Entwicklungsmuster in den Verlaufsdaten erfolgte durch eine explorative Konfigurationsfrequenzanalyse (Krauth \& Lienert, 1973), um überzufällige Entwicklungsmuster, so genannte Entwicklungstypen, zu identifizieren. Neben den konsistenten „Non-cumulative“ (31\% der Probanden) und „Dominant-cumulative“-Rehearsern (11\%) (beide mit $p<.001)$ konnte ein Entwicklungstyp mit Kindern entdeckt werden, die beim ersten 
Messzeitpunkt zur Gruppe der „Non-cumulative“-Rehearser gehörten und ab Messzeitpunkt 2 zu den „Inconsistent“ gezählt wurden $(4 \%, p=.007)$. Alle anderen möglichen Kategorienmuster hatten eine Wahrscheinlichkeit von $p>\alpha$.

Weiterhin konnten mit dem Chi-Quadrat-Test nach McNemar zwei statistisch bedeutsame Entwicklungsschübe in den Verlaufsdaten identifiziert werden: der Wechsel von der „Non-cumulative“- zur „Inconsistent“-Gruppe beim Übergang zu Messzeitpunkt 2 und eine zweite Bewegung zum „Dominant-cumulative“-Typ beim Übergang zu Messzeitpunkt 3 $(p s<\alpha)$. Die Annahme eines generellen Rückgangs des strategischen Verhaltens auf einer bestimmten Entwicklungs- bzw. Klassenstufe konnte dagegen durch die Befunde nicht gestützt werden.

In verschiedenen Arbeiten war nachgewiesen worden, dass kumulative RehearsalProzesse zu besseren Reproduktionsleistungen führen (z.B. Guttentag et al., 1987; Ornstein \& Naus, 1978). Die Betrachtung der Reproduktionsleistungen der drei Rehearsal-Typ-Gruppen in Abbildung 5.5 zeigt einen Anstieg der Lernleistung in allen drei Gruppen über die Zeit, mit der Ausnahme, dass die Recall-Leistung in der Gruppe der „Inconsistent“-Rehearser zum dritten Messzeitpunkt leicht zurückging.

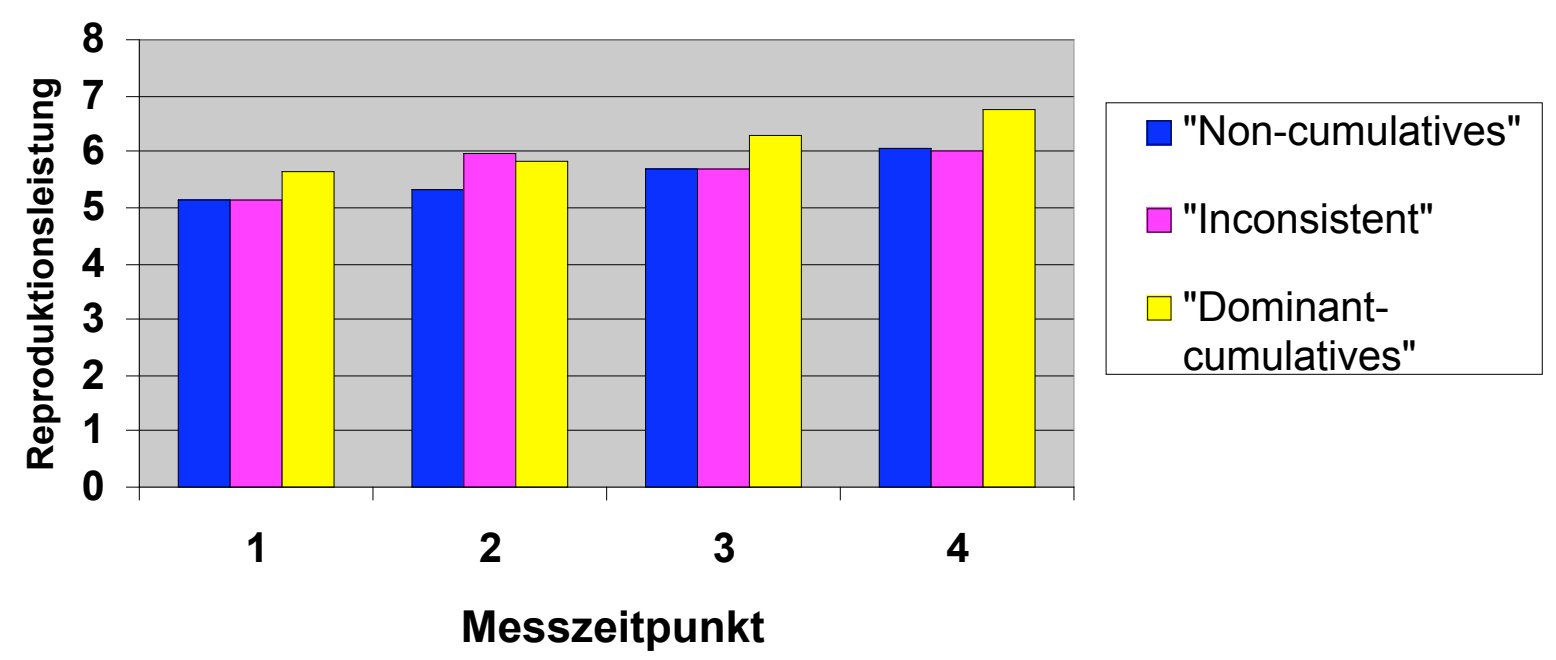

Abbildung 5.5: Reproduktionsleistung der drei Rehearsal-Typ-Gruppen, getrennt nach Messzeitpunkten.

Das erklärt sich dadurch, dass zu diesem Zeitpunkt ein größerer Anteil von vormals als „Inconsistent“ klassifizierten Kindern den Status von „Dominant-cumulative“-Rehearsern einnahm und damit ein qualitativ höheres Niveau strategischer Performanz erreichte. Ein signifikanter Leistungsvorteil der „Dominant-cumulative“-Gruppe gegenüber den „Noncumulative“-Rehearsern zeigte sich nur beim vierten Messzeitpunkt $(t(60)=2.07, p<\alpha)$. An- 
sonsten gab es keine statistisch bedeutsamen Unterschiede zwischen den Gruppen (alle $t \mathrm{~s} \leq 1.94, p \mathrm{~s} \geq .059)$.

Die Betrachtung der seriellen Positionskurven zeigte für alle Messzeitpunkte unterschiedliche Verlaufsformen zwischen den Rehearsal-Typ-Gruppen (vgl. Abbildung 5.6). Der deskriptiv ersichtliche Erinnerungsnachteil der „Dominant-cumulative“-Rehearser bei der letzten Listenposition widerspricht früheren Befunden der Arbeitsgruppe um Ornstein, wonach es im Recency-Bereich keine oder nur geringe Unterschiede zwischen den RehearsalTyp-Gruppen gibt. Um diesen überraschenden Befund statistisch abzusichern, wurden multivariate Varianzanalysen mit dem Rehearsal-Typ als Zwischensubjektfaktor gerechnet. Es fanden sich für alle Messzeitpunkte signifikante Haupteffekte des Rehearsal-Typs auf Listenposititon $6\left(F_{\mathrm{s}}(2,77) \geq 6.79, p_{\mathrm{s}}<\alpha\right)$. Darüber hinaus konnten durchgängig statistisch bedeutsame Werte im Primacy-Bereich $(F \mathrm{~s}(2,77) \geq 3.60, p \mathrm{~s}<\alpha)$ verzeichnet werden. Dies deckt sich mit der Annahme Ornsteins, dass sich der Leistungsvorteil durch die Anwendung von kumulativem Rehearsal vor allem im Bereich der frühen und mittleren Listenitems zeigt (vgl. auch Abbildung 5.6).

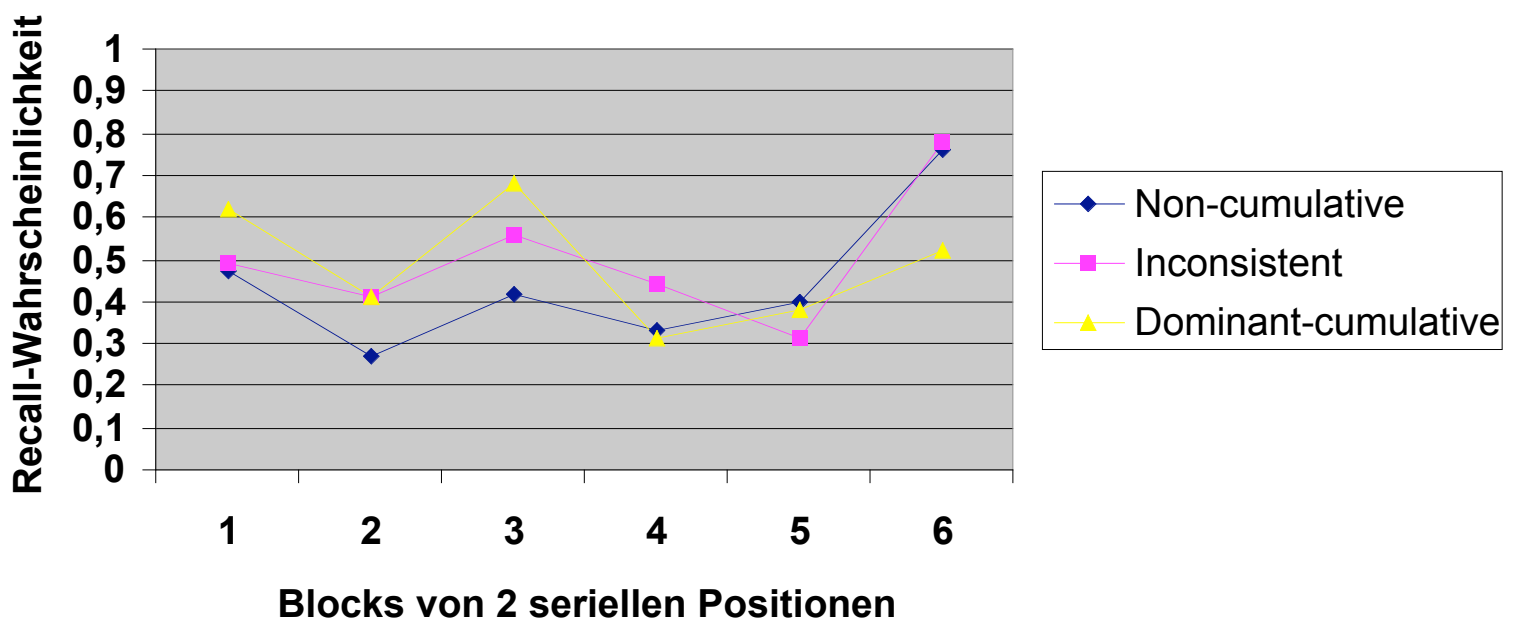

Abbildung 5.6: Exemplarische Darstellung der Reproduktionswahrscheinlichkeiten der Listenpositionen für die drei Rehearsal-Typ-Gruppen (2. Messzeitpunkt).

Anschließende paarweise Mittelwertsvergleiche ergaben durchgängig einen Leistungsvorteil der „Dominant-cumulative“- gegenüber den „Non-cumulative“-Rehearsern im Primacy-Bereich und eine umgekehrte Überlegenheit der „Non-cumulatives“ gegenüber den „Dominant-cumulatives“ am Ende der seriellen Positionskurve $(t \mathrm{~s} \geq 2.54, p \mathrm{~s}<\alpha)$. Beim zweiten Messzeitpunkt gab es Unterschiede zwischen den „Dominant-cumulative“- und den „Inconsistent"-Rehearsern zugunsten letzterer bei Listenposition $6(t(30)=3.15, p<\alpha)$ und zwischen 
der „Inconsistent"- und der „Non-cumulative“-Gruppe zugunsten ersterer bei Listenposition 3 $(t(64)=2.54, p<\alpha)$. Abbildung 5.6 veranschaulicht die gefundenen Verläufe exemplarisch für den zweiten Messzeitpunkt.

Dieses Ergebnismuster steht im Widerspruch zu den meisten bisherigen Befunden aus Querschnittsstudien, die keine bedeutsamen Unterschiede zwischen verschiedenen Gruppen von Strategen im Recency-Bereich gefunden haben. Ein mit dem vorliegenden primacyrecency trade-off vergleichbares Muster in der Studie von Ornstein, Naus und Stone (1977) wird von den Autoren durch Modalitätsaspekte der Präsentation erklärt. Danach kann eine akustische Darbietung von Lernitems, wie in der vorliegenden Studie, zu stärkeren Inferenzen zwischen aktuell memorierten und früheren Listenitems führen.

Die Betrachtung der seriellen Positionskurven offenbarte nicht nur Unterschiede der Reproduktionswahrscheinlichkeiten zwischen den Gruppen, sondern auch Differenzen zwischen den Reproduktionswahrscheinlichkeiten benachbarter Listenpositionen innerhalb der drei Gruppen (vgl. Abbildung 5.6). Besonders die hohe Reproduktionswahrscheinlichkeit bei Listenposition 3 widerspricht der Annahme, dass bei der freien Reproduktion einer seriellen Lernliste die Items aus dem Mittelbereich am wenigsten reproduziert werden.

Die 6 (serielle Position) x 3 (Rehearsal-Typ) varianzanalytischen Überprüfung dieser Verläufe mit der seriellen Position als Messwiederholungsfaktor zeigte statistisch bedeutsame Positionseffekte, die signalisierten, dass die Reproduktionswahrscheinlichkeiten der Listenpositionen unabhängig von der Einteilung in Typen signifikant variierten: $F_{1}(5,385)=14.05$, $F_{2}(5,385)=24.43, F_{3}(5,385)=40.02$ und $F_{4}(5,385)=40.65$ (alle $\left.p s<\alpha\right)$. Darüber hinaus gab es zu allen Messzeitpunkten eine bedeutsame Wechselwirkung zwischen der seriellen Position und der Rehearsal-Typ-Klassifikation: $F_{1}(10,385)=5.33, F_{2}(10,385)=4.54, F_{3}(10,385)=5.11$ und $F_{4}(10,385)=6.79($ alle $p s<\alpha)$. Die genauere Betrachtung der Mittelwertsunterschiede zwischen benachbarten Listenpositionen durch $t$-Tests zeigte nicht nur die erwarteten Positionseffekte (Primacy- und Recency-Effekte), sondern auch signifikante Differenzen zwischen benachbarten seriellen Positionen aus der Mitte der Liste. Besonders auffällig waren die hohen Reproduktionswahrscheinlichkeiten der dritten Listenposition.

Die Analyse von Abrufphänomenen: Die gefundenen mehrfach U-förmigen Verläufe in allen seriellen Positionskurven sind typisch für Itemlisten mit kategorier- bzw. gruppierbarem Material, da dort für jede Gruppe ein Primacy- und Recency-Effekt auftritt. Für Listen mit unverbundenem Material, wie es in der vorliegenden Untersuchung verwendet wurde, sind serielle Positionskurven mit einfach gebeugtem Muster zu erwarten (vgl. Ng \& Maybery, 2002). Das sowohl für jede einzelne Liste als auch für die gemittelten Werte gefundene, mul- 
tipel gezackte Muster lässt dagegen vermuten, dass durch die kumulative Memoriertätigkeit während der Lernphase Organisationsprozesse in der Abrufphase initiiert wurden, die zu Primacy- und Recency-Effekten innerhalb der Rehearsal-Sets führten.

Um zu prüfen, ob die unterschiedlichen Verläufe der Positionskurven durch das kumulative Rehearsal entstehen, wurden die Differenzen zwischen „Dominant-cumulative“- und „Non-cumulative“-Rehearsern zwischen der 2. und 3. sowie der 3. und 4. Listenposition verglichen. Die 3. Listenposition spiegelt der oben angeführten Annahme zufolge den PrimacyBereich des zweiten Rehearsal-Sets wider, die anderen beiden den Recency-Bereich des ersten (2. Position) und zweiten (4. Position) Rehearsal-Sets. Ab der 5. Listenposition trat der Gesamt-Recency-Effekt der Liste in Kraft, so dass die organisierenden Einflüsse der kumulativen Rehearsal-Aktivität an Bedeutung verloren. Die Berechnung erfolgte als Test über Differenzen von Differenzen, so genannte Interaktionskontraste (Hager \& Hasselhorn, 1995).

Für den ersten Messzeitpunkt ergab sich bei einer mittleren Gruppengröße von $n=38$ $(\mathrm{N}=76)$ und einer Fehlerquadratsumme von $M S_{\mathrm{e}}=.0809$ ein $t_{\text {krit }}(.05,76)=1.665$. Die Berechnung für die Differenzunterschiede zwischen "Non-cumulative"- und "Dominantcumualtive"-Rehearsern bei den Positionen 2 und $3(t=-.24, p=.59)$ bzw. 3 und $4(t=1.63$, $p=.054)$ verfehlten die Signifikanz. Für den zweiten Messzeitpunkt zeigte sich bei $t_{\text {krit }}(.05$, 61)=1.67 und $M S_{\mathrm{e}}=.06325$ lediglich im Vergleich von 3. und 4. Position ein Unterschied zwischen den beiden Strategengruppen $(t=3.10, p<\alpha)$. Für die Differenz zwischen der Reproduktionswahrscheinlichkeit der 2. und 3. Itemposition fanden sich keine statistisch bedeutsamen Unterschiede $(t=1.33, p=.094)$. Dasselbe Muster war auch beim darauf folgenden Messzeitpunkt zu verzeichnen: $t_{\text {krit }}(.05,66)=1.669, M S_{\mathrm{e}}=.0844, t_{2-3}=.40(p=.35), t_{3-4}=1.80(p<\alpha)$. Für den vierten Messzeitpunkt fanden sich bei $t_{\text {krit }}\left(.05,64\right.$, einseitig) $=1.673, M S_{\mathrm{e}}=.07415$ keine signifikanten Unterschiede bei den beiden Vergleichen: $t_{2-3}=-.315(p=.62)$ und $t_{3-4}=1.34$ $(p=.093)$.

Dies Ergebnismuster zeigte, dass sich die durch das Rehearsal initiierten Organisationsprozesse in der Abrufphase nicht in unterschiedlichen Differenzen der Reproduktionswahrscheinlichkeiten zwischen der zweiter und dritter Listenposition im Vergleich von "Noncumulative"- und "Dominant-cumulative"-Rehearsern niederschlugen. Allerdings fanden sich diese Unterschiede beim zweiten und dritten Messzeitpunkt im Vergleich von dritter und vierter Itemposition. Es ist möglich, dass die kumulativen Memorierprozesse am Ende der zweiten Klasse noch nicht zu einem entsprechenden Recall-Vorteil durch Strukturierungsprozesse bei den „Dominant-cumulative“-Rehearsern führten und dass ein solcher Vorteil, der während der dritten Klassenstufe bestand, ein Jahr später erlosch, wenn auch vormals weniger 
strategisch vorgehende Kinder diese Organisationsprozesse nutzten. Weiterhin schien sich die Überlegenheit nur bei den mittleren Listenitems zu zeigen, wohingegen das Fehlen bedeutsamer Interaktionskontraste bei den Listenpositionen 2 und 3 auf die Existenz von allgemeinen Organisationsprozessen zu Beginn der Liste bzw. auf verdeckte Memorierprozesse von „Noncumulative"-Rehearsern hindeuten.

Tabelle 5.9: Ausmaß an subjektiver Organisation (Mittelwerte und Streuungen) in der Gesamtgruppe und getrennt nach Rehearsal-Typen zu den vier Messzeitpunkten ( $\mathrm{t}$ ).

\begin{tabular}{|c|c|c|c|c|}
\hline & Gesamtgruppe & $\begin{array}{c}\text { „Non- } \\
\text { cumumlatives“ }\end{array}$ & „Inconsistens“ & $\begin{array}{c}\text { „Dominant- } \\
\text { cumulatives“ }\end{array}$ \\
\hline $\mathrm{t}_{1}$ & $.165(.081)$ & $.163(.084)$ & $.156(.082)$ & $.176(.069)$ \\
\hline $\mathrm{t}_{2}$ & $.185(.089)$ & $.166(.081)$ & $.206(.081)$ & $.226(.11)$ \\
\hline $\mathrm{t}_{3}$ & $.188(.096)$ & $.165(.079)$ & $.235(.12)$ & $.203(.10)$ \\
\hline $\mathrm{t}_{4}$ & $.239(.098)$ & $.200(.065)$ & $.250(.10)$ & $.275(.11)$ \\
\hline
\end{tabular}

Um zu prüfen, ob die Organisationsprozesse, die zu dem geschilderten Muster führten, auf einer Nutzung der seriellen Präsentationsabfolge als Abrufhilfe beruhten, wurde das Ausmaß an subjektiver Organisation (SO) berechnet (vgl. Hasselhorn, Richter \& Lingen, submitted). Tabelle 5.9 enthält die Angaben der subjektiven Organisation getrennt für die Gesamtgruppe und für die drei Rehearsal-Typen.

Eine erste Inspektion der Daten ergab keine relevanten Unterschiede im Ausmaß der subjektiven Organisation zwischen den Lernlisten eines Messzeitpunktes $\left(F_{1}(2,158)=.80\right.$; $F_{2}(2,158)=.68 ; F_{3}(2,158)=1.65 ; F_{4}(2,158)=.81$, alle $\left.p \mathrm{~s} \geq .20\right)$. Die varianzanalytische Überprüfung der Entwicklung der subjektiven Organisation zeigte für die Gesamtgruppe einen statistisch bedeutsamen Anstieg $(F(3,237)=12.43, p<\alpha)$. Nachgeschaltete einseitige $t$-Test verdeutlichen, dass der größte Sprung in der Nutzung der seriellen Listenabfolge als Abrufhilfe zwischen dem dritten und vierten Messzeitpunkt stattgefunden hatte $(t(79)=4.11, p<\alpha)$. Die Steigerung zwischen dem ersten und zweiten Messzeitpunkt war mit $t(79)=1.74(p<\alpha)$ ebenfalls signifikant, die zwischen dem zweiten und dritten Messzeitpunkt mit $t(79)=.23(p=.41)$ nicht signifikant.

Die Überprüfung des Zusammenhangs von Rehearsal-Set-Größe und subjektiver Organisation (Tabelle 5.10) ergab analog zum Zusammenhang von Set-Größe und Reproduktionsleistung (s.o. Tabelle 5.4) beim ersten und dritten Messzeitpunkt keine statistisch bedeutsamen Korrelationen. Beim zweiten und vierten Messzeitpunkt konnten jedoch mit $r=.40$ und 
$r=.36$ signifikante Korrelationen verzeichnet werden. Der Einfluss von subjektiver Organisation auf die Reproduktionsleistung nahm über die vier Messzeitpunkte kontinuierlich zu (Tabelle 5.10). Während am Ende des zweiten Schuljahres mit $r=.19$ eine sehr geringe Korrelation zwischen den beiden Variablen vorlag, stieg dieser Zusammenhang zu Beginn des dritten Schuljahres auf $r=21$. Jedoch erst beim dritten und vierten Messzeitpunkt konnten mit $r=.28$ bzw. $r=.34$ statistisch bedeutsame Zusammenhänge nachgewiesen werden.

Tabelle 5.10: Nichtparametrische Korrelation (Spearman's rho) zwischen subjektiver Organisation (SO) und Rehearsal-Set-Größe bzw.

Reproduktionsleistung, getrennt nach Messzeitpunkten ( $\mathrm{t}$ ).

\begin{tabular}{|c|c|c|}
\hline & SO/Set-Größe & SO/Reproduktion \\
\hline $\mathrm{t}_{1}$ & .12 & .19 \\
\hline $\mathrm{t}_{2}$ & $.40^{* *}$ & .21 \\
\hline $\mathrm{t}_{3}$ & .15 & $.28^{*}$ \\
\hline $\mathrm{t}_{4}$ & $.36^{* *}$ & $.34^{* *}$ \\
\hline
\end{tabular}

$*: p<\alpha=.05 ; * *: p<\alpha=.01$

In einem weiteren Schritt wurden die drei Gruppen der Rehearsal-Typen hinsichtlich ihres Maßes an subjektiver Organisation mittels paarweiser $t$-Tests verglichen. Für den ersten Messzeitpunkt zeigten sich keine bedeutsamen Unterschiede zwischen den drei Gruppen $(t \mathrm{~s} \leq .64, p \mathrm{~s} \geq .64)$. Auch beim zweiten Messzeitpunkt unterschieden sich die Gruppen von Rehearsal-Typen nicht in ihrem Ausmaß an subjektiver Organisation ( $t \mathrm{~s} \leq 2.25, p \mathrm{~s} \geq .028)$. Allerdings erwies sich bei Messzeitpunkt 3 die Differenz zwischen den Kindern der „Noncumulative“- und der ,Inconsistent“-Gruppe mit $t(53)=2.47(p=.017)$ als signifikant; die anderen Gruppenvergleiche verfehlten dagegen das Signifikanzniveau ( $t \mathrm{~s} \leq 1.73, p \mathrm{~s} \geq .09)$. Dies war auch der einzige Messzeitpunkt, an dem die Gruppe der „Inconsistent“-Rehearser den höchsten Wert für die subjektive Organisation hatte. Beim vierten Messzeitpunkt lag mit $t(63)=$ $3.33(p<.01)$ ein signifikanter $t$-Wert für den Vergleich von „Non-cumulative“- und „Dominant-cumulative“-Rehearsern vor. Der Unterschied zwischen „Non-cumulatives“ und „Inconsistents“ verfehlte die Signifikanz knapp $(t(47)=2.05, p=.045)$ und auch zwischen der Gruppe der „Inconsistent“- und der der „Dominant-cumulative“-Rehearser bestand kein statistisch bedeutsamer Unterschied $(t(44)=.74, p=.46)$.

Eine weitere Frage war, ob elaboriertere Memorierstrategien (d.h. größere RehearsalSets) durch die größere Assoziativität zwischen Items zu geringeren Latenzzeiten in der Ab- 
rufphase führen würden. In Tabelle 5.11 sind die mittleren Inter-Response-Zeiten und deren Streuungen für die vier Messzeitpunkte angegeben. Die Darstellung erfolgt wiederum sowohl für die Gesamtgruppe als auch getrennt für die drei Rehearsal-Typ-Gruppen.

Tabelle 5.11: Inter-Response-Zeiten (Mittelwerte und Standardabweichungen in Sekunden) - Längsschnittgruppe (Gesamt und nach Rehearsal-Typen), getrennt nach Messzeitpunkten ( $\mathrm{t}$ ).

\begin{tabular}{|c|c|c|c|c|}
\hline & Gesamtgruppe & $\begin{array}{c}\text { „Non- } \\
\text { cumulatives“ }\end{array}$ & "Inconsistens“" & $\begin{array}{c}\text { „Dominant- } \\
\text { cumulatives“ }\end{array}$ \\
\hline $\mathrm{t}_{1}$ & $3.93(1.88)$ & $4.06(2.0)$ & $3.45(.82)$ & $3.38(1.16)$ \\
\hline $\mathrm{t}_{2}$ & $4.06(1.70)$ & $4.29(1.81)$ & $4.18(1.48)$ & $3.10(1.28)$ \\
\hline $\mathrm{t}_{3}$ & $3.67(1.57)$ & $3.75(1.63)$ & $4.30(1.78)$ & $3.23(1.24)$ \\
\hline $\mathrm{t}_{4}$ & $3.58(1.53)$ & $3.68(1.52)$ & $3.58(1.36)$ & $3.46(1.65)$ \\
\hline
\end{tabular}

Die inferenzstatistische Überprüfung der Entwicklung der Latenzzeiten erbrachte mit $F(3,228)=1.68(p=.17)$ keine Signifikanz. Mittelwertsvergleiche zwischen aufeinander folgenden Messzeitpunkten zeigten, dass es keine bedeutsamen Unterschiede gab ( $t \mathrm{~s} \leq 1.71, p \mathrm{~s} \geq .09)$.

Tabelle 5.12: Nichtparametrische Korrelation (Spearman's rho) zwischen der Latenz bis zur Nennung des ersten Items und der Set-Größe sowie der Reproduktionsleistung, getrennt nach Messzeitpunkten $(\mathrm{t})$.

\begin{tabular}{|c|c|c|}
\hline & Latenz/Set-Größe & Latenz/Reproduktion \\
\hline $\mathrm{t}_{1}$ & -.11 & -.13 \\
\hline $\mathrm{t}_{2}$ & -.10 & -.09 \\
\hline $\mathrm{t}_{3}$ & -.08 & -.12 \\
\hline $\mathrm{t}_{4}$ & .03 & .003 \\
\hline
\end{tabular}

Wie bereits in früheren Arbeiten fanden sich keine außergewöhnlichen Zusammenhänge zwischen Rehearsal oder Reproduktionsleistung und der Zeit bis zur Nennung des ersten Items (Tabelle 5.12; vgl. Nairne, 2002). Dieser Befund spricht erneut gegen RehearsalProzesse am Anfang der Abrufphase bzw. direkt vor Beginn der Reproduktion (vgl. Abschnitt 2.1.3). Da die Gruppe der „Inconsistent“-Rehearser zu Beginn der Untersuchung sehr klein war und es zu keinem Zeitpunkt einen statistisch relevanten Unterschied zwischen ihnen und der Gruppe der „Non-cumulative“-Rehearser gab ( $t \mathbf{s} \leq 1.04, p s \geq .30)$, wurden diese beiden 
Gruppen für die Analyse der Gruppenunterschiede zusammengefügt. Zu Beginn des Untersuchungszeitraums fanden sich mittels einseitigem t-Test keine Differenzen zwischen den beiden gebildeten Gruppen $(t(78)=1.12, p=.13)$, wohingegen die Differenz zwischen den „Dominant-cumulative“ und der Restgruppe beim zweiten und dritten Messzeitpunkt statistisch bedeutsam wurde $(t \mathrm{~s} \geq 1.73, p s<\alpha)$. Dieser Unterschied verschwand jedoch beim vierten Messzeitpunkten wieder $(t(75)=.52, p=.30)$.

Eine abschließende Frage betraf die Unterschiedlichkeit der Interitemlatenzen von Wörtern, die zusammen in ein kumulatives Rehearsal-Set eingebunden waren (,Within-Set“Latenzen), gegenüber Wörtern, die in unterschiedlichen Funktionseinheiten memoriert worden waren („Between-Set“-Latenzen) (response bursting, Pollio, Richards \& Lucas, 1969). Um zu untersuchen, ob aus der Nutzung kumulativen Rehearsals kürzere Latenzzeiten resultieren, wurden die entsprechenden Inter-Response-Zeiten der Kinder, die kumulatives Rehearsal angewandt hatten, darauf untersucht, ob sich sowohl „Within-Set“- als auch „BetweenSet"-Latenzen in der Reproduktionsphase finden ließen, die in einem zweiten Schritt miteinander verglichen wurden.

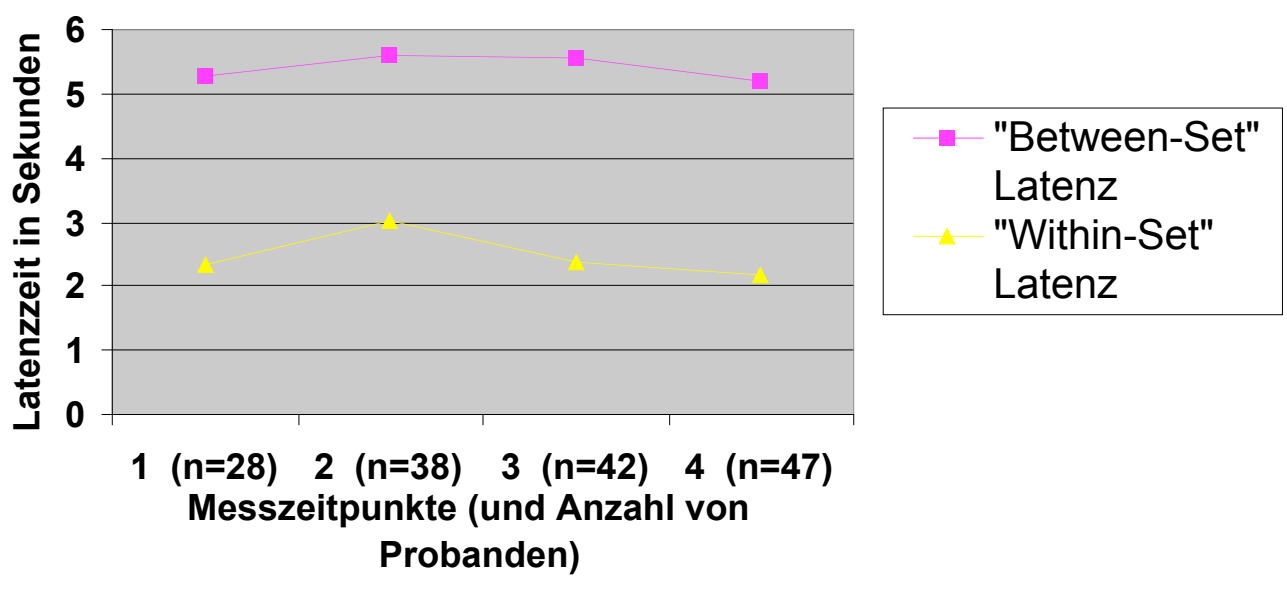

Abbildung 5.7: gepaarte „Within-Set“- und „Between-Set“-Latenzen, getrennt nach Messzeitpunkten.

Der Vergleich der gepaarten Werte für die „Within-Set“- und die „Between-Set“Latenzen (Abbildung 5.7) zeigte, dass die Inter-Response-Zeiten für Wörter innerhalb von kumulativen Rehearsal-Sets bei allen Messzeitpunkten deutlich unter denen für Wörter aus unterschiedlichen Sets und unter der mittleren Latenzzeit lagen (vgl. Tabelle 5.11). Die inferenzstatistische Überprüfung der Differenzen ergab mit $t \mathrm{~s} \geq 2.98(p \mathrm{~s}<\alpha)$ für alle Messzeitpunkte signifikante Werte. Diese deutliche Diskrepanz zwischen den beiden Latenzformen weist darauf hin, dass durch die Konklusion von Items während kumulativer Memorierpro- 
zesse eine assoziative Verknüpfung zwischen den Items aufgebaut wurde, die sich in einer entsprechenden Geschwindigkeit der Abrufprozesse dieser Items äußerte. Für Items aus unterschiedlichen Sets war keine solche enge Verknüpfung zu erwarten, was sich in deutlich längeren Inter-Response-Zeiten niederschlägt.

Auf der Betrachtungsebene der intraindividuellen Entwicklungsverläufe konnte gezeigt werden, dass die Entdeckung und der verstärkte Gebrauch von kumulativem Rehearsal sich wesentlich zwischen zweiter und dritter Klasse sowie im Verlauf des dritten Schuljahres vollzog. Sowohl die deskriptive Betrachtung als auch der Chi-Quadrat-Test nach McNemar sprechen hier für einen ersten Entwicklungsschub zwischen dem Ende der zweiten und dem Anfang der dritten Klasse, der zur Entdeckung des kumulativen Rehearsals als Lernstrategie führt. In einem zweiten Entwicklungsschub während der dritten Klassenstufe kommt es zu einem Anstieg im Ausmaß der Nutzung kumulativen Rehearsals. Ein damit einhergehender Leistungsvorteil wurde allerdings erst beim vierten Messzeitpunkt erzielt. Die Verlaufsdaten wiesen eine gewisse Stabilität und einen generellen Trend zu einem elaborierteren strategischen Verhalten im Verlauf der späten Grundschuljahre auf. Es fanden sich keine Belege für einen abrupten Prozess von Strategieaneignung und -verlust (Achterbahn-Phänomen).

Die Reproduktionskurven aller Rehearsal-Typ-Gruppen wiesen mehrfach U-förmige Verläufe auf. Diese Verlaufsstruktur kann als Hinweis für Organisationsprozesse in der Abrufphase gewertet werden. Kinder, die kumulatives Rehearsal praktiziert hatten, nutzten ab dem Ende der dritten Klasse verstärkt die serielle Präsentationsreihenfolge als Abrufhilfe. In der dritten Klasse konnten auch Belege für Unterschiede in den Inter-Response-Zeiten zwischen den „Dominant-cumulative“-Rehearsern und der Restgruppe von Rehearsal-Typen entdeckt werden. Für kumulative Rehearser zeigte sich weiterhin das Phänomen des response bursting mit signifikant niedrigeren Interitemlatenzen zwischen Wörtern aus einem (kumulativen) Rehearsal-Set im Vergleich zu Itemlatenzen zwischen Wörtern aus unterschiedlichen Rehearsal-Sets.

\subsection{Die Bedeutung metakognitiver und kapazitiver Aspekte - Über- prüfung der Vorhersagen der Strategie-Emergenz-Theorie}

\subsubsection{Psychologische Hypothesen und Vorhersagen}

Wenn man, ausgehend von der Vermutung über die Entwicklungsparallelität von Organisations- und Rehearsalprozessen (Hasselhorn \& Lindner-Müller, 1995), die Annahmen der Strategie-Emergenz-Theorie (Hasselhorn, 1996) auf die Entwicklung von kumulativem Re- 
hearsal anwendet, so ist die zentrale Aussage, dass es im Alter zwischen 9 und 10 Jahren zu einem deutlichen Anstieg an metakognitivem Wissen über den Nutzen und die Einsatzmöglichkeiten kumulativer Rehearsal-Prozesse und zeitgleich zu ihrer verstärkten Verwendung bei der Bearbeitung von episodischen Gedächtnisaufgaben kommen sollte. Bei älteren Kindern sollte sich ein deutlicher Zusammenhang zwischen diesen beiden Faktoren und ebenfalls ein Leistungsgewinn aus der Anwendung des kumulativen Rehearsals finden lassen ( $\left.\mathrm{PH}_{3}\right)$. Dagegen sollte der Einfluss kapazitiver Aspekte auf die Gedächtnisleistung im Verlauf der späten Grundschuljahre zurückgehen $\left(\mathrm{PH}_{4}\right)$. Für die im folgenden Kapitel dargestellten Analysen zur Überprüfung dieser Annahmen können daraus fünf psychologische Vorhersagen abgeleitet werden.

- Bei zehnjährigen Kindern existiert ein ausgeprägteres metamemoriales Wissen für Rehearsal-Prozesse als bei neunjährigen Kindern $\left(\mathrm{PV}_{3,1}\right)$.

- Parallel dazu steigt der Prozentsatz an Kindern, die als „Dominant-cumulative“Rehearser bzw. als Rehearsal-Strategen klassifiziert werden können ( $\left.\mathrm{PV}_{3,2}\right)$.

- Bei den älteren Kindern, aber noch nicht bei den jüngeren existiert ein positiver Zusammenhang zwischen dem aufgabenspezifischen Metagedächtnis und dem strategischen Verhalten $\left(\mathrm{PV}_{3,3}\right)$.

- Das kumulative Rehearsal führt (vor allem bei älteren Kindern) zu besseren Reproduktionsleistungen, d.h., das Rehearsal-Strategen eine größere Behaltensleistung haben als Nichtstrategen und dass der Zusammenhang von Set-Größe und Reproduktionsleistung bei Zehnjährigen ausgeprägter ist als bei Neunjährigen( $\left(\mathrm{PV}_{3,4}\right)$.

- Im Verlauf der späten Grundschuljahre nimmt der korrelative Zusammenhang zwischen der Zahlenspanne und der Reproduktionsleistung ab ( $\left.\mathrm{PV}_{4,1}\right)$.

\subsubsection{Befunde}

Die erste psychologische Vorhersage besagt, dass bei älteren Kindern ein ausgeprägteres aufgabenspezifisches Metagedächtnis vorliegen soll. In Tabelle 5.13 sind die durchschnittlichen Werte und Standardabweichungen in der Metagedächtnisskala für die Longitudinalgruppe bei Messzeitpunkt 3 und 4 dargestellt. Der maximal erreichbare Wert lag bei neun Punkten. Es konnte mit $t(79)=3.06(p<\alpha)$ eine statistisch bedeutsame Steigerung im metamemorialen Wissen über Rehearsal-Prozesse zwischen Ende der dritten und Anfang der vierten Klasse auf Gruppenebene verzeichnet werden. 
Tabelle 5.13 Mittelwerte (und Standardabweichungen) der Metagedächtnisskala bei Messzeitpunkt $3\left(\mathrm{t}_{3}\right)$ und $4\left(\mathrm{t}_{4}\right)$ - Longitudinalgruppe.

\begin{tabular}{|c|c|}
\hline & Metamemoriales Wissen \\
\hline $\mathrm{t}_{3}$ & $3.19(2.18)$ \\
\hline $\mathrm{t}_{4}$ & $4.08(2.12)$ \\
\hline
\end{tabular}

Es zeigte sich, dass die signifikante Zunahme an strategiespezifischem Metagedächtnis auf Gruppenebene vor allem auf die Kinder zurückgeführt werden konnte, die zum vierten Messzeitpunkt erstmals als „Dominant-cumulative“-Rehearser klassifiziert worden waren. Die Kinder dieser Teilgruppe $(n=10)$ erzielten beim dritten Messzeitpunkt einen durchschnittlichen Wert im strategiespezifischen Metagedächtnis von $M=2.5(S D=1.65)$ und konnten diesen zum vierten Messzeitpunkt auf $M=4.4(S D=2.07)$ steigern. Diese Differenz ist mit $t(9)=2.75(p<\alpha)$ statistisch bedeutsam. Dagegen verzeichneten die Kinder, die zu keinem der beiden Messzeitpunkte als „Dominant-cumulative“ klassifiziert wurden ( $\mathrm{n}=45)$, keine signifikante Steigerung $(t(44)=1.5, p=.07)$. Der Mittelwert der konsistent als „Dominantcumulative"-Rehearser klassifizierten Kinder $(\mathrm{n}=21)$ betrug beim dritten Messzeitpunkt $M=3.43(S D=2.67)$ und beim vierten Messzeitpunkt $M=4.52$ ( $S D=1.94)$. Auch hier konnte mit $t(20)=1.71(p=.051)$ keine bedeutsame Zunahme im strategiespezifischen Metagedächtnis verzeichnet werden. Die kleinste Gruppe bildeten die Kinder, die ihren Status als „Dominantcumulative"-Rehearser vom dritten auf den vierten Messzeitpunkt einbüßten ( $n=4)$. Sie konnten ihren Durchschnittswert im strategiespezifischen Metagedächtnis zwar von $M=5.5$ $(S D=2.08)$ beim dritten Messzeitpunkt auf $M=6.25(S D=2.75)$ ein halbes Jahr später verbessern, was in beiden Fällen den höchsten Wert einer Teilgruppe darstellt. Die Differenz blieb jedoch mit $t(3)=.57(p=.30)$ unter der Signifikanzgrenze.

Wie bereits im letzten Abschnitt dargestellt stieg der Anteil an Kindern, die als „Dominant-cumulative“-Rehearser klassifiziert wurden, von 25 (31\%) beim dritten Messzeitpunkt auf 31 (39\%) ein halbes Jahr später. Damit kann die zweite psychologische Vorhersage zwar auf der deskriptiven Ebene und bei einem moderaten Strategenkriterium als bestätigt gelten, der gefundene Unterschied erwies sich allerdings nicht als bedeutsam (McNemar-Test, $p>\alpha$ ).

Wird analog zu dem Vorgehen von Hasselhorn und Lindner-Müller (1995) ein Doppelkriterium gewählt, so kann ein Rehearsalstratege als ein Kind definiert werden, das a) eine Rehearsal-Set-Größe $\geq 2.25$ Items hat und b) bei der Nachbefragung spontan angibt, beim Lernen immer mehrere Items zusammen memoriert zu haben. Dieses strenge Kriterium für die Strategenklassifikation erfüllten beim dritten Messzeitpunkt 20 (25\%) und beim vierten 
Messzeitpunkt 28 (35\%) der 80 Kinder in der Longitudinalgruppe. Der gefundene Unterschied erwies sich ebenfalls als statistisch nicht bedeutsam (McNemar-Test, $p>\alpha$ ). Bei einem strengeren Strategenkriterium fand sich also auch kein signifikanter Anstieg in dem Anteil der als Strategen klassifizierten Kinder beim Wechsel von der dritten zur vierten Klasse.

Entsprechend der dritten aus der Strategie-Emergenz-Theorie abgeleiteten psychologischen Vorhersage soll das von der dritten zur vierten Klassenstufe zunehmende Metagedächtnis zentrale Ursache für das strategischere Verhalten der älteren Kinder sein. Dies sollte sich in einem größeren Zusammenhang zwischen dem Metagedächtnis und dem strategischen Verhalten bei älteren gegenüber jüngeren Kindern zeigen. Zur Überprüfung dieser Annahme wurden die korrelativen Zusammenhänge zwischen dem erfasstem Metagedächtnis für Rehearsal und der durchschnittlichen Set-Größe berechnet (Tabelle 5.14).

Tabelle 5.14: Spearman's Korrelationskoeffizienten zwischen der Rehearsal-Set-Größe und dem strategiespezifischen Metagedächtnis bei Messzeitpunkt $3\left(t_{3}\right)$ und $4\left(t_{4}\right)$.

\begin{tabular}{|l|c|c|}
\hline & $\begin{array}{c}\text { Rehearsal-Set-Größe } t_{3} \\
\text { (3. Klasse) }\end{array}$ & $\begin{array}{c}\text { Rehearsal-Set-Größe } t_{4} \\
\text { (4. Klasse) }\end{array}$ \\
\hline $\begin{array}{l}\text { strategiespezifisches Me- } \\
\text { tagedächtnis } t_{3}\end{array}$ & .12 & .003 \\
\hline $\begin{array}{l}\text { strategiespezifisches Me- } \\
\text { tagedächtnis } t_{4}\end{array}$ & .10 & .17 \\
\hline
\end{tabular}

Zu keinem der beiden Messzeitpunkte konnte ein signifikanter Zusammenhang zwischen dem Metagedächtnis über Rehearsal und dem entsprechenden Verhalten in der Lernphase nachgewiesen werden. Es scheint, dass das aufgabenspezifische Metagedächtnis auf keiner der beiden Altersstufen als zentraler interner Faktor des strategischen Verhaltens in der verwendeten seriellen Lernaufgabe fungierte. Allerdings konnten differenzierte Analysen belegen, dass der Zusammenhang zwischen aufgabenspezifischem Metagedächtnis und der durchschnittlichen Set-Größe beim letzten Messzeitpunkt für die Gruppe der „Dominantcumulative“-Rehearser mit $r=.24$ rein deskriptiv deutlich stärker ausfiel als bei den nicht „Dominant-cumulative“-Rehearsern mit $r=.07$. Ein halbes Jahr zuvor lag der Korrelationskoeffizient für die „Dominant-cumulatives“ noch bei $r=.02$, der für die Kinder, die nicht als „Dominant-cumulative“ klassifiziert worden waren, bei $r=.06$. Die Überprüfung der Differenzen ergab keine signifikanten Unterschiede in den Korrelationskoeffizienten $(t s \leq .73, p s \geq .43)$.

Die vierte zu prüfende Annahme aus der Strategie-Emergenz-Theorie bezieht sich auf die positiven Leistungskonsequenzen des kumulativen Memorierverhaltens älterer Kinder. 
Wie bereits weiter oben ausgeführt (s.o. Abschnitt 5.1, Tabelle 5.1) reproduzierten die Kinder der Longitudinalgruppe beim dritten Messzeitpunkt im Mittel $M=5.85$ ( $S D=1.49$ ) Items. Der Mittelwert für den vierten Messzeitpunkt betrug $M=6.31(S D=1.39)$. Der $t$-Test für gepaarte Stichproben bestätigte die Erwartung, dass Viertklässer den Drittklässlern in der durchschnittlichen Reproduktionsleistung bedeutsam überlegen waren $(t(79)=2.97, p<\alpha)$. Auch der $\mathrm{Zu}-$ sammenhang zwischen der Strategievariablen (durchschnittliche Set-Größe) und der Leistung fiel mit $r_{4}=.28$ beim vierten Erhebungszeitpunkt höher aus als ein halbes Jahr zuvor $\left(r_{3}=.14\right)$.

Eine letzte Prüfung galt der Frage des Zusammenhangs zwischen Strategenstatus (strenges Kriterium) und Reproduktionsleistung. Für den dritten Messzeitpunkt konnte mit $t(78)=1.92(p<\alpha)$ eine Überlegenheit der Strategen gegenüber den Nichtstrategen verzeichnet werden (einseitiger t-Test). Bei Messzeitpunkt 4 reproduzierten die Nichtstrategen $(n=52)$ im Mittel $M=6.01(S D=1.25)$ Items. Der Mittelwert der in der Longitudinalgruppe gefundenen Strategen $(\mathrm{n}=28)$ lag bei $M=6.87(S D=1.08)$. Diese Differenz erwies sich ebenfalls als signifikant $(t(78)=2.76, p<\alpha)$. Bei einem strengen Kriterium findet sich also bereits in der dritten Klassenstufe eine Überlegenheit der Rehearsal-Strategen gegenüber den Nichtstrategen (vgl. 5.3.2). Der gefundene Effekt fällt aber auf der vierten Klassenstufe deutlich stärker aus.

Trotz dieses letzten statistisch bedeutsamen Ergebnisses stellt sich angesichts des uneinheitlichen Befundmusters die Frage, ob die von Hasselhorn (1996) in der StrategieEmergenz-Theorie formulierte Annahme über den Zusammenhang von alterskorrelierter Zunahme des strategiespezifischem Metagedächtnisses und daraus resultierender Leistungskonsequenz für die Strategie des kumulativen Rehearsals zutreffend ist. Die aus der StrategieEmergenz-Theorie abgeleiteten Vorhersagen können durch die berichteten Ergebnisse nicht in vollem Umfang bestätigt werden.

Als eine alternative Interpretation bietet sich die Annahme eines großen Einflusses der ebenfalls alterskorrelierten funktionalen Arbeitsgedächtniskapazität auf die Entwicklung der Rehearsal-Strategie und der Lernleistung an. Diese spielen nach Hasselhorn (1996, S. 81) „bei der Entwicklung kategorialen Organisierens eine eher marginale Rolle“, da sie sowohl automatische als auch strategische Prozesse begünstigen. Die funktionale Kapazität des Arbeitsgedächtnisses ist jedoch entscheidend für die Anzahl von Items, die memoriert werden können.

Sowohl für die Zahlenspanne vorwärts $(F(3,237)=12.14, p<\alpha)$ als auch für die Zahlenspanne rückwärts $(F(3,237)=6.11, p<\alpha)$ konnte auf der Gruppenebene ein signifikanter Anstieg von Ende der zweiten bis Anfang der vierten Klasse verzeichnet werden (Tabelle 5.15). 
Tabelle 5.15: Mittelwerte und Standardabweichung der Zahlenspanne vorwärts und rückwärts zu den vier Messzeitpunkten ( $\mathrm{t}$ ) - Longitudinalgruppe.

\begin{tabular}{|c|c|c|}
\hline & Zahlenspanne vorwärts & Zahlenspanne rückwärts \\
\hline $\mathrm{t}_{1}$ & $4.87(.84)$ & $3.75(.68)$ \\
\hline $\mathrm{t}_{2}$ & $5.13(.88)$ & $3.89(.72)$ \\
\hline $\mathrm{t}_{3}$ & $5.34(.99)$ & $4.04(.79)$ \\
\hline $\mathrm{t}_{4}$ & $5.52(1.20)$ & $4.19(1.07)$ \\
\hline
\end{tabular}

Anschließende $t$-Tests zwischen aufeinander folgenden Messzeitpunkten belegten nur für die Zahlenspanne vorwärts zwischen dem ersten und zweiten $(t(79)=3.03, p<\alpha)$ sowie dem zweiten und dritten Messzeitpunkt $(t(79)=2.17, p<\alpha)$ signifikante Zuwächse. Alle anderen Differenzen zwischen benachbarten Messzeitpunkten erwiesen sich als nicht statistisch bedeutsam ( $t \mathrm{~s} \leq 1.54, p \mathrm{~s} \geq .13)$. Der Anstieg der Standardabweichung, der in beiden Zahlenspannenmaßen verzeichnet werden konnte, spiegelt allerdings die zunehmende Variabilität in der Entwicklung der funktionalen Gedächtniskapazität im Verlauf der späteren Grundschuljahre wider.

Tabelle 5.16: Zusammenhang zwischen der Zahlenspanne vorwärts (ZSV) und rückwärts (ZSR) und der Lernleistung sowie Set-Größe - Longitudinalgruppe.

\begin{tabular}{|l|c|c|c|c|}
\hline & $\mathrm{ZSV}_{1} / \mathrm{ZSR}_{1}$ & $\mathrm{ZSV}_{2} / \mathrm{ZSR}_{2}$ & $\mathrm{ZSV}_{3} / \mathrm{ZSR}_{3}$ & $\mathrm{ZSV}_{4} / \mathrm{ZSR}_{4}$ \\
\hline Recall $_{1}$ & $.04 / .22$ & & & \\
\hline Recall $_{2}$ & & $.26^{*} / .28^{*}$ & & \\
\hline Recall $_{3}$ & & & $.15 / .31^{*}$ & \\
\hline Recall $_{4}$ & & & & $.18 / .23^{*}$ \\
\hline
\end{tabular}

\begin{tabular}{|c|c|c|c|c|}
\hline & $\mathrm{ZSV}_{1} / \mathrm{ZSR}_{1}$ & $\mathrm{ZSV}_{2} / \mathrm{ZSR}_{2}$ & $\mathrm{ZSV}_{3} / \mathrm{ZSR}_{3}$ & $\mathrm{ZSV}_{4} / \mathrm{ZSR}_{4}$ \\
\hline Set-Größe $_{1}$ & $.05 / .03$ & & & \\
\hline Set-Größe $_{2}$ & & $.13 / .24^{*}$ & & \\
\hline Set-Größe $_{3}$ & & & $.19 / .22^{*}$ & \\
\hline Set-Größe & & & & $.24^{*} / .27^{*}$ \\
\hline
\end{tabular}

Die Berechnung der Zusammenhänge zwischen der Zahlenspanne vorwärts/rückwärts und der Reproduktionsleistung bzw. der Rehearsal-Set-Größe (Tabelle 5.16) zeigten beim ersten Messzeitpunkt keine bedeutsame Korrelation zwischen der Zahlenspanne rückwärts auf der einen und der Lernleistung sowie der durchschnittlichen Rehearsal-Set-Größe auf der anderen Seite. Erst beim zweiten und dritten Messzeitpunkt (Klasse 3) schien die Arbeitsgedächtniskapazität einen substantiellen Einfluss zu haben, der zumindest beim Zusammenhang 
von Lernleistung und Zahlenspanne rückwärts beim vierten Erhebungszeitpunkt (Klasse 4) wieder deutlich zurückging Dies kann im Sinne der Strategie-Emergenz-Theorie als Hinweis darauf interpretiert werden, dass die strategische Komponente zwischen 9 und 10 Jahren an Einfluss gewinnt und somit kapazitive Aspekte eher in den Hintergrund treten. Der Einfluss der Gedächtniskapazität auf die Rehearsal-Set-Größe war jedoch auch in der vierten Klassenstufe unvermindert.

Wenn man berücksichtigt, dass die Zahlenspanne vorwärts stärker mit der Artikulationsgeschwindigkeit als Indikator für die Geschwindigkeit des subvokalen RehearsalProzesses korrelierte (vgl. Abschnitt 2.3.1) und die Zahlenspanne rückwärts eher zentralexekutive Aspekte beinhaltet, ist die Diskrepanz zwischen den Korrelationswerten für die Zahlenspanne vorwärts und rückwärts ein Hinweis darauf, dass bei dem für das kumulative Rehearsal notwendigen Abrufprozess früherer Items im Sinne eines Mini-Recalls nicht so sehr die Artikulationsgeschwindigkeit, sondern die zentral-exekutiven Aspekte im Vordergrund stehen. Dies sollte sich darin zeigen, dass bei den „Dominant-cumulative“-Rehearsern, nicht aber bei den Kindern der anderen Gruppen, hohe Korrelationen bei der Zahlenspanne rückwärts und der Set-Größe sowie der daraus resultierenden Lernleistung auftreten.

Tabelle 5.17: Zusammenhang zwischen der Zahlenspanne vorwärts (ZSV) und rückwärts (ZSR) und der Lernleistung sowie Set-Größe - Nicht-,,Dominant-cumulatives“.

\begin{tabular}{|c|c|c|c|c|}
\hline & $\begin{array}{c}\mathrm{ZSV}_{1} / \mathrm{ZSR}_{1} \\
(\mathrm{n}=68)\end{array}$ & $\begin{array}{c}\mathrm{ZSV}_{2} / \mathrm{ZSR}_{2} \\
(\mathrm{n}=66)\end{array}$ & $\begin{array}{c}\mathrm{ZSV}_{3} / \mathrm{ZSR}_{3} \\
(\mathrm{n}=55)\end{array}$ & $\begin{array}{c}\mathrm{ZSV}_{4} / \mathrm{ZSR}_{4} \\
(\mathrm{n}=49)\end{array}$ \\
\hline Recall $_{1}$ & $.03 / .22$ & & & \\
\hline Recall $_{2}$ & & $.18 / .27^{*}$ & & \\
\hline Recall $_{3}$ & & & $.02 / .23$ & \\
\hline Recall $_{4}$ & & & & $-.02 /-.02$ \\
\hline
\end{tabular}

\begin{tabular}{|c|c|c|c|c|}
\hline & $\begin{array}{c}\mathrm{ZSV}_{1} / \mathrm{ZSR}_{1} \\
(\mathrm{n}=68)\end{array}$ & $\begin{array}{c}\mathrm{ZSV}_{2} / \mathrm{ZSR}_{2} \\
(\mathrm{n}=66)\end{array}$ & $\begin{array}{c}\mathrm{ZSV}_{3} / \mathrm{ZSR}_{3} \\
(\mathrm{n}=55)\end{array}$ & $\begin{array}{c}\mathrm{ZSV}_{4} / \mathrm{ZSR}_{4} \\
(\mathrm{n}=49)\end{array}$ \\
\hline Set-Größe $_{1}$ & $-.02 /-.06$ & & & \\
\hline Set-Größe $_{2}$ & & $.08 / .13$ & & \\
\hline Set-Größe $_{3}$ & & & $-.11 / .08$ & \\
\hline Set-Größe & & & & $.12 /-.02$ \\
\hline
\end{tabular}

$*: p<\alpha=.05$

Die Kinder, die nicht als „Dominant-cumulative“-Rehearser klassifiziert worden waren, zeigten bis auf eine Ausnahme keine bedeutsamen Zusammenhänge zwischen den kapazitiven Maßen und der Lernleistung bzw. der strategischen Komponente (Tabelle 5.17). Nur bei Messzeitpunkt 2 gab es eine signifikante Korrelation zwischen der Zahlenspanne rückwärts und der Reproduktionsleistung ( $r=.27)$. 
Tabelle 5.18: Zusammenhang zwischen der Zahlenspanne vorwärts (ZSV) und rückwärts (ZSR) und der Lernleistung sowie Set-Größe - „,Dominant-cumulatives“.

\begin{tabular}{|c|c|c|c|c|}
\hline & $\begin{array}{c}\mathrm{ZSV}_{1} / \mathrm{ZSR}_{1} \\
(\mathrm{n}=12)\end{array}$ & $\begin{array}{c}\mathrm{ZSV}_{2} / \mathrm{ZSR}_{2} \\
(\mathrm{n}=14)\end{array}$ & $\begin{array}{c}\mathrm{ZSV}_{3} / \mathrm{ZSR}_{3} \\
(\mathrm{n}=25)\end{array}$ & $\begin{array}{c}\mathrm{ZSV}_{4} / \mathrm{ZSR}_{4} \\
(\mathrm{n}=31)\end{array}$ \\
\hline Recall $_{1}$ & $-.08 / .02$ & & & \\
\hline Recall $_{2}$ & & $.70 * * / .22$ & & \\
\hline Recall $_{3}$ & & & $.34 / .38$ & \\
\hline Recall $_{4}$ & & & & $.39 * / .55^{* *}$ \\
\hline
\end{tabular}

\begin{tabular}{|c|c|c|c|c|}
\hline & $\begin{array}{c}\mathrm{ZSV}_{1} / \mathrm{ZSR}_{1} \\
(\mathrm{n}=12)\end{array}$ & $\begin{array}{c}\mathrm{ZSV}_{2} / \mathrm{ZSR}_{2} \\
(\mathrm{n}=14)\end{array}$ & $\begin{array}{c}\mathrm{ZSV}_{3} / \mathrm{ZSR}_{3} \\
(\mathrm{n}=25)\end{array}$ & $\begin{array}{c}\mathrm{ZSV}_{4} / \mathrm{ZSR}_{4} \\
(\mathrm{n}=31)\end{array}$ \\
\hline Set-Größe $_{1}$ & $.49 /-.01$ & & & \\
\hline Set-Größe $_{2}$ & & $.71^{* *} / .22$ & & \\
\hline Set-Größe $_{3}$ & & & $-.04 / .02$ & \\
\hline Set-Größe & & & & $.32 / .50^{* *}$ \\
\hline
\end{tabular}

$*: p<\alpha=.05 ; * *: p<\alpha=.01$

Für die Gruppe der „Dominant-cumulatives“ konnten mehrfach Zusammenhänge bedeutsamen Ausmaßes verzeichnet werden (Tabelle 5.18). Beim zweiten Erhebungszeitpunkt fanden sich signifikante Korrelationen zwischen der Zahlenspanne vorwärts und der Reproduktionsleistung sowie der Set-Größe. Ein halbes Jahr später gab es dagegen keine statistisch bedeutsamen Zusammenhänge mehr. Zum vierten Messzeitpunkt konnten vor allem hohe Korrelationen mit der Zahlenspanne rückwärts und den beiden Abhängigen Variablen SetGröße und Reproduktionsleistung verzeichnet werden.

Kumulative Rehearsal-Prozesse stellen gerade bei ihrem Erwerb kapazitätsbelastende Prozesse dar (vgl. Guttentag, 1984). Es ist nachvollziehbar, dass bei der Akquisition von kumulativem Rehearsal vor allem die Artikulationsgeschwindigkeit eine große Rolle spielt, damit eine größere Anzahl von Items innerhalb eines kurzen Zeitraums memoriert werden kann, und dass diese Fähigkeit erst durch Übung automatisiert werden muss, damit sie nur noch wenig kognitive Ressourcen verbraucht. Erst dann profitieren Kinder maximal von einer besseren Gedächtniskapazität.

Die gefundenen Daten stützen diese Sichtweise. Die signifikanten Korrelationen zur Zahlenspanne rückwärts deuten an, dass auf dem qualitativ höheren Niveau zentral-exekutive Aspekte eine bedeutende Rolle spielen, also die Fähigkeit, die Rehearsal-Sets und den dafür notwendigen Abruf aus dem Gedächtnis im Sinne des beschriebenen Mini-Recalls zu koordinieren. Hierfür ist das Hin- und Herschalten zwischen Suchräumen und der Abruf sowie die Manipulation von Informationen aus dem Langzeitgedächtnis notwendig. Der Rückgang der Korrelationen beim dritten Messzeitpunkt könnte danach durch den Hinzugewinn einer größe- 
ren Anzahl von Kindern zur Gruppe der „Dominant-cumulative“-Rehearser zustande gekommen sein, die noch nicht den vollen Gewinn aus ihrer funktionalen Kapazität erzielen konnten, da die Durchführung der kumulativen Gedächtnisstrategie sie noch zu viel mentale Ressourcen kostete.

Die inferenzstatistische Überprüfung der Differenzen in den gefundenen korrelativen Zusammenhängen zeigte für den ersten Messzeitpunkt keine signifikanten Werte $(t \mathrm{~s} \leq .57 /$ 1.57; $p \mathrm{~s} \geq .12)$. Weiter erwies sich, dass die „Dominant-cumulative“-Rehearser beim zweiten Messzeitpunkt bedeutsam höhere Korrelationen zwischen der Zahlenspanne vorwärts und der Reproduktionsleistung $(t(78)=-2.09, p<\alpha)$ bzw. der Set-Größe hatten $(t(78)=-2.47, p<\alpha)$ als die nicht „Dominant-cumulatives“. Beim dritten Messzeitpunkt fanden sich dagegen keine $t$ Werte bedeutsamen Ausmaßes ( $t \mathrm{~s} \leq .24 /-1.31, p s \geq .19)$. Für den letzten Messzeitpunkt konnten signifikante Differenzen in den Korrelationskoeffizienten der Zahlenspanne rückwärts und der Recall-Leistung $(t(78)=-2.66, p<\alpha)$ bzw. der durchschnittlichen Rehearsal-Set-Größe $(t(78)=-$ $2.38, p<\alpha)$ zwischen den beiden Gruppen verzeichnet werden.

Weiterhin ergaben sich für die beim dritten Messzeitpunkt erstmals als „Dominantcumulative"-Rehearser klassifizierten Kinder $(\mathrm{n}=13)$ keine bedeutsamen Zusammenhänge zwischen den beiden Maßen für die Gedächtniskapazität und der Reproduktionsleistung bzw. der Set-Größe sowie zwischen den beiden letzten Variablen. Dagegen konnten für die konsistent als „Dominant-cumulative“ klassifizierten Kinder $(\mathrm{n}=12)$ signifikante Korrelationen zwischen der Zahlenspanne vorwärts und rückwärts mit der Reproduktionsleistung $\left(r_{\mathrm{ZSV}}=.58\right.$; $\left.r_{\mathrm{ZSR}}=.64, p \mathrm{~s}<\alpha\right)$ sowie zwischen der Zahlenspanne rückwärts und der Set-Größe verzeichnet werden $(r=.62, p<\alpha)$. Des Weiteren fand sich für diese Teilgruppe ein enger Zusammenhang zwischen der durchschnittlichen Set-Größe und der Reproduktionsleistung ( $r=.65, p<\alpha)$.

Für diese letzte Gruppe zeigte sich damit insgesamt deutlich der angesprochene Wechsel in dem Bedarf von Verarbeitungsgeschwindigkeit beim Erlernen von kumulativen Memorierprozessen (beim zweiten Messzeitpunkt) hin zu einer stärkeren Bedeutung von Exekutivprozessen, wenn die Kinder die Nutzung des kumulativen Rehearsals automatisiert haben und damit stärker von ihrer Gedächtniskapazität profitieren (beim dritten Messzeitpunkt).

Die Überprüfung der Annahmen der Strategie-Emergenz-Theorie für den Erwerb kumulativen Rehearsals führte zu einem heterogenen Befundmuster. Zwar konnte entsprechend der Vorhersage ein Anstieg des metamemorialen Strategiewissens für Rehearsal zwischen dritter und vierter Klasse gefunden werden, es ließ sich jedoch kein relevanter Zusammenhang zwischen dem strategiespezifischen Metagedächtnis und der Rehearsal-Set-Größe nachweisen. Allerdings verzeichneten die Rehearsal-Strategen deutlichere Zuwächse in ihrem 
Ausmaß an Metakognition und ihrer Recall-Leistung als die Nichtstrategen. Sowohl für den dritten als auch für den vierten Messzeitpunkt zeigten die Rehearsal-Strategen bei einem strengen Kriterium einen Erinnerungsvorteil gegenüber den Nicht-Strategen. Für die beiden Zahlenspannenmaße konnte ein alterskorrelierter Anstieg verzeichnet werden. Das gefundene Ergebnismuster stützt die Annahme vom Einfluss kapazitiver Aspekte beim Erwerb kumulativer Memorierprozesse und die Bedeutung von Exekutivfunktionen in einem späteren Stadium der effizienten Nutzung kumulativen Rehearsals.

\subsection{Belege für eine Nutzungsineffizienz bei kumulativem Rehearsal}

\subsubsection{Psychologische Hypothese und Vorhersagen}

Bislang existieren keine empirischen Studien über das Auftreten einer Nutzungsineffizienz bei Rehearsal. Daher gibt es auch keine etablierten Kriterien oder Indikatoren, anhand derer die empirische Relevanz dieses Phänomens, bei dem ein Kind eine Gedächtnisstrategie zwar spontan, aber ohne einen entsprechenden Lerngewinn anwendet, für die Memorierstrategien entschieden werden könnte. Ausgehend von der oben vorgenommenen Strategenklassifikation und allgemeinen Definitionskriterien (Miller, 2000) soll hier ein erster Versuch einer adäquaten Beantwortung der Frage nach Belegen für eine Nutzungsineffizienz bei kumulativem Rehearsal unternommen werden. Entsprechend der allgemeinen Kriterien über das Vorliegen einer Nutzungsineffizienz sollte der erstmalige spontane Gebrauch kumulativer Rehearsal-Prozesse bzw. die hauptsächliche Nutzung kumulativen Rehearsals zumindest von keiner korrespondierenden Leistungssteigerung, vielleicht sogar von einem Leistungsrückgang begleitet sein. Auch wenn neu als Strategen klassifizierte Kinder keine bedeutsam höheren Steigerungen der Reproduktionsleistung zwischen zwei Messzeitpunkten zeigen als Nichtstrategen, kann dies als Hinweis auf eine Nutzungsineffizienz für Rehearsal interpretiert werden $\left(\mathrm{PH}_{5}\right)$. Die hieraus ableitbaren psychologischen Vorhersagen lauten:

- Der Wechsel vom „Non-cumulative“- zum Inconsistent“-Status bzw. vom „Inconsistent"- zum „Dominant-cumulative“-Status zwischen aufeinander folgenden Messzeitpunkten ist von keiner korrespondierenden Leistungssteigerung bzw. sogar von einem Leistungsrückgang begleitet $\left(\mathrm{PV}_{5,1}\right)$.

- Der Zuwachs in der Leistung zwischen zwei Messzeitpunkten ist bei „Aufsteigern/Strategieentdeckern“ nicht größer als bei ,konsistenten Nichtstrategen“ $\left(\mathrm{PV}_{5,2}\right)$.

Für die Auswertung wurden fünf Gruppen gebildet: „konsistente Nichtstrategen“, „Absteiger/Strategieverlierer“, „ineffiziente Aufsteiger/Strategieentdecker“, „,effiziente Aufstei- 
ger/Strategieentdecker“ und „konsistente Strategen“. In die Gruppe der „konsistenten Nichtstrategen“ kamen die Kinder, die bei zwei aufeinander folgenden Messzeitpunkten als „Noncumulative“-Rehearser klassifiziert worden waren. Als „Absteiger/Strategieverlierer“ wurden die Kinder eingeordnet, die von einem höheren Strategenstatus („Dominant-cumulative“ oder „Inconsistent“) auf einen niedrigeren Status („Inconsistent“ bzw. „Non-cumulative“) zurückfielen. Wenn Kinder bei einem Messzeitpunkt einen höheren Rehearsal-Typ erreichten als ein halbes Jahr zuvor und sich ihre Lernleistung ebenfalls verbessert hatte, so wurden sie der Gruppe der „effizienten Strategieentdecker/Aufsteiger“ zugewiesen. Kinder, die den Sprung in eine höhere Rehearsal-Typ-Gruppe ohne Gewinn für die Gedächtnisleistung vollzogen hatten, wurden als „,ineffiziente Strategieentdecker/Aufsteiger“ klassifiziert. Konnte ein Kind sein strategisches Niveau schließlich über ein halbes Jahr konstant halten, so wurde es der Gruppe der „konsistenten Strategen“ zugeordnet.

\subsubsection{Befunde}

Der Blick auf den Prozentsatz von effizienten und ineffizienten Strategieentdeckern in der Gesamtmenge der Aufsteiger/Strategieentdecker (Abbildung 5.9) zeigte, dass bei einigen Kindern die Strategieimplementierung nicht mit einer korrespondierenden Leistungsverbesserung einherging. Damit bestätigt sich die Annahme, dass ein spontaner Strategieeinsatz auch bei der Rehearsal-Strategie nicht immer eine positive Verhaltenskonsequenz im Sinne einer höheren Reproduktionsleistung nach sich zieht. Weiterhin fand sich für den gesamten betrachteten Zeitraum eine weitgehend konstante Anzahl von Aufsteigern/Strategieentdeckern (s.a. Tabelle 5.19-5.21). Jedoch stieg der Anteil an Kindern, für die die spontane Nutzung des strategischen Verhaltens nicht leistungsdienlich ausfiel. 

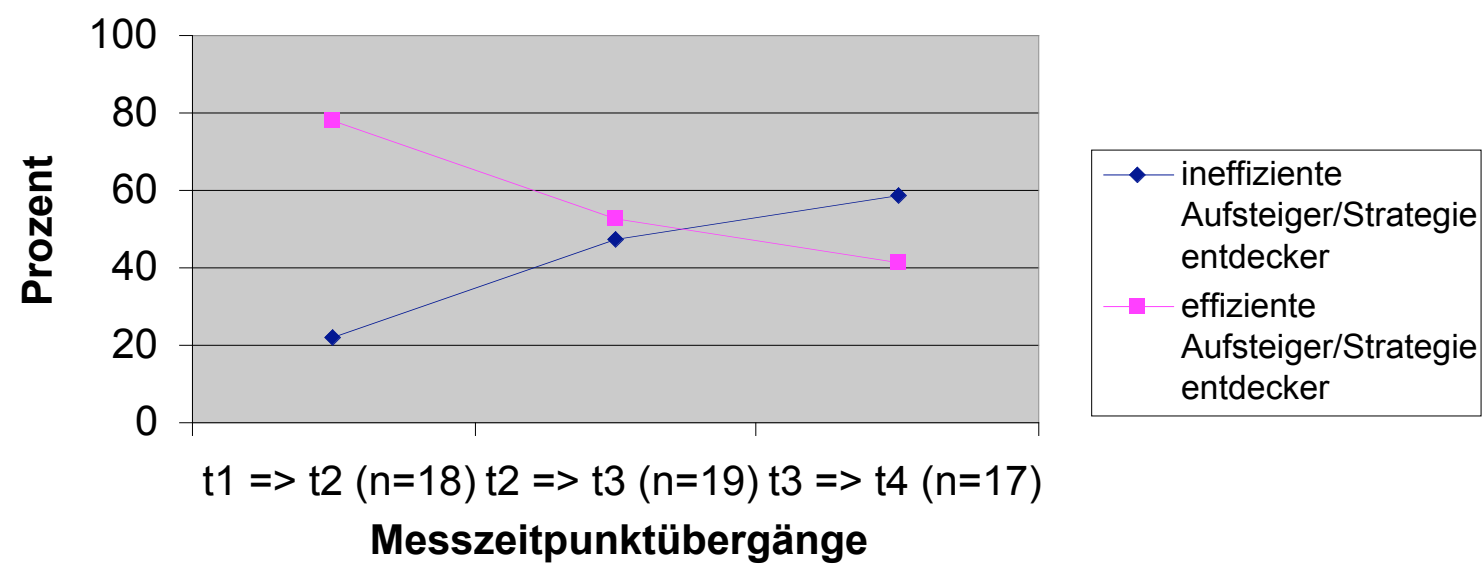

Abbildung 5.9: Prozentsatz von effizienten und ineffizienten Aufsteigern/Strategieentdeckern, getrennt nach Messzeitpunktübergängen.

Vom ersten zum zweiten Messzeitpunkt konnten 18 Kinder ein höheres strategisches Niveau erreichen (Tabelle 5.19). Vier dieser Kinder erzielten keinen adäquaten Nutzen aus ihrer verbesserten strategischen Performanz. Um zu klären, ob es bedeutsame Unterschiede in den Leistungszuwächsen zwischen den Gruppen gab, wurde eine 5 (Gruppe) x 2 (Reproduktionsleistung $\mathrm{t}_{1}, \mathrm{t}_{2}$ ) faktorielle Varianzanalyse mit der Recall-Leistung als messwiederholtem Faktor durchgeführt. Es zeigte sich ein Haupteffekt der Zeit $(F(1,75)=6,81, p<\alpha)$ und eine bedeutsame Zweifachinteraktion zwischen Zeit und Gruppe $(F(4,75)=3.59, p<\alpha)$.

Tabelle 5.19: Mittelwerte und Standardabweichungen der Rehearsal-Set-Größe (R-S-G) und der Reproduktionsleistung (Recall) für die unterschiedlichen Strategengruppen -

Klassifikation auf der Basis des 1. $\left(\mathrm{t}_{1}\right)$ und 2. Messzeitpunktes $\left(\mathrm{t}_{2}\right)$.

\begin{tabular}{|c|c|c|c|c|c|c|c|c|c|c|}
\hline \multirow{7}{*}{} & Nichtstrategen & \multicolumn{2}{c|}{$\begin{array}{c}\text { Absteiger/ } \\
\text { Strat.-verlierer }\end{array}$} & \multicolumn{2}{c|}{$\begin{array}{c}\text { ineffiziente } \\
\text { Aufsteiger/ } \\
\text { Strat.-entdecker } \\
(\mathrm{n}=4)\end{array}$} & \multicolumn{2}{c|}{$\begin{array}{c}\text { effiziente Auf- } \\
\text { steiger/ } \\
\text { Strat.-entdecker } \\
(\mathrm{n}=14)\end{array}$} & \multicolumn{2}{c|}{$\begin{array}{c}\text { konsistente } \\
\text { Strategen } \\
(\mathrm{n}=12)\end{array}$} \\
\cline { 2 - 11 } & $\mathrm{R}-\mathrm{S}-\mathrm{G}$ & Recall & R-S-G & Recall & R-S-G & Recall & R-S-G & Recall & R-S-G & Recall \\
\hline $\mathrm{t}_{1}$ & $\begin{array}{c}.67 \\
(.42)\end{array}$ & $\begin{array}{c}5.27 \\
(1.77)\end{array}$ & $\begin{array}{c}1.88 \\
(.72)\end{array}$ & $\begin{array}{c}4.67 \\
(1.18)\end{array}$ & $\begin{array}{c}.90 \\
(.40)\end{array}$ & $\begin{array}{c}5.5 \\
(.96)\end{array}$ & $\begin{array}{c}.95 \\
(.33)\end{array}$ & $\begin{array}{c}4.62 \\
(1.52)\end{array}$ & $\begin{array}{c}2.69 \\
(.69)\end{array}$ & $\begin{array}{c}5.81 \\
(1.27)\end{array}$ \\
\hline $\mathrm{t}_{2}$ & $\begin{array}{c}.75 \\
(.36)\end{array}$ & $\begin{array}{c}5.31 \\
(1.42)\end{array}$ & $\begin{array}{c}1.31 \\
(.52)\end{array}$ & $\begin{array}{c}6.00 \\
(1.51)\end{array}$ & $\begin{array}{c}2.48 \\
(.46)\end{array}$ & $\begin{array}{c}5.42 \\
(1.03)\end{array}$ & $\begin{array}{c}1.91 \\
(.61)\end{array}$ & $\begin{array}{c}5.90 \\
(1.31)\end{array}$ & $\begin{array}{c}2.81 \\
(.75)\end{array}$ & $\begin{array}{c}5.86 \\
(1.18)\end{array}$ \\
\hline
\end{tabular}

Anschließende Mittelwertsvergleiche ergaben für keinen der beiden Messzeitpunkte Leistungsunterschiede bedeutsamen Ausmaßes zwischen den fünf Gruppen $(t \mathrm{~s} \leq 1.25 /-2.14$, $p s \geq .043$ ). Allerdings zeigten sich signifikante Unterschiede zwischen den Leistungszuwächsen der „effizienten Aufsteiger/Strategieentdecker“ und der Gruppe der „konsistenten Nicht- 
strategen“ $(t(57)=3.16, p<\alpha)$ sowie der Gruppe der „konsistenten Strategen“ $(t(24)=-2.88$, $p<\alpha$ ). Weiterhin gab es eine signifikante Differenz in der Leistungsverbesserung der beiden Aufsteiger-/Strategieentdecker-Gruppen $(t(16)=3.55, p<\alpha)$. Dies kann als deutlicher Hinweis für ein Nutzungsdefizit bei den Kindern, die als ,ineffiziente Strategieentdecker/Aufsteiger“ klassifiziert wurden, angesehen werden.

Tabelle 5.20: Mittelwerte und Standardabweichungen der Rehearsal-Set-Größe (R-S-G) und der Reproduktionsleistung (Recall) für die unterschiedlichen Strategengruppen Klassifikation auf der Basis des 2. $\left(\mathrm{t}_{2}\right)$ und 3. Messzeitpunktes $\left(\mathrm{t}_{3}\right)$.

\begin{tabular}{|c|c|c|c|c|c|c|c|c|c|c|}
\hline \multirow{7}{*}{} & Nichtstrategen & \multicolumn{2}{c|}{$\begin{array}{c}\text { Absteiger/ } \\
\text { Strat.-verlierer } \\
(\mathrm{n}=34)\end{array}$} & \multicolumn{2}{c|}{$\begin{array}{c}\text { ineffiziente } \\
\text { Aufsteiger/ } \\
\text { Strat.-entdecker } \\
(\mathrm{n}=9)\end{array}$} & \multicolumn{2}{c|}{$\begin{array}{c}\text { effiziente Auf- } \\
\text { steiger/ } \\
\text { Strat.-entdecker } \\
(\mathrm{n}=10)\end{array}$} & \multicolumn{2}{c|}{$\begin{array}{c}\text { konsistente } \\
\text { Strategen } \\
(\mathrm{n}=17)\end{array}$} \\
\cline { 2 - 11 } & R-S-G & Recall & R-S-G & Recall & R-S-G & Recall & R-S-G & Recall & R-S-G & Recall \\
\hline $\mathrm{t}_{2}$ & $\begin{array}{c}.72 \\
(.37)\end{array}$ & $\begin{array}{c}5.27 \\
(1.46)\end{array}$ & $\begin{array}{c}1.85 \\
(.62)\end{array}$ & $\begin{array}{c}5.87 \\
(1.33)\end{array}$ & $\begin{array}{c}1.24 \\
(.65)\end{array}$ & $\begin{array}{c}5.74 \\
(1.57)\end{array}$ & $\begin{array}{c}1.06 \\
(.48)\end{array}$ & $\begin{array}{c}5.43 \\
(1.13)\end{array}$ & $\begin{array}{c}2.70 \\
(.72)\end{array}$ & $\begin{array}{c}5.86 \\
(1.21)\end{array}$ \\
\hline $\mathrm{t}_{3}$ & $\begin{array}{c}.69 \\
(.44)\end{array}$ & $\begin{array}{c}5.58 \\
(1.38)\end{array}$ & $\begin{array}{c}1.05 \\
(.66)\end{array}$ & $\begin{array}{c}5.83 \\
(2.25)\end{array}$ & $\begin{array}{c}2.71 \\
(.76)\end{array}$ & $\begin{array}{c}5.41 \\
(1.44)\end{array}$ & $\begin{array}{c}2.17 \\
(.84)\end{array}$ & $\begin{array}{c}6.60 \\
(1.24)\end{array}$ & $\begin{array}{c}3.12 \\
(.89)\end{array}$ & $\begin{array}{c}6.22 \\
(1.24)\end{array}$ \\
\hline
\end{tabular}

Auch für den Vergleich von Messzeitpunkt 2 und 3 (Tabelle 5.20) zeigten sich der Haupteffekt der Zeit $(F(1,75)=2.66, p<\alpha)$ und die Zweifachinteraktion von Zeit und Gruppenzuordnung $(F(4,75)=2.70, p<\alpha)$. Weiterhin bestanden zu keinem der beiden Messzeitpunkte signifikante Leistungsdifferenzen zwischen den Gruppen ( $t \mathrm{~s} \leq .78 /-2.1, p \mathrm{~s} \geq .043)$. Wie bereits zuvor konnten auch beim Übergang vom zweiten zum dritten Erhebungszeitpunkt Unterschiede im Leistungszuwachs der Kinder in der Gruppe der „effizienten Aufsteiger/Strategieentdecker“ verglichen mit a) der Gruppe der „Absteiger/Strategieverlierer“ $(t(18)=2.23$, $p<\alpha)$, b) den ,ineffizienten Aufsteigern/Strategieentdeckern“ $(t(17)=4.50, p<\alpha)$ und c) der Gruppe der „,konsistenten Strategen“ $(t(25)=-2.29, p<\alpha)$ verzeichnet werden.

Abschließend erbrachte auch die Analyse des Übergangs vom dritten zum vierten Messzeitpunkt (Tabelle 5.21) den Haupteffekt der Zeit $(F(1,75)=7,14, p<\alpha)$ und den Interaktionseffekt von Zeit und Gruppenzugehörigkeit $(F(4,75)=6.46, p<\alpha)$. Beim vierten Erhebungszeitpunkt fanden sich darüber hinaus tendenzielle Differenzen in der Lernleistung von „Nichtstrategen“ und „effizienten Aufsteigern/Strategieentdeckern“ $(t(21.25)=-2.63, p<\alpha)$, „konsistenten Strategen“ und „konsistenten Nichtstrategen“ $(t(53)=-2.57, p<\alpha)$ sowie ,ineffizienten“ und „effizienten Aufsteigern“ $(t(15)=-3.22 p<\alpha)$. Da das festgelegte Signifikanzniveau nach der Korrektur durch die Alpha-Adjustierung nach Bonferroni bei $p=\alpha / 10=.005$ lag, 
konnten diese Differenzen jedoch nicht als signifikant eingestuft werden. Das gleiche galt für einen Leistungsunterschied zwischen den „effizienten Aufsteigern/Strategieentdeckern“ und den „,konsistenten Strategen“ beim dritten Messzeitpunkt ( $t(30)=-2.21, p>\alpha / 10)$. Allerdings erwies sich die Differenz in der Recall-Leistung von „ineffizienten Strategen“ und „konsistenten Strategen“ bei Messzeitpunkt 4 mit $t(33)=-3.45(p=.002)$ als signifikant.

Tabelle 5.21: Mittelwerte und Standardabweichungen der Rehearsal-Set-Größe (R-S-G) und der Reproduktionsleistung (Recall) für die unterschiedlichen Strategengruppen -

Klassifikation auf der Basis des 3. $\left(\mathrm{t}_{3}\right)$ und 4. Messzeitpunktes $\left(\mathrm{t}_{4}\right)$.

\begin{tabular}{|c|c|c|c|c|c|c|c|c|c|c|}
\hline \multirow{7}{*}{} & Nichtstrategen & \multicolumn{2}{c|}{$\begin{array}{c}\text { Absteiger/ } \\
\text { Strat.-verlierer } \\
(\mathrm{n}=30)\end{array}$} & \multicolumn{2}{c|}{$\begin{array}{c}\text { ineffiziente } \\
\text { Aufsteiger/ } \\
\text { Strat.-entdecker } \\
(\mathrm{n}=10)\end{array}$} & $\begin{array}{c}\text { effiziente Auf- } \\
\text { steiger/ } \\
\text { Strat.-entdecker } \\
(\mathrm{n}=7)\end{array}$ & \multicolumn{2}{c|}{$\begin{array}{c}\text { konsistente } \\
\text { Strategen } \\
(\mathrm{n}=25)\end{array}$} \\
\cline { 2 - 11 } & $\mathrm{R}-\mathrm{S}-\mathrm{G}$ & Recall & $\mathrm{R}-\mathrm{S}-\mathrm{G}$ & Recall & R-S-G & Recall & R-S-G & Recall & R-S-G & Recall \\
\hline $\mathrm{t}_{3}$ & $\begin{array}{c}.67 \\
(.43)\end{array}$ & $\begin{array}{c}5.61 \\
(1.69)\end{array}$ & $\begin{array}{c}2.37 \\
(.99)\end{array}$ & $\begin{array}{c}6.29 \\
(1.79)\end{array}$ & $\begin{array}{c}1.12 \\
(.48)\end{array}$ & $\begin{array}{c}6.17 \\
(1.39)\end{array}$ & $\begin{array}{c}.98 \\
(.71)\end{array}$ & $\begin{array}{c}5.00 \\
(1.04)\end{array}$ & $\begin{array}{c}3.04 \\
(.77)\end{array}$ & $\begin{array}{c}6.12 \\
(1.22)\end{array}$ \\
\hline $\mathrm{t}_{4}$ & $\begin{array}{c}.79 \\
(.40)\end{array}$ & $\begin{array}{c}6.00 \\
(1.51)\end{array}$ & $\begin{array}{c}1.32 \\
(.57)\end{array}$ & $\begin{array}{c}5.88 \\
(1.75)\end{array}$ & $\begin{array}{c}2.66 \\
(1.28)\end{array}$ & $\begin{array}{c}5.53 \\
(1.05)\end{array}$ & $\begin{array}{c}2.54 \\
(1.07)\end{array}$ & $\begin{array}{c}7.00 \\
(.69)\end{array}$ & $\begin{array}{c}3.31 \\
(.95)\end{array}$ & $\begin{array}{c}6.93 \\
(1.10)\end{array}$ \\
\hline
\end{tabular}

Deutlich kleinere Leistungszuwächse konnten für die Gruppe der ,ineffizienten Aufsteiger/Strategieentdecker“ im Vergleich zu a) den „konsistenten Nichtstrategen“, b) den „effizienten Aufsteigern/Strategieentdeckern“ und c) den „konsistenten Strategen“ verzeichnet werden $(t \mathrm{~s} \geq 2.14, p s<\alpha)$. Für die Gruppe der ,effizienten Aufsteiger/Strategieentdecker“" ergaben sich weiterhin bedeutsam größere Steigerungen in der Lernleistung im Vergleich zu a) den ,konsistenten Nichtstrategen“, b) den „Absteigern/Strategieverlierern“ und c) den „konsistenten Strategen $\left(t \mathrm{~s} \geq 2.62, p_{\mathrm{s}}<\alpha\right)$. Mit $t(31)=2.75(p<\alpha)$ konnte letztlich auch ein deutlicherer Leistungszuwachs der „,konsistenten Strategen“ gegenüber der Gruppe der „Absteiger/Strategieverlierer" nachgewiesen werden. Das Muster statistisch bedeutsamer Unterschiede von Leistungszuwächsen zwischen Gruppen wird in Tabelle 5.22 zusammengefasst.

Besonders die berichteten Unterschiede im Leistungszuwachs zwischen „effizienten Aufsteigern/Strategieentdeckern“ und „ineffizienten Aufsteigern/Strategieentdeckern“ bei aufeinanderfolgenden Datenerhebungen und die fehlende Differenz zwischen „Nichtstrategen“ und ,ineffizienten Aufsteigern/Strategieentdeckern“ können als Evidenz für eine Nutzungsineffizienz bei der Anwendung von kumulativem Rehearsal gewertet werden. 
Tabelle 5.22: Auflistung von Gruppen mit signifikanten Differenzen in den Leistungszuwächsen zwischen zwei Messzeitpunkten.

\begin{tabular}{|c|c|}
\hline $\begin{array}{l}\text { Messzeitpunkt- } \\
\text { übergänge }\end{array}$ & Gruppen mit bedeutsamen Differenzen in den Leistungszuwächsen \\
\hline$t_{1}=>t_{2}$ & $\begin{array}{l}\text { „effiziente Aufsteiger/Strategieentdecker“ > „Nichtstrategen“ } \\
\text { „effiziente Aufsteiger/Strategieentdecker“ > „Strategen“" } \\
\text { „,effiziente Aufsteiger/Strategieentdecker“ > } \\
\text { ineffiziente Aufsteiger/Strategieentdecker“ }\end{array}$ \\
\hline$t_{2}=>t_{3}$ & $\begin{array}{l}\text { „,effiziente Aufsteiger/Strategieentdecker“ }>\text { „Absteiger/Stategieverlierer“ } \\
\text { „effiziente Aufsteiger/Strategieentdecker“ > „konsistente Strategen“" } \\
\text { „effiziente Aufsteiger/Strategieentdecker“ }> \\
\text { „ineffiziente Aufsteiger/Strategieentdecker“ }\end{array}$ \\
\hline $\mathrm{t}_{3}=>\mathrm{t}_{4}$ & $\begin{array}{l}\text { „effiziente Aufsteiger/Strategieentdecker“ > „Nichtstrategen“ } \\
\text { „effiziente Aufsteiger/Strategieentdecker“ > „Strategen“ } \\
\text { „effiziente Aufsteiger/Strategieentdecker“ }> \\
\text { „,ineffiziente Aufsteiger/Strategieentdecker“ } \\
\text { „effiziente Aufsteiger/Strategieentdecker“ > „Absteiger/Stategieverlierer“ } \\
\text { „Nichtstrategen“ > „ineffiziente Aufsteiger/Strategieentdecker“ } \\
\text { „konsistente Strategen“ > ,ineffiziente Aufsteiger/Strategieentdecker“ } \\
\text { „konsistente Strategen“ > „Absteiger/Strategieverlierer“ }\end{array}$ \\
\hline
\end{tabular}

Bei der Suche nach möglichen Einflussfaktoren bzw. Prädiktoren der effizienten vs. ineffizienten Strategieimplementierung werden in der Literatur sowohl kapazitive (z.B. Coyle \& Bjorklund, 1997) als auch metakognitive Aspekte (z.B. Hasselhorn, 1996) und Mängel in der Wissensbasis (z.B. Bjorklund \& Douglas, 1997) diskutiert. Theoretisch könnten auch verschiedene Kombinationen dieser Faktoren oder unterschiedliche Mechanismen zu unterschiedlichen Zeitpunkten für das Auftreten einer Nutzungsineffizienz verantwortlich sein. Für die vorliegenden Daten zeigten vertiefende Analysen, dass zu keinem Zeitpunkt bedeutsame Differenzen in den beiden Maßen der Gedächtniskapazität zwischen den beiden Gruppen von Aufsteigern/Strategieentdeckern existierten $(t \mathrm{~s} \leq 2.08, p \mathrm{~s} \geq .055)$. Ebenso wenig ließ sich ein unterschiedliches Ausmaß an strategiespezifischem Metagedächtnis bei Messzeitpunkt 3 oder 4 finden $(t s \leq 1.14, p s \geq .27)$. Die Berechnung von Unterschieden in den korrelativen Zusammenhängen von Rehearsal-Set-Größen und Reproduktionsleistungen zwischen den beiden Gruppen erbrachte ebenfalls keinen signifikanten Befund ( $t \mathrm{~s} \leq .86 /-.20, p s \geq .39)$. Somit können sowohl kapazitive und metakognitive Faktoren als auch Aspekte der unterschiedlichen Emer- 
genz strategischer Prozesse als Ursache für die gefundenen Unterschiede zwischen den Gruppen der effizienten und ineffizienten Aufsteiger/Strategieentdecker ausgeschlossen werden.

Die Frage nach einer Nutzungsineffizienz kumulativen Rehearsals kann auf der Grundlage der Befunde positiv beantwortet werden. Bei jedem Messzeitpunkt wurde eine Gruppe mit Kindern identifiziert, die durch die erste spontane Anwendung kumulativer Memorierprozesse bzw. deren erstmaligem Gebrauch als dominante Gedächtnisstrategie keinen Leistungsgewinn erzielten. Weder kapazitive noch metakognitive Aspekte konnten als Ursache für diese mangelnde Emergenz der strategischen Prozesse dieser Kinder identifiziert werden.

\section{Diskussion}

\subsection{Zusammenfassung der empirischen Befunde}

Auf der Basis eines kombinierten Querschnitt-Längsschnitt-Designs wurden zentrale Annahmen zur Entwicklung von Rehearsal-Prozessen und ihrem Zusammenhang zur Reproduktionsleistung überprüft. Dabei wurde zwischen drei Betrachtungsebenen unterschieden. Auf der ersten Ebene erfolgte der klassisch-konventionelle Vergleich dreier Altersgruppen hinsichtlich der Unterschiede in der Gedächtnisleistung, im Memorierverhalten und im Zusammenhang zwischen diesen Faktoren. Auf der zweiten Ebene wurden längsschnittliche Analysen durchgeführt, um die angesprochenen Veränderungen sowie deren Beziehungen und zeitliche Stabilitäten im Verlauf der späten Grundschuljahre genauer zu beleuchten. Die Erfassung von intraindividuellen Entwicklungsverläufen und der interindividuellen Differenzen in den intraindividuellen Veränderungen waren Gegenstand der dritten Betrachtungsebene. Ergänzend fanden hier vertiefende Überlegungen zu Abrufphänomenen statt. Die Entwicklungsanalysen wurden durch die Überprüfung von metakognitiven und kapazitiven Faktoren als zentralen Antezedenzien der verbesserten strategischen Performanz und der Antwort auf die Frage nach der Leistungsdienlichkeit erster spontaner Strategieanwendungen vervollständigt. Im Folgenden sollen die wichtigsten Ergebnisse der Studie zusammengefasst werden.

Querschnittlicher Altersvergleich: Analog zu früheren Befunden der Arbeitsgruppe um Ornstein konnten bedeutsame Unterschiede in der Reproduktionsleistung und der mittleren Rehearsal-Set-Größe zwischen Zweit-, Viert- und Sechstklässlern verzeichnet werden. Überraschenderweise zeigte sich keine alterskorrelierte Zunahme im Zusammenhang zwischen diesen beiden Faktoren. Auf keiner der drei Altersstufen war ein systematischer Zusammenhang zwischen der strategischen Variable und der Gedächtnisleistung erkennbar. 
Längsschnittliche Entwicklungsanalysen auf Gruppenebene: Auch bei der Betrachtung der Veränderungen von der zweiten bis zur vierten Klassenstufe konnte eine signifikante und kontinuierliche Zunahme der durchschnittlichen Reproduktionsleistung und der mittleren Rehearsal-Set-Größe registriert werden. Beide Faktoren wiesen eine deutliche zeitliche Stabilität zwischen zwei aufeinanderfolgenden Erhebungen und über die gesamte Zeitspanne auf. Die Korrelationen lagen jedoch unter den bislang in der Literatur berichteten Werten. Der korrelative Zusammenhang zwischen der Gedächtnis- und der Strategievariablen stieg vom Ende der zweiten zum Anfang der dritten Klasse an, fiel ein halbes Jahr später unter das Signifikanzniveau und erreichte zum Anfang der vierten Klasse wieder einen statistisch signifikanten Wert.

Ebene der intraindividuellen Entwicklungsverläufe: Die Erfassung der genutzten Gedächtnisstrategien ergab einen Anstieg in der Anzahl von Kindern, die mehr als eine Strategie verwendet hatten. Ebenso stieg zwischen Ende der zweiten und Anfang der vierten Klasse der Anteil an Kindern, die eine Strategie primär, also im Sinne einer Hauptstrategie, einsetzten. Auf der Basis einer Klassifikation strategischer Performanz konnte gezeigt werden, dass sich die Entdeckung und der verstärkte Gebrauch von kumulativem Rehearsal wesentlich zwischen zweiter und dritter Klasse sowie im Verlauf des dritten Schuljahres vollzog. Allerdings war damit noch nicht zwingenderweise ein Leistungsvorteil verbunden. Dieser stellte sich erst zu Beginn der vierten Klassenstufe ein. Im Gegensatz zu früheren Arbeiten (z.B. Guttentag et al., 1987) erwies sich der Anteil von Kindern, die ein single-item-rehearsal verwendeten, als sehr gering. Die Analyse der Verlaufsdaten ergab Belege für eine gewisse Stabilität der vorgenommenen Einteilung und einen generellen Trend $\mathrm{zu}$ einem elaborierteren strategischen Verhalten im Verlauf der späten Grundschuljahre. Es fanden sich keine Hinweise für einen abrupten Prozess von Strategieaneignung und -verlust (Achterbahn-Phänomen). Wie bereits in anderen Studien konnte eine Überlegenheit der Kinder mit kumulativer Memoriertätigkeit im Abruf früher und mittlerer Listenitems verzeichnet werden (prerecency sections). Überraschenderweise zeigte sich weiterhin ein deutlicher Vorteil der Kinder, die kein oder kaum kumulatives Rehearsal eingesetzt hatten bei der Erinnerung der Items vom Ende der Lernliste.

Die Reproduktionskurven aller Rehearsal-Typ-Gruppen wiesen mehrfach U-förmige Verläufe auf. Diese für Listen mit unverbundenen Items ungewöhnliche Verlaufsstruktur wurde als Indiz für Organisationsprozesse in der Abrufphase gewertet, die durch die Memoriertätigkeit initiiert worden waren. Kinder, die kumulatives Rehearsal praktiziert hatten, nutzten zum Ende der dritten und bei Beginn der vierten Klasse vermehrt die serielle Präsentationsreihenfolge als Abrufhilfe. Am Ende der zweiten und zu Beginn der dritten Klasse tra- 
ten keine signifikanten Differenzen zwischen den Rehearser-Gruppen auf. Im Verlauf der dritten Klasse konnten Hinweise auf Unterschiede in den Inter-Response-Zeiten zwischen den Kindern der „Dominant-cumulative“-Gruppe und den restlichen Kindern entdeckt werden. Weiterhin zeigte sich für kumulative Rehearser das Phänomen des response bursting mit signifikant niedrigeren Interitemlatenzen zwischen Wörtern aus einem (kumulativen) RehearsalSet im Vergleich zu Itemlatenzen zwischen Wörtern aus unterschiedlichen Rehearsal-Sets.

Die Bedeutung metakognitiver und kapazitiver Aspekte - Überprüfung der Vorhersagen der Strategie-Emergenz-Theorie: Das beim dritten und vierten Erhebungszeitpunkt erfasste strategiespezifische Metagedächtnis für Rehearsal nahm vom Ende der dritten zum Anfang der vierten Klasse bedeutsam zu. Zu keinem der beiden Zeitpunkte konnte jedoch ein relevanter Zusammenhang zwischen dem strategiespezifischen Metagedächtnis und der Rehearsal-Set-Größe nachgewiesen werden. Allerdings verzeichneten die Rehearsal-Strategen deutlichere Zuwächse in ihrem Ausmaß an Metakognition und ihrer Recall-Leistung als die Nichtstrategen. Auch insgesamt zeigte sich bei einem strengen Strategenkriterium (vgl. Hasselhorn \& Lindner-Müller, 1995) ein Erinnerungsvorteil der Rehearsal-Strategen gegenüber den Nichtstrategen beim dritten und vierten Messzeitpunkt.

Sowohl für die Zahlenspanne vorwärts als auch für die Zahlenspanne rückwärts konnte ein allgemeiner Anstieg von Ende der zweiten bis Anfang der vierten Klasse festgestellt werden. Differenziertere Analysen erbrachten Belege für einen bedeutsamen Einfluss kapazitiver Aspekte beim Erwerb kumulativer Memorierprozesse. Im späteren Verlauf nahm bei kumulativen Rehearsern die Bedeutung von Exekutivfunktionen für die Rehearsal-Qualität zu, also die Fähigkeit, zwischen Suchräumen hin und herzuschalten und Informationen aus der Wissensbasis abzurufen bzw. zu manipulieren. In diesem Stadium profitierten RehearsalStrategen auch stärker von ihrer guten Gedächtniskapazität, da die mentalen Anforderungen der kumulativen Lernaktivität durch Automatisierungsprozesse reduziert worden waren.

Belege für eine Nutzungsineffizienz bei kumulativem Rehearsal: Erstmals wurde in der vorliegenden Studie versucht, empirische Belege für eine Nutzungsineffizienz strategischer Rehearsal-Tätigkeit zu finden, d.h. für das Ausbleiben eines Lerngewinns bei der ersten spontanen Anwendung kumulativer Memorierprozesse bzw. deren erstmaliger Gebrauch als dominante Gedächtnisstrategie. Bei allen Übergängen zwischen zwei Erhebungszeitpunkten konnte eine Subgruppe mit Kindern identifiziert werden, die als nutzungsineffizient einzustufen waren. Die Größe dieser Gruppe war im Vergleich zu der Anzahl von Kindern, die aus ihrem fortgeschritten Lernverhalten Gewinn zogen, anfangs klein (4 von 18 Kindern). Im Verlauf der dritten und beim Übergang zur vierten Klasse war das Verhältnis von „effizien- 
ten“ und „,ineffizienten Aufsteigern/Strategieentdeckern“ etwa gleich. Weder die Betrachtung von kapazitiven noch von metakognitiven Aspekten als mögliche Ursache für die mangelnde Effizienz strategischer Prozesse bei den als nutzungsineffizient klassifizierten Kindern erbrachte überzeugende Belege für die relevanten Differenzen zwischen den beiden Gruppen von Aufsteigern/Strategieentdeckern.

\subsection{Beantwortung der zentralen Fragestellungen}

Die Antworten auf die in Abschnitt 3 formulierten zentralen Fragen und Hypothesen, die der vorliegenden Untersuchung zu Grunde liegen, erfolgen getrennt für die eingenommenen Betrachtungsebenen.

Für den klassisch-konventionellen Vergleich der drei Altersgruppen, der die Basis für die meisten bisherigen Untersuchungen zu Rehearsal-Prozessen darstellt, konnten die gewohnten Differenzen in der durchschnittlichen Reproduktionsleistung und der Rehearsal-SetGröße zwischen den drei Klassenstufen verzeichnet werden. Umso erstaunlicher war das Fehlen eines bedeutungsvollen Zusammenhangs zwischen diesen beiden Variablen für die Viert- und Sechstklässler. Dieser Befund alleine ließe an der Bedeutung von Memorierprozessen für den alterskorrelierten Anstieg der Gedächtnisleistung zweifeln. Das NichtEintreten der entsprechenden psychologischen Vorhersage würde somit zu einer negativen Entscheidung hinsichtlich der psychologischen Hypothese auf der ersten Betrachtungsebene führen.

Die Überprüfung der Streudiagramme für die beiden höheren Klassenstufen signalisierte jedoch die Existenz von zwei Fraktionen innerhalb der Altersgruppen, für die unterschiedliche Zusammenhänge verzeichnet werden konnten. Sowohl bei den Viert- als auch bei den Sechstklässlern gab es eine Gruppe mit Kindern, die trotz kleiner durchschnittlicher Rehearsal-Sets $(<2.0)$ gute bis sehr gute Reproduktionsleistungen erbrachten. Der Zusammenhang zwischen der Strategie- und der Leistungsvariable fiel dabei für die $n=29$ nichtstrategischen Viertklässler mit $r=-.05(p=.80)$ sehr klein aus, während für die $\mathrm{n}=12$ nichtstrategischen Sechstklässler mit $r=-.67(p<\alpha)$ sogar eine signifikant negative Korrelation verzeichnet werden konnte.

Es scheint sich hier vor allem bei der sechsten Klassenstufe um Kinder zu handeln, die entweder über eine sehr gute Arbeitsgedächtniskapazität verfügen und daher verbale Gedächtnisstrategien gewissermaßen ,nicht nötig“ haben, oder aber um Kinder, die entgegen der Instruktion nur verdeckt memorierten. Für die restlichen Kinder beider Altersgruppen konnte 
ein deutlicher Zusammenhang zwischen der Qualität des Rehearsals und der Lernleistung festgestellt werden. Dieser fiel für die $n=11$ Viertklässler mit einem durchschnittlichen Rehearsal-Set $\geq 2.0$ noch nicht signifikant aus $(r=.11 ; p=.75)$. Für die zwei Jahre älteren Kinder konnte mit $r=.40(p<\alpha)$ bei einer Gruppengröße von $\mathrm{n}=28$ jedoch ein deutlicher Einfluss der Strategie auf die Lernleistung verzeichnet werden. Der fehlende korrelative Zusammenhang zwischen der Rehearsal-Set-Größe und Lernleistung bei den Viertklässlern kann zwar durch die geringe Teststärke (Power) aufgrund des kleinen Stichprobenumfangs verursacht worden sein, weitaus plausibler und übereinstimmend mit früheren Befunden (z.B. Hasselhorn \& Lindner-Müller, 1995) ist jedoch die Annahme einer (noch) geringen Leistungsdienlichkeit des strategischen Verhaltens auf dieser Altersstufe.

Bei der längsschnittlichen Betrachtung auf Gruppenebene fällt die Antwort für die Annahme einer alterskorrelierten Zunahme der Qualität des Memorierverhaltens und der Gedächtnisleistung sowie eines mit dem Alter steigenden Zusammenhangs zwischen diesen Faktoren positiv aus. Auf dieser zweiten Ebene fanden sich kontinuierliche und signifikante Zuwächse in der durchschnittlichen Rehearsal-Set-Größe und der Reproduktionsleistung sowie ein Anstieg des Zusammenhangs zwischen den beiden Faktoren über die Gesamtzeit. Das vorliegende Befundmuster deutet jedoch an, dass es in dem betrachteten Zeitraum keinen linearen Anstieg im Einfluss des Rehearsals auf die Gedächtnisleistung gibt. Der Rückgang der Korrelation analog mit einer Verminderung der zeitlichen Stabilität der Strategievariablen spricht für das Auftreten von qualitativen Veränderungen im Memorierverhalten, die jedoch nicht sofort eine Leistungssteigerung mit sich bringen.

Ähnlich wie in der Studie von Kunzinger (1985) konnte ein alterskorrelierter Anstieg der Variabilität in der Strategievariable nachgewiesen werden. Dies kann als Hinweis auf eine Zunahme der interindividuellen Differenzen in den intraindividuellen Veränderungen angesehen werden. Während die Probanden in der Studie von Kunzinger (1985) jedoch bereits in einem Alter von 7;5 Jahren eine durchschnittliche Set-Größe von $M=1.7(S D=0.9)$ aufwiesen, die sich zwei Jahre später auf $M=2.6(S D=1.3)$ erhöht hatte, lag der Ausgangswert in der vorliegenden Untersuchung für etwas ältere Kindern ( $7 ; 11$ Jahre) bei $M=1.11(S D=0.86)$. Der von Kunzinger (1985) berichtete Ausgangswert wurde erst im Alter von 9;1 Jahren erreicht (vgl. Abschnitt 5.1.2, Tabelle 5.1). Das höhere Niveau strategischer Performanz, auf dem sich die Kinder in der Studie von Kunzinger (1985) befinden, lässt sich durch die indirekte Aufforderung zur Nutzung kumulativen Rehearsals erklären. Dabei wurden die Kinder, die beim Lernen einer Übungsliste singuläres Rehearsal anwendeten „,...informed of the alternative strategy of rehearsing more than one item at a time ..." (Kunzinger, 1985, S. 643). 
Die Überprüfung der zeitlichen Stabilitäten der Reproduktionsleistung und der Rehearsal-Set-Größe zeigte für beide Variablen signifikante Interkorrelationen zwischen den Erhebungszeitpunkten und bestätigte damit die entsprechende psychologische Hypothese. Die gefundenen Werte lagen zwar unter den in der Literatur berichteten Angaben (vgl. Kunzinger, 1985), sprechen jedoch deutlich für eine gewisse Kontinuität in der Entwicklung des Memorierverhaltens zwischen 8 und 10 Jahren und gegen einen sprunghaften Wechsel von Strategieerwerb und -verlust (Achterbahn-Phänomen).

Die vorliegenden Daten stützen jedoch nicht die an anderer Stelle formulierte Annahme, dass alle Kinder sich in diesem Altersspektrum in einer etwa gleichen Geschwindigkeit entwickeln bzw. die gleichen Entwicklungsstufen durchlaufen (vgl. Schneider \& Sodian, 1997). Aus der Kombination von mittleren Korrelationskoeffizienten und einem alterskorrelierten Anstieg der Standardabweichung in der Strategievariable kann auf dieser Betrachtungsebene eher von einer allgemeinen Tendenz zu qualitativen Veränderungen in Form von kumulativem Rehearsal und gleichzeitig von einer wachsenden Variabilität in den Entwicklungsverläufen ausgegangen werden.

Die Analyse der intraindividuellen Entwicklungsverläufe lieferte an verschiedenen Stellen ein von bisherigen Befunden und den auf der Grundlage von Altersvergleichen gebildeten impliziten Annahmen abweichendes Bild. Die deskriptive Betrachtung der genutzten Strategien zeigte z.B. einen erstaunlich hohen Anteil an Labeling und einen ungewöhnlich geringen Anteil an singulärem Rehearsal. Besonders der letzte Aspekt widerspricht früheren Befunden, nach denen Grundschulkinder beim Lernen einer supraspan word list spontan einen singulären Memorieransatz wählen (Guttentag et al., 1987). Der Anteil an kumulativem Rehearsal und die durchschnittliche Rehearsal-Set-Größe beim ersten Messzeitpunkt entsprachen dagegen den in anderen Studien berichteten Werten für Zweitklässler. Ebenso stimmte die durchschnittliche Rehearsal-Set-Größe beim zweiten Messzeitpunkt exakt mit dem Wert der Gleichaltrigen in der Studie von Guttentag et al. (1987) überein. Ein Jahr später lag der Wert mit $M=2.00(S D=1.40)$ deutlich unterhalb dem der gleichaltrigen Viertklässler in der Studie von Guttentag et al. (1987) und über dem der untersuchten Querschnitt-Kontrollstichprobe. Die Reproduktionsleistungen waren mit denen der Kinder bei Guttentag et al. (1987) vergleichbar.

Über den erfassten Zeitraum konnte ein Anstieg an Kindern verzeichnet werden, die einen multiplen Strategieeinsatz mit zwei oder mehr Mnemotechniken zeigten. Dies deckt sich mit der Annahme Sieglers (1996), dass Kinder zu jedem Zeitpunkt und für jede Aufgabe eine Vielfalt von Strategien verfügbar haben, unabhängig davon, ob sie diese auch nutzten. Paral- 
lel dazu fand sich eine mit dem Alter zunehmende Tendenz zum Gebrauch einer Hauptstrategie, d.h. die Nutzung einer Mnemotechnik in mehr als 50 Prozent der Lernmöglichkeiten. Ein damit einhergehender Leistungsvorteil einer Gruppe konnte erst beim vierten Messzeitpunkt festgestellt werden.

Die Annahmen über einen deutlichen Anstieg in der Anzahl der als „Dominantcumulative“-Rehearser klassifizierten Kinder - speziell in der zweiten Hälfte des erfassten Zeitraums - wurde durch die Befunde bestätigt. Eine im Vergleich zu den anderen Gruppen bessere Gedächtnisleistung konnte jedoch wiederum erst beim vierten Erhebungszeitpunkt verzeichnet werden. Aufgrund der kleinen Gruppengrößen war ein bedeutsamer Unterschied jedoch auch nicht früher zu erwarten. Berücksichtigt man noch die Anzahl von Kindern, die das kumulative Rehearsal in geringerem Maße bzw. nicht so konsistent eingesetzt hatten, so gebrauchten am Anfang der vierten Klasse 65 Prozent der Kinder die „Königsstrategie“ bei der Bearbeitung der seriellen Lernaufgabe.

Die Überprüfung der zeitlichen Stabilitäten und der Verlaufsmuster in der vorliegenden Untersuchung sprechen auch auf der dritten Betrachtungsebene für eine weitgehend kontinuierlich-progressive Entwicklung der Memorierstrategie im Verlauf der späten Grundschuljahre - ohne dass allgemeingültige Stufen oder Verlaufstypen identifiziert werden konnten. Dieser Annahme widerspricht auch nicht das Vorliegen von drei Entwicklungstypen im Kategorienmuster der Konfigurationsfrequenzanalyse, da es sich bei zwei dieser Typen um Plateaus ohne Entwicklungsmoment handelt (31 Prozent konsistent „Non-cumulative“- und 11 Prozent konsistent „Dominant-cumulative“-Rehearser). Die gefundenen statistisch bedeutsamen Entwicklungsschübe stützen die Vermutung, dass es in der zweiten Hälfte der Grundschulzeit zu einer Entdeckung und anschließend verstärkten Nutzung von kumulativem Rehearsal kommt. Die große Anzahl individueller Verlaufsmuster, die dabei gefunden wurde, verdeutlichen die große Variabilität in der Entwicklung und sprechen gegen klare Phasen oder Stufen, die von allen Kindern durchlaufen werden.

Obwohl die Betrachtung der Entwicklungsverläufe die Annahme zulässt, dass es auch zu einem Verlust der kumulativen Memorierstrategie kommen kann, sprechen die empirischen Befunde insgesamt stärker für den Erhalt und die Erweiterung einer einmal erworbenen mnemotechnischen Kompetenz. Ein wie in der LOGIK-Studie für die Organisationsstrategie gefundener Wechsel von Strategieerwerb-Strategieverlust-Strategiewiedererwerb für einen großen Prozentsatz der untersuchten Kinder (vgl. Sodian \& Schneider, 1999) konnte in der vorliegenden Studie für die Memorierstrategie von Einzelfällen abgesehen nicht beobachtet werden. Allerdings vollzog sich bei einigen Kindern die Akquisition kumulativen Rehearsals 
als ein Alles-oder-Nichts-Sprung von einem „Non-cumulative“-Status zu einem „Dominantcumulative“-Status. Dies kann zum einen dadurch erklärt werden, dass sich ein möglicher gradueller Anstieg der strategischen Performanz in dem halben Jahr zwischen den Erhebungszeitpunkten abspielt, zum anderen erlaubt die an dieser Stelle vorgenommene Klassifikation keine Differenzierung zwischen Labeling und singulärem Rehearsal als Vorstufen strategischer Kompetenz.

Der gefundene primacy-recency trade-off mit signifikant besseren Reproduktionsleistungen der „Dominant-cumulative“-Rehearser im Primacy Bereich der seriellen Positionskurve und einem Erinnerungsvorteil der „Non-cumulative“-Rehearser bei den Enditems widerspricht dem gängigen Befundmuster. Frühere Arbeiten zeigten zwar einen Leistungsvorteil im Bereich der frühen und mittleren Listenitems (prerecency sections) durch kumulatives Rehearsal, aber „recall at the recency posistions was identical for subjects who rehearsed differently“ (Ornstein, Naus \& Stone, 1977, S. 23). Ein mit dem vorliegenden Befundmuster vergleichbarer Verlauf bei second-grade many-item subjects und sixth-grade spontaneous and many-item subjects in der Studie von Ornstein, Naus und Stone (1977) führte bei den Autoren zu der Vermutung, dass es sich um einen Modalitätseffekt handelt. Danach kommt es bei akustischer Präsentation der Lernitems zu einer Interferenz zwischen aktiv (d.h. kumulativ) memorierten Items und aktuell präsentierten Items. Dieser Interpretation zufolge sollte bei einer audiovisuellen Präsentation, wie sie in der vorliegenden Studie realisiert wurde, kein Informationsverlust später Listenitems durch Interferenzeffekte auftreten, da zusätzlich zur verbalen eine visuelle Repräsentation eines Items existiert (vgl. Ornstein, Naus \& Stone, 1977).

Eine solche Annahme lässt sich durch die Daten nicht bestätigen. Aus der Analyse des Lernverhaltens der „Dominant-cumulative“-Rehearser kann auch nicht der Schluss gezogen werden, dass die Kinder dieser Gruppe sich mehrheitlich auf die akustische Präsentation konzentrierten und die visuelle Darbietung ignorierten, so dass der beschriebene Modalitätseffekt hätte auftreten können.

Eine mögliche Alternativerklärung für das Phänomen liefert das phonological loop model von Burgess (1995; Burgess \& Hitch, 1999), das eine Erweiterung des ArbeitsgedächtnisModells von Baddeley (1986; s.o. Abschn. 2.3.1) darstellt. Burgess geht davon aus, dass durch Rehearsal-Prozesse drei Informationsformen in unterschiedlichen Gedächtnis-Knoten kodiert werden: Iteminformationen, phonologische Informationen und Kontextinformationen. Bei der Präsentation einer Lernliste werden nun für jeden Stimulus Phonem-Item-, KontextItem- und Item-Phonem-Assoziationen gebildet. Das Kontextsignal ist eine Art moving win- 
dow, das aus einem Set von zeitlichen Schwingungen (set of temporal oscillators) besteht ( $\mathrm{Ng}$ \& Maybery, 2002, S. 393). Für Listen mit unverbundenem Material wird nur ein Set von Schwingungen angenommen, welche bei der Präsentation der Liste in Form einer Aktivationswelle über den sequentiellen Listenverlauf führt. Bei kumulativem Rehearsal bzw. in der Abrufphase wird diese Aktivationswelle reinitiiert und als Abruf-Cue genutzt.

Die geringen Reproduktionswahrscheinlichkeiten später Listenitems bei „Dominantcumulative"-Rehearsern könnten dieser Annahme zufolge durch einen Abbruch der Aktivationswelle zustande kommen. Da bei fehlender Durchmischung der Rehearsal-Sets für jedes Item nur wenige assoziative Hinweisreize vorhanden sind, würde es dadurch zu einem Verlust des für die Reproduktion eines Items relevanten Abruf-Cues kommen. Selbst bei einer intakten Aktivationswelle nimmt deren Stärke mit der Zeit ab, wenn sie nicht durch Rehearsal neu initiiert und aufgefrischt wird. Da der Abruf früher Listenitems mit dem später Listenitems interferiert, verblasst das Kontextsignal nicht wiedergegebener Items mit der Zeit, und die Reproduktionswahrscheinlichkeit der (noch nicht genannten) Items sinkt.

Für die Annahme einer Rehearsal-basierten Aktivationswelle in der Abrufphase spricht auch das mehrfach gebogene Muster in den seriellen Positionskurven. Das sowohl für die Einzellisten als auch für die gemittelten Werte gefundene multipel gezackte Muster (s.o. Abbildung 5.6) lässt vermuten, dass es durch die Reinitierung der Rehearsal-Aktivationswelle zu Primacy- und Recency-Effekten innerhalb der Rehearsal-Sets in der Abrufsequenz kommt. Ähnliche Zick-Zack-Muster wurden in früheren Arbeiten nur vereinzelt und weniger ausgeprägt gefunden (z.B. Ornstein, Naus \& Stone, 1977).

Ebenfalls stimmig mit der formulierten Interpretation sind die relativ zu den „Noncumulative"-Rehearsern hohen Werte im Ausmaß an subjektiver Organisation bei Kindern, die kumulatives Rehearsal nutzten (s. Tabelle 5.9). Durch die Reinitiierung der Aktivationswelle werden die Items entsprechend der Abfolge der temporal oscillators genannt. Bei kumulativen Rehearsern, die ein neues Rehearsal-Set mit dem ersten Item beginnend nach der seriellen Präsentationsabfolge aufbauen, entspricht diese Abfolge der seriellen Präsentationsabfolge und führt damit zu einem stärkeren Ausmaß an subjektiver Organisation in der Abrufphase.

Durch das wiederholte Memorieren einer festen Itemabfolge wird auch die Assoziativität zwischen diesen Items erhöht, so dass die für den Abruf eines Items notwendigen Aktivationsprozesse (genauer: Such- und Rekonstruktionsprozesse; vgl. Cowan et al., 2000), eine höhere Geschwindigkeit haben. Auf diese Weise sind auch die signifikanten Unterschiede der 
„Within-Set“"- und „Between-Set“"-Latenzen von kumulativen Rehearsern zu erklären (s.o. Abbildung 5.8).

Die Überprüfung der Vorhersagen der Strategie-Emergenz-Theorie (Hasselhorn, 1996) über die Bedeutung metakognitiver Aspekte als zentrale Determinanten des effizienten Strategieerwerbs konnte die von Hasselhorn (1996; Hasselhorn \& Lindner-Müller, 1995) formulierten Annahmen nur teilweise bestätigen. Die erste aus der Strategie-Emergenz-Theorie abgeleitete Vorhersage postuliert eine Zunahme des metamemorialen Wissens für RehearsalProzesse zwischen 9 und 10 Jahren. Das Eintreten der psychologischen Vorhersage auf Gruppenebene führt zu einer ersten Bestätigung der psychologischen Hypothese.

Genauere Betrachtungen zeigten, dass sich eine signifikante Zunahme des strategiespezifischen Metagedächtnisses nur bei der Gruppe von n=10 Kindern nachweisen ließ, die zum vierten Messzeitpunkt erstmals als „Dominant-cumulative“-Rehearser klassifiziert worden waren. Auffallend ist dabei, dass diese Gruppe beim dritten Messzeitpunkt den niedrigsten Wert für das strategiespezifische Metagedächtnis hatte $(M=2.5)$. Der beim vierten Messzeitpunkt erreichte Wert $(M=4.4)$ entspricht dem Wert der für Messzeitpunkt 3 und 4 konsistent als „Dominant-cumulative“-Rehearser $(M=4.52)$ klassifizierten Probanden. Die damit gefundene Parallelität in der Zunahme des metamemorialen Wissens über Rehearsal und deren Anwendung ist eine deutliche Bestätigung für die Annahme Hasselhorns (1996; Hasselhorn \& Grube, im Druck), dass Kinder mit etwa 10 Jahren ihr deklaratives Metagedächtnis auf bislang unreflektiert ausgeführte strategische Lernaktivitäten anwenden und dass sich hieraus eine bewusste Nutzungsmöglichkeit (Emergenz) der Gedächtnisstrategie(n) herausbildet.

Die zweite psychologische Vorhersage, nach der sich bei den zehnjährigen Viertklässlern eine deutlich größere Anzahl von „Dominant-cumulative“-Rehearsern (moderates Strategen-Kriterium) bzw. Rehearsal-Strategen (strenges Strategen-Kriterium; vgl. Hasselhorn \& Lindner-Müller, 1995) finden lassen sollte, konnte nur deskriptiv bestätigt werden. Die inferenzstatistische Überprüfung der Unterschiede erbrachte keinen signifikanten Anstieg in dem Anteil der als Rehearsal-Strategen klassifizierten Kinder im Wechsel von der dritten zur vierten Klasse. Damit hat sich die entsprechende psychologische Vorhersage nicht bestätigt. Allerdings lag zwischen den beiden Erhebungszeitpunkten nur ein halbes Jahr. Frühere Prüfversuche der Vorhersagen der Strategie-Emergenz-Theorie (z.B. Hasselhorn, 1996; Hasselhorn \& Lindner-Müller, 1995) haben dagegen wenigstens einen Abstand von einem Jahr zwischen den untersuchten Klassenstufen realisiert. Es ist daher nicht verwunderlich, dass die 
vergleichsweise strenge Prüfung der zweiten psychologischen Vorhersage in der vorliegenden Untersuchung zu einem negativen Resultat geführt hat.

Weitaus schwerwiegender ist das völlige Fehlen eines Zusammenhangs zwischen dem strategiespezifischen Metagedächtnis und dem strategischen Verhalten auf beiden Klassenstufen. Obwohl die „Dominant-cumulative“-Rehearser in dem betrachteten Zeitraum eine deutliche Steigerung des korrelativen Zusammenhangs zwischen Metagedächtnis und SetGröße aufweisen, konnten keine signifikanten Differenzen in den Korrelationskoeffizienten bzw. deren Veränderungen verzeichnet werden. Dieser Befund spricht auf den ersten Blick gegen die Annahme, dass die alterskorrelierte Zunahme des strategiespezfischen Metagedächtnisses die Ursache des strategischeren Verhaltens älterer Kinder darstellt. Auf der Basis dieses Befundes und der zuvor berichteten Parallelität in der Zunahme des metamemorialen Wissens über Rehearsal und deren Anwendung wäre auch ein umgekehrter Ursache-FolgeZusammenhang denkbar.

Berücksichtigt man wiederum den geringen zeitlichen Abstand zwischen den Erhebungszeitpunkten und die Annahme, dass metakognitive Kenntnisse und (strategische) Regulationsprozesse bidirektional miteinander verflochten sind (Hasselhorn \& Grube, im Druck; Kuhn, 2000; s. Abschn. 2.3.3), so könnte sich die Ausbildung der Emergenz strategischen Verhaltens durchaus über einen längeren Zeitraum erstrecken bzw. später entwickeln, als es von Hasselhorn und Lindner-Müller (1995) postuliert wird. Demnach ist es durchaus plausibel anzunehmen, dass die bewusste Nutzungsmöglichkeit strategischer Prozesse sich erst allmählich herausbildet und nicht auf das enge Zeitfenster zwischen 9 und 10 Jahren beschränkt ist.

Die vierte Vorhersage bezieht sich auf den Zusammenhang zwischen kumulativem Rehearsal und Reproduktionsleistung. Auf beiden Klassenstufen zeigten sich die RehearsalStrategen (strenges Kriterium) in ihrer Lernleistung den Nichtstrategen signifikant überlegen, womit die psychologische Vorhersage bestätigt ist. Dieser Effekt fiel erwartungsgemäß auf der vierten Klassenstufe mit $t(78)=2.76$ stärker aus als auf der dritten Klassenstufe $(t(78)=1.92)$.

Angesichts des Befundmusters ist es fraglich, ob die von Hasselhorn (1996) für die Strategie des kategorialen Organisierens postulierten Zeitangaben generell auf RehearsalProzesse übertragbar sind. Insgesamt sprechen aber die meisten Befunde dafür, dass die Strategie-Emergenz-Theorie einen guten Erklärungsrahmen für die Aneignung und zunehmende Effizienz kumulativer Rehearsal-Prozesse darstellt. Kritisch anzumerken ist jedoch, dass bislang noch kein standardisiertes Verfahren zur Erfassung des metamemorialen Strategiewis- 
sens über Rehearsal-Prozesse existiert und dass somit eine reliable und valide Prüfung der aus der Strategie-Emergenz-Theorie abgeleiteten Vorhersagen nur eingeschränkt möglich ist.

Hasselhorn (1996, S. 174f.) spricht kapazitiven Aspekten bei der strategischen (d.h. bewussten und effizienten) Nutzung kategorialen Organisierens nur eine untergeordnete Bedeutung zu, da sie eher zur Perfektionierung, nicht aber zum Erwerb einer mnemotechnischen Kompetenz beitragen. Weiterhin unterstützt die funktionale Gedächtniskapazität automatische und bewusst-strategische Prozesse gleichermaßen. Die funktionale Gedächtniskapazität hat jedoch durchaus einen Einfluss auf die Anzahl von Items, die pro Zeiteinheit memoriert und kontrolliert werden können, so dass kapazitive Aspekte zumindest beim Erwerb kumulativen Rehearsals eine Rolle spielen können. Wenn die Nutzung kumulativer Memorierprozesse automatisiert bzw. perfektioniert ist, sollte die strategische Komponente aus der Sicht der Strateige-Emergenz-Theorie (Hasselhorn, 1996) jedoch im Vordergrund stehen und sollten kapazitive Aspekte an Bedeutung für die Reproduktionsleistung verlieren.

Wie korrelative Analysen auf Gruppenebene zeigten, hatte die Arbeitsgedächtniskapazität erst beim zweiten und dritten Messzeitpunkt (Klasse 3) einen substantiellen Einfluss auf die Rehearsal-Set-Größe und die Reproduktionsleistung. Dieser Einfluss schien zumindest beim Zusammenhang von Lernleistung und Zahlenspanne rückwärts beim vierten Erhebungszeitpunkt (Klasse 4) wieder deutlich zurückzugehen, was im Sinne der Strategie-EmergenzTheorie als Hinweis darauf interpretiert werden kann, dass die strategische Komponente zwischen 9 und 10 Jahren an Einfluss gewinnt und somit kapazitive Aspekte eher in den Hintergrund treten. Der Einfluss der Gedächtniskapazität auf die Rehearsal-Set-Größe war jedoch auch in der vierten Klassenstufe unvermindert.

Differentielle Betrachtungen zwischen den „Dominant-cumulative“-Rehearsern und den nicht als „Dominant-cumulative“-Rehearser klassifizierten Kindern konnten jedoch systematische Unterschiede zwischen diesen beiden Gruppen aufdecken. Für die letzte Gruppe zeigten sich nahezu keine statistisch bedeutsamen Zusammenhänge zwischen kapazitiven Maßen und der Lernleistung bzw. dem Strategiemaß (Set-Größe). Bei der Gruppe der „Dominantcumulative"-Rehearser fand sich der erwartete Einfluss primär kapazitiver Aspekte (erfasst über die Zahlenspanne vorwärts) beim Erwerb kumulativen Rehearsals. Darüber hinaus konnte eine deutliche Einwirkung von Exekutivfunktionen (erfasst über die Zahlenspanne rückwärts) in einem späteren Stadium der effizienten Nutzung kumulativen Rehearsals (erfasst über den korrelativen Zusammenhang von Set-Größe und Reproduktionsleistung) registriert werden. Danach scheint die Fähigkeit, zwischen Suchräumen hin- und herzuschalten und In- 
formationen aus der Wissensbasis abzurufen bzw. manipulieren zu können, ein zentraler Aspekt der Perfektionierung kumulativen Rehearsals zu sein.

Aus den bisherigen Ausführungen kann gefolgert werden, dass die Bedeutung kapazitiver Aspekte für den Erwerb und Gebrauch kumulativen Rehearsals und die damit einhergehende Frage nach dem kapazitiven Einfluss auf die Gedächtnisleistung erst durch differenzierte Entwicklungsanalysen verstehbar wird. Insgesamt ist die Annahme eines dynamischen Wechselspiels verschiedener Funktionen des Arbeitsgedächtnisses bei der Strategieakquisition und -anwendung plausibel. Die Zusammenhänge erscheinen jedoch für das Rehearsal komplexer zu sein, als es in der Strategie-Emergenz-Theorie für den Erwerb der Organisationsstrategie vorhergesagt wird (vgl. Hasselhorn, 1996, S. 174f.).

In der vorliegenden Untersuchung konnten deutliche Belege für eine Nutzungsineffizienz bei kumulativem Rehearsal entdeckt werden. Im Gegensatz zu neueren Befunden für die Organsiationsstrategie (z.B. Schlagmüller \& Schneider, 2002) stützen die Ergebnisse die Annahme vom Ausbleiben positiver Leistungskonsequenzen beim ersten spontanen bzw. dominanten Strategiegebrauch für das kumulative Rehearsal. Aus dem Eintreten der formulierten psychologischen Vorhersage resultiert somit die Bestätigung der psychologischen Hypothese.

Überraschend war, dass der Anteil von ,ineffizienten“ Probanden sich über die Messzeitpunkte erhöhte. Dies steht im Gegensatz zu der Annahme, dass eine Nutzungsineffizienz häufiger in der Phase der Strategieimplementierung gefunden wird (z.B. Bjorklund et al., 1997; Schneider \& Sodian, 1997). Allerdings verweist Miller (2000) darauf, dass die Nutzungsineffizienz möglicherweise kein allgemeines Phänomen darstellt, sondern nur bei bestimmten Strategien oder Aufgaben auftritt. Bedenkt man weiter, dass die Überprüfung der Nutzungsineffizienz-Hypothese bislang fast ausschließlich an der Organisationsstrategie erfolgt ist, so können die berichteten Ergebnisse nur einen ersten Schritt in der Diskussion über das Auftreten oder Ausbleiben einer Nutzungsineffizienz bei Rehearsal darstellen.

Einen potenziellen Grund für das vermehrte Auftreten ineffizienter Strategienutzung in der späteren Phase der Grundschulzeit stellt der berichtete Zusammenhang zwischen zentralexekutiven Funktionen und der Qualität der Rehearsal-Prozesse dar. Möglicherweise lassen sich bei ineffizienten Strategen Mängel in den relevanten Funktionen feststellen, die für die Koordination der Rehearsal-Sets und die Ressourcenallokation verantwortlich sind. Obwohl keine explizite Überprüfung dieser Vermutung erfolgte, stützen die gefundenen tendenziellen Unterschiede in den Maßen der Gedächtniskapazität zwischen den beiden Gruppen von Aufsteigern/Strategieentdeckern die Annahme, dass der kapazitiven Komponente eine zentrale 
Position bei der Suche nach möglichen Einflussfaktoren bzw. Prädiktoren des effizienten vs. ineffizienten Strategiegebrauchs gebührt.

Wären diese Unterschiede mithilfe eines weniger konservativen einseitigen t-Tests geprüft worden, so hätte der statistisch signifikante Befund an dieser Stelle zu einer deutlicheren Aussage hinsichtlich der Ursachen von Nutzungsineffizienz geführt. Im Gegensatz dazu fiel die Überprüfung von metakognitiven Faktoren oder eines mangelnden Zusammenhangs zwischen Strategieanwendung und Reproduktionsleistung eindeutig aus. Beide Aspekte konnten als Ursache für die gefundenen Leistungsunterschiede zwischen „effizienten“ und „ineffizienten Aufsteigern/Strategieentdeckern“" ausgeschlossen werden.

\subsection{Einordnung der Ergebnisse in Befunde und Modelle der ent- wicklungspsychologischen Gedächtnisforschung}

Die im Rahmen dieser Arbeit zur Entwicklung von Rehearsal im Grundschulalter angestellten Überlegungen verdeutlichen, dass die Entwicklung der Memorierstrategie ein komplexer Vorgang ist und dass „our understanding of strategy development based on crosssectional studies and the analysis of group data does not tell us the whole story about individual developmental trends" (Schneider, 2000, S. 414). Die berichteten Ergebnisse bestätigen zwar viele auf der Grundlage von Altersvergleichen erworbenen Erkenntnisse, korrigieren das bisherige Bild jedoch auch teilweise und erweiteren es durch differentielle Entwicklungsanalysen und die Berücksichtigung von Bedingungsfaktoren des strategischen Verhaltens.

Bestätigt werden konnten die von Ornstein berichteten qualitativen Veränderungen in den Rehearsal-Prozessen, die sich im Verlauf der späten Grundschuljahre vollziehen, und ihre Bedeutung für den alterskorrelierten Anstieg der Gedächtnisleistung. Prinzipiell bestätigt werden konnte auch die von Kunzinger (1985) formulierte Annahme großer Stabilitäten in diesen Entwicklungsprozessen. Allerdings lag das Ausmaß an Stabilität deutlich unter dem vermuteten Maß.

Die zeitgleich gefundene große Variabilitität in den Verlaufsdaten der Strategieimplementierung zeigt weiterhin, dass es nicht den Weg der kognitiven Entwicklung gibt. Die Koexistenz von allgemeinen Entwicklungsschüben und alterskorrelierten Veränderungen interindividueller Differenzen und intraindividueller Verhaltensvariabilitäten in dem beobachteten Zeitraum verdeutlicht einmal mehr die Notwendigkeit einer differentiellen Entwicklungspsychologie (vgl. Hasselhorn, 1995) zur Erfassung funktionaler Entwicklungsaspekte.

Die berichteten Abrufphänomene leisten einen Beitrag zur Frage der Lokation und Form von Rehearsal-Prozessen (vgl. Brown \& Hulme, 1995) und lassen Zweifel daran auf- 
kommen, dass Rehearsal als reine Enkodierstrategie nur einen Einfluss auf die Quantität der reproduzierbaren Informationen hat. Die Befunde stützen hier die Vermutung eines, wenn auch indirekten, Einflusses von Rehearsal auf die Form bzw. Organisationsstruktur der Reproduktionsleistung.

Neben der kritischen Prüfung querschnittlicher Befunde an einer größeren Longitudinalgruppe mit mehreren Erhebungszeitpunkten und einer gegenüber früheren Studien erhöhten Frequenz der Messzeitpunkte (vgl. Ornstein, 1999) war es ein zentrales Ziel der vorliegenden Studie, die in letzter Zeit diskutierten Antezedenzien strategischen Verhaltens für die Rehearsal-Strategie zu untersuchen. Die in diesem Zusammenhang erfolgte Überprüfung der Vorhersagen der Strategie-Emergenz-Theorie (Hasselhorn, 1996) untermauert die Notwendigkeit der Berücksichtigung von metamemorialem Wissen bei der Erforschung strategischer Performanz.

Angesichts der lediglich annähernd befriedigenden internen Konsistenz des Messinstruments und des „Pioniercharakters“ der Studie in diesem Bereich scheint zwar eine vorsichtig-zurückhaltende Interpretation der Daten angebracht, die Befunde sprechen jedoch insgesamt für die Brauchbarkeit der Strategie-Emergenz-Theorie als Erklärungsrahmen für die Emergenz von Rehearsal-Prozessen.

Dringend Nachholbedarf besteht diesbezüglich bei der Entwicklung standardisierter Verfahren zur Erfassung von metamemorialer Bewusstheit von Rehearsal. Es ist kaum nachvollziehbar, dass nahezu die gesamte Forschung zur Metakognition diese Facette strategischen Verhaltens ignoriert und nur das kategoriale Organisieren als Mnemotechnik berücksichtigt hat.

Nicht nur metamemoriale Aspekte, sondern auch kapazitive Faktoren haben einen Einfluss auf die Form und Qualität von Rehearsal. Es konnte gezeigt werden, dass neben der reinen Verarbeitungskapazität und -geschwindigkeit, die bei der Implementierung kumulativen Rehearsals eine Rolle spielen, auch zentralexekutiven Faktoren eine große Bedeutung für die Perfektionierung und damit für die Verarbeitungseffizienz dieser kognitiven Operationen zukommt.

Die kontrovers diskutierte Frage nach den positiven Leistungskonsequenzen strategischen Verhaltens ist in der vorliegenden Arbeit erstmals für Rehearsal untersucht worden. Auch hier kann vor dem Hintergrund fehlender Vergleichsstudien nur eine vorsichtige Antwort gegeben werden. Sie fällt für die Frage nach der generellen Existenz einer Nutzungsineffizienz für Rehearsal positiv aus. Es scheint sich hier jedoch nicht um ein zwingendes Entwicklungsereignis zu handeln. Bei der Diskussion von Prädiktoren für die effiziente vs. inef- 
fiziente Nutzung kumulativen Rehearsals und für das vermehrte Auftreten ineffizienten Strategiegebrauchs in der späteren Grundschulzeit kommt vor allem kapazitiven Faktoren eine entscheidende Funktion zu.

Auch fast zwanzig Jahre nachdem Flavell (1985, S. 218) Rehearsal-Prozesse als ,illdefined group of memory strategies“ bezeichnet hat, ist die Befundlage zum Erwerb und effizienten Gebrauch dieser zentralen Mnemetechnik vergleichsweise dürftig. Es ist zu hoffen, dass sich die entwicklungspsychologische Gedächtnisforschung wieder vermehrt diesen Gedächtnisaktivitäten zuwendet. Spannende und herausfordernde Forschungsfragen gibt es sicherlich eine ganze Reihe. 


\section{Zusammenfassung}

Unter dem Begriff Rehearsal werden in der kognitiven Entwicklungspsychologie Gedächtnisaktivitäten verstanden, denen ein innerer (oder lauter) Memorier- bzw. Wiederholprozess der zu lernenden Inhalte zugrunde liegt. Erste Anzeichen für inneres Wiederholen sind einfache Objektbenennungen (Labeling), gefolgt von Ein-Wort-Wiederholungen (passive rehearsal) und aktiven, größere Informationsmengen umfassenden Memorierprozessen (cumulative rehearsal). Letztere zeichnen sich durch das Wiederholen unterschiedlicher Items innerhalb einer funktionalen Wiederholungseinheit (Rehearsal-Set) aus (vgl. Weinert \& Schneider, 1996). Im Verlauf der Grundschulzeit kann dabei eine Entwicklung von relativ passiven zu aktiveren Rehearsal-Prozessen beobachtet werden, die mit einer Verbesserung der Behaltensleistung verbunden ist (vgl. Ornstein \& Naus, 1985).

Die meisten Befunde zu dieser Entwicklung basieren auf Altersvergleichen und experimentellen Studien der Arbeitsgruppe um Ornstein aus den siebziger und achtziger Jahren. Bislang existieren jedoch keine Untersuchungen über individuelle Entwicklungsverläufe und die Ausbildung der bewussten Nutzungsmöglichkeit (Emergenz) dieser als prototypisch geltenden Gedächtnisstrategie.

Auf der Grundlage eines kombinierten Querschnitt-Längsschnitt-Designs wurden in der vorliegenden Arbeit zentrale Annahmen zur Entwicklung von Rehearsal-Prozessen und ihrem Zusammenhang zur Reproduktionsleistung überprüft. Die Ergebnisse zeigen, dass diese Entwicklung durch eine Komplexität und Differenziertheit der individuellen Entwicklungspfade und nicht durch einen kontinuierlich-gradlinigen Verlauf gekennzeichnet ist.

Zwar konnten analog zu früheren Befunden alterskorrelierte Zuwächse in der Reproduktionsleistung und der mittleren Rehearsal-Set-Größe sowohl beim querschnittlichen Altersvergleich als auch bei Entwicklungsanalysen auf Gruppenebene bestätigt werden, die allerdings bei den Viert- und Sechsklässlern der Querschnittstichprobe und bei der Longitudinalgruppe zum dritten Messzeitpunkt (3. Klasse) nicht mit korrespondierenden Steigerungen im Zusammenhang dieser beiden Faktoren einhergingen. Es fanden sich Entwicklungsstabilitäten des strategischen Verhaltens und der Gedächtnisleistung. Diese erreichten jedoch nicht das Ausmaß wie bei Kunzinger (1985).

Die intraindividuelle Entwicklung des mnemonischen Verhaltens war von drei Trends gekennzeichnet: a) ein Anstieg in der Anzahl von Kindern, die mehr als eine Strategie verwendeten, b) ein Anstieg im Anteil an Kindern, die eine Strategie primär, also im Sinne einer Hauptstrategie, einsetzten und c) ein Anstieg an Kindern mit kumulativem Wiederholungs- 
verhalten. Es fanden sich keine Hinweise für einen abrupten Wechsel von Strategieaneignung und -verlust (Achterbahn-Phänomen), aber auch keine Belege für einen streng graduellen Anstieg im strategischen Verhalten, wie ihn die Arbeiten Ornsteins nahe legen. Die große Variabilität in den Verlaufsdaten der Strategieimplementierung zeigte dabei, dass es nicht den Weg der kognitiven Entwicklung gibt.

Bei der Analyse der seriellen Reproduktionskurven konnten verschiedene Abrufphänomene identifiziert werden, die veranschaulichen, dass Rehearsal-Prozesse entgegen früherer Annahmen auch eine Funktion als Abrufstrategie bzw. -hilfe haben können und damit nicht nur die Quantität, sondern auch die Qualität bzw. Form der Reproduktionsleistung beeinflussen.

Die Überprüfung metakognitiver und kapazitiver Aspekte als Determinanten der qualitativen Veränderungen im Gebrauch der Memorierstrategie zeigte, dass beiden Faktoren ein relevanter Einfluss zugesprochen werden kann. Die Befunde stützen die Vermutung Hasselhorns (1996; Hasselhorn \& Grube, im Druck), dass die Strategie-Emergenz-Theorie einen guten Erklärungsrahmen für die Aneignung und zunehmende Effizienz kumulativer Rehearsal-Prozesse darstellt. Der Einfluss kapazitiver Aspekte auf Akquisition und Perfektionierung von Rehearsal erscheint jedoch komplexer zu sein, als es in der Strategie-Emergenz-Theorie für die Organisationsstrategie vorhergesagt wird (vgl. Hasselhorn, 1996, S. 174f.).

Hinsichtlich der Frage, ob ein erstmaliger spontaner Strategiegebrauch des kumulativen Rehearsals ohne einen entsprechenden Leistungsvorteil vonstatten geht, wie es Analysen intraindividueller Veränderungen im Strategiegebrauch des kategorialen Organisierens zeigen, waren die Befunde heterogen. Die Ergebnisse stützen zwar ausdrücklich die Annahme einer Nutzungsineffizienz für Rehearsal, zeigen aber auch, dass sie keine normative Phase der Strategieentwicklung darstellt.

Trotz zahlreicher einschlägiger Arbeiten der Forschergruppe um Ornstein ist unser Wissen über die (intraindividuelle) Entwicklung von Rehearsal, die Determinanten dieser Entwicklung und ihre Konsequenzen weitgehend ungeklärt. Ziel der vorliegenden Arbeit ist es zum einen, die lange Abstinenz in der Erforschung dieser Mnemotechnik zu beenden und zum anderen erste Antworten auf die in den neunziger Jahren in der kognitiven Entwicklungspsychologie aufgeworfenen Fragen zu geben. Aufgrund des Pilotcharakters, den die aktuelle Studie hier hat, können die Antworten sicherlich nur vorläufige Anregungen darstellen. Es bleibt zu hoffen, dass andere Forscher sie auch als Ansto $\beta$ verstehen, Rehearsal-Prozesse (wieder) vermehrt zu erforschen. 


\section{Literaturverzeichnis}

Allik, J. P. \& Siegel, A. W. (1976). The use of the cumulative rehearsal strategy: A developmental study. Journal of Experimental Child Psychology, 21, 316-327.

Andrade, J. (Ed.), (2001). Working memory in perspective. Hove, U. K.: Psychology Press.

Appel, L. F., Cooper, R. G., McCarrell, N., Sims-Knight, J., Yussen, S. R. \& Flavell, J. H. (1972). The development of the distinction between perceiving and memorizing. Child Development, 43, 1365-1381.

Atkinson, R. C. \& Shiffrin, R. M. (1968). Human memory: A proposed system and its control process. In K. W. Spence \& J. T. Spence (Eds.), The psychology of learning and motivation: Advances in research and theory (Vol. 2, pp. 89-195). New York: Academic Press.

Avons, S. E., Wright, K. L. \& Pammer, K. (1994). The word-length effect in probed serial recall. Quarterly Journal of Experimental Psychology, 47A, 207-231.

Awh, E., Jonides, J., Smith, E. E., Buxton, R. B., Frank, L. R., Love, T., Wong, E. C. \& Gmeindl, L. (1999). Rehearsal in spatial working memory: Evidence form neuroimaging. Psychological Science, 10, 433-437.

Baddeley, A. D. (1986). Working memory. Oxford: Clarendon Press.

Baddeley, A. D. (1999). Essentials of Human Memory. Sussex: Psychology Press Ltd.

Baddeley, A. D. (2000). The episodic buffer: A new component of working memory? Trends in Cognitive Science, 11, 417-423.

Baddeley, A. D. (2002). Is working memory still working? European Psychologist, 7, 85-97.

Baddeley, A. D. \& Hitch, G. (1974). Working memory. In G. H. Bower (Ed.), The Psychology of Learning and Motivation, 8, 47-90. New York: Academic Press.

Baker-Ward, L., Ornstein, P. \& Holden, D. J. (1984). The expression of memorization in early childhood. Journal of Experimental Child Psychology, 37, 555-575.

Baumert, J. (1997). Interesse, Lernstrategien und Schulleistungen. Vortrag gehalten beim 26. Potsdamer Kolloquium zur Lern- und Lehrforschung, Universität Potsdam.

Belmont, J. M. \& Butterfield, E. C. (1969). The relations of short-term memory to development and intelligence. In L. P. Lipsitt \& H. W. Reese (Eds.), Advances in child development and behavior (Vol. 4, pp. 29-82). New York: Academic Press.

Belmont, J. M. \& Butterfield, E. C. (1971). What the development of short-term memory is. Human Development, 14, 236-248. 
Belmont, J. M. \& Butterfield, E. C. (1977). The instructional approach to developmental cognitive research. In R. V. Kail \& J. W. Hagen (Eds.), Perspectives on the development of memory and cognition (pp. 437-481). Hillsdale, NJ: Erlbaum.

Bernbach, H. A. (1967). The effect of labels on short-term memory for colors with nursery school children. Psychonomic Science, 7, 149-150.

Beuhring, T. \& Kee, D. (1987). Developmental relationships among metamemory, elaborative strategy use, and associative memory. Journal of Experimental Child Psychology, 44, 377-400.

Bjorklund, D. (1985). The role of conceptual knowledge in the development of organization in children's memory. In C. J. Brainerd \& M. Pressley (Eds.), Basic processes in memory development: Progress in cognitive development research (pp. 103-142). New York: Springer.

Bjorklund, D. F. (1987). How age changes in knowledge base contribute to the development of children's memory: An interpretative review. Developmental Review, 7, 93-130.

Bjorklund, D. F. (2000). Children's thinking: Developmental function and individual differences ( $3^{\text {rd }}$ edition). Belmont, CA: Wadsworth.

Bjorklund, D. F. \& Bjorklund, B. R. (1985). Organization versus item effects of an elaborated knowledge base on children's memory. Developmental Psychology, 21, 1120-1113.

Bjorklund, D. F. \& Coyle, T. R. (1995). Utilization deficiencies in the development of memory strategies. In F. E. Weinert \& W. Schneider (Eds.), Memory performance and competencies: Issues in growth and development (pp. 161-180). Hillsdale, NJ: Erlbaum.

Bjorklund, D. F. \& Douglas, R. N. (1997). The development of memory strategies. In N. Cowan (Ed.). The development of memory in childhood (pp. 201-246). Hove, East Sussex: Psychology Press.

Bjorklund, D. F., Miller, P. H., Coyle, T. R. \& Slawinski, J. L. (1997). Instructing children to use memory strategies: Evidence of utilization deficiencies in memory training studies. Developmental Review, 17, 411-441.

Blöte, A. W., Resing, W. C. M., Mazer, P. \& van Noort, D.A. (1999). Young children's organization strategies on a same-different task: A microgenetic study and a training study. Journal of Experimental Child Psychology, 74, 21-43.

Borkowski, J. G., Carr, M., Rellinger, M. \& Pressley, M. (1990). Self-regulated cognition: Interdependence of metacognition, attributions, and self-esteem. In Jones, B. F. \& Idol, L. (Eds.), Dimensions of thinking and cognitive instruction (pp. 53-92). Hillsdale, NJ: Erlbaum.

Borkowski, J. G., Milstead, M. \& Hale, C. (1988). Components of children's metamemory: Implications for strategy generalization. In F. E. Weinert \& M. Perlmutter (Eds.), Memory development: Universal changes and individual differences (pp. 73-100). Hillsdale, NJ: Erlbaum. 
Borkowski, J. G. \& Turner, L. A. (1990). Transsituational characteristics of metacognition. In W. Schneider \& F. E. Weinert (Eds.), Interactions among aptitudes, strategies, and knowledge in cognitive performance (pp. 159-176). New York: Springer.

Bortz, J. (1993). Statistik für Sozialwissenschaftler (4. Aufl.). Berlin: Springer.

Bousfield, A. K. \& Bousfield, W.A. (1966). Measurement of clustering and of sequential constancies in repeated free recall. Psychological Reports, 19, 935-942.

Bousfield, W. A. (1953). The occurrence of clustering in the recall of randomly arranged sequences. Journal of General Psychology, 49, 229-240.

Bower, G. H. (2000). A brief history of memory research. In E. Tulving \& F. J. M. Craik (Eds.), The Oxford Handbook of Memory (pp. 3-32). Oxford: University Press.

Bradshaw, G. L. \& Anderson, J. R. (1982). Elaborative encoding as an explanation of levels of processing. Journal of Verbal Learning and Verbal Behavior, 21, 165-174.

Bray, N. W., Hersh, R. E. \& Turner, L. A. (1985). Selective remembering during adolescence. Developmental Psychology, 21, 290-294.

Bray, N. W., Huffman, L. F. \& Fletcher, K. L. (1999). Developmental and intellectual differences in self-report and strategy use. Developmental Psychology, 35, 1223-1236.

Bredenkamp, J. (1983). Übersicht. In J. Bredenkamp \& H. Feger (Hrsg.), Hypothesenprüfung (Enzyklopädie der Psychologie, Themenbereich B, Serie Forschungsmethoden der Psychologie, Bd. 5, S. 1-23). Göttingen: Hogrefe.

Brown, A. L., Bransford, J. D., Ferrara, R. A. \& Campione, J. C. (1983). Learning, remembering, and understanding. In J. H. Flavell \& E. M. Markman (Eds.), Handbook of Child Psychology ( Vol. 3, Cognitive Development. 4th ed., pp. 77-166). New York: Wiley.

Brown, G. D. A. \& Hulme, C. (1995). Modeling item length effects in memory span: No rehearsal needed? Journal of Memory \& Language, 34, 594-621.

Brünken, R., Plass, J. L. \& Leutner, D. (2003). Direct measurement of cognitive load in multimedia learning. Educational Psychologist, 38, 53-61.

Burgess, N. (1995). A solvable connectionist model of immediate recall of ordered lists. In G. Treasuno, D. Touretzky, \& T.K. Leen (Eds.), Advances in neural information processing systems (Vol. 7, pp. 51-58). Cambridge, MA: MIT Press.

Burgess, N. \& Hitch, G. J. (1999). Memory for serial order: A network model of the phonological loop and its timing. Psychological Review, 106, 551-581.

Case, R., Kurland, D. M. \& Goldberg, J. (1982). Operational efficiency and the growth of short-term memory span. Journal of Experimental Child Psychology, 33, 386-404.

Cavanaugh, J. C. (1989). The importance of awareness in memory aging. In L. W. Poon, D. C. Rubin \& B. A. Wilson (Eds.), Everyday cognition in adulthood and late life (pp. 416436). Cambridge: Cambridge University Press. 
Ceci, S. J. \& Bruck, M. (1993). Suggestibility of the child witness: A historical review and synthesis. Psychological Bulletin, 113, 403-439.

Ceci, S. J. \& Liker, J. K. (1986). A day at the races: A study of IQ, expertise, and cognitive complexity. Journal of Experimental Psychology: General, 115, 255-266.

Chi, M.T. H. (1978). Knowledge structures and memory development. In R. S. Siegler (Ed.), Children's thinking: What develops? (pp. 73-96). Hillsdale, NJ: Erlbaum.

Chi, M. T. H. (1981). Knowledge development and memory performances. In M. P. Friedman, J. P. Das \& N. O’Connor (Eds.), Intelligence and learning (pp. 221-229). New York: Plenum.

Chi, M. T., Hutchinson, J. E. \& Robin, A. F. (1989). How inferences about novel domainrelated concepts can be constrained by structured knowledge. Quarterly, 35, 27-62.

Cohen, B. H., Sakoda, J. M. \& Bousfield, W. A. (1954). The statistical analysis of the incidence of clustering in the recall of randomly arranged associates. Washington DC: United States Government Printing Office.

Cowan, N. (1992). Verbal memory span and the timing of spoken recall. Journal of Memory and Language, 31, 668-684.

Cowan, N. (1994). Mechanisms in verbal short-term memory. Current Directions in Psychological Science, 3, 185-189.

Cowan, N. (Ed.), (1997). The Development of Memory in Childhood. Hove East Sussex: Psychology Press.

Cowan, N. (1997). The development of working memory. In N. Cowan (Ed.). The Development of Memory in Childhood (pp. 163-199). Hove East Sussex: Psychology Press.

Cowan, N., Nugent, L. D. \& Elliott, E. M. (2000). Memory-search and rehearsal processes and the word length effect in immediate recall: A synthesis in reply to service. Quarterly Journal of Experimental Psychology, 53, 666-670.

Cowan, N., Saults, J. S., Winterowd, C. \& Sherk, M. (1991). Enhancement of 4-year-old children's memory span for phonologically similar and dissimilar word lists. Journal of Experimental Child Psychology, 51, 30-52.

Cox, B. D., Ornstein, P. A., Naus, M. J., Maxfield, D. \& Zimler, J. (1989). Children's concurrent use of rehearsal and organizational strategies. Developmental Psychology, 25, 619627.

Coyle, T. R. \& Bjorklund, D. F. (1997). Age differences in, and consequences of, multipleand variable strategy use on a multitrial sort-recall task. Developmental Psychology, 33, 372-380. 
Coyle, T. R., Read, L. E., Gaultney, J. F. \& Bjorklund, D. F. (1999). Giftedness and variability in strategic processing on a multitrial memory task: Evidence for stability in gifted cognition. Learning and Individual Differences, 10, 273-290.

Craik, F. I. M. \& Lockhart, R. S. (1972). Levels of processing. A framework for memory research. Journal of Verbal Learning and Verbal Behavior, 11, 671-684.

Craik, F. I. M. \& Tulving, E. (1975). Depth of processing and the retention of words in episodic memory. Journal of Experimental Psychology: General, 104, 268-294.

Cuvo, A. J. (1974). Incentive level influence on overt rehearsal and free recall as a function of age. Journal of Experimental Child Psychology, 18, 167-181.

Cuvo, A. J. (1975). Developmental differences in rehearsal and free recall. Journal of Experimental Child Psychology, 19, 265-278.

DeLoache, J. S., Cassidy, D. J. \& Brown, A. L. (1985). Precursors of mnemonic strategies in very young children's memory. Child Development, 56, 125-137.

Ebbinghaus, H. (1897). Über eine neue Methode zur Prüfung geistiger Fähigkeiten und ihrer Anwendung bei Schulkindern. Zeitschrift für Psychologie und Physiologie der Sinnesorgane, 13, 401-459.

Eccles, J. S., Roeser, R., Wigfield, A. \& Freeman-Doan, C. (1999). Academic and motivational pathways through middle childhood. In L. Balter \& C. Tamis-LeMonda (Eds.), Child psychology: A handbook of contemporary issues (pp. 287-317). New York: Garland.

Entwistle, N. J. \& Ramsden, P. (1983). Understanding student learning. London: Croom Helm.

Fernandez-Duque, D., Baird, J. A. \& Posner, M. I. (2000). Executive attention and metacognitive regulation. Consciousness and Cognition, 9, 288-307.

Flavell, J. H. (1970). Developmental studies of mediated memory. In H. W. Reese \& L. P. Lipsitt (Eds.), Advances in child development and behavior (Vol. 5, pp. 181-211). New York: Academic Press.

Flavell, J. H. (1979). Metacognition and cognitive monitoring: A new area of cognitivedevelopmental inquiry. American Psychologist, 34, 906-911.

Flavell, J. H. (1981). Cognitive monitoring. In W. P. Dickson (Ed.), Children's oral communication skills (pp. 35-60). New York: Academic.

Flavell, J. H. (1985). Cognitive development ( $2^{\text {nd }}$ ed.). Englewood Cliffs, NJ: Prentice-Hall.

Flavell, J. H. (1999). Cognitive development: Children's knowledge about the mind. In J. T. Spence, J. M. Darley \& D. J. Foss (Eds.), Annual review of psychology (pp. 21-45). Palo Alto, CA: Annual Review. 
Flavell, J. H., Beach, D. R. \& Chinsky, J. M. (1966). Spontaneous verbal rehearsal in a memory task as a function of age. Child Development, 37, 283-299.

Flavell, J. H., Green, F. L. \& Flavell, E. R. (2000). Development of children's awareness of their own thoughts. Journal of Cognition \& Development, 1, 97-112.

Flavell, J. H., Green, F. L., Flavell, E. R. \& Grossman, J. B. (1997). The development of children's knowledge about inner speech. Child Development, 68, 39-47.

Flavell, J. H. \& Wellman, H. M. (1977). Metamemory. In R. V. Kail \& J. W. Hagen (Eds.), Perspectives on the development of memory and cognition (pp. 3-33). Hillsdale, NJ: Erlbaum.

Folds, T. H., Footo, M. M., Guttentag, R. E. \& Ornstein, P. A. (1990). When children mean to remember: Issues of context specificity, strategy effectiveness, and intentionality in the development of memory. In D. F. Bjorklund (Ed.), Children's strategies: Contemporary views of cognitive development (pp. 67-91). Hillsdale, NJ: Erlbaum.

Footo, M. M., Guttentag, R. \& Ornstein, P. A. (1988). Capacity demands of strategy execution: Effects of training and practice. Paper presented at the annual meeting of the American Educational Research Association, New Orleans.

Frey, K. S. \& Ruble, D. N. (1990). Strategies for comparative evaluation: Maintaining a sense of competence across the life span. In R. J. Stenberg \& K. Lolligian, Jr. (Eds.), Competence considered (Vol. 7; pp. 167-189). New Haven, CT: Yale University Press.

Frick, R. W. (1989). Explanations of grouping in immediate ordered recall. Memory \& Cognition, 17, 551-562.

Garrity, L. I. (1975). An electromyographical study of subvocal speech and recall in preschool children. Developmental Psychology, 11, 274-281.

Gathercole, S. E. (1999). Cognitive approaches to the development of short-term memory. Trends in Cognitive Science, 3, 410-419.

Gathercole, S. E. \& Martin, A. J. (1996). Interactive processes in phonological memory. In S. E. Gathercole (Ed.), Models of short-term memory. Hove East Sussex: Erlbaum .

Gaultney, J. F., Bjorklund, D. F. \& Schneider, W. (1992). The role of children's expertise in a strategic memory task. Contemporary Educational Psychology, 17, 244-257.

Gerjuoy, I. R. \& Spitz, H. H. (1966). Associative clustering in free recall: Intellectual and developmental variables. American Journal of Mental Deficiency, 70, 918-927.

Goldin-Meadow, S. (2001). Giving the mind a hand: The role of gesture in cognitive change. In J. L. McClelland \& R. S. Siegler (Eds.), Mechanisms of cognitive development: Behavioral and neural perspectives. Carnegie mellon symposia on cognition (pp. 5-31). Mahwah, N. J.: Lawrence Erlbaum Associates, Inc.

Goswami, U. (1998). Cognition in children. Hove, East Sussex: Psychology Press. 
Goswami, U. (2001). So denken Kinder. Einführung in die Psychologie der kognitiven Entwicklung. Bern: Huber.

Guttentag, R. E. (1984). The mental effort requirement of cumulative rehearsal: A Developmental Study. Journal of Experimental Child Psychology, 37, 92-106.

Guttentag, R. E. \& Lange, G. (1994). Motivational influences on children's strategic remembering. Learning and Individual Differences, 6, 309-330.

Guttentag, R. E., Ornstein, P. A. \& Siemens, L. (1987). Children's spontaneous rehearsal: Transitions in strategy acquisition. Cognitive Development, 2, 307-326.

Hagen, J. W., Hargrave, S. \& Ross, W. (1973). Prompting and rehearsal in short-term memory. Child Development, 44, 201-204.

Hagen, J. W. \& Kingsley, P. R. (1968). Labeling effects in short-term memory. Child Development, 39, 113-121.

Hagen, J. W., Meacham, J. A. \& Mesibov, G. (1970). Verbal learning, rehearsal, and shortterm memory. Cognitive Psychology, 1, 47-58.

Hagen, J. W. \& Stanovich K. G. (1977). Memory: Strategies of acquisition. In R. V. Kail \& J. W. Hagen (Eds.), Perspectives on the development of memory and cognition (pp. 89111). Hillsdale, NJ: Erlbaum.

Hager, W. (1992a). Jenseits von Experiment und Quasi-Experiment. Zur Struktur psychologischer Versuche und zur Ableitung von Vorhersagen. Göttingen: Hogrefe.

Hager, W. (1992b). Eine Strategie zur Entscheidung über psychologische Hypothesen. Psychologische Rundschau, 43(1), 18-29.

Hager, W. \& Hasselhorn, M. (Hrsg.), (1994). Handbuch deutschsprachiger Wortnormen. Göttingen: Hogrefe.

Hager, W. \& Hasselhorn, M. (1995). Testing psychological hypotheses addressing two independent and one dependent variables. Perceptual and Motor Skills, 81, 1171-1182.

Hale, S. (1990). A global developmental trend in cognitive processing speed. Child Development, 61, 653-663.

Harnishfeger, K. K. \& Bjorklund, D. F. (1990). Children's strategies: A brief history. In D. F. Bjorklund (Ed.), Children's strategies: Contemporary views of cognitive development. Hillsdale, NJ: Erlbaum.

Hasselhorn, M. (1988). Wie und warum verändert sich die Gedächtnisspanne über die Lebensspanne? Zeitschrift für Entwicklungspsychologie und Pädagogische Psychologie, 20, 322-337.

Hasselhorn, M. (1992). Metakognition und Lernen. In G. Nold (Ed.), Lernbedingungen und Lernstrategien: Welche Rolle spielen kognitive Verstehensstrukturen? (S. 35-63). Tübingen: Gunter Narr. 
Hasselhorn, M. (1994). Zur Erfassung von Metagedächtnisaspekten bei Grundschulkindern. Zeitschrift für Entwicklungspsychologie und Pädagogische Psychologie, 26, 71-78.

Hasselhorn, M. (1995). Beyond production deficiency and utilization inefficiency: Mechanisms of the emergence of strategic categorization in episodic memory tasks. In F. E. Weinert \& W. Schneider (Eds.), Memory development and competencies: Issues in growth and development (pp. 141-159). Mahwah, NJ: Erlbaum.

Hasselhorn, M. (1996). Kategoriales Organisieren bei Kindern: Zur Entwicklung einer Gedächtnisstrategie. Göttingen: Hogrefe.

Hasselhorn, M. (2001). Metakognition. In D. H. Rost (Hrsg.), Handwörterbuch Pädagogische Psychologie (2. Aufl., S. 466-471). Weinheim: Beltz, PVU.

Hasselhorn, M. \& Grube, D. (im Druck). Gedächtnisentwicklung (Grundlagen). In W. Schneider \& B. Sodian (Hrsg.), Enzyklopädie der Psychologie, Serie Entwicklungspsychologie, Band 2: Kognitive Entwicklung. Göttingen: Hogrefe.

Hasselhorn, M., Hager, W. \& Baving, L. (1989). Zur Konfundierung metakognitiver und motivationaler Aspekte im Prädiktionsverfahren. Zeitschrift für experimentelle und angewandte Psychologie, 36, 31-41.

Hasselhorn, M., Jaspers, A. \& Hernando, M.-D. (1990). Typizitätsnormen zu zehn Kategorien für Kinder von der Vorschule bis zur vierten Grundschulklasse. Sprache \& Kognition, 9, 92-108.

Hasselhorn, M. \& Lindner-Müller, C. (1995). Kategoriales Organisieren und kumulatives Rehearsal: Zur Entwicklung der kombinierten Nutzung zweier Gedächtnisstrategien. Zeitschrift für Entwicklungspsychologie und Pädagogische Psychologie, 27, 139-156.

Hasselhorn, M., Mähler, C. \& Grube, D. (1995). Entwicklungsveränderungen und -stabilitäten im Metagedächtnis während der Grundschuljahre. Empirische Pädagogik, 9, 33-53.

Hasselhorn, M., Richter, M. \& Lingen, M. (submitted). Developmental and individual Differences in a repeated free recall task: Combined strategy use and its efficiency.

Helmke, A. (1998). Vom Optimisten zum Realisten? Zur Entwicklung des Fähigkeitsselbstkonzeptes vom Kindergarten bis zur 6. Klassenstufe. In F. E. Weinert. (Hrsg.), Entwicklung im Kindesalter (S. 115-132). Weinheim: PVU.

Helmke, A. (1999). From optimism to realism? Development of children's academic selfconcept from kindergarten to grade 6. In F. E. Weinert \& W. Schneider (Eds.), Individual Development from 3 to 12 (pp. 198-221). Cambridge: Cambridge University Press.

Helmke, A. \& Rheinberg, F. (1996). Anstrengungsvermeidung - Morphologie eines Konstruktes. In C. Spiel, U. Kastner-Koller \& P. Deimann (Hrsg.), Motivation und Lernen aus der Perspektive lebenslanger Entwicklung (S. 207-244). Münster: Waxmann. 
Helmke, A. \& Aken, M. A. G. van (1995). The causal ordering of academic achievement and self-concept of ability during elementary school: A longitudinal study. Journal of Educational Psychology, 87, 624-637.

Henry, L. A. \& Millar, S. (1993). Why does memory span improve with age? A review of the evidence of two current hypotheses. European Journal of Cognitive Psychology, 5, 241287.

Henson, R. N., Burgess, N. \& Frith, C. D. (2000). Recoding, storage, rehearsal and grouping in verbal short-term memory: An fMRI study. Neuropsychologia, 38, 426-440.

Hitch, G. J., Burgess, N., Towse, J. N. \& Culpin, V. (1996). Temporal grouping effects in immediate recall: A working memory analysis. Quarterly Journal of Experimental Psychology: Human Experimental Psychology, 49A, 116-139.

Hitch, G. J., Halliday, M. S., Schaafstal, A. M. \& Heffernan, T. M. (1991). Speech, "inner speech," and the development of short-term memory: Effects of picture labeling on recall. Journal of Experimental Child Psychology, 51, 220-234.

Hock, H. S., Park, C. L. \& Bjorklund, D. F. (1998). Temporal organization in children's strategy formation. Journal of Experimental Child Psychology, 70, 187-206.

Howard, M. W. \& Kahana, M. J. (2001). A distributed representation of temporal context. Journal of Mathematical Psychology, 46, 269-299.

Howard, M. W. \& Kahana, M. J. (2002). When does semantic similarity help episodic retrieval? Journal of Memory \& Language, 46, 85-98.

Howe, M. L. \& O'Sullivan, J. T. (1990). The development of strategic memory: Coordinating knowledge, metamemory, and resources. In D. F. Bjorklund (Ed.), Children's strategies: Contemporary views of cognitive development (pp. 129-155). Hillsdale, NJ: Erlbaum.

Hulme, C., Newton, P., Cowan, N., Stuart, G. \& Brown, G. (1999). Think before you speak: pauses, memory search, and trace redintegration processes in verbal memory span. Journal of Experimental Psychology: Learning, Memory, and Cognition, 25, 447-463.

Hulme, C., Roodenrys, S., Schweickert, R., Brown, G. D. A., Martin, S. \& Stuart, G. (1997). Word frequency effects on short-term memory tasks: Evidence for a redintegration process in immediate recall. Journal of Experimental Psychology: Learning, Memory, and Cognition, 23, 1217-1232.

Hulme, C., Thomson, N., Muir, C. \& Lawrence, A. (1984). Speech rate and the development of short-term memory span. Journal of Experimental Child Psychology, 38, 241-253.

James, W. (1890). The principles of psychology (Bd. 1 und 2). New York: Holt.

Jonston, R. S. \& Conning, A. (1990). The effects of overt and covert rehearsal on the emergence of the phonological similarity effect in 5-year-old children. British Journal of Developmental Psychology, 5, 243-248. 
Justice, E. M. (1985). Categorization as a preferred memory strategy: Developmental changes during elementary school. Developmental Psychology, 21, 1105-1110.

Justice, E. M. (1986). Developmental changes in judgments of relative strategy effectiveness. British Journal of Developmental Psychology, 4, 75-81.

Kail, R. V. (1991). Development of processing speed in childhood and adolescence. In H. W. Reese (Ed.), Advances in child development and behavior (pp. 490-501). New York: Academic Press

Kail, R. V. \& Salthouse, T. A. (1994). Processing speed as a mental capacity. Acta Psychologica, 86, 199-225.

Kee, D. W. \& Davis, L. (1988). Mental effort and elaboration. A developmental analysis. Contemporary Educational Psychology, 13, 221-228.

Kee, D. W. \& Davis, L. (1990). Mental effort and elaboration: Effects of accessibility and instruction. Journal of Experimental Child Psychology, 49, 264-274.

Keeney, T. J., Cannizzo, S. R. \& Flavell, J. H. (1967). Spontaneous and induced verbal rehearsal in a recall task. Child Development, 38, 952-966.

Kellas, G., McCauley, C. \& McFarland Jr., C. E. (1975a). Developmental aspects of storage and retrieval. Journal of Experimental Child Psychology, 19, 51-62.

Kellas, G., McCauley, C. \& McFarland Jr., C. E. (1975b). Reexamination of externalized rehearsal. Journal of Experimental Psychology: Human Learning and Memory, 104, 8490.

Kennedy, B. A. \& Miller, D. J. (1976). Persistent use of verbal rehearsal as a function of information about its value. Child Development, 57, 566-569.

Kihlstrom, J. F. (1993). What does the self look like? In T. K. Srull \& R. S. Weyer Jr. (Eds.), The mental representation of trait and autobiographical knowledge about the self: Advances in social cognition (Vol. 5, pp. 79-90). Hillsdale, NJ: Erlbaum.

Kingsley, P. R. \& Hagen, J. W. (1969). Induced versus spontaneous rehearsal in short-term memory in nursery school children. Developmental Psychology, 1, 40-46.

Koriat, A. (2000). The feeling of knowing: Some metatheoretical implications for consciousness and control. Consciousness and Cognition, 9, 149-171.

Krapp, A. (1997). Selbstkonzept und Leistung - Dynamik ihres Zusammenspiels: Literaturüberblick. In F. E. Weinert \& A. Helmke (Eds.), Entwicklung im Grundschulalter (S. 325-339). Weinheim: Beltz, PVU.

Krauth, J., \& Lienert, G. A. (1973). KFA. Die Konfigurationsfrequenzanalyse und ihre Anwendung in Psychologie und Medizin. Freiburg: Alber.

Kuhn, D. (2000). Metacognitive development. Current Directions in Psychological Science, 9, 178-181. 
Kunzinger, E. L. (1985). A short-term longitudinal study of memorial development during early grade school. Developmental Psychology, 21, 642-646.

Kunzinger, E. L. \& Witryol, S. L. (1984). The effects of differential incentives on secondgrade rehearsal and free recall. The Journal of Genetic Psychology, 144, 19-30.

Kurtz, B. E. \& Weinert, F. E. (1989). Metamemory, memory performance, and causal attributions in gifted and average children. Journal of Experimental Child Psychology, 48, 4561.

Lange, G. \& Pierce, S. H. (1992). Memory-strategie learning and maintenence in preschool children. Developmental Psychology, 28, 453-462.

LeBlanc, R. S., Muise, J. G. \& Blanchard, L. (1992). Backward masking in children and adolescents: Sensory transmission, accrual rate and asymptotic performance. Journal of Experimental Child Psychology, 53, 105-114.

Liberty, C. \& Ornstein, P. A. (1973). Age differences in organization and recall: The effects of training in organization. Journal of Experimental Child Psychology, 15, 169-186.

Lindberg, M. A. (1980). Is knowledge base development a necessary and sufficient condition for memory development? Journal of Experimental Child Psychology, 30, 401-410.

Lindberg, M. (1991). A taxonomy of suggestibility and eyewitness memory: Age, memory process, and focus of analysis. In J. L. Doris (Ed.), The suggestibility of children's recollections. Washington, DC: American Psychological Association.

Locke, J. L. \& Fehr, F. S. (1970). Young children's use of the speech code in a recall task. Journal of Experimental Child Psychology, 10, 367-373.

Marsh, H. W. (1986). Verbal and math self-concepts: An internal/external frame of reference model. American Educational Research Journal, 23 (1), 129 - 149.

Marsh, H. W. (1990). The structure of academic self-concept: The Marsh/Shavelson model. Journal of Educational Psychology, 82, 623-636.

Marsh, H. W., Byrne, B. M. \& Shavelson, R. J. (1988). A multifaceted academic self-concept: Its hierarchical structure and its relation to academic achievement. Journal of Educational Psychology, 80, 366-380.

Marsh, H. W., Ellis, L. A. \& Craven, R. G. (2002). How do preschool children feel about themselves? Unraveling measurement and multidimensional self-concept structure. Developmental Psychology, 38, 376-393.

Marsh, H. W. \& Shavelson, R. J. (1985). Self-Concept: Its multifaceted, hierarchical structure. Educational Psychologist, 20, 107 - 125.

McCombs, B. L. (1986). The role of self-system in self regulated learning. Contemporary Educational Psychology, 11, 314-332. 
McCormick, C. B. \& Pressley, M. (1997). Educational Psychology: Learning, Instruction, Assessment. New York: Addison Wesley Longman.

McGilly, K. \& Siegler, R. S. (1989). How children choose among serial recall strategies. Child Development, 60, 172-182.

McGilly, K. \& Siegler, R. S. (1990). The influence of encoding and strategic knowledge on children's choices among serial recall strategies. Developmental Psychology, 26, 931941.

Medin, D. L. \& Aguilar, C. (1999). Categorization. In R. A. Wilson \& F. C. Keil (Eds.), The MIT encyclopedia of the cognitive sciences. Cambridge, MA: MIT Press.

Meyer, W.-U. (1984). Das Konzept von der eigenen Begabung. Bern: Hans Huber.

Miller, L. T. \& Vernon, P. A. (1997). Developmental changes in speed of information processing in young children. Developmental Psychology, 33, 549-554.

Miller, P. H. (1990). The development of strategies of selective attention. In D. F. Bjorklund (Ed.), Children's strategies: Contemporary views of cognitive development (pp. 157184). Hillsdale, NJ: Erlbaum

Miller, P. H. (1994). Individual differences in children's strategic behavior: Utilization deficiencies. Learning and Individual Differences, 6, 285-307.

Miller, P. H. (2000). How best to utilize a deficiency. Child Development, 71, 1013-1017.

Miller, P. H. \& Seier, W. L. (1994). Strategy utilization deficiencies in children: When, where, and why. In H. W. Reese (Ed.), Advances in Child Development and Behavior (pp. 107-156). New York: Academic Press.

Miyake, A., Friedman, N. P., Emerson, M. J., Witzki, A. H., Howerter, A. \& Wager, T. D. (2000). The unity and diversity of executive functions and their contribution to complex 'frontal lobe' tasks: A latent variable analysis. Cognitive Psychology, 41, 49-100.

Miyake, A. \& Shah, P. (Eds.), (1999). Models of working memory: Mechanisms of active maintenance and executive control. New York: Cambridge University Press.

Moreno, R. \& Mayer, R. E. (2000). A coherence effect in multimedia learning: The case for minimizing irrelevant sounds in the design of multimedia instructional messages. Journal of Educational Psychology, 92, 117-125.

Moses, L. J. \& Baird, J. A. (1999). Metacognition. In R. A. Wilson \& F. C. Keil (Eds.), The MIT encyclopedia of the cognitive sciences. Cambridge, MA: MIT Press.

Murray, D. J. (1967). Overt versus covert rehearsal in short-term memory. Psychonomic Science, $7,363-364$.

Nairne, J. S. (2002). Remembering over the short-term: The case against the standard model. Annual Review of Psychology, 53, 53-81. 
Naus, M. J. \& Ornstein, P. A. (1983). The development of memory strategies: Analysis, questions, and issues. In M. T. H. Chi (Ed.), Trends in memory development research. Contributions to Human Development (Vol. 9, pp. 1-30). Basel: S. Karger.

Naus, M. J. \& Ornstein, P. A. (1985). An investigation of the effects of expertise upon mnemonic strategies. Unpublished manuscript.

Naus, M. J., Ornstein, P. A. \& Aivano, S. (1977). Developmental changes in memory: The effects of processing time and rehearsal instructions. Journal of Experimental Child Psychology, 23, 237-251.

Naus, M. J., Ornstein, P. A. \& Kreshtool, K. (1977). Developmental differences in recall and recognition: The relationship between rehearsal and memory as test expectation changes. Journal of Experimental Child Psychology, 23, 252-265.

Neinmark, E. D. (1976). The natural history of spontaneous mnemonic activity under conditions of minimal experimental constraint. In A. D. Pick (Ed.), Minnesota symposia on child psychology (Vol. 10, pp. 84-118). Minneapolis: University of Minnesota Press.

Nelson T. O. \& Narrens, L. (1990). Metamemory: A theoretical framework and new findings. In G. Bower (Ed.), The psychology of learning and motivation (pp. 125-173). New York: Academic Press.

Newman, L. S. (1990). Intentional and unintentional memory in young children: Remembering vs. playing. Journal of Experimental Child Psychology, 50, 243-258.

Ng, H. L. H. \& Maybery, M. T. (2002). Grouping in short-term verbal memory: Is position coded temporally? The Quarterly Journal of Experimental Psychology, 55A, 391-424.

Nicholls, J. (1979). Development of perception of attainment and causal attributions for success and failure in reading. Journal of Educational Psychology, 71, 94-99.

Nolen, S. B. (1988). Reasons for studying: Motivational orientations and study strategies. Cognition and Instructions, 5, 269-287.

Oka, E. R. \& Paris, S. C. (1987). Patterns of motivation and reading skills in underachieving children. In S. Ceci (Ed.), Handbook of cognitive, social and neurological aspects of learning disabilities (Vol. 2, pp. 115-145). Hillsdale, NJ: Erlbaum.

Ornstein, P. (1999). Comments: Toward an understanding of the development of memory. In F. E. Weinert \& W. Schneider (Eds.), Individual development from 3 to 12: Findings from the Munich Longitudinal Study (pp. 94-105). Cambridge: Cambridge University Press.

Ornstein, P. A., Baker-Ward, L. \& Naus, M. J. (1988). The development of mnemonic skill. In F. E. Weinert \& M. Perlmutter (Eds.), Memory Development: Universal Changes and Individual Differences (pp. 31-51). Hillsdale, N. J.: Lawrence Erlbaum.

Ornstein, P. A. \& Haden, C. A. (2001). Memory Development or the Development of Memory. Current Directions in Psychological Science, 10, 202-205. 
Ornstein, P. A., Medlin, R. G., Stone, B. P. \& Naus, M. J. (1985). Retrieving for Rehearsal: An Analysis of Active Rehearsal in Children's Memory. Developmental Psychology, 21, 633-641.

Ornstein, P. A. \& Naus, M. J. (1978). Rehearsal Processes in children's memory. In P. A. Ornstein (Ed.), Memory Development in Children (pp. 69-99). Hillsdale, N.J.: Erlbaum.

Ornstein, P. A. \& Naus, M. J. (1983). "Rehearsing" according to artificially generated rehearsal patterns: An analysis of active rehearsal. Bulletin of the Psychonomic Society, $21,419-422$.

Ornstein, P. A. \& Naus, M. J. (1985). Effects of the knowledge base on children's memory strategies. Advances in Child Development and Behavior, 19, 113-148.

Ornstein, P. A., Naus, M. J. \& Liberty, C. (1975). Rehearsal and organizational processes in children's memory. Child Development, 46, 818-830.

Ornstein, P. A., Naus, M. J. \& Miller, T. D. (1977). The effects of list organization and rehearsal activity on children's free recall. Child Development, 48, 292-295.

Ornstein, P. A., Naus, M. J. \& Stone, B. P. (1977). Rehearsal training and development differences in memory. Child Development, 13, 15-24.

O'Sullivan, J. T. (1993). Preschoolers' beliefs about effort, incentives, and recall. Journal of Experimental child psychology, 55, 396-414.

O'Sullivan, J. T. \& Howe, M. L. (1998). A different view of metamemory with illustrations from children's beliefs about long-term retention. European Journal of Psychology of Education, 13, 9-28.

Oyen, A. S. \& Bebko, J. M. (1996). The effects of computer games and lesson contexts on children's mnemonic strategies. Journal of Experimental Child Psychology, 62, 173189.

Patterson, K. E., Meltzer, R. H. \& Mandler, G. (1971). Inter-response times in categorized free recall. Journal of Verbal Learning \& Verbal Behavior, 10, 417-426.

Pekrun, R. (1983). Schulische Persönlichkeitsentwicklung. Frankfurt: Lang.

Pierce, S. H. \& Lange, G. (2000). Relationships among metamemory, motivation and memory performance in young school-age children. British Journal of Developmental Psychology, 18, 121-135.

Pintrich, P. R. \& Garcia, T. (1993). Intraindividual differences in students' motivation and self-regulated learning. Zeitschrift für Pädagogische Psychologie, 7, 99-107.

Pollio, H. R., Richards, S. \& Lucas, R. (1969). Temporal properties of category recall. Journal of Verbal Learning \& Verbal Behavior, 8, 529-536.

Pressley, M. (1982). Elaboration and memory development. Child Development, 53, 296-309. 
Pressley, M., Borkowski, J. G. \& O’Sullivan, J. T. (1985). Children's metamemory and the teaching of memory strategies. In D. L. Forrest-Pressley, G. E. MacKinnon \& T. G. Waller (Eds.), Metacognition, cognition, and human performance (Vol. I, pp. 111-153). Orlando, FL: Academic Press.

Pressley, M., Borkowski, J. G. \& Schneider, W. (1987). Cognitive strategies: Good strategy users coordinate metacognition and knowledge. In R. Vasta \& G. Whitehurst (Eds.), Annals of child development (Vol. 5, pp. 89-125) Greenwich, CT: JAI Press.

Pressley, M., Borkowski, J. G. \& Schneider, W. (1989). Good information processing: What is it and what education can do to promote it. International Journal of Educational Research, 13, 857-867.

Pressley, M. \& Levin, J. R. (1977). Developmental differences in subjects' associativelearning strategies and performance: Assessing a hypothesis. Journal of Experimental Child Psychology, 24, 431-439.

Pressley, M. \& Schneider, W. (1997). Introduction to memory development during childhood and adolescence. Mahwah, NJ: Erlbaum.

Rabinowitz, M. \& Chi, M. T. H. (1987). An interactive model of strategic processing. In S. J. Ceci (Ed.), Handbook of cognitive, social and neuropsychological aspects of learning disabilities (Vol. 2, pp. 83-102). Hillsdale, NJ: Erlbaum.

Reese, H. W. (1962). Verbal mediation as a function of age level. Psychological Bulletin, 59, 502-509.

Robbins, M. C. \& Nolan, J. M. (2000). A measure of semantic category clustering in freelisting tasks. Field Methods, 12, 18-28.

Roenker, D. C., Thompson, C. P. \& Brown, S. C. (1971). Comparison of measures for the estimation of clustering in free recall. Psychological Bulletin, 76, 45-48.

Rohwer, W. D. Jr. (1980). An elaborative conception of learner differences. In R. E. Snow, P. A. Federico \& W. E. Montaague (Eds.). Aptitude, learning, and instruction: Vol. 2. Cognitive process analyses of learning and problem. Hillsdale, NJ: Erlbaum.

Ruble, D. N. \& Frey, K. S. (1991). Changing patterns of comparative behavior as skills are acquired: A functional model of self-evaluation. In J. Suls \& A. Wills (Eds.), Social comparison: Contemporary theory research (pp. 70-112). Hillsdale, NJ: Erlbaum.

Rundus, D. (1971). Analysis of rehearsal processes in free recall. Journal of Experimental Psychology, 89, 63-77.

Rundus, D. \& Atkinson, R. C. (1970). Rehearsal processes in free recall: A procedure for direct observation. Journal of Verbal Learning and Verbal Behavior, 9, 99-105.

Sanders, R. E., Murphy, M. D., Schmitt, F. A. \& Walsh, K. K. (1980). Age differences in free recall rehearsal strategies. Journal of Gerontology, 35, 550-558. 
Schiefele, U. (1989). Motivated conditions of text comprehension. Zeitschrift Päodgogik, 34, 687-708.

Schiefele, U. (1996). Motivation und Lernen mit Texten. Göttingen: Hogrefe.

Schlagmüller, M. \& Schneider, W. (2002). The development of organizational strategies in children: Evidence from a microgenetic longitudinal study. Journal of Experimental Child Psychology, 81, 298-319.

Schlagmüller, M., Visé, M. \& Schneider, W. (2001). Zur Erfassung des Gedächtniswissens bei Grundschulkindern: Konstruktionsprinzipien und empirische Bewährung der Würzburger Testbatterie zum deklarativen Metagedächtnis. Zeitschrift für Entwicklungspsychologie und Pädagogische Psychologie, 33, 91-102.

Schneider, W. (1985). Developmental trends in the metamemory - memory behavior relationship: An integrative review. In D. L. Forrest-Pressley, G. E. MacKinnon \& T. G. Waller (Eds.), Metacognition, cognition, and human performance (Vol. I, pp. 111-153). Orlando, FL: Academic Press.

Schneider, W. (1986). The role of conceptual knowledge and metamemory in the development of organizational processes in memory. Journal of Experimental Child Psychology, 42, 218-236.

Schneider, W. (1999). The development of metamemory knowledge in children. In. D. Gopher \& A. Koriat (Eds.), Attention and performance XVIII: Cognitive regulation of performance - interaction of theory and application (pp. 487-514). Cambridge, MA: MIT Press.

Schneider, W. (2000). Research on memory development: Historical trends and current themes. International Journal of Behavioral Development, 24, 407-420.

Schneider, W. \& Björklund, D. F. (1992). Expertise, aptitude, and strategic remembering. Child Development, 63, 461-473.

Schneider, W. \& Bjorklund, D. F. (1998). Memory. In W. Damon, D. Kuhn, \& R. S. Siegler (Eds.), Handbook of child psychology: Cognition, perception, and language (Vol. 2, pp. 467-521). New York: Wiley.

Schneider, W., Bjorklund, D. F. \& Maier-Brückner, W. (1996). The effects of expertise and IQ on children's memory: When knowledge ist and when it is not enough. International Journal of Behavioral Development, 19, 773-796

Schneider, W., Gruber, H., Gold, A. \& Opwis, K. (1993). Chess expertise and memory for chess positions in children and adults. Journal of Experimental Child Psychology, 56, 328-349.

Schneider, W., Körkel, J. \& Weinert, F. E. (1987). The effects of intelligence, self-concept, and attributional style on metamemory and memory behaviour. International Journal of Behavioral Development, 10, 281-299. 
Schneider, W., Körkel, J. \& Weinert, F. E. (1989). Domain-specific knowledge and memory performance: A comparison of high- and low-aptitude children. Journal of Educational Psychology, 81, 306-312.

Schneider, W. \& Pressley, M.(1997). Memory development between 2 and 20 ( $2^{\text {nd }}$ ed). Mahwah, NJ: Erlbaum.

Schneider, W., Schlagmuller, M. \& Visé, M. (1998). The impact of metamemory and domainspecific knowledge on memory performance. European Journal of Psychology of Education, 13, 91-103.

Schneider, W. \& Sodian, B. (1988). Metamemory-memory behavior relationships in young children: Evidence from a memory-for-location task. Journal of Experimental Child Psychology, 45, 209-233.

Schneider, W. \& Sodian, B. (1997). Memory strategy development: Lessons from longitudinal research. Developmental Review, 7, 442-461.

Schöne, C., Dickhäuser, O., Spinath, B. \& Stiensmeier-Pelster (2003). Das Fähigkeitsselbstkonzept und seine Erfassung. In J. Stiensmeier-Pelster \& F. Rheinberg (Hrsg.), Diagnostik von Selbstkonzept und Motivation und Selbstregulation [Tests und Trends, Jahrbuch der pädagogisch-psychologischen Diagnostik N.F., Band 2], (S. 3 - 14). Göttingen: Hogrefe.

Shallice, T. (1988). From neuropsychology to mental structure. Cambridge: Cambridge University Press.

Shallice, T. \& Warrington, E. K. (1970). Independent functioning of verbal memory stores: A neuropsychological study. Quarterly Journal of Experimental Psychology, 22, 261-273.

Shavelson, R. J., Hubner, J. J. \& Stanton, G. C. (1976). Self-Concept: Validation of construct interpretations. Review of Educational Research, 46 , 407 - 441.

Shimamura, A. P. (2000). Toward a cognitive neuroscience of metacognition. Consciousness and Cognition, 9, 313-323.

Siaw, S. N. \& Kee, D. W. (1987). Development of elaboration and organization in different socioeconomic-status and ethnic populations. In M. A. McDaniel \& M. Pressley (Eds.), Imagery and related mnemonic processes: Therories, individual differences, and applications. New York: Springer

Siegler, R. S. (1996). Emerging minds. The process of change in children's thinking. New York: Oxford University Press.

Siegler, R. S. (2001). Das Denken von Kindern. München: Oldenbourg

Siegler, R. S. \& Crowley, K. (1991). The microgenetic method: A direct means for studying cognitive deveopment. American Psychologist, 46, 606-620.

Siegler, R. S. \& Jenkins, E. A. (1989). How children discover new strategies. Hillsdale, NJ: Erlbaum. 
Siegler, R. S. \& Stern, E. (1998). A microgenetic analysis of conscious and unconscious strategy discoveries. Journal of Experimental Psychology: General, 127, 377-397.

Sigelman, C. (1999). Life-span human development. (3 ${ }^{\text {rd }}$ ed.). California: Brooks/Cole Publishing Co.

Smith, E. E. (1999). Working memory. In R. A. Wilson \& F. C. Keil (Eds.), The MIT encyclopedia of the cognitive sciences. Cambridge, MA: MIT Press.

Sodian, B. \& Schneider, W. (1999). Memory strategy development - Gradual increase, sudden insight or roller coaster? In F. E. Weinert \& W. Schneider (Eds.), Individual development from 3 to 12: Findings from the Munich Longitudinal Study (pp 61-77). Cambridge: Cambridge University Press.

Sommerville, S. C., Wellman, H. M. \& Cultice, J. C. (1983). Young children's deliberate reminding. The Jounral of Genetic Psychologie, 143, 87-96.

Srull, T. K. \& Weyer, R. S., Jr. (Eds.), (1993). The mental representation of trait and autobiographical knowledge about the self: Advances in social cognition. Hillsdale, NJ: Erlbaum.

Stemmler, M., Lösel, F. \& Erzigkeit, H. (1998). Analysis of longitudinal data: Introduction to special issue. Methods of Psychological Research Online, 3, 1-6.

Stiensmeier-Pelster, J., Balke, S. \& Schlangen, B. (1996). Lern- versus Leistungszielorientierung als Bedingungen des Lernfortschritts. Zeitschrift für Entwicklungspsychologie und Pädagogische Psychologie, 18, 169-187.

Taatgen, N. A. (1999). Learning without limits: From problem solving towards a unified theory of learning. PhD thesis, Artificial Intelligence, University of Groningen, Netherlands.

Tan, L. \& Ward, G. (2000). A recency-based account of the primacy effect in free recall. Journal of Experimental Psychology: Learning, Memory, and Cognition, 26, 15891625.

Tarkin, B. (1981). The effects of stimulus meaningfulness on children's spontaneous rehearsal strategies. Unpublished senior honors thesis, University of Massachusetts.

Tarkin, B., Myers, N. A. \& Ornstein, P. A. (1985). The effects of stimulus meaningfulness on children's spontaneous rehearsal strategies. Unpublished manuscript.

Tehan, G. \& Lalor, D. M. (2000). Individual differences in memory span: The contribution of rehearsal, access to lexical memory, and output speed. Quarterly Journal of Experimental Psychology: Human Experimental Psychology, 53A, 1012-1038.

Tewes, U., Schallberger, U. \& Rossmann, K. (2000). Hamburg-Wechsler-Intelligenztest für Kinder III (HAWIK III). Göttingen: Hogrefe. 
Tulving, E. (1982). Synergistic ecphory in recall and recognition. Canadian Journal of Psychology, 36, 130-147.

Tulving, E. (1983). Elements of episodic memory. Oxford: Oxford University Press.

Turner, J. E., Henry, L. A. \& Smith, P. T. (2000). The development of the use of long-term knowledge to assist short-term recall. Quarterly Journal of Experimental Psychology, $53,457-478$.

Vollmeyer, R. \& Rheinberg, F. (1998). Motivationale Einflüsse auf Erwerb und Anwendung von Wissen in einem computersimulierten System. Zeitschrift für Pädagogische Psychologie, 12, 11-23.

Ward, G. (2001). A critique of the working memory model. In J. Andrade (Ed.), Working memory in perspective (pp. 219-239). Hove: Psychology Press.

Ward, G. (2002). A recency-based account of the list length effect in free recall. Memory \& Cognition, 30, 885-892.

Waters, H. S. (2000). Memory strategy development: Do we need yet another deficiency? Child Development, 71, 1004-1012.

Waters, H. S. \& Andreassen, C. (1983). Children's use of memory strategies under instruction. In M. Presseley \& J. R. Levin (Eds.), Cognitive strategies: Developmental, educational, and treatment-related issues. New York: Springer.

Weinert, F.E. \& Schneider, W. (1996). Entwicklung des Gedächtnisses. In D. Albert \& K.H. Stapf (Hrsg.) Enzyklopädie der Psychologie. Themenbereich C: Theorie und Forschung. Serie II: Kognition, Band 4: Gedächtnis (S. 433-487). Göttingen: Hogrefe.

Weinert, F. E. \& Schneider, W. (Eds.), (1999). Individual development from 3 to 12: Findings from the Munich longitudinal study. Cambridge: University Press.

Weissberg, J. A. \& Paris, S. G. (1986). Young children's remembering in different contexts: A reinterpretation of Istomina's study. Child Development, 57, 1123-1129.

Wohlwill, J. F. (1973). The study of behavioral development. New York: Academic Press.

Yussen, S. R., Gagne, E., Gargiulo, R. \& Kunen, S. (1974). The distinction between perceiving and memorizing in elementary school children. Child Development, 45, 547-551.

Zanobini, M. \& Usai, C. (2002). Domain-specific self-concept and achievement motivation in the transition from primary to low middle school. Educational Psychology, 22, 203-217. 


\section{Anhang A}

Untersuchungsmaterial: Wortlisten für die Gedächtnis-Aufgabe

Die in Klammern stehenden Wörter wurden nur beim ersten Messzeitpunkt eingesetzt.

Übungsliste 1

1.

2.

3.

4.

5.

6.

1.

2.

3.

4.

5.

6.

7.

8.

9.

10.

11.

12.

13.

14.
Lernliste 1:

Radio

Orgel

Schaukel

Krokus

Blitz

Ahorn

Reifen

Buch

Gabel

Maurer

Klavier

Teddy

Pinsel

Hantel

Regen

Birke

Ampel

See

(Nelke)

(Stroh)

\section{Übungsliste 2}

(Telefon)

(Harfe)

(Kreisel)

(Efeu)

(Schnee)

(Palme)
Lernliste 2:

Hupe

Sonne

Brief

Teller

Maler

Geige

Puzzle

Tanne

Füller

Angel

Rose

Schild

(Wiese)

(Ziegel)
Lernliste 3:

Auspuff

Eiche

Zeitung

Topf

Lehrer

Berg

Flöte

Puppe

Kreide

Barren

Tulpe

Schranke

(Wolken)

(Stein) 


\section{Anhang B}

Untersuchungsmaterial: Nachbefragung Gedächtnis-Aufgabe.

Erfassung der metamemorialen Bewusstheit für Rehearsal

„Das hast du ja ganz toll gemacht. Kannst du mir sagen, wie du dir DAS ALLES merken konntest?! Wie hast du das denn gemacht? Hattest du irgendeinen Kniff dabei?““

„Und was ist so gut daran, dir die Wörter so zu merken, dass du dir . . .

Für einmalige Nennung: jedes Wort noch einmal sagst?

Für sing. Rehearsal: jedes Wort mehrfach wiederholst?

Für kum. Rehearsal: alle bislang gehörten Wörter wiederholst?

Sonstiges: Jeweiliges Lernverhalten wiedergeben 


\section{Anhang C}

Untersuchungsmaterial: Zahlennachsprechen vorwärts

\begin{tabular}{c|l}
$\begin{array}{c}\text { Gruppe } \\
\text { 1) }\end{array}$ & $2-9$ \\
& $4-6$ \\
\hline 2) & $3-8-6$ \\
& $6-1-2$ \\
\hline 3) & $3-4-1-0$ \\
& $6-1-5-8$ \\
\hline 4) & $8-4-2-3-9$ \\
\hline 5) & $5-2-1-8-6$ \\
\hline 6) & $3-8-9-1-0-4$ \\
& $0-9-6-4-8-3$ \\
\hline 7) & $9-8-5-2-1-6-3$ \\
\hline 8) & $2-6-4-5-9-0-6-3$ \\
& $5-3-8-0-1-2-4-6-9$ \\
\hline
\end{tabular}

Untersuchungsmaterial: Zahlennachsprechen rückwärts

\begin{tabular}{c|l}
$\begin{array}{c}\text { Gruppe } \\
\text { 1) }\end{array}$ & $2-5$ \\
& $6-3$ \\
\hline 2) & $5-0-4$ \\
& $2-5-9$ \\
\hline 3) & $0-2-9-6$ \\
& $8-4-9-3$ \\
\hline 4) & $4-1-3-5-0$ \\
& $9-0-8-5-2$ \\
\hline 5) & $1-6-5-2-9-8$ \\
& $3-6-0-1-9-4$ \\
\hline 6) & $8-5-9-2-3-4-2$ \\
& $4-5-0-9-2-8-1$ \\
\hline 7) & $6-9-1-6-3-2-5-8$ \\
& $3-1-0-9-5-4-8-1$
\end{tabular}




\section{Anhang D}

Untersuchungsmaterial: Items und Instruktionen zur Erfassung des strategiespezifischen Metagedächtnisses für Rehearsal

1. Ihr seid bei eueren Nachbarn zum Dia-Abend über eine Berlinfahrt eingeladen. Deine Mutter kann leider nicht mit und bittet dich, ihr später zu erzählen, welche Berliner Sehenswürdigkeiten auf den Bildern zu sehen waren. Wie merkst du dir die Sehenswürdigkeiten?

$\theta \quad$ Du sagst bei jedem Bild einmal, was für eine Sehenswürdigkeit zu sehen ist.

Beispiel: „Brandenburger Tor“ und dann so weiter.

$\theta \quad$ Du wiederholst für dich nach jedem Bild immer wieder alle Sehenswürdigkeiten, die du bisher gesehen hast.

Beispiel: „Brandenburger Tor, Wannsee, Bundestag“ - „Brandenburger Tor, Wannsee, Bundestag“

$\theta \quad$ Du sagst dir nach jedem Bild leise dreimal vor, was du auf diesem Bild gesehen hast. Beispiel: „Brandenburger Tor, Brandenburger Tor, Brandenburger Tor“

Nun darfst du wieder Noten vergeben: 1, 2, 3, 4 oder 5. Schreibe die Noten bitte in die Kästchen davor. Du kannst eine Note auch mehrmals vergeben.

\section{$\theta$ zusätzliche Hilfestellung erforderlich (z.B. Wiederholung mit eigenen Worten)}

Art der Hilfestellung:

2. Du machst mit deiner Klasse einen Ausflug in den Zoo. Später willst du deinen Eltern erzählen, welche Tiere du dort gesehen hast. Was machst du?

$\theta \quad$ Du sagst dir während des Ausflugs immer wieder vor, welche Tiere du gesehen hast. (Beispiel: „Löwe, Schlange, Adler“ - „Löwe, Schlange, Adler“)

$\theta \quad$ Bei jedem Tier, an dem du stehen bleibst, sagst du dir den Namen ein paar Mal vor. (Beispiel: „Löwe, Löwe, Löwe“)

$\theta \quad$ Du überlegst dir nach dem Ausflug, was dir am besten und was dir am schlechtesten gefallen hat. (Beispiel: „Der Löwe war am schönsten“).

Nun darfst du wieder Noten vergeben: 1, 2, 3, 4 oder 5. Schreibe die Noten bitte vorne in die Kästchen. Du kannst eine Note auch mehrmals vergeben.

\section{$\theta$ zusätzliche Hilfestellung erforderlich (z.B. Wiederholung mit eigenen Worten)} Art der Hilfestellung: 
3. Du hörst mit deiner Freundin eine Hörspielkassette. Es geht um zwei Kinder, die sich auf eine weite Reise begeben. Ihr wollt herausfinden, wer sich am Schluss die meisten Orte merken kann, an denen die Kinder gewesen sind. Der Gewinner soll einen tollen Preis bekommen. Wie wirst du dir die Orte merken?

$\theta \quad$ Du wiederholst jeden einzelnen Ortsnamen mehrere Male.

Beispiel: „Paris, Paris, Paris“

$\theta \quad$ Du sagst dir eine ganze Reihe von Ortsnamen mehrfach vor.

Beispiel: „Paris, London, Rom“ - „Paris, London, Rom“

$\theta \quad$ Du überlegst dir, welche Orte du davon bereits kennst.

Beispiel: „In Paris haben wir schon einmal Urlaub gemacht“

Nun darfst du wieder Noten vergeben: 1, 2, 3, 4 oder 5. Schreibe die Noten bitte vorne in die Kästchen. Du kannst eine Note auch mehrmals vergeben.

$\theta$ zusätzliche Hilfestellung erforderlich (z.B. Wiederholung mit eigenen Worten)

Art der Hilfestellung: 